\title{
SELECTION OF PROCESS CONTROL STRUCTURE BASED ON ECONOMICS
}

\author{
by \\ Lawrence Trevelyan NARRAWAY, B.E. \\ October 1992
}

A thesis submitted for the degree of Doctor of Philosophy of the University of London and for the Diploma of Membership of the Imperial College

Department of Chemical Engineering and Chemical Technology

Imperial College of Science, Technology and Medicine

London SW7 


\begin{abstract}
It has been recognised that the effect of dynamics on process economics is heavily dependent on the implemented controller, and that the physically attainable controller performance is limited by the choice of controlled and manipulated variables. This work considers the systematic selection of economically optimal square regulatory control structures from the set of all possible measurements and manipulated variables. This is a combinatorial problem, as typical processes have millions of potential control structures, where a control structure consists of a set of variables to be controlled and manipulated, but does not include a control law relating the variables.
\end{abstract}

The solution of such a synthesis problem requires an analysis method. Historical approaches to control structure evaluation consider "controllability indices", and their trade off against steady state economics, whereas the economic indicators described in this thesis allow direct comparison and trade off of control structures against steady state economics.

Assuming a method for assessing control structure economics, a hierarchical decomposition of the design problem into steady state and dynamic problems is demonstrated.

A method for assessing the effect of dynamics on process economics for a specific control structure without tuning a controller is developed, based on linearised process models, linear systems theory and constraint control concepts. This assessment is extended to consider the control structure selection problem, resulting in a mixed integer linear programming (MILP) outer approximation to a mixed integer nonlinear programming (MINLP) problem.

A mixed integer nonlinear optimal control problem is posed for the problem of selecting an optimal multiloop proportional-integral process control structure.

The above problems are formulated as standard optimisation problems, and solved by appropriate techniques. Experience with the methods is presented as a set of case studies.

Finally the novel features and applications of the work are discussed along with potential areas of further research. 


\section{Acknowledgements}

I want to express my gratitude to my supervisor, Prof. J.D. Perkins, for his invaluable guidance, advice and support during the course of this work.

I would like to thank my colleagues in the Centre for Process Systems Engineering for their friendship.

Finally, I would also like to thank the British Council and the Association of Commonwealth Universities for funding this work. 


\section{Contents}

$\begin{array}{ll}\text { List of Figures } & 7\end{array}$

$\begin{array}{lc}\text { List of Tables } & 8\end{array}$

$\begin{array}{ll}\text { List of symbols } & 10\end{array}$

1 Introduction $\quad 19$

1.1 Process control objectives . . . . . . . . . . . . . . . 22

1.2 Control structure selection and ranking . . . . . . . . . . . 27

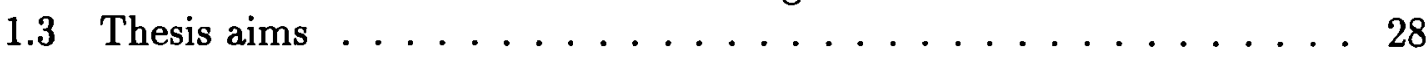

1.4 Thesis outline ....................... 29

2 Dynamic economics in process design 31

2.1 The process synthesis problem . . . . . . . . . . . . . 31

2.2 The combined design problem ... . . . . . . . . . . . 32

2.3 Steady state bounding of dynamic economics . . . . . . . . . . 36

2.4 Systematic steady state design procedures . . . . . . . . . . . . 39

2.5 Decomposition based combined design procedures . . . . . . . . . . 41

2.5.1 Algorithm 1: Optimal steady state/dynamic flowsheet . . . . . 42

2.5.2 Algorithm 2: Optimal open loop design set . . . . . . . . . 43

2.5 .3 Example .................... . . 44

2.6 Summary ............................. 45

3 Historical review $\quad \mathbf{4 7}$

3.1 Control system synthesis . . . . . . . . . . . . . . . . . 47

3.1.1 Steady state control structure selection . . . . . . . . . 48

3.1.2 Multilevel synthesis . . . . . . . . . . . . 50

3.1.3 Cause and effect digraphs .............. . . 53

3.1.4 A unified, systematic approach ............. 57

3.1.5 Other structural approaches .............. 63

3.1.6 Robustness based structure selection . . . . . . . . . . . 64

3.1.7 Summary of control system synthesis techniques . . . . . . . 64

3.2 Controllability analysis ................. 65

3.3 Economic analysis of dynamics ................ 69

3.4 Linear versus nonlinear dynamic analysis . . . . . . . . . . 73

3.5 Summary . . . . . . . . . . . . . . . . . 74

4 Linear analysis of dynamic economics $\quad 76$

4.1 Linear dynamic economics . . . . . . . . . . . . . . 76

4.2 Case study - Froth flotation circuits . . . . . . . . . . 80

4.3 Summary ......................... 90 
5 Control structure selection based on linear analysis 91

5.1 General linear dynamic economic analysis . . . . . . . . . . . 91

5.1 .1 Calculation of $\tilde{\sigma}$ and $U \ldots \ldots$. . . . . . . . . . . . . . . . . . . . .

5.2 MILP control structure selection . . . . . . . . . . . . 98

5.2.1 Perfect control specifications . . . . . . . . . . . 100

5.2 .2 Linear lower bound on $\tilde{\rho} \ldots \ldots . \ldots 101$

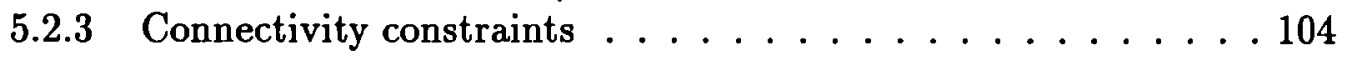

5.2.4 Perfect control problem ................ . . 111

5.2.5 MILP control structure selection algorithm . . . . . . . . . 112

5.2 .6 Implementation . . . . . . . . . . . . . . 113

5.3 Summary . . . . . . . . . . . . . . . . . . . 114

6 MILP case studies $\quad \mathbf{1 1 5}$

6.1 Double effect evaporator case study . . . . . . . . . . . . . 115

6.1.1 MILP selection of optimal control structure . . . . . . . 118

6.1.2 Case 1 - Base case disturbance . . . . . . . . . . . 122

6.1.3 Case 2 - Reduced steam variability .......... . 125

6.1.4 Case 3 - Multiple disturbance frequencies . . . . . . . . 126

6.1 .5 Computational results . . . . . . . . . . . . . 129

6.1 .6 Section summary . . . . . . . . . . . . . . . . . . . . . . . . . . . . . . . . . .

6.2 Mount Isa Mines froth flotation circuit . . . . . . . . . . . 130

6.2.1 Case 1 - Optimistic sensor/actuator costs . . . . . . . 138

6.2.2 Case 2 - Pessimistic sensor/actuator costs . . . . . . . 143

6.2.3 Computational results . . . . . . . . . . . . 146

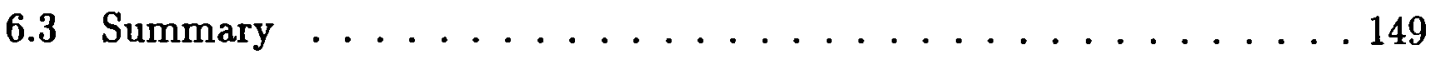

7 Nonlinear control structure selection $\quad \mathbf{1 5 1}$

7.1 Formulation of PI controllers . . . . . . . . . . . . . . 154

7.1.1 Full superstructure formulation . . . . . . . . . . 157

7.1.2 Shifted superstructure formulation . . . . . . . . . 158

7.2 Multiloop structure selection problem summary . . . . . . . . . 160

7.3 Dynamic optimisation . . . . . . . . . . . . . . . 162

7.4 MINLP Solution Methods . . . . . . . . . . . . . . . . . . 164

7.4.1 Explicit enumeration . . . . . . . . . . . . 165

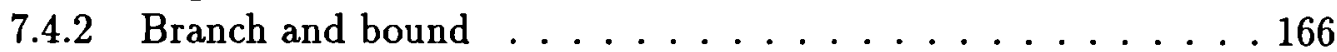

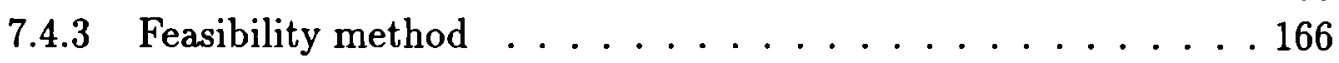

7.4.4 Outer Approximation methods ............ . . . . . . . . . . . . . . . .

7.5 Implementation . . . . . . . . . . . . . . . . 172

8 MIDAOPT case studies $\quad \mathbf{1 7 3}$

8.1 Chan et al flotation circuits . . . . . . . . . . . . . 173

8.2 Double effect evaporator . . . . . . . . . . . . . 176

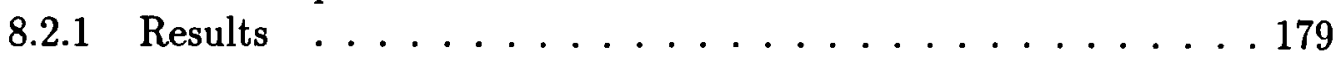

8.2.2 Full superstructure results . . . . . . . . . . . . . 184

8.2.3 Full superstructure case - reduced weights . . . . . . 187

8.3 Summary and conclusions . . . . . . . . . . . . . . . 189 
9 Summary and conclusions $\quad \mathbf{1 9 0}$

9.1 Linear dynamic economics . . . . . . . . . . . . . . . . . 191

9.2 MILP control structure selection . . . . . . . . . . . . . . . . 193

9.3 MIDAOPT control structure selection . . . . . . . . . . . . . 194

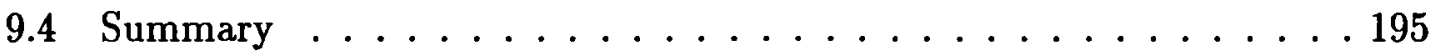

$\begin{array}{ll}\text { References } & 196\end{array}$

A Froth flotation circuit model $\quad 206$

A.1 Cell model . . . . . . . . . . . . . . . . . . . . 206

A.2 Operating conditions and constraints ............ 207

$\begin{array}{lr}\text { B Scaling of condition numbers } & 209\end{array}$

B.1 Minimum condition number of a triangular matrix . . . . . . . 210

C Tight MILP formulation $\quad \mathbf{2 1 1}$

C.1 Improved linear lower bound on complex magnitudes . . . . . . . 211

C.1.1 Proof of bounding properties . . . . . . . . . . . . 212

C.1.2 Maximum relative error . . . . . . . . . . . . 213

C.2 Improved lower bound for $\tilde{s} \ldots \ldots 213$

D Double effect evaporator models and process items $\quad 216$

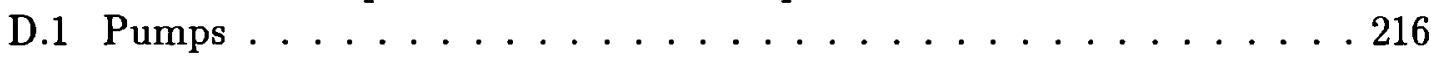

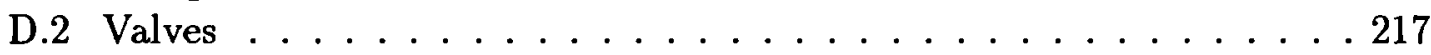

D.3 Evaporator . . . . . . . . . . . . . . . 217

D.3.1 Evaporator design . . . . . . . . . . . . . . 217

D.3.2 Evaporator model . . . . . . . . . . . . . . . . 217

D.4 Condition number plots . . . . . . . . . . . . . 220

E Mount Isa Mines model changes $\quad 225$

E.1 Flotation cell model . . . . . . . . . . . . . . . . . . . . . . . . . . . . . . . . . . . .

E.2 Collector addition model . . . . . . . . . . . . . 226

E.3 Summary of steady state operating conditions . . . . . . . . . 226

F Properties of $\gamma_{\infty}^{*}$ for square block triangular transfer functions $\quad \mathbf{2 3 0}$

G Path constraints in dynamic optimisation 232

G.1 Scaling of path constraints . . . . . . . . . . . . . . 232

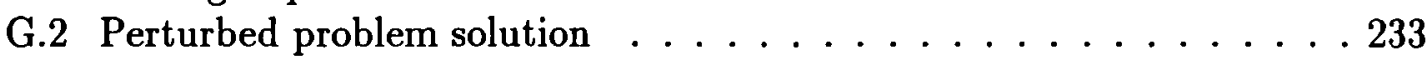




\section{List of Figures}

2.1 Geometric interpretation of the combined design problem . . . . . 36

3.1 Hierarchical control system synthesis structure . . . . . . . . . 52

3.2 Liquid-liquid heat exchanger and associated digraph . . . . . . . 54

3.3 Sensed variable tree .................. 56

4.1 Froth flotation cell . . . . . . . . . . . . . . . 82

4.2 Froth flotation circuit flowsheets . . . . . . . . . . . 83

4.3 Process recoveries with no control . . . . . . . . . . . 86

4.4 Perfect control recoveries (recovery control) . . . . . . . . . . . 86

4.5 Process recoveries with BLT constraint control . . . . . . . . . . 87

$4.6 l_{\infty}$ minimum condition number (recovery control) $\ldots \ldots \ldots 7$

6.1 Double effect evaporator flowsheet . . . . . . . . . . . . 116

$6.2 l_{\infty}$ minimum condition number plot for disturbance case $1 \ldots 124$

6.3 Mount Isa Mines froth flotation circuit . . . . . . . . . . . . 132

$6.4 l_{\infty}$ minimum condition number plot for MIM case $1 \ldots 140$

7.1 Full PI controller superstructure . . . . . . . . . . . . . 158

7.2 Shifted controller superstructure f. . . . . . . . . . . . 160

8.1 Nonconvex constraints in MINLP problems . . . . . . . . . . 184

D.1 Evaporator geometry . . . . . . . . . . . . . . 218

D.2 $l_{\infty}$ minimum condition number plot for case $2 \ldots \ldots . \ldots \ldots$

D.3 $l_{\infty}$ minimum condition number plot for multiple frequency case . . . 224 


\section{List of Tables}

2.1 Design decomposition example ... . . . . . . . . . . . 45

3.1 Heat exchanger structural array . . . . . . . . . . . . 55

4.1 Results of steady-state optimisation of flotation circuits . . . . . 85

4.2 Average recovery for flotation circuits at disturbance frequency of $1 / 30$ cycles $/ \min \ldots \ldots \ldots . \ldots . \ldots 88$

$4.3 l_{\infty}$ minimum condition number at $w=1 / 30$ cycles $/ \mathrm{min} \ldots \ldots 8$

6.1 Nominal evaporator operating conditions f . . . . . . . . . 118

6.2 Optimum steady state variable summary . . . . . . . . . . . . . 119

6.3 Steady state active constraint summary . . . . . . . . . . . . 120

6.4 Potential measured variables on the double effect evaporator . . . . 120

6.5 Measurement/valve costs for double effect evaporator . . . . . . . . . 121

6.6 Base case disturbance at $\omega=0.005$ cycles $/ \mathrm{min} \ldots \ldots . . \ldots 122$

6.7 Optimal control structures for disturbance case $1 \ldots \ldots 122$

6.8 Case 2 disturbance at $\omega=0.005$ cycles $/ \mathrm{min} \ldots \ldots . . \ldots 125$

6.9 Optimal control structures for disturbance case $2 \ldots \ldots 125$

6.10 Summary of evaporator disturbances . . . . . . . . . . . . 126

6.11 Optimal control structures for multiple disturbance frequencies . . . 127

6.12 Cost comparison of dual and composition control . . . . . . . . 128

6.13 Nominal operating conditions for MIM circuit . . . . . . . . . . 135

6.14 Optimal steady state parameters of MIM circuit . . . . . . . . 136

6.15 MIM steady state constraint summary . . . . . . . . . . . . 137

6.16 Disturbance magnitudes for MIM case study . . . . . . . . . . . . 137

6.17 Potential measured and manipulated variables on the MIM circuit $\quad 138$

6.18 Optimistic instrumentation costs for MIM circuit . . . . . . . . . 139

6.19 Optimal control structure for optimistic costs . . . . . . . . . . 139

6.20 Pessimistic instrumentation costs for MIM circuit . . . . . . . . . . 144

6.21 Optimal control structure for pessimistic costs . . . . . . . . . . 145

6.22 Major iterations to solution and prove optimality: case $1 \ldots 147$

6.23 Major iterations to solution and prove optimality: case $2 \ldots 147$

8.1 Possible flotation circuit control loops . . . . . . . . . . . . . 173

8.2 MIDAOPT results for flotation circuit $1 \ldots \ldots$. . . . . . . . 175

8.3 MIDAOPT results for flotation circuit $8 \ldots \ldots 176$

8.4 Initial structures for evaporator MIDAOPT case study . . . . . . . 179

8.5 Shifted superstructure evaporator results . . . . . . . . . . . . . 181

8.6 Full superstructure evaporator results . . . . . . . . . . . . 186

8.7 Full superstructure results - reduced weights $\ldots \ldots 188$

A.1 Steady state operating conditions . . . . . . . . . . . 207 


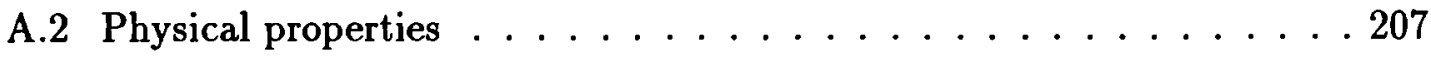

C.1 Maximum relative error for improved complex magnitude bound _ . 213

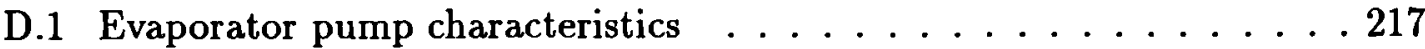

D.2 Symbol definitions for evaporator model . . . . . . . . . 221

D.3 Evaporator parameters . . . . . . . . . . . . 222 


\section{List of symbols}

\section{Chapter 1}

$h$

$h^{\prime}$

$h^{\prime \prime}$

$J$

$p$

$p_{0}$

$t$

$y$

subscript 0

superscript *

\section{Chapter 2}

c

$D$

$d$

$f$

$g^{\prime}$

$g$

$h$

$I$

$J$

$J_{d y n}^{*}$

$J_{s s}^{*}$

$J_{d y n}^{I}$

$J_{\text {ol }}^{I}$

$J_{s s}^{I}$

$J_{d y n}^{\Theta}$

$J_{o l}^{\Theta}$

$k$ process constraints

active process constraints

inactive process constraints

objective function

process disturbances

steady state (nominal) disturbances

time

process variables

steady state operating point

optimal operating point

controller equations

steady state constraints

design parameters

state equations

algebraic equations

augmented algebraic equations

process constraints

current flowsheet in design procedure

objective function

optimum dynamic design objective function

optimum steady state design objective function

optimum dynamic objective function of flowsheet $I$

optimum open loop objective function of flowsheet $I$

optimum steady state objective function of flowsheet $I$

optimum dynamic objective function of flowsheet $\Theta$

optimum open loop objective function of flowsheet $\Theta$

controller parameters 


$\begin{array}{ll}n_{r} & \text { dimension of vector } r \\ P(t) & \text { set of all possible process disturbances } \\ p & \text { disturbance parameters } \\ p_{0} & \text { nominal disturbance parameters } \\ \tilde{p} & \text { dynamic deviation disturbances } \\ t & \text { time } \\ u & \text { manipulated variables } \\ X & \text { integer variables } \\ x & \text { state variables } \\ \dot{x} & \text { time derivatives of } x \\ z & \text { algebraic variables } \\ \text { superscript high } & \text { simple upper bound } \\ \text { superscript low } & \text { simple lower bound } \\ \text { subscript } 0 & \text { steady state (nominal) value } \\ \Lambda & \text { open loop design set } \\ \Theta & \text { the best flowsheet located in the design procedure }\end{array}$

\section{Chapter 3}

$\begin{array}{ll}A-F & \text { (structural) state space matrices } \\ C_{n j} & j \text { th alternative multiloop controller for process unit } n \\ C_{r, n j} & \text { coordinated revision of } C_{n j} \\ c_{n i} & \text { ith potential SISO control loop for process unit } n \\ d & \text { process parameters } \\ G, G(s) & \text { process transfer function } \\ \tilde{G} & \text { model of the process transfer function } \\ G_{c} & \text { controller transfer function } \\ G_{p}(s) & \text { disturbance transfer function } \\ \tilde{G}_{-} & \text {minimum phase component of } \tilde{G} \\ \tilde{G}_{+} & \text {non-minimum phase component of } \tilde{G} \\ h & \text { process constraints } \\ J & \text { linear estimate of the dynamic economics } \\ J_{0} & \text { steady state optimal nonlinear economics }\end{array}$




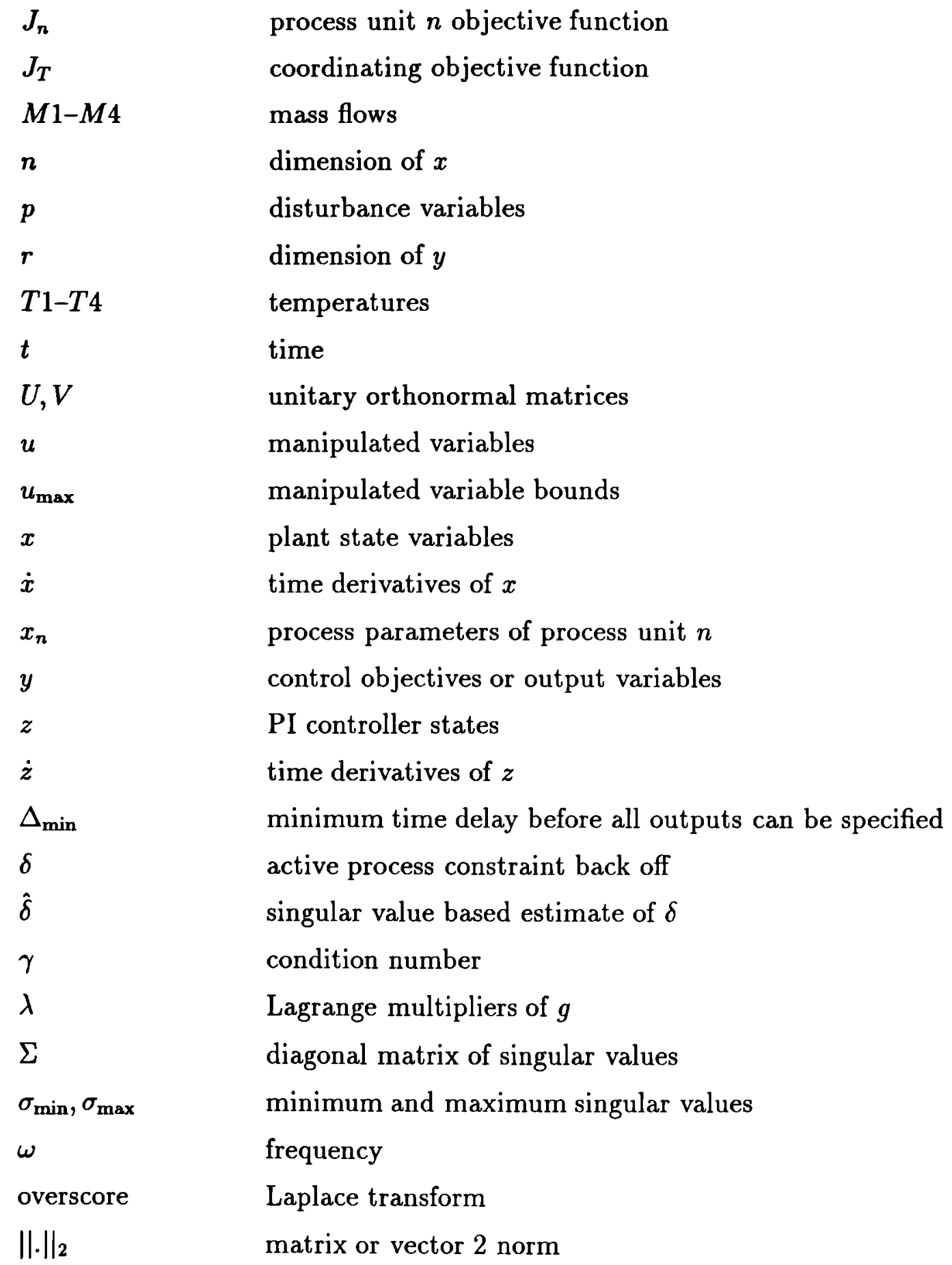

\section{Chapter 4}
A-H
state space matrices
$G(s), G(j w)$
transfer function matrix
I
identity matrix 


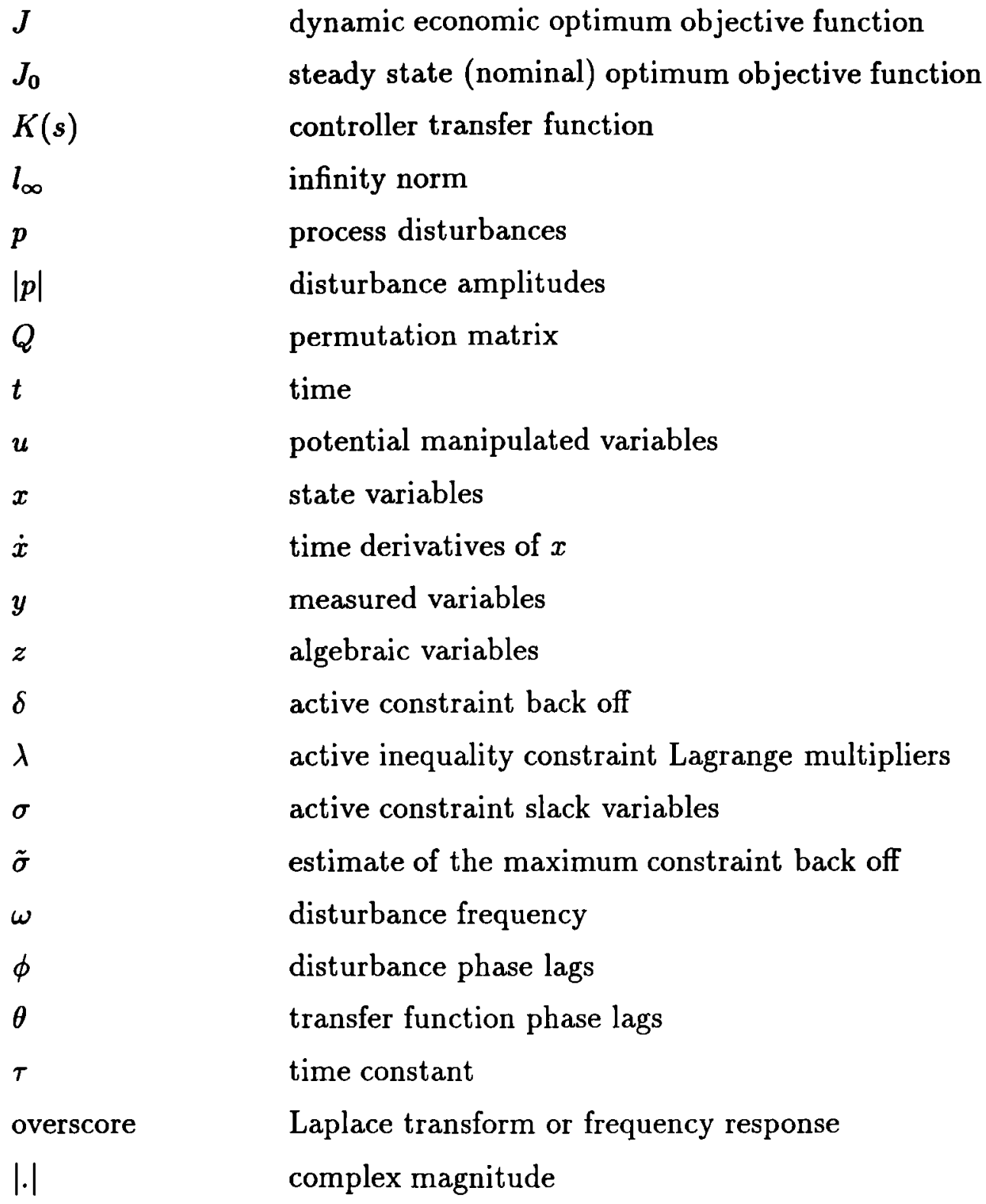

\section{Chapter 5}

$\begin{array}{ll}A-H, K-P, R & \text { state space matrices } \\ a_{i j} & \text { structural transfer function element } \\ a, b & \text { vector or scalar constants } \\ d & \text { design parameters } \\ f & \text { state equations } \\ g & \text { algebraic equations } \\ G_{c}(s) & \text { process controller transfer function } \\ h & \text { inequality constraints }\end{array}$




\begin{tabular}{|c|c|}
\hline$I$ & identity matrix \\
\hline$J$ & objective function \\
\hline$J_{M}$ & MILP objective function \\
\hline$J_{p c}$ & perfect control problem objective function \\
\hline$n_{r}$ & dimension of vector $r$ \\
\hline$P(\omega)$ & set of possible disturbances \\
\hline$p$ & disturbance variables \\
\hline$p(\omega)$ & disturbance amplitude at frequency $\omega$ \\
\hline$p_{\max }(\omega)$ & maximum disturbance amplitude at frequency $\omega$ \\
\hline$Q_{\mathbf{u}}, Q_{\mathbf{z}}$ & permutation matrices \\
\hline$q, \eta$ & general LP variables \\
\hline$U$ & estimate of the maximum dynamic variation of $u$ \\
\hline$u$ & potential manipulated variables \\
\hline$u_{\max }(\omega)$ & estimate of maximum constraint back off at frequency $\omega$ \\
\hline$\tilde{u}$ & linear approximation to $U$ \\
\hline$X, \mathcal{X}, Z$ & integer variables \\
\hline$x$ & state variables \\
\hline$\dot{x}$ & time derivatives of $x$ \\
\hline$z$ & potential measured variables \\
\hline$\alpha, \beta, \gamma, \delta$ & cost coefficients \\
\hline$\Delta(s)$ & transfer function between $\sigma$ and $p$ \\
\hline$\epsilon, \varepsilon$ & sensor/actuator costs \\
\hline$\Gamma, \Lambda$ & matrices \\
\hline$\Phi$ & disturbance phase lags \\
\hline$\Sigma$ & absolute slack variables \\
\hline$\sigma$ & deviation slack variables \\
\hline$\tilde{\sigma}$ & estimate of the maximum constraint back off \\
\hline$\sigma_{\max }(\omega)$ & estimate of maximum constraint back off at frequency $\omega$ \\
\hline$\tilde{\rho}$ & linear approximation to $\tilde{\sigma}$ \\
\hline$\omega$ & disturbance frequency \\
\hline$\omega_{d}$ & disturbance frequency range \\
\hline subscript 0 & steady state (nominal) value \\
\hline subscript $c$ & complex part \\
\hline
\end{tabular}




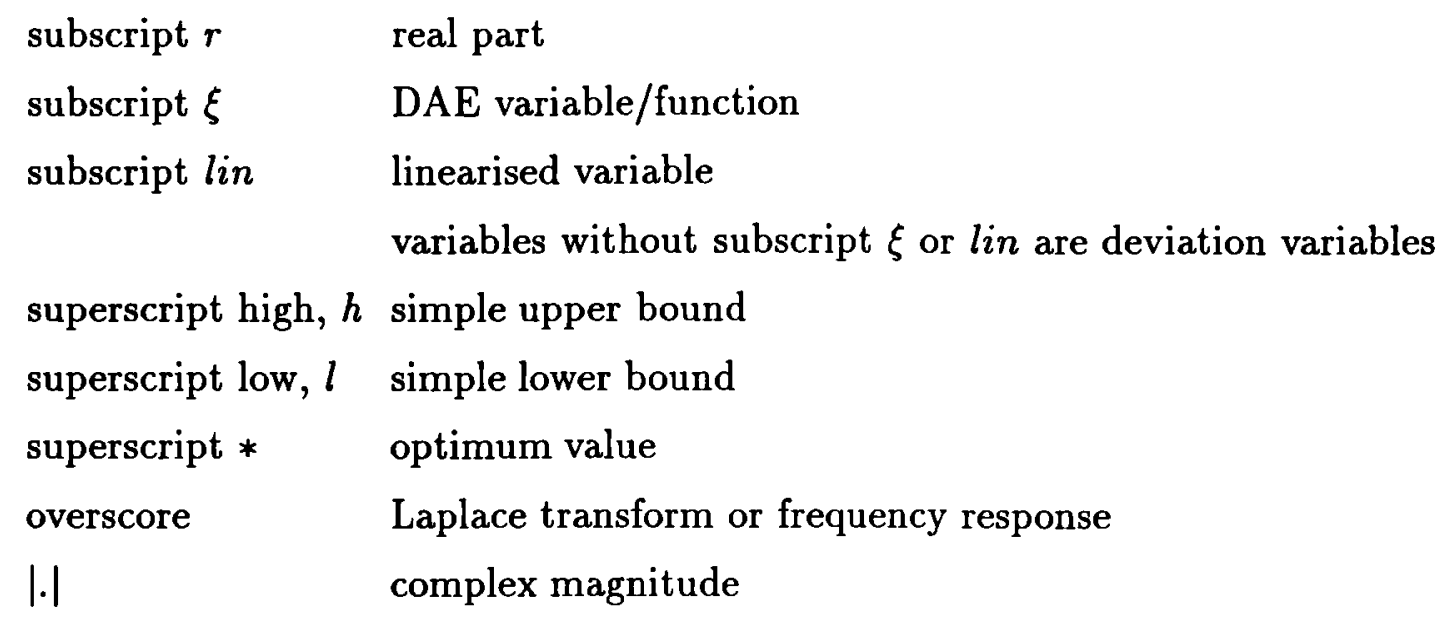

\section{Chapter 6}

$\begin{array}{ll}A, B, Q, R & \text { matrices } \\ A_{T} & \text { tailings assay } \\ a & \text { aeration rate } \\ C & \text { concentrate flow } \\ C_{A} & \text { annualised instrumentation cost } \\ C_{A}^{\prime} & \text { annualised instrumentation cost including maintenance } \\ C_{I} & \text { installed instrumentation cost } \\ C_{I}^{\prime} & \text { installed instrumentation cost including maintenance } \\ C_{v} & \text { valve coefficient } \\ F_{A} & \text { production of } A \\ F_{k} & \text { flow through pump } k, k=f, i, p \\ F_{s} & \text { product solute flow } \\ F_{s t e a m} & \text { steam flow, kg/min } \\ F_{t} & \text { total product flow } \\ F_{w} & \text { product water flow } \\ g_{i j} & \text { transfer function element } \\ i & \text { interest rate } \\ K_{i} & \text { flotation rate coefficient } \\ K_{v} & \text { valve coefficient } \\ L V 1-L V 3 & \text { liquid flow valve identifiers } \\ l_{\infty} & \text { infinity norm } \\ M F & \text { maintenance factor } \\ & \end{array}$




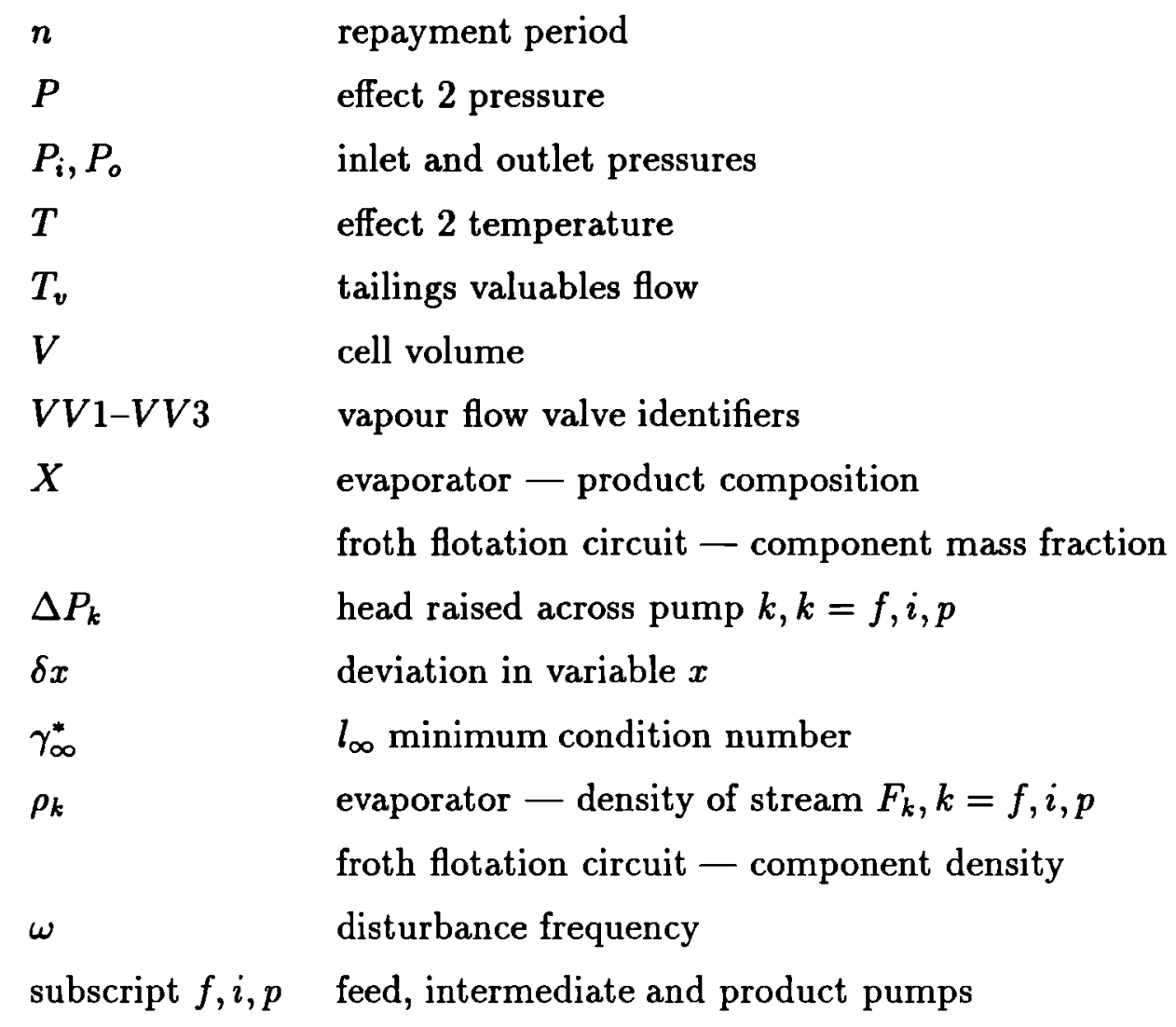

\section{Chapter 7}

$\begin{array}{ll}A, B, C & \text { matrices } \\ a, b, \alpha & \text { constants } \\ B^{\pi} & \text { set of indices of vector } X^{\pi} \text { with unity elements } \\ c & \text { controller equations } \\ D & \text { steady state constraints } \\ d & \text { design variables } \\ e & \text { controller errors } \\ f & \text { state equations } \\ g & \text { algebraic equations } \\ h & \text { process constraints } \\ I & \text { controller integral actions } \\ \dot{I} & \text { time derivative of } I \\ J & \text { objective function } \\ \mathcal{J} & \text { nonlinear part of objective function } \\ J_{A} & \text { master problem objective function }\end{array}$




\begin{tabular}{|c|c|}
\hline$K$ & controller proportional gains \\
\hline$k$ & controller parameters \\
\hline$L, M$ & discrete dynamic optimisation constraints \\
\hline$m$ & measured variables \\
\hline$N$ & terminal path constraints \\
\hline$N^{\pi}$ & set of indices of vector $X^{\pi}$ with zero elements \\
\hline$n_{x}$ & dimension of vector $x$ \\
\hline$P(t)$ & the set of all possible disturbances \\
\hline$p$ & disturbance variables \\
\hline$\tilde{p}$ & dynamic deviation disturbances \\
\hline$p^{\pi}, q^{\pi}, r, s_{0}^{\pi}$ & constraint violation slack variables \\
\hline$s$ & controller set points \\
\hline$\dot{s}$ & time derivatives of $s$ \\
\hline$T$ & controller parameters \\
\hline$t$ & time \\
\hline$t_{f}$ & finite time horizon \\
\hline$u$ & manipulated variables \\
\hline$w, \rho$ & penalty weights \\
\hline $\mathcal{X}$ & pseudo-integer variables \\
\hline$X$ & integer variables \\
\hline$x$ & state variables \\
\hline$\dot{x}$ & time derivatives of $x$ \\
\hline$z$ & algebraic variables \\
\hline$\Delta u$ & controller actions \\
\hline$\epsilon$ & control loop costs \\
\hline$\Gamma$ & equality constraint relaxation direction matrix \\
\hline$\lambda$ & equality constraint Lagrange multipliers \\
\hline$\mu$ & nominal valve positions \\
\hline$\nu$ & linear approximation to $\mathcal{J}$ \\
\hline$\Pi$ & current iteration \\
\hline$\pi$ & iteration counter \\
\hline$\Phi$ & a subset of the range $0 \leq t \leq t_{f}$ \\
\hline$d$ & elements of $\Phi$ \\
\hline
\end{tabular}


$\tau$

$\theta$

superscript high

superscript low

subscript 0

\section{Chapter 8}

e

$F_{s}$

$F_{t}$

$F_{w}$

I

$\dot{I}$

K

$\mathcal{K}$

$L V 1-L V 3$

$m$

$P$

$P_{c}$

$s$

$T$

$\mathcal{T}$

$T_{c}$

$u$

$V V 1-V V 3$

$X$

$\mathcal{X}$

$\Delta u$

$\kappa$

$\mu$

superscript high

superscript low controller integral times

continuous optimisation parameters

simple upper bound

simple lower bound

steady state value

controller errors

product solute flow

total product flow

product water flow

controller integral actions

time derivative of $I$

controller proportional gains

scaled controller proportional gains

liquid flow valve identifiers

measured variables

effect 2 pressure

steam chest pressure

controller set points

controller parameters or effect 2 temperature

scaled controller integral action parameters

steam chest temperature

manipulated variables

vapour flow valve identifiers

product composition or integer variables

pseudo-integer variables

controller actions

scale factors

nominal valve positions

simple upper bound

simple lower bound 


\section{Chapter 1}

\section{Introduction}

... the selection of the manipulated variables, measurements and controller structure usually has a much more significant impact on the resulting closed loop performance than the design of the controller itself (Morari, 1987).

Mmmmm, fascinating. Attributed R. B. Jarvis, 1992.

Current design methods for chemical and process plants develop flowsheets based almost entirely on the steady state characteristics and economics of the processes (Douglas, 1988; Grossmann, 1990; Stephanopoulos, 1990). After generating several optimal or near optimal steady state plants, the controllability, operability, safety, health and environmental (COSHE) attributes of the flowsheets are assessed. If some plants meet the COSHE requirements laid down in the design brief, then the plant with the best economics among this subset is chosen for detailed design. If none of the plants meet the COSHE specifications, then the flowsheets are modified until they meet the COSHE requirements. The steady state economics are then reassessed and the plant with the best economics is chosen for further development.

However, current trends indicate a desire to carry out process design by considering the COSHE attributes of the plant simultaneously with the steady state plant design. The use of these factors in design can have several useful, general effects, such as reducing the lead time between plant design and plant construction and making a better cost commitment at the beginning of the design process (MacCallum, 1990).

The area of interactions between process design and process dynamics for continuous process plants is of particular interest, owing to the following factors (Lenhoff and Morari, 1982; Perkins, 1989):

1. Processes have become more tightly coupled and interactive because of (a) reduction of plant inventories to improve SHE characteristics and reduce working capital, and (b) increased process recycles to improve utilisation of energy and 
raw materials. This has led to increased importance of process dynamics in plant operation.

2. Modern process plants are operated in a dynamic environment, and are expected to handle variation in ambient conditions and management imposed demands on production.

3. A concern to avoid commissioning and operational problems that may be caused by considering only steady state modes of operation during process design.

4. Plant wide process control is of increasing interest, requiring a more general understanding of process dynamics than is required for unit operation control.

5. Process dynamics can have a significant effect on process economics. For example, improvements of $2-6 \%$ in process economics have been reported in control upgrade studies (Tyrrell, 1983; Zakrzewski, 1983; Marlin et al., 1991). If such benefits are available through retrofit studies of dynamics, this suggests that improvements of at least this magnitude should be possible by examining process dynamics during the design phase.

6. The need to spend effectively on control systems.

These factors mainly apply to continuous plants, as batch operations generally consist of either well understood, simple dynamic operations, or complex processes (e.g. batch distillation, reaction) that require a good understanding of dynamics before they can be designed. Therefore, only continuously operated plants will be considered in this thesis. The problem addressed will be further limited to considering processes that are intended to operate at steady state conditions, and only the dynamics of normal operating modes will be considered. In practice, process dynamics should be addressed for all modes of process operation, including start up, shut down, upset conditions (violating the plant operating envelope) and normal operating modes. However, this results in a problem too large to be addressed in this work.

The process disturbances may be treated as falling into the categories of regulatory and optimising disturbances, based on the frequency of the disturbances 
(Morari et al., 1980). Optimising disturbances are either steady state (e.g. change in production rate), or "slow" relative to the speed of response of the process (e.g. long term variation in feedstock quality). For these quasi-steady state disturbances it is possible to re-optimise the plant steady state operating point to take advantage of the new "steady state" operating conditions. Regulatory disturbances typically have frequencies close to the dominant process dynamics, and therefore require a regulatory mechanism to manage their effects. This thesis will focus on issues associated with regulatory control. Therefore, optimising control will not be considered. It will also be assumed that steady state is the dominant mode of operation. That is, the plant is only subject to infrequent regulatory disturbances, and so may operate at steady state for long periods of time. Given that steady state is the dominant mode of operation, then the process economics may be approximated with little error by the steady state economics. A more formal statement of this result is given in the following chapter.

Clearly the effects of process dynamics are closely related to the problem of process control system design, or control system synthesis as it is referred to by some practitioners. The control system synthesis problem may be defined as selecting the following (Nishida et al., 1981):

1. a set of control objectives

2. a set of variables to be controlled to achieve the control objectives

3. a set of variables which can be measured for monitoring the plant behaviour

4. a set of manipulated variables

5. the connections between measured and manipulated variables (control loops)

For this thesis, a control structure is defined as a set of measured variables appropriate for attaining the desired control objectives, and a set of manipulated variables appropriate for achieving the desired level of control. It does not necessarily include a control law relating the manipulated variables to the measured variables. This definition encompasses the first four aspects of control system synthesis addressed above, with control objectives included implicitly.

Most of the effort in solving process control problems has been directed at the last part of control system synthesis, the problem of designing a controller for a 
specified set of inputs and outputs, as it is most amenable to mathematical solution (Morari, 1981). However, it has been recognised that the performance of the process control system is ultimately limited by the choice of manipulated and measured variables, rather than the implemented process controller (Lee and Weekman, 1976; Morari, 1987). That is, the attainable performance of a controller is limited by the choice of control structure. This suggests that valuable contributions to the field of process control synthesis may be made by examining the problems of determination of process control objectives and selection of the corresponding control structure. This problem consists of choosing the optimal control structure (the structure that best attains the control objectives) from the set of all potential control structures for a given process (the set of all feasible combinations of measurement and manipulated variables).

Prior to discussing control objectives in detail, it should be noted that process controller robustness and stability are requirements of the controller rather than control objectives. Therefore, they will be addressed only when considering controller performance.

\subsection{Process control objectives}

An approach for determining appropriate control objectives will be developed by considering the process operating objectives and their interactions with the process dynamics. For the purposes of this discussion, operating objectives describe both process performance requirements and process objectives to be optimised. Control objectives may then be described as a subset of the operating objectives which are to be achieved using process control. Determination of the control objectives begins by considering the operating objectives for an uncontrolled plant. The operating objectives may be conveniently divided into five categories, based on those suggested by Nishida et al. (1981) for control system synthesis. These categories of operating objectives are listed below, along with typical examples:

1. Product quality specifications

- A product stream must be maintained above the minimum specified product purity 
- The quantity of a valuable reactant in purge stream must be maintained below a maximum weight percentage of the purge stream

2. Safety aspects

- The concentration of toxic components in byproduct and effluent streams must be below legal and company limits

- The inventories of hazardous chemicals on site must be maintained below legal and company limits

3. Operational requirements

- The hydrogen to aromatics ratio in the feed to a hydrogenation reactor must be kept high enough to prevent the reactor coking up

- The liquid levels in reflux drums and reboilers must be maintained between their operating limits

- Distillation column sieve trays must be operated between their flooding and weeping limits

- Minimum production rates to ensure stable plant operation must be maintained. For example, a minimum throughput may be required to operate some process units

- The rate of change of temperature in an exothermal reactor must be kept below a desired limit

- The plant must be able to switch between two operating regimes within a specified time

4. Environmental and related regulations

- The pH of effluent streams must lie between acceptable limits

- The biological oxygen demand of effluent streams must be below a specified level

- Maximum acceptable airborne pollution limits must be met

5. Economic considerations 
- Maximisation of operating profit, or minimisation of operating cost

It should be noted that there is a significant distinction between the first four categories and the last. The first four categories involve operating within some limit or constraint. That is, these operating objectives aim to maintain some quantity above or below a desired limit, implying that all four may be written in the general form:

$$
h(y) \geq 0
$$

where $y$ is a vector of process variables and $h$ is the operating objective to be maintained at a positive value. On the other hand, category 5 involves optimisation of an economic potential, i.e. the operating objectives may be written in the form:

$$
\min J\left(y_{0}\right)
$$

where $J$ is the operating objective to be minimised, and $y_{0}$ is the steady state operating point. As the economics are dominated by the steady state (see earlier discussion on regulatory/optimising disturbances), a steady state objective function is used. The steady state operating point may also be regarded as the nominal operating point, from which the process deviates during operation. The use of the above concepts results in the operating objectives being reduced into the two general categories of constraints and economics (similar to the categories of constraints and optimising objectives employed by Prett and Garcia (1988) for control system design). For the purposes of this thesis, all of the constraint operating objectives are regarded as hard constraints. That is, all of these objectives must be satisfied at all times.

The category of constraint operating objectives may be further decomposed into the subcategories of "active" and "inactive" objectives. This decomposition is demonstrated by considering the uncontrolled plant behaviour at a feasible nominal operating point $y_{0}\left(h\left(y_{0}\right) \geq 0\right)$. When the process is subject to normal process disturbances (deviates from $y_{0}$ ), a subset of the constraints, $h^{\prime}(y)$, will be violated, while the remaining constraints, $h^{\prime \prime}(y)$ are not violated. There are two possible methods of eliminating any given constraint violation in the "active" constraint set, $h^{\prime}(y)$ :

- Move the nominal operating point $y_{0}$ so that the constraint is not violated under disturbance conditions (i.e. remove the constraint from the "active" 
set)

- Add a controller to the process which reduces the variation in the constraint such that it is no longer violated under disturbance conditions

Controllers must be employed to achieve the operating objectives in cases where there is no feasible nominal operating point for which some constraint is not violated under disturbance conditions. Any objective whose constraint is controlled is self evidently a control objective. On the other hand, the "inactive" subset does not require that any action be taken, as these operating objectives are not violated during normal operation, (i.e. $h^{\prime \prime}(y) \geq 0$ remains satisfied during open loop operation). A typical example of an element of $h^{\prime}(y)$ is the objective of maintaining product purities in a high purity distillation column. The required purity can be maintained readily at steady state without control, but when the column is subject to typical disturbances, the objective will be violated if no control is applied. An example of an element of $h^{\prime \prime}(y)$ is a column whose throughputs are such that it is known that the flooding limit will never be reached. Therefore, there is no incentive to install a controller for the flooding objective as it will be satisfied with or without control.

As discussed above, the "active" constraint set will depend on the chosen nominal operating point $y_{0}$. Ideally, this point should be chosen to optimise the operating economics, leading naturally to the introduction of economic operating objectives, $\min J\left(y_{0}\right)$. Initially, assume that this objective is to be optimised ignoring process disturbances (i.e. optimising the nominal operating point), and require that the optimal nominal process vector $y_{0}^{*}$, which minimises $J\left(y_{0}\right)$, satisfies all of the constraint operating objectives. This will yield a set of "active" constraint operating objectives $h^{\prime}\left(y_{0}^{*}\right)$, that require control to maintain optimum operation. That is, the economic operating objectives can be used to choose the best "active" set of constraint control objectives. In practice, it is impossible to control the process on these constraints. Therefore, the control objective becomes minimisation of the variation in the active constraints, thereby allowing operation as close as possible to $h^{\prime}\left(y^{*}\right)=0$. The concept of controlling the process on constraints is an almost universal feature of control system synthesis methods (Morari et al., 1980; Govind and Powers, 1982; Gannavarapu, 1991; Padley, 1991), and it has been suggested that if a process is not being operated on a set of active constraints, then the plant 
contains wasteful overdesign (Lee and Weekman, 1976). It also worth noting that any implemented controller will have an associated cost. Therefore, any approach to the problem of control structure selection should address the trade off between implemented controller costs/benefits and the cost of altering the operating point to remove the constraint from the active set.

This result is similar to that obtained by considering regulatory and optimising control objectives (Morari et al., 1980; Nishida et al., 1981), where regulatory control is intended to operate the plant as close as possible to a set of constraints that are limiting process performance, and optimising control is used in a quasi-steady state fashion to alter the plant operating point with respect to "slow" or steady state disturbances, either tracking or switching the active constraints to maintain the best economics. This approach also demonstrates the strong inter-relationship between the constraint objectives and the economic objectives for well operated processes.

In summary, from consideration of the interaction between constraint operating objectives and economic objectives it is possible to determine control objectives for the control system design problem based on a subset of the active operating constraints at the optimal limits of the uncontrolled plant's operating envelope. Application of this approach to the problem of regulatory control structure selection leads to the specification of a set of constraint control objectives $h^{\prime}\left(y_{0}^{*}\right)$. Ideally, to achieve these control objectives, it would be desirable to measure $h^{\prime}(y)$ in the region of $y_{0}^{*}$. In practice these quantities are sometimes difficult to measure, and therefore practical controllers measure and control related variables to minimise the variation in the desired control objectives. The problem of which measurements and manipulated variables to employ to achieve this control is addressed by the control structure problem.

Note that this is not a comprehensive discussion of control objectives, as the above approach does not address situations where dynamic variability directly affects the process economics rather than through the active constraint set. For instance, the cases of minimising variability for quality purposes and product value varying with composition cannot be handled by the above approach as they affect the process economics directly. 


\subsection{Control structure selection and ranking}

Given that control objectives can be determined using the above reasoning, the next step in the design process is to select the "best" control structure from the space of potential structures. This is a classical synthesis-analysis design problem (Rudd et al., 1973), where a synthesis stage is required to generate a potential control structure, and an analysis technique is required to rank the structure against previously determined structures (the analysis technique supplies the measure of performance of the structure). The synthesis problem is combinatorial in nature, as process plants contain vast numbers of potential control structures. Several approaches to the synthesis stage have been advanced, ranging from engineering experience in unit operation control (Umeda et al., 1978), cause and effect analysis (Govind and Powers, 1982) and structural feasibility (Morari and Stephanopoulos, 1980a). Although all of these approaches include some analysis to limit the number of structures generated, they are largely designed to generate a set of control structures for the control engineer to rank (select the "best" structure). The issue of ranking structures is not straightforward, as there is an open ended question of what is the "best" control structure. The ranking of control structures could be achieved by designing an optimal controller for each structure and then carrying out rigorous dynamic simulation to test controller performance (Perkins, 1989). However, rigorous simulation is extremely time consuming even for small numbers of structures and so is impractical as a performance ranking method for evaluating the large number of potential control structures on a process plant. Therefore, work has concentrated on methods for the rapid ranking of control structures without designing control systems. Initial work on ranking structures concentrated on the use of heuristics (e.g. solids flows are undesirable as manipulated variables), and control theoretic arguments based on steady state gains, dead times and time constants (Umeda et al., 1978; Morari and Stephanopoulos, 1980a; Govind and Powers, 1982). More recently work on the attainability of perfect control in the forms of either dynamic resilience (Grossmann and Morari, 1984) or functional controllability (Rosenbrock, 1970; Wong, 1984; Perkins, 1989) has proved its usefulness as a ranking of control structure performance. For a specified control structure, these approaches may be used to quantify attainable control quality, sensitivity to process model errors, 
and effects of non-minimum phase behaviour and manipulated variable constraints, in the form of controllability indices. However, the controllability analyses have one significant disadvantage when used in design studies: the performance indices may not be compared directly with the process economics. This leaves the design engineer with the decision of trading off an abstract controllability index against improved steady state economics (Perkins, 1989). Both Lenhoff and Morari (1982) and Palazoglu and Arkun (1987) proposed Pareto-optimal approaches to deal with this problem, trading a dynamic performance index off against the steady state process economics. However, this approach still requires the design engineer to decide what is an acceptable loss in process economics for improved process dynamics. Clearly it would be desirable to have some indication of the effects of dynamic performance on process economics, as this would allow direct trade between the steady state and dynamic operation (Perkins, 1989).

\subsection{Thesis aims}

Based on this discussion, further study of the control structure selection problem should yield a valuable contribution to the fields of process dynamics and process design. As noted above, it would be desirable to select the economically optimal control structure of a process without designing the process controller, while maintaining good controllability characteristics. This will require examination of the effects of process dynamics on process economics, and how changes in the control structure alter these economics. This thesis then intends to address two aspects of the interactions between process dynamics and process design:

- The development of systematic methods for the selection of optimal process control structures based on process economics, where it is recognised that the process control structure provides a fundamental limit on the performance of the process control system. As the problem is combinatorial in nature, the methods address systematic generation of potential control structures and efficient search of the space of potential structures for the optimal structure. A prerequisite for this problem is the development of appropriate economic measures of control structure performance. The scope of the problem will be limited to selecting economically optimal square regulatory feedback control 
structures for processes whose operation is dominated by steady state aspects (i.e. as time, $t$, tends to infinity, the expected value of a process disturbance, $p$, tends to the steady state value of the disturbance, $p_{0}$, or $\lim _{t \rightarrow \infty} E\{p\} \rightarrow p_{0}$ ). Changes in steady state operating points and non standard modes of operation will not be considered.

- Given methods for assessing the economic effects of process dynamics, how can these methods be employed to yield improved process plant designs.

Following from these aims, the contributions to knowledge expected from this thesis are:

- An improved understanding of the effects of process dynamics on process economics. It should also demonstrate the benefits obtained by using dynamic information in process design, leading to a formal framework employing process dynamics for the design of process plants with well balanced steady state and dynamic characteristics.

- A systematic approach for selection of process control structures based on the economic effects of process dynamics, and the corresponding improvement in understanding of the effects of choice of process control structure on process economics and process control. Besides its use as a tool for evaluating dynamic behaviour for design purposes, a control structure generation method may be used as a basis for designing process control systems for plant wide control.

For the remainder of this thesis, it is assumed that the reader is familiar with systems of mixed differential and algebraic equations, and the numerical solution of such systems (Gear, 1971). A familiarity with basic optimisation theory, including linear and nonlinear programming (Gill et al., 1981; Edgar and Himmelblau, 1988), is also assumed.

\subsection{Thesis outline}

Addressing the second aim of the thesis, the next chapter outlines how existing steady state design methods may be modified to take advantage of methods for evaluation of dynamic process economics. 
Chapter 3 examines previous approaches to the problems of selecting and evaluating process control structures, and existing methods for the economic analysis of process dynamics. The benefits of nonlinear and linear dynamic analyses are discussed.

Chapter 4 is a case study demonstrating evaluation of dynamic economics based on the constraint control of a linearised process model.

Chapter 5 extends the ideas developed in the previous chapter to develop a rigorous description of the constraint control problem for a fixed control structure. This description is used to formulate a mixed integer programming (MIP) problem for the selection of the optimal control structure. Chapter 6 contains case studies on the method.

Chapter 7 considers the extension to nonlinear systems, using a mixed integer differential algebraic optimisation (MIDAOPT) formulation for the selection of optimal multiloop proportional-integral controllers. Chapter 8 considers case studies using the MIDAOPT method.

An overview of the thesis is presented in chapter 9, along with brief discussion of further areas of work. 


\section{Chapter 2}

\section{Dynamic economics in process design}

This chapter examines the requirements for designing plants with good steady state and dynamic operating characteristics (the combined design problem). This examination concludes that the combined problem is too large to address directly, and leads to a decomposition of the problem into more tractable subproblems addressing structural flowsheet optimisation and assessment of the effects of process dynamics on the process economics, hereafter referred to as dynamic economics. Finally, it is demonstrated that existing steady state process design methods may be adapted readily to employ the proposed problem decomposition.

\subsection{The process synthesis problem}

A convenient description of the combined dynamic and steady state design problem may be developed by considering the general process synthesis problem. Grossmann (1990) defines process synthesis as the generation of a process flowsheet to convert a set of specified inputs (raw materials) to a set of desired outputs (products) while meeting required processing specifications and optimising a given objective or goal function. It involves the selection of an appropriate configuration or topology of process units and their interconnections and determination of their design parameters such as process unit sizes and operating conditions. Westerberg (1980) notes that this problem may be formally represented as a nonlinear mixed integer and continuous variable optimisation problem, generally a very large one. This description leads to the superstructure view of process synthesis, where design optimisation takes place within a superstructure containing all the units capable of achieving the process goals and all the potential connections between the units. Discrete $0 / 1$ variables show the presence of units and connections in substructures generated during the optimisation, and continuous variables represent equipment sizes and process flows. The essential ingredient of this representation is that the problem consists of discrete decisions to determine the shape of the flowsheet, and 
continuous optimisation to determine the flowsheet performance.

Steady state flowsheeting methods which exploit the formalism of the process synthesis problem have been developed over the past 10 years. These design procedures aim to ensure that the space of all possible flowsheets is systematically searched for the optimum flowsheet. However, there has been little or no work on a general representation of the combined dynamic and steady state design problem. Therefore the following is an attempt to describe the problem using the structural formalism introduced previously.

\subsection{The combined design problem}

The combined design problem consists of selecting an optimal process flowsheet including unit operations and process controllers. That is, from a field of all possible processes that produce the desired material, choose the optimal process (i.e. unit operations and their connections) and the optimal controllers for the process. To develop a structural representation of the combined design problem, it is necessary to have a mathematical model of the problem. Models of chemical engineering systems are naturally represented as mixed systems of differential and algebraic equations (DAE systems) (Gritsis, 1990). Systems of partial differential equations may be reduced to DAE form by the method of lines (Carver, 1976). Along with appropriate initial conditions, the DAE system for a process operation may be written as:

$$
\begin{gathered}
f(\dot{x}, x, z, d, p)=0 \\
g^{\prime}(x, z, d, p)=0 \\
\dot{x} \in \mathcal{R}^{n_{x}}, x \in \mathcal{R}^{n_{x}}, z \in \mathcal{R}^{n_{z}}, d \in \mathcal{R}^{n_{d}}, p \in \mathcal{R}^{n_{p}} \\
f: \mathcal{R}^{n_{x}} \times \mathcal{R}^{n_{x}} \times \mathcal{R}^{n_{z}} \times \mathcal{R}^{n_{d}} \times \mathcal{R}^{n_{p}} \rightarrow \mathcal{R}^{n_{x}} \\
g^{\prime}: \mathcal{R}^{n_{x}} \times \mathcal{R}^{n_{z}} \times \mathcal{R}^{n_{d}} \times \mathcal{R}^{n_{p}} \rightarrow \mathcal{R}^{n_{z}}
\end{gathered}
$$

where $x$ is the vector of state variables (including controller states), $\dot{x}$ is the time derivative of $x, z$ is the vector of algebraic variables (including controller variables), $f$ is the vector of state equations, and $g^{\prime}$ is the vector of algebraic equations. The system parameters have been partitioned into the design vector $d$ and the disturbance vector $\boldsymbol{p}$ to simplify later problem representations. The elements of the parameter vectors must be specified to take up the degrees of freedom of the system, and may include time varying elements. The design vector $d$ contains the parameters 
that may be manipulated by the design engineer, such as equipment sizes and valve positions, while the disturbance vector contains the parameters that may not be influenced by the engineer, such as feeds from upstream plants and ambient operating conditions. The disturbances may typically be further partitioned into static and dynamic disturbances. Models of this form may be used to represent the physicochemical behaviour of process plants, such as mass and energy balances, reaction kinetics and control system behaviour. Commercial packages, such as SPEEDUP, are available to set up and solve such systems numerically (Pantelides, 1988). For design problems, the process will have an associated objective function to be optimised and a set of constraints that limit the process to safe and environmentally acceptable areas of operation, maintain the operating point within the region of model validity, and specify production requirements.

With the introduction of integer variables to describe the existence of process units and controllers, a large DAE system may be written to describe the superstructure of all possible unit operations and controllers. In the simplest case the elements of a vector of binary variables, $X$ can be set to 1 to show an item (unit operation, stream or controller) exists in a flowsheet, and 0 to show that the item does not appear in the flowsheet. The integer variables may be regarded as activating subsets of the DAE system in the optimisation, where the subsets describe the units in the current flowsheet. Assuming appropriate initial conditions at time $t=0$, this results in an mixed integer dynamic optimisation problem of the form:

$$
\left.\begin{array}{c}
\min _{d, X} E_{p \in P}\{J(\dot{x}, x, z, d, p, X)\} \\
f(\dot{x}, x, z, p, X)=0 \\
g(x, z, d, p, X)=0 \\
h(\dot{x}, x, z, d, p, X) \leq 0 \\
d^{\text {low }} \leq d \leq d^{\text {high }} \\
X \in\{0,1\}^{n_{X}}
\end{array}\right\} \forall p \in P(t), \forall t \geq 0
$$

where all of the vectors and functions have appropriate dimensions, $J$ is the process objective function whose expected value is to be minimised, $g$ is the augmented vector of algebraic relations, now including equality constraints, and $h$ is the vector of inequality constraints associated with design requirements. The augmented equality constraints typically represent controllers and relationships between the design variables, and reduce the available degrees of freedom. The vector of design vari- 
ables is subject to upper and lower bounds. The expected value of the objective function is to be minimised over all elements $p$ in the semi-infinite set of potential disturbances $P(t)$, where the elements of $P$ are bounded above and below (static disturbances have constant entries in $P$ ). Note that if the constraints describe a closed operating envelope, then the bounded input-bounded output stability of a plant satisfying the constraints is guaranteed (Stephanopoulos, 1984).

In words, the above problem aims to choose the optimal process structure (defined by the $X$ vector), and the optimal operating point of the process (specified by the design vector $d$ ). It is a highly complex optimisation problem, with a semiinfinite set of inputs (disturbances), and an infinite optimisation range (the system must satisfy the constraints from time $t=0$ to time $t=\infty$ ). However, given previous assumptions about the nature of the problem this thesis considers, the complexity of the problem may be reduced significantly. First, it has been assumed that plant operation is steady state dominated, so this mode is expected to dominate the economics. Therefore the time varying objective can be replaced with a steady state objective function with little effect on the economics. Second, it was assumed that the plant is subject to infrequent disturbances, with the expected value of a process disturbance tending to the steady state value as time tends to infinity. That is, the disturbance has an expected deviation of zero from its nominal value for long periods of time. This allows the disturbance vector to be written as the sum of a nominal (static) element and dynamic (transient) element. Based on this, we may remove the expectation from the objective function, and simply optimise the nominal steady state objective function. This gives a modified problem of the form:

$$
\left.\begin{array}{c}
\min _{d, X} J\left(x_{0}, z_{0}, d, p_{0}, X\right) \\
f(\dot{x}, x, z, d, p, X)=0 \\
g(x, z, d, p, X)=0 \\
h(\dot{x}, x, z, d, p, X) \leq 0 \\
d^{\text {low }} \leq d \leq d^{\text {high }} \\
p=p_{0}+\tilde{p}(t) \\
\dot{x}(t=0)=0 \\
X \in\{0,1\}^{n_{X}}
\end{array}\right\} \quad \forall \tilde{p}(t) \in P(t), \forall t \geq 0
$$

The initial condition is the steady state, represented by a subscript 0 . The nominal 
process $\left(p_{0}\right)$ must satisfy the constraints for all the transient dynamic disturbances, $\tilde{p}(t)$ in the set $P(t)$. This partitioning into steady state economics and dynamic operation is similar to ideas discussed in the previous chapter based on the partitioning of process disturbances into the categories of optimising (steady state economics) and regulatory (dynamic operation) disturbances (Morari et al., 1980).

Figure 2.1 is a geometric interpretation of this problem in the plane of the algebraic variables $z 1$ and $z 2$. For convenience of representation and discussion, $h 1$ and $h 2$ are shown as static constraints. The optimal solution is shown as the shaded region $A$. The central point is the optimal steady state operating point, and the shaded area is the dynamic "region" of operation around the steady state operating point. Feasible solutions to the design problem consist of the subset of integer solutions (fully specified process flowsheet and controllers) for which it is possible to generate dynamic regions which do not violate $h 1$ and $h 2$. The dynamic region is described by the responses of $f$ and $g$ to all of the possible disturbances $P(t)$. The shape, size and location of the dynamic region for any integer solution may be varied by altering the values of the continuous design parameters. The optimum is the integer solution whose optimal dynamic region, with respect to the design parameters, minimises the steady state objective function, generally corresponding to a small dynamic region as close as possible to the limiting constraints. In short, the combined design problem generates a process flowsheet whose dynamic region allows the best possible steady state operation within the process operating envelope.

The problem of control structure selection is a subset of the combined design problem proposed above, where all of the integer variables corresponding to unit operations and unit connections are fixed, and the optimisation is being carried out only with respect to integer variables describing the controller superstructure. As the control structure selection problem has already been discussed as an area still requiring significant research effort, it is argued that it is impractical to try to solve the combined design problem directly without an improved understanding of the control structure selection problem. A second, more significant argument against trying to solve the above problem directly is the sheer magnitude of the problem. In practical terms, solving a steady state problem with a superstructure as large as that expected for a typical combined design problem would prove extremely difficult using existing methods. Therefore a design decomposition is proposed to avoid the 


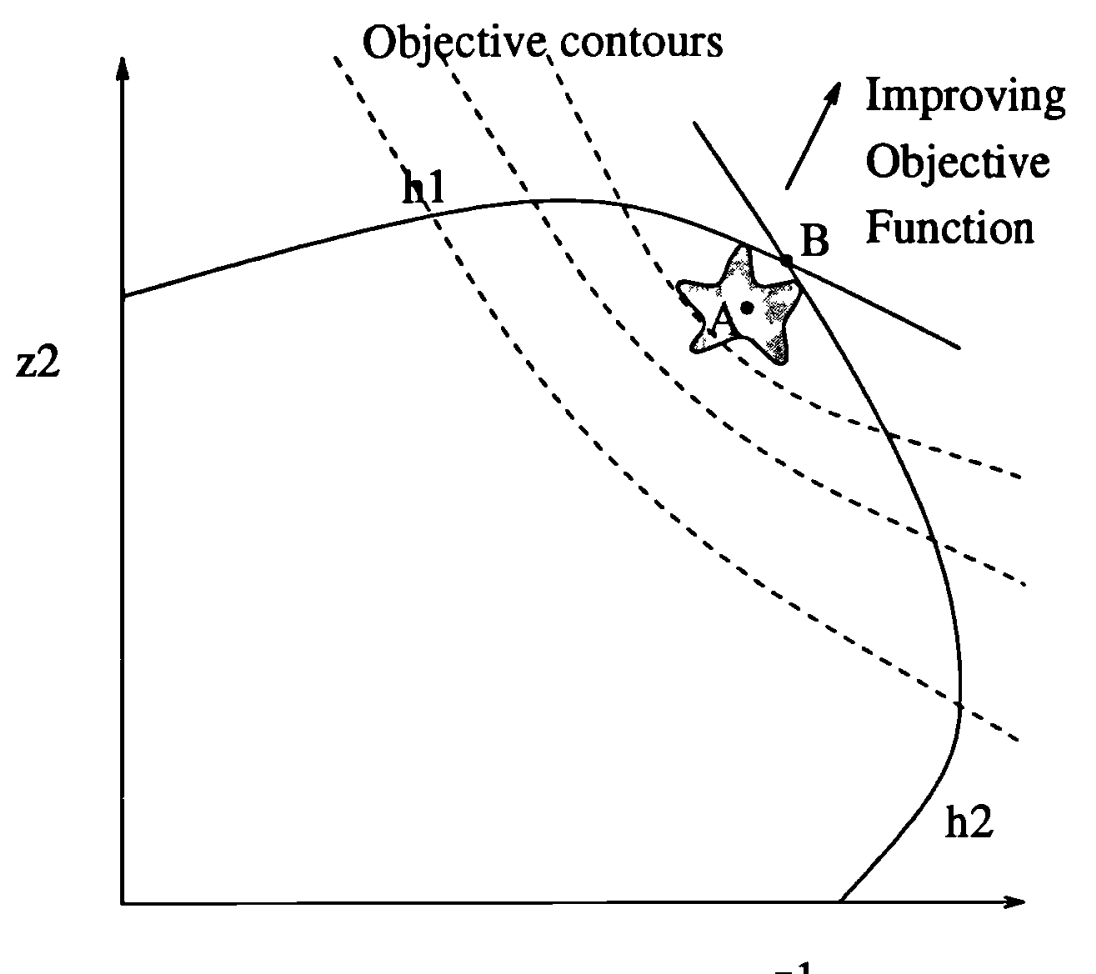

z1

Figure 2.1: Geometric interpretation of the combined design problem

difficulties associated with solution of the combined problem. The proposed decomposition allows development of methods for the assessment of effects of dynamics on process economics and provides a systematic framework for the use of these methods in process design.

\subsection{Steady state bounding of dynamic economics}

Since this thesis considers plants whose dominant mode of operation is steady state, the proposed design decomposition strategy uses steady state bounding on dynamic economics and can be incorporated readily into existing flowsheeting procedures. For a plant with fixed unit operations and connections, but unspecified controllers, the steady state bound on dynamic economics is simply stated as: The optimum nominal (steady state considerations only) objective function of the process is an optimistic bound on the attainable dynamic economics of the process, regardless of the choice of controllers. That is, the optimum dynamic economics of a process cannot be better than the optimum steady state (nominal) economics. Note that by previous assumptions, the dynamic economics implies optimising the 
steady state economics of the process subject to dynamic disturbances. It does not imply a time varying objective.

The proof of the bounding property follows from considering the design problem for a plant with a fixed process structure (flowsheet) but an unspecified control structure. Based on the material presented in the previous section, this problem may be written as:

$$
\begin{aligned}
& \min _{d, k, X} J\left(x_{0}, z_{0}, u_{0}, d, p_{0}, k, X\right) \\
& f(\dot{x}, x, z, u, d, p)=0 \\
& g(x, z, u, d, p)=0 \\
& h(\dot{x}, x, z, u, d, p) \leq 0 \\
& p=p_{0}+\tilde{p}(t) \\
& u=c(\dot{x}, x, z, u, d, p, k, X) \\
& \dot{x}(t=0)=\dot{x}_{0}=0 \\
& D\left(x_{0}, z_{0}, u_{0}, d, p_{0}\right) \leq 0 \\
& u^{\text {low }} \leq u_{0} \leq u^{\text {high }} \\
& d^{\text {low }} \leq d \leq d^{\text {high }} \\
& k^{\text {low }} \leq k \leq k^{\text {high }}
\end{aligned}
$$

where $x$ is the vector of state variables, $z$ is the vector of algebraic variables, $p$ is the deviation disturbance vector, subscript 0 refers to the nominal (steady state) values of the variables at time $t=0$, and $p_{0}$ is the nominal disturbance. The vector of integer variables, $X$, describes possible controller structures and the vector $c$ describes the behaviour of the potential controllers, where $k$ is the vector of controller parameters. The design vector has been partitioned into the vector of design variables, $d$ (constant with time), and the vector of manipulated variables, $u$ (may be time variant), both of which are subject to steady state upper and lower bounds. The controller equations use up the degrees of freedom associated with the manipulated variables $u$, but introduce further degrees of freedom in the form of controller parameters. The steady state design constraints $D$ must be satisfied at the nominal operating point, and the dynamic constraints $h$ must be satisfied at all times $t \geq 0$ for all elements $\tilde{p} \in P$. This is actually a formulation of the control structure selection problem. However, even though this problem is significantly simpler than the combined design problem, it is still impractical to solve using existing techniques, 
and is only developed for discussion of dynamic economics. Practical approaches to the control structure selection problem will require shortcut techniques for the evaluation of dynamic economics of control structures to attain acceptable solution times.

Theorem Steady state bounding of dynamic economics

$$
J_{s s}^{*} \leq J_{d y n}^{*}
$$

where $J_{s s}^{*}$ is the optimal objective function of the steady state design problem:

$$
\begin{aligned}
\min _{d, k, X} J\left(x_{0}, z_{0}, u_{0}, d, p_{0}, k, X\right) & \\
f\left(0, x_{0}, z_{0}, u_{0}, d, p_{0}\right) & =0 \\
g\left(x_{0}, z_{0}, u_{0}, d, p_{0}\right) & =0 \\
u_{0}-c\left(0, x_{0}, z_{0}, u_{0}, d, p_{0}, k, X\right) & =0 \\
h\left(0, x_{0}, z_{0}, u_{0}, d, p_{0}\right) & \leq 0 \\
D\left(x_{0}, z_{0}, u_{0}, d, p_{0}\right) & \leq 0 \\
u^{\text {low }} \leq u_{0} \leq u^{\text {high }} & \\
d^{\text {low }} \leq d \leq d^{\text {high }} & \\
k^{\text {low }} \leq k \leq k^{\text {high }} &
\end{aligned}
$$

and $J_{d y n}^{*}$ is the optimal objective function of the problem described by the system 2.2 to 2.7 .

This theorem will hold provided:

1. The controller equations do not introduce new input variables $(u)$, and may only measure and manipulate variables that already appear on the flowsheet. This condition is introduced to cover the cases such as control carried out using a trim reagent or caustic addition. In these cases the streams must be included in the process design descriptions along with appropriate bounds on the flows. The effect of this assumption is that the controller cannot change the physical structure of the process, but can only connect variables that exist in the process.

2. The simple bounds on the manipulated variables must be satisfied at all times during plant operation:

$$
u^{\text {low }} \leq u \leq u^{\text {high }}
$$


This means the controllers may not drive manipulated variables outside their normal operating region. Practically, the steady state design limits on manipulated variable action are often more generous than the dynamic limits, because of sizing limits and the desirability of avoiding large swings in control action during operation.

If these conditions are met then the steady state problem is a relaxation of the dynamic problem 2.2 to 2.7 , as the set of dynamic equations 2.3 are replaced by the steady state subset of these equations (the dynamic constraints have been relaxed to the weaker steady state constraints). Using the fact that the optimal objective function of a relaxed problem supplies an optimistic bound on the objective function of the original problem (Ahuja et al., 1989), $J_{s s}^{*}$ supplies a lower bound on $J_{d y n}^{*}$.

The bounding is demonstrated graphically in figure 2.1 , where point $B$ corresponds to $J_{s s}^{*}$ and point $A$ to $J_{d y n}^{*}$. The only effect of employing controllers on the plant is to alter the dynamic behaviour around the steady state operating point, or, the chosen controller defines the dynamic "region" of the plant. Assuming that a controller capable of perfect disturbance rejection is designed, the dynamic region of $A$ (shaded area), reduces to the steady state operating point, and $J_{d y n}^{*}$ tends to $J_{s s}^{*}$, which is a slight rewording of the bounding theorem.

\subsection{Systematic steady state design procedures}

Before developing a design procedure based on the steady state bounding property, it is worth discussing techniques for the systematic design of steady state process flowsheets. The two most notable to date are structural optimisation (Grossmann, 1990) and hierarchical flowsheeting (Douglas, 1988), both of which will be summarised here.

Structural optimisation is a literal application of the Westerberg (1980) description of the process flowsheeting problem: The design engineer proposes a superstructure containing all of the possible unit operations and connections, using continuous variables to represent the process behaviour and discrete variables to represent the structural decisions. This results in a mixed integer program, a form of optimisation problem for which standard solution techniques are available (Beale, 
1977; Nemhauser and Wolsey, 1988).

During the solution of the mixed integer program, integer solutions corresponding to full process flowsheets are generated, along with their associated economics. The process economics and the design entry cost are used to determine if the flowsheet is viable. The entry cost concept is worth brief discussion. In the course of design, whenever a new flowsheet is developed (the current flowsheet), its economics are compared to the current entry cost. If its economics improve on the entry cost, then it is the best flowsheet found to date by the design procedure. In this case, the best flowsheet is updated to become the current flowsheet, and the entry cost is updated to the economics of the current flowsheet, otherwise the current flowsheet is discarded. In either case, the design procedure then continues. In other words, the entry cost and the economics of the best flowsheet found so far are identical, and define the minimum economic performance required for any new flowsheet to become the best flowsheet found so far in the design procedure. Note that the first flowsheet developed by a design procedure is automatically the best design so far, and its economics are the initial entry cost. Integer programming techniques use the entry cost as a minimum bound on acceptable flowsheet economics to eliminate partially specified flowsheets with poor economics.

The hierarchical approach to process flowsheeting employs a hierarchy of increasing process complexity to generate a preliminary flowsheet. Alternative flowsheets are then developed in an evolutionary fashion by systematically modifying the preliminary flowsheet to examine alternative process routes at all levels of the hierarchy. This approach also uses the idea of an entry cost. The process economics of the preliminary flowsheet determine the initial entry cost. The procedure then uses this entry cost to eliminate partially specified alternatives (corresponding to the lower levels of the hierarchy) with poor economics, and updates the entry cost as flowsheets with better economic performance are generated.

For the purposes of this thesis, the most important aspect of these design techniques is that they generate several alternative process flowsheets and entry costs during the design process (either integer solutions or alternative designs). This may be exploited in conjunction with steady state bounding of dynamic economics to decompose the combined design problem into the more tractable subproblems of steady state process design and examination of dynamic economics. The decomposi- 
tion consists of using a steady state method to generate alternative flowsheets, which are then analysed for their dynamic economics. The decomposition requires tools for the calculation of the dynamic economics of a specified flowsheet (i.e. $J_{d y^{n}}^{*}$ ). It will simply be assumed the tools are available, as the practical calculation of dynamic economics will be addressed along with the control structure selection problem in the remainder of the thesis. The rest of this chapter discusses a formal basis for this decomposition.

\subsection{Decomposition based combined design procedures}

Given that tools for the evaluation of dynamic economics exist, a combined design procedure based on the steady state bounding property is as follows:

1. Using a conventional steady state design procedure, generate an initial flowsheet.

2. Assess the dynamic economics of the initial flowsheet. These dynamic economics become the entry cost for further steady state designs. For any further designs to be considered viable, they must have steady state economics at least as good as the current entry cost. This is because the steady state economics are an optimistic bound on the dynamic economics by the above theorem. Make the initial design the best design.

3. Continue with the steady state design procedure until a new design is generated. If its optimum steady state economics are worse than the entry cost, then reject the design. Otherwise evaluate the dynamic economics of the new design and compare them with the current entry cost. If they are better than the entry cost, then replace the entry cost with the dynamic economics of the new design, and replace the current best design with the new design.

4. Return to step 3 if all designs have not been considered.

The steady state design method can be any systematic method for generating flowsheets such as MINLP (Grossmann, 1990) or hierarchical design (Douglas, 1988). Once the steady state design method generates a candidate flowsheet with economics better than the entry cost, the flowsheet can be passed to a dynamic 
design package that generates the optimum economics and plant parameters under disturbance conditions. As a result of this decomposition, the dynamics of each flowsheet are considered in isolation, removing the requirement to optimise the steady state and controller superstructures simultaneously. This results in more manageable subproblems in the design procedure. While this approach is not the most efficient, it does guarantee the optimal solution to the problem (subject to the effectiveness of the steady state and dynamic methods employed), and it is convenient for the purposes of this thesis.

The above algorithm will now be formalised, along with a design filtering algorithm for generating a small number of plants with good potential economics using less effort than is required for a full combined design.

\subsubsection{Algorithm 1: Optimal steady state/dynamic flowsheet}

This is simply a formalisation of the decomposition proposed above. It is intended to generate the process design with optimal steady state and dynamic characteristics from the field of potential processes. This approach requires that the dynamic economics must be determined for each steady state flowsheet that attains the entry cost.

Assuming cost minimisation, define:

$I$ as the index of the most recent flowsheet generated by the design procedure

$J_{s s}^{I}$ as the optimum steady state objective function of $I$

$J_{d y n}^{I}$ as the dynamic optimum objective function of $I$

$\Theta$ as the index of the best flowsheet located so far in the design procedure

$J_{d y n}^{\Theta}$ as the dynamic optimum objective function of $\Theta$

i) Generate an initial flowsheet $I$, Set $J_{d y n}^{\Theta}=J_{d y n}^{I}$ and $\Theta=I$

ii) Do steady state design procedure

Until either a new flowsheet $I$ is found with $J_{s s}^{I}<J_{d y n}^{\Theta}$

(i.e. use $J_{d y n}^{\ominus}$ as an entry cost for designs)

Or the design procedure terminates (when no further flowsheets with economics better than the entry cost can be generated) 
iii) Calculate $J_{d y n}^{I}$ for the new flowsheet

If $J_{d y n}^{I}<J_{d y n}^{\Theta}$ then

Set $J_{d y n}^{\Theta}=J_{d y n}^{I}$ and $\Theta=I$

That is, replace current best flowsheet with $I$.

\section{End if}

iv Return to step ii)

\subsubsection{Algorithm 2: Optimal open loop design set}

The ideas developed in this chapter have included the implicit assumption that evaluation of the dynamic economics includes determination of the optimal control structure. Now, it should require significantly less effort to generate the dynamic economics for an open loop plant, as this will not require any structural decisions to be made for the control system. This may be used as the basis of a screening algorithm to eliminate processes with poor economics without carrying out a full dynamic economic analysis of each process which achieves the entry cost. The aim of the algorithm is to generate the process flowsheet with the best open loop economics, and a design set of processes whose steady state economics are better than the best open loop economics. As the open loop economics supply a pessimistic bound on the dynamic economics, the design set consists of all flowsheets whose optimistic economic bounds (steady state) are greater than the best pessimistic bound (best open loop economics). This requires a slight modification to the previous algorithm: The dynamic economic analysis to determine the entry cost is replaced by the simpler open loop economic analysis, and instead of simply generating a single optimal design, all designs with steady state economics better than the entry cost are retained for further examination, as they all have the potential to be the optimal flowsheet. This approach should result in the generation of a small number of flowsheets for further dynamic analysis with less effort than is required for a full application of algorithm 1. Ranking the design set in order of best open loop economics supplies a good starting point and a reduced search space for algorithm 1.

Retaining the previous definitions, define: 
$J_{o l}^{I}$ as the optimum objective function of $I$ taking open loop dynamics into consideration

$J_{o l}^{\Theta}$ as the optimum objective function of the best flowsheet found to date taking open loop dynamics into consideration

$\Lambda$ as the open loop design set (flowsheets which have steady state optimum objective functions better than $J_{o l}^{\Theta}$ )

i) Generate a candidate flowsheet $I$, Set $J_{o l}^{\ominus}=J_{o l}^{I}$ and $\Lambda=\{I\}$

ii) Do steady state design procedure

Until either a new flowsheet $I$ is found with $J_{s s}^{I}<J_{o l}^{\Theta}$

(i.e. use $J_{o l}^{\ominus}$ as an entry cost for designs)

Or the design procedure terminates (when no further flowsheets with economics better than the entry cost can be generated)

iii) Calculate $J_{o l}^{I}$ for the new flowsheet

If $J_{o l}^{I}<J_{o l}^{\Theta}$ then

Set $J_{o l}^{\Theta}=J_{o l}^{I}$ and $\Lambda=\left\{i \in \Lambda \mid J_{s s}^{i}<J_{o l}^{\Theta}\right\} \cup\{I\}$

That is, remove all flowsheets from $\Lambda$ with $J_{s s}^{I}$ worse than $J_{o l}^{\Theta}$

Else

Set $\Lambda=\Lambda \cup\{I\}$. That is, add flowsheet $I$ to $\Lambda\left(J_{s s}^{I}\right.$ is automatically less than $J_{o l}^{\Theta}$ for $I$ to be considered for dynamic analysis)

\section{End if}

iv) Return to step ii)

\subsubsection{Example}

A simple, artificial example is discussed here to demonstrate the proposed design algorithms. The algorithms will be applied to the eight plants summarised in table 2.1. The plants are generated in this order by a steady state design procedure (considering cost minimisation).

Examining the results, flowsheet 4 is the steady state optimal flowsheet, and flowsheet 3 is the dynamic optimal flowsheet. Although this is an artificial example, this simply serves to remind that the steady state optimal flowsheet may not be the dynamic optimal flowsheet. 


\begin{tabular}{|c|c|c|c|}
\hline Plant & $\begin{array}{c}\text { Steady state } \\
\text { optimum }(£)\end{array}$ & $\begin{array}{c}\text { Open loop } \\
\text { optimum }(£)\end{array}$ & $\begin{array}{c}\text { Dynamic } \\
\text { optimum }(£)\end{array}$ \\
\hline 1 & 5,800 & 6,800 & 5,900 \\
2 & 2,200 & 4,000 & 2,400 \\
3 & 1,900 & 3,600 & 2,100 \\
4 & 1,600 & 3,000 & 2,400 \\
5 & 5,600 & 5,900 & 5,700 \\
6 & 5,500 & 6,000 & 5,700 \\
7 & 1,800 & 3,300 & 2,800 \\
8 & 6,300 & 7,500 & 6,500 \\
\hline
\end{tabular}

Table 2.1: Design decomposition example

Considering the application of algorithm 2 first, the initial flowsheet 1 and its open loop economics are generated. The entry cost is set to the open loop economics of flowsheet 1. The next flowsheet generated (2) has better open loop economics than the entry cost, so the entry cost is updated to this value. Flowsheet 1 is removed from the design set as its steady state economics are worse than the new entry cost. The next two designs are added to the design set, and the entry cost is updated each time. Flowsheets 5,6 and 8 are eliminated as their steady state economics are worse than the entry cost $(£ 3,000)$. Flowsheet 7 is added to the design set, but the entry cost is not updated as its open loop economics are worse than the entry cost. The resulting design set consists of flowsheets $2,3,4$ and 7 .

Algorithm 1 operates more directly, with flowsheet 2 eliminating flowsheet 1 after examining the dynamic economics, and flowsheet 3 eliminating flowsheet 2 in a like manner. As flowsheet 3 is the optimum flowsheet, the remaining flowsheets are eliminated either by steady state bounding $(5,6$ and 8$)$ or by dynamic economic analysis (4 and 7). Here, the optimal flowsheet is generated by designing eight steady state flowsheets, and carrying out dynamic analysis on five of the flowsheets.

\subsection{Summary}

This chapter started by considering the problem of designing processes with good dynamic and steady state operating characteristics. It was concluded that this problem is too large to solve directly, requiring decomposition into the two smaller problems of process structure selection using steady state methods and analysis of 
dynamic economics for a fixed process structure. The two problems are related by using the dynamic economics to generate entry costs for the structural flowsheet analysis. The advantages of the decomposition approach are:

1. The dynamic economics need not be calculated for all of the flowsheet structures generated.

2. The subproblems are far more tractable than a complete combined design problem solution.

One of the aims of this thesis was to develop a suitable systematic framework for the employment of process dynamics to improve process designs. This framework is supplied by the two design algorithms developed in this chapter. These algorithms have the added advantage that existing steady state design methods may be readily modified to employ the algorithms, provided tools are available to assess the dynamic economics. Assessment of the dynamic economics will be the subject of the remainder of the thesis. 


\section{Chapter 3}

\section{Historical review}

This chapter reviews relevant topics for the control structure selection problem, covering historical approaches to the problems of control structure generation and analysis, and approaches for the evaluation of dynamic effects on process economics. The relative advantages and applicability of the methods are discussed, and any techniques with direct application to this thesis are highlighted. The chapter concludes with a discussion of the relative benefits of linear and nonlinear dynamic analysis. This discussion leads to preliminary approaches for the solution of the control structure selection problem.

\subsection{Control system synthesis}

Control system synthesis is concerned with the systematic design (selection and parameterisation) of process control systems, as opposed to ad hoc methods for process controller design. The need for systematic control system design was recognised as early as 1964 by Buckley (1964), who decomposed the control objectives into two categories: material balance control; and quality control, and then showed that the two types of control are concerned with low frequency and high frequency dynamics respectively. It was demonstrated that this decomposition can be imposed on process plant by the installation of intermediate storage capacity. More recent approaches have taken more consideration of the actual control objectives (e.g. process optimisation, quality control, constraint control), generally at a plant wide level. The control system synthesis problem has recently been by reviewed by Nishida et al. (1981), Stephanopoulos (1983) and Russell (1987). It was noted in Chapter 1 that control system synthesis encompasses the process control structure selection problem. Therefore, this review discusses the merits of the various control system synthesis methods with respect to the control structure selection problem. The review specifically addresses each of the methods for the following desirable attributes in control structure selection (summarised from chapter 1): 
- The selection of control objectives

- Ranking of control structures based on operating economics, preferably without designing controllers

- Efficient searching of the space of potential structures

The problem of synthesis of multiloop single input single output pairings

will not be reviewed here, as it is concerned with the interconnections between a specified set of manipulated and measured variables, rather than the selection of the measured and manipulated variables from the space of possible measurements and manipulations. The interested reader is referred to the recent review by Lie and Balchen (1991).

\subsubsection{Steady state control structure selection}

Fisher, Doherty and Douglas (Fisher et al., 1988a; Fisher et al., 1988b; Fisher et al., 1988c) developed a procedure for generating steady state control structures for preliminary flowsheets generated by Douglas' hierarchical design procedure (Douglas, 1988). These control structures are generated by considering the limiting operating constraints and the economically significant optimising variables (e.g. reactor conversion, flash temperature) of each preliminary flowsheet, referred to collectively as the operating variables of the flowsheet. For on-line steady state optimisation, it is desirable both to optimise the significant optimising variables and to operate on the limiting constraints. To satisfy the steady state degrees of freedom for this goal requires at least one manipulated variable for each operating variable (i.e. at least one manipulated variable for each variable optimised).

The first article outlines a procedure for ensuring that the steady state degrees of freedom are satisfied. At each level of the design hierarchy, if the number of manipulated variables is greater than or equal to the number operating variables, then the procedure continues to the next level of the hierarchy. Otherwise, either the cheapest combination of the following actions is taken:

1. Modify the flowsheet to introduce new manipulated variables (e.g. heat exchanger bypass streams)

2. Overdesign some process items to reduce the number of limiting constraints 
3. Do not control the economically least significant optimising variable

or, the analysis proceeds to the next stage of the hierarchy, and the procedure is repeated. Application of this method ensures sufficient degrees of freedom are available for steady state operating optimisation.

The second article considers systematic overdesign of the flowsheet to ensure that it is steady state operable with respect to the expected range of process disturbances. The steady state effect of the disturbances on process economics is assessed. Disturbances that alter the process economics by less than $2 \%$ are treated as insignificant, and are not considered further. Consideration of the economic and constraint behaviour with respect to each significant disturbance leads to an approximately optimal level of overdesign for the limiting process units. The level of overdesign is approximately optimal, as the procedure considers overdesigning nominal plant, rather than re-optimising the plant for the disturbance conditions. The optimal overdesign policy corresponds to one of the following: the base case design, the "worst" case design, or a design between these cases. Heuristics and shortcut calculations are used to determine the appropriate level of overdesign, with preference given to choosing either the base case or worst case designs, rather than an intermediate design (i.e. intermediate designs are only developed if both the base case and the worst case designs have poor economic performance). This analysis is strictly a flexibility analysis (Grossmann et al., 1983) to ensure the steady state operability of the process. It is simpler and faster than the approach proposed by Grossmann et al. (1983), with a corresponding loss of rigour. However, it is posed as a shortcut method to identify where overdesign is required, and if a full flexibility analysis is required.

The overdesign built into the plant in the previous stage results in excess operating capacity for the plant. The final article considers the selection of a set of steady state controlled variables to take advantage of this excess capacity. The stated aim of the steady state control system is to give near to optimal steady state performance after dynamic transients have decayed. This is achieved by carrying out a steady state operating optimisation, where the capital costs from the previous design calculations are neglected. This alters the optimal operating point for the plant to take advantage of the excess capacity, and typically results in constrained operation. Heuristics to help identify the active constraints are developed. To main- 
tain operation at this optimal level, the active constraints are selected as controlled variables in a regulatory control system. The use of steady state techniques to eliminate poor controllers (both multiloop and MIMO) are discussed briefly. Finally, the article notes the need to evaluate dynamic performance and stability of the proposed control structures.

Although this procedure primarily addresses steady state operability and control, it emphasises the requirements of appropriate overdesign for operability and the need for sufficient degrees of freedom to achieve the desired level of control. It also re-emphasises the desirability of operating on active constraints, and the trade off between controlling on the constraint or backing away from the constraint (features identified in Chapter 1 when considering the control objective problem). The method suffers from several disadvantages for the control structure selection problem. The most significant of these is the fact that it does not consider process dynamics, which are of prime importance in the development of regulatory control structures. The method also considers modification of the flowsheet to ensure sufficient degrees of freedom for operation, whereas most complete flowsheets will contain more potential degrees of freedom than desired control variables. That is, a typical plant will have more locations for valves/control loops than the number of variables in the final control system. Using this approach, it is quite possible that better choices of manipulated variables may be missed in the final control structure. That is specifying the manipulated variables incrementally may result in a poor choice compared to choosing the manipulated variables from the completed flowsheet. The prediction of the control objectives is based on heuristics, and are therefore likely to be suboptimal, and the procedure does not recognise that it will not always be possible to measure the active constraints.

\subsubsection{Multilevel synthesis}

Umeda, Kuriyama and Ichikawa (Umeda et al., 1978) propose a control system synthesis technique based on a hierarchical decomposition. The method is developed by noting that the problem of synthesising a plant wide control system is an extremely large and poorly understood combinatorial problem, whereas the problem of control system synthesis for a single unit operation is a better understood problem with low combinatorial complexity. Therefore the plant wide control system 
synthesis problem is decomposed into the subproblems of synthesising unit operation control systems and the co-ordination of the individual control systems (shown in figure 3.1). The procedure assumes that the control objectives are known, and have been converted into objectives for the process units.

At the first level of the decomposition, "optimal" controllers $C_{n j}$ are designed for each unit operation $n=1,2, \ldots, N . C_{n j}$ is the $j$ th alternative control system generated for unit $\mathrm{n}$, and is chosen to optimise the $J_{n}$, the objective function describing the controller performance with respect to the desired control objectives. Umeda et al. (1978) discuss $C_{n j}$ made up from the set of possible single loop controllers $c_{n 1}, c_{n 2}, \ldots, c_{n L}$, but this could be extended to consider all possible MIMO controllers. The makeup of the "optimal" control system $C_{n j}$ is guided by: heuristics based on the process economics and characteristics (time lags, gains, sensitivities, rangeabilities) and steady state and dynamic simulation.

Once control systems have been designed for each unit operation, the procedure transfers to the second level. At this point, the procedure identifies all streams that are being used as manipulated variables by more than one unit (i.e. control conflicts). The conflicts are resolved by modifying both control objectives and controllers to optimise an overall objective function, $J_{T}$ (the co-ordinating function). This results in revised control systems $C_{r, n j}$. The procedure terminates if there are no conflicts to resolve, or if the revised control system is acceptable. Otherwise the iteration counter $j$ is increased and the procedure returns to the first level to generate a new set of alternative controllers.

This procedure recognises the combinatorial problem of control system synthesis, and proposes a far smaller problem by considering the design and coordination of single unit operation control. However, this decomposition automatically excludes a large number of potential control structures as it is purely based on convenience of calculation, and therefore it is not guaranteed to generate the optimal control system. As it also relies heavily on heuristics at both levels of the hierarchy, it is not guaranteed even to locate the optimal co-ordinated control system. Finally, the co-ordination task is poorly defined (Stephanopoulos, 1983). 


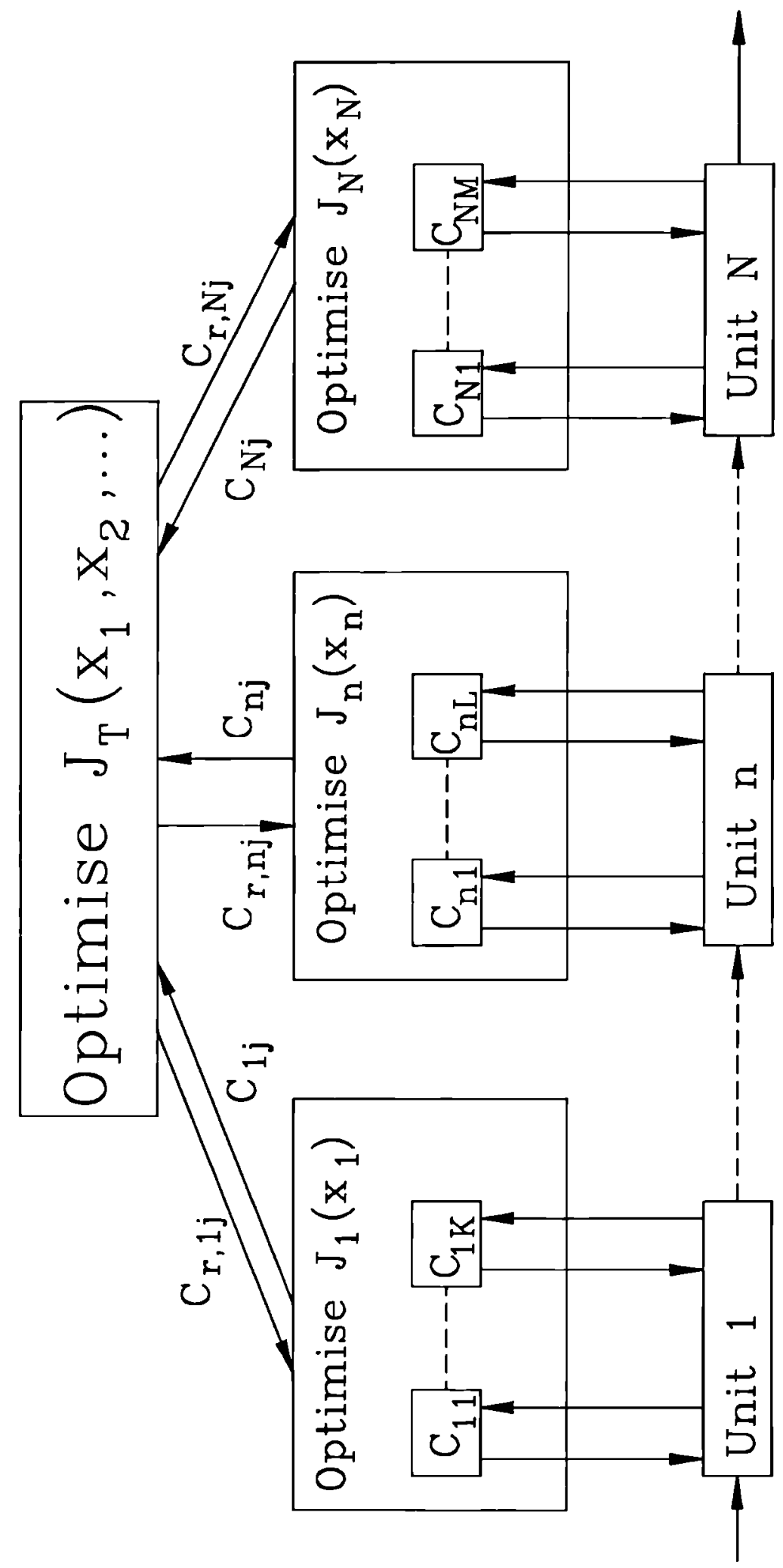

Figure 3.1: Hierarchical control system synthesis structure 


\subsubsection{Cause and effect digraphs}

The control system synthesis approach proposed by Govind and Powers (1982) uses cause and effect analysis of the process flowsheet to generate process control structures. The procedure uses similar arguments to those developed in chapter 1 to describe process control objectives as constraint objectives. These constraints are defined as constraint variables, that are to be maintained within given bounds of the steady state value for optimum economic operation. After identification of the constraint variables, the procedure consists of three stages:

1. Generation and selection of possible ways of measuring the constraint variables, both by primary and secondary (inferential) measurements

2. Generation and selection of possible ways of manipulating the constraint variables

3. Combination of the first two stages to generate feedback/feedforward process control structures

The procedure uses two structural representations of the process in the forms of structural arrays and digraphs. A structural array consists of an array of zero and nonzero elements describing the functional relationships of the process variables based on the linear transfer function between the process inputs and outputs. A nonzero element (" $X$ ") corresponding to a process variable (column) is placed in each equation (row) that the variable occurs. Zero elements are typically not represented. As the array is based on the process transfer function, each row contains one output element and elements for all of the inputs that affect the output. The structural array is also referred to as the occurrence matrix (Russell, 1987). To demonstrate the concept, the structural array for the liquid-liquid heat exchanger in figure 3.2 is shown in table 3.1 (taken from Govind and Powers (1982)), where M and $\mathrm{T}$ are mass flows and temperatures with numbers referring to streams.

Cause and effect digraphs are generated to show causal relationships between process variables. Digraphs consist of a set of nodes describing each of the process variables, with directed edges showing that altering the variable will cause changes in the variables at the end of the edges out of the altered variable node. For example, returning to the liquid-liquid heat exchanger (figure 3.2), modifying 

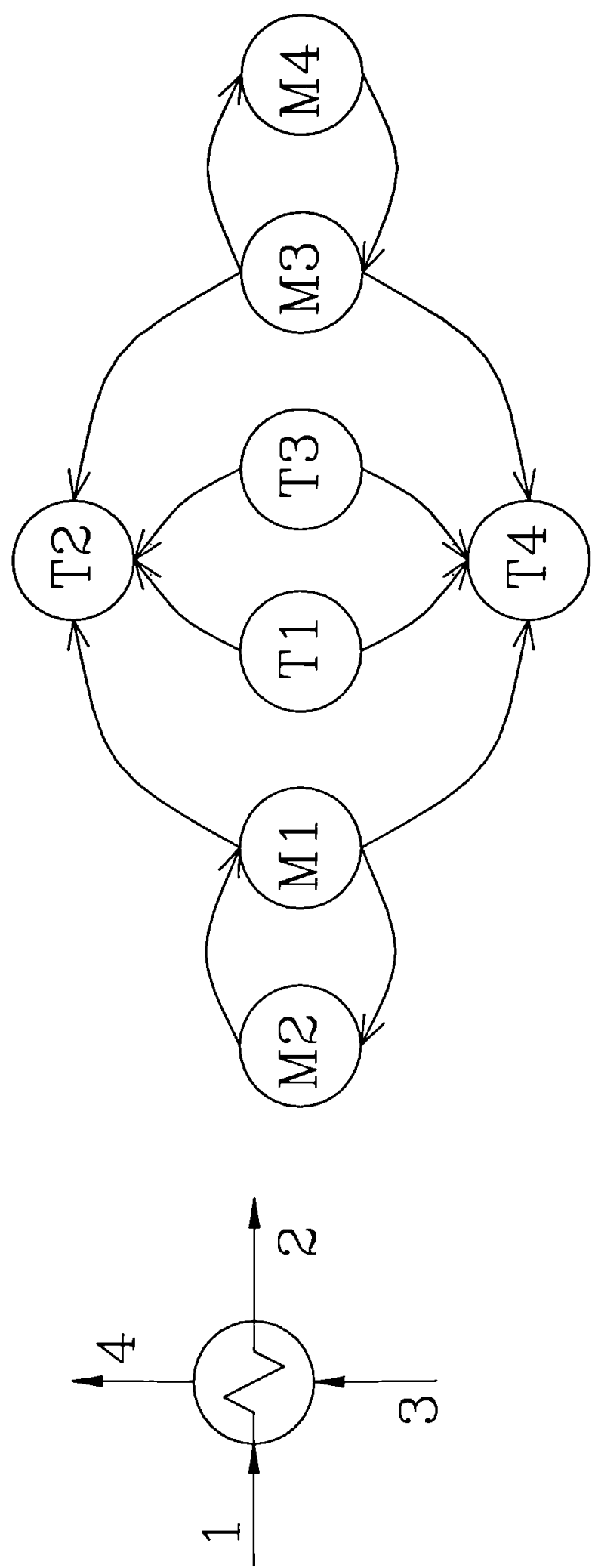

Figure 3.2: Liquid-liquid heat exchanger and associated digraph 


\begin{tabular}{|c|cccc|cccc|}
\hline & \multicolumn{5}{|c|}{ Inputs } & \multicolumn{4}{c|}{ Outputs } \\
& $\mathrm{M} 1$ & $\mathrm{M} 3$ & $\mathrm{~T} 1$ & $\mathrm{~T} 3$ & $\mathrm{M} 2$ & $\mathrm{M} 4$ & $\mathrm{~T} 2$ & $\mathrm{~T} 4$ \\
\hline 1 & $\mathrm{X}$ & & & & $\mathrm{X}$ & & & \\
2 & & $\mathrm{X}$ & & & & $\mathrm{X}$ & & \\
3 & $\mathrm{X}$ & $\mathrm{X}$ & $\mathrm{X}$ & $\mathrm{X}$ & & & $\mathrm{X}$ & \\
4 & $\mathrm{X}$ & $\mathrm{X}$ & $\mathrm{X}$ & $\mathrm{X}$ & & & & $\mathrm{X}$ \\
\hline
\end{tabular}

Table 3.1: Heat exchanger structural array

mass flow M1 alters mass flow M2 and temperatures T2 and T4, and M1 is altered by modifications in mass flow M2. Feed temperatures T1 and T3 are set by the environment, and thus can only cause changes in the process, but are not subject to changes caused by the process (no directed edges enter nodes T1 and T3). Each directed edge is associated with the steady state gain, time delays and time constants of the transfer function between the variables connected by the edge.

A library of these structural models can be developed for various process units, and flowsheets descriptions built up by connecting unit operation elements.

\section{Sensed variable selection}

Given the structural representation, alternative sets of measurement variables may be generated from a "sensed" variable tree. The tree begins with a constraint variable, and alternative constraint variable sets are generated by logical AND and OR operations based on the process structure. In the heat exchanger example, if M4 is a constraint variable, and if it can be measured directly, then M4 is the first alternative measurement. The digraph shows M3 and M4 have a functional relationship, therefore M3 may be chosen as an alternative measurement (OR). Using equation 3, an alternative measurement is to infer M3 using the set $\{\mathrm{M} 1, \mathrm{~T} 1, \mathrm{~T} 2, \mathrm{~T} 3\}$ (OR with respect to $\mathrm{M} 3, \mathrm{AND}$ to generate the inferred measurement). This procedure leads to the sensed variable tree shown in figure 3.3, that may be extended to generate all possible sets of measured variables for the process flowsheet. The set of potential measured variables is limited by heuristics based on the sensor costs, accuracy and sensitivity to noise, along with the computational difficulty of calculating the inferred measurements, leading to a small number $(5-10)$ of measured variable sets for further consideration. 
The final candidate measured variables are classified as upstream measurements if they have a causal effect on the constraint variable, and as downstream measurements if they are causally affected by the constraint variable. Upstream measurements are employed in feedforward structures, and downstream measurements are employed in feedback structures.

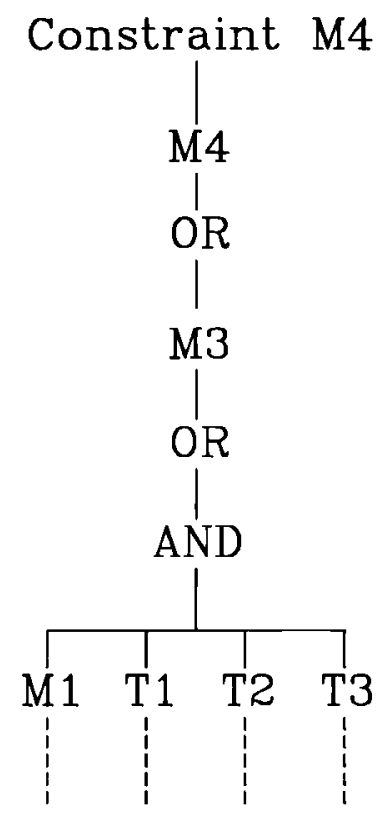

ETC.

Figure 3.3: Sensed variable tree

\section{Manipulated variable selection}

Feedback manipulated variables are generated from downstream measurement sets in a similar fashion to the sensed variables, using a "subgoal" tree to generate alternative manipulated variables that will be able to control the measured variables. The subgoal tree starts from the measurement set and generates all possible sets of manipulated variables that can control the measurement set, using AND and $\mathrm{OR}$ operations as above. The selection of the final manipulated variable sets is based on heuristics and user defined limits on acceptable steady state gains, time constants and time delays between the manipulated and the measured variables. The values of the limits are based on experience and trial and error. 
Feedforward control structures are generated in similar fashion, using the feedforward measurements as the starting points for subgoal trees.

The final stage of the procedure consists of cascading feedforward and feedback structures to improve the overall control by replacing manipulated variables from one control scheme with the set point from another.

This procedure represents a systematic approach to the generation of control structures by employing structural descriptions of the process. In particular the concept of generating measured and manipulated variable sets by inferencing from the constraint variables is worth further consideration. However, the approach suffers from a number of disadvantages for the control structure selection problem under consideration in this thesis:

- It is only suitable for the generation of multiple single loop control structures, rather than general MIMO control structures

- It does not consider economics

- It relies heavily on heuristics, and therefore cannot guarantee location of an "optimal" control structure

- It cannot identify structurally uncontrollable structures (see next section)

- It is not easily automatable

In spite of these drawbacks, the approach represents a significant advance in approaches to control system synthesis, and any further work should include structural descriptions and consider the use of limiting constraints to generate measured and manipulated variable sets.

\subsubsection{A unified, systematic approach}

Stephanopoulos and colleagues propose a comprehensive, systematic approach to the problem of control structure selection in a series of five papers entitled "Studies in the synthesis of control structures for chemical processes", referred to as Parts I to V hereafter (Morari et al., 1980; Morari and Stephanopoulos, 1980a; Morari and Stephanopoulos, 1980b; Arkun and Stephanopoulos, 1980; Arkun and Stephanopoulos, 1981). The procedure aims to generate control structures consisting of: 
1. A set of variables which are to be controlled to achieve specified objectives

2. A set of variables which can be measured for control purposes

\section{A set of manipulated variables}

4. A structure interconnecting the manipulated and measured variables

The procedure considers the synthesis of both regulatory and economic optimising control structures that are feasible from a mathematical and engineering point of view.

Part I discusses the formulation of the problem, and its decomposition into decentralised optimising and regulatory control tasks by a multilayer-multiechelon approach. Two categories of control objectives are recognised: operational feasibility and economic. Operational objectives correspond to variables that must be maintained within specified bounds to maintain feasible operation of the process. Regulatory control is used to treat these objectives (discussed in Part II). If any manipulated variables remain after development of the regulatory structure, they may be used to control the economic objectives using an optimising control strategy (i.e. feasible operation must be ensured before optimisation can be considered).

If sufficient degrees of freedom are available for optimising control, the next stage decomposes the process disturbances into slow and fast categories, where slow disturbances have periods significantly longer than the process time constant. As the process responds faster than the slow disturbances affect the plant, the slow disturbances may be treated as "steady-state" disturbances. This implies that the plant operating point can be altered to take advantage of the quasi-steady state changes in operating conditions by optimising the steady state economics. That is, slow disturbances lead to steady state optimising control structures. However, not all slow disturbances have a significant effect on process economics. A Lagrangian analysis is developed to further decompose the slow disturbances into economically important and unimportant categories. The latter disturbances and the fast disturbances are managed by the regulatory control system, while the economically significant slow disturbances are used as measured variables in steady state feedforward (optimising) structures. This decomposition of the disturbances generates multilayer control systems in the multilayer-multiechelon procedure, where the op- 
timising control structures pass set points to the regulatory control system as the operating point changes.

The remainder of Part I considers the decomposition of the steady state optimising control structure into decentralised functional units (the multiechelon stage). The decentralisation aims to develop groups of process units (e.g. a distillation train with a specific functional goal) that can be optimised independently in response to the process disturbances, in such a way that the interaction and impact of the disturbances on other groups is minimised. Initially, decentralised feedforward control of the significant disturbances is considered. Two criteria are used to determine if the decomposition is acceptable: the degradation in economic performance compared to perfect, centralised optimising control, and whether the performance is better than no control at all. The procedure aims to generate the configuration that minimises the degradation with respect to perfect centralised feedforward control. At this stage, the control objectives are re-assessed to determine if some of the feedforward objectives can be translated into regulatory feedback objectives. This may be done if some constraints are active regardless of disturbance conditions. The steady state economics of the feedback/feedforward optimising structures are also assessed against ideal centralised control and no control, and again the best configuration is accepted if the degradation in the objective function is acceptable (i.e. the structure is only accepted if the degradation is less than that required to implement either centralised control, or the best decentralised pure feedforward control structure). This is similar to the idea of constraint objectives discussed in chapter 1.

Parts IV and V discuss the design of steady state optimising control systems for functional units, and the co-ordination of the decentralised controllers for plant wide optimising control. The procedure includes consideration of smooth and efficient transfer between steady state operating conditions. These will not be discussed in any more detail, as we are considering regulatory process operation at a single operating point.

Part II is concerned with the generation of feasible regulatory control structures for the operational feasibility and feedback optimising objectives developed in Part I. The procedure assumes that the control objectives developed in Part I may be measured directly for the purposes of generating control structures. If this is not 
the case, Part III outlines a procedure for generating secondary measurements to estimate the primary measurements (control objectives). The approach consists of generating a set of measurements that will minimise the effects of estimation error and unmeasured disturbances on the inferred measurement. As this thesis is concerned with the generation of square feedback control structures, rather than the selection and design of state estimators, this approach will not be reviewed further.

For the manipulated variable selection problem, Part II notes that it would be undesirable to generate all possible sets of manipulated variables due to the large size of the resulting screening problem (q.v. Govind and Powers (1982)). To avoid this problem, the following decomposition of the regulatory control structure selection problem is proposed:

1. Group the process units into irreducible subsystems (generally units connected by recycles), and sequentially order these subsystems

2. "Tear" the subsystems into functional units, where functional units comprise a set of process units with a specific functional goal, e.g. a distillation train carrying out a specified set of separations, or a reactor with associated feed effluent heat exchanger

3. Considering each functional unit in sequence, generate all structurally feasible control structures that can meet the functional unit's control objectives, and select the best structure. The procedure includes modification of the control objectives to ensure sufficient manipulated variables are available for control

4. If control conflicts remain after generating control structures for all of the functional units, an iterative modification is carried out

This procedure is similar to the hierarchical decomposition proposed by Umeda et al., however this decomposition is based on the regulatory control objectives (leading to functional units), rather than on simplicity of calculation. The iterative procedure also allows more flexibility in the functional unit control objectives than the Umeda et al. procedure.

Generation and selection of the best control structure for each functional unit (step 3) is carried out in two stages: screening of all possible structures to eliminate infeasible structures using structural controllability criteria, and selection 
of the best structure from the feasible structures using heuristics based on the process characteristics (q.v. Govind and Powers (1982)), followed by dynamic simulation when the set of alternative structures is sufficiently small.

Selection based on heuristics has already been discussed, but the structural screening technique introduces new concepts that are worth further discussion. The structural controllability analysis is applied to a linear process model of the form:

$$
\begin{aligned}
& \dot{x}=A x+B u \\
& y=C x
\end{aligned}
$$

where $x, u$ and $y$ are the vectors of states, manipulated variables and control objectives. The procedure then assumes that the regulation of the objectives can be achieved using proportional-integral controllers (although it can be extended readily to consider more complex controllers), leading to a closed loop model of the form:

$$
\left[\begin{array}{c}
\dot{x} \\
\dot{z}
\end{array}\right]=\left[\begin{array}{ll}
A & 0 \\
C & 0
\end{array}\right]\left[\begin{array}{l}
x \\
z
\end{array}\right]+\left[\begin{array}{l}
B \\
0
\end{array}\right] u
$$

where $z$ is the vector of controller states. It is then shown that the system 3.1 cannot be controlled with any multiloop PI controller if the system 3.1 is not pointwise state controllable. Pointwise state controllability is defined as follows: The linear system

$$
\dot{x}=A(t) x(t)+B(t) u(t)
$$

where $x$ is the state vector and $u$ is the manipulated vector, is said to be completely controllable if the state of the system can be transferred from the zero state at any initial time, $t_{0}$ to any terminal state $x\left(t_{1}\right)=x_{1}$ within a finite time $t_{1}-t_{0}$ through the use of a piecewise continuous control input $u(t)$ (Kalman, 1960). A necessary and sufficient condition for pointwise state controllability is that the augmented matrix:

$$
\left[B|A B| A^{2} B|\ldots| A^{n-1} B\right]
$$

is of rank $n$ where $n$ is the dimension of the state matrix $A$. Applying this condition to the system 3.1 , it is shown that 3.1 is controllable if the following conditions are satisfied:

1. The pair $(A, B)$ is controllable 
2. The rank of $\left[\begin{array}{cc}A & B \\ C & 0\end{array}\right]$ is $n+r(r$ is the dimension of the control objective vector)

These rank conditions may then be applied to all possible control structures to eliminate the infeasible structures. However, it is noted that this procedure requires a full dynamic model of the process and may fail for isolated values of the process parameters (i.e. local singularities). Therefore a structural approach is proposed that allows rapid screening of alternative structures without developing a detailed dynamic model. Using this approach the system 3.1 is represented as a structural model (section 3.1.3). This leads to the following conditions for the system 3.1 to be structurally controllable:

1. The structural pair $(A, B)$ is of generic rank $n$. This is also the condition for structural controllability of the pair $(A, B)$

2. The generic rank of the structural matrix $\left[\begin{array}{cc}A & B \\ C & 0\end{array}\right]$ is $n+r$

The generic rank of a structural matrix is the highest rank that can be achieved by the matrix for some realisation of its nonzero elements. For example the structural matrix

$$
\left[\begin{array}{ll}
X & 0 \\
X & X
\end{array}\right]
$$

is of generic rank 2, as its rank is 2 for any nonzero diagonal elements, whereas

$$
\left[\begin{array}{lll}
X & 0 & 0 \\
X & X & 0 \\
X & X & 0
\end{array}\right]
$$

is of generic 2, as no realisation of the nonzero elements can result in a rank greater than 2. Algorithms have been developed for the rapid assessment of generic rank (Davison, 1977).

Given the structural controllability conditions developed above, the potential control structures for the functional units are screened by carrying out a combinatorial analysis on the manipulated variables to generate all possible square structures. The structural controllability test is applied to each structure, and all 
functionally uncontrollable structures are rejected. An efficient algorithm for the analysis is presented. Note that structural controllability of the system 3.1 does not guarantee state controllability, as the system may be locally singular at the desired operating point, but it still provides a useful screening tool for the control structure selection. The analysis also identifies pure integrators, that require control to ensure process stability.

The main contributions of this approach to regulatory control system synthesis are:

- The use of a hierarchical decomposition of the disturbances to define regulatory and optimising control tasks

- The use of operating requirements and process economics to identify regulatory control objectives

- Decomposition of the plant wide regulatory control system design problem into control system synthesis for functional units

- The systematic generation and screening of control structures through structural analysis

However the method only considers generation of control structures for a fixed set of control objectives, rather than trading off decreased control against reduced control cost. It also relies on heuristics for the final reduction of the feasible structures, and therefore the structures are not demonstrably optimal in any sense.

\subsubsection{Other structural approaches}

Johnston et al. (Johnston et al., 1985a; Johnston et al., 1985b) extend the concepts of Morari et al. with an improved algorithm to generate feasible structures and consider the elimination of control objectives when a full rank control structure cannot be attained with the set of available manipulated variables. The procedure also generates specific SISO pairings of the manipulated and measured variables. It does not include methods for the ranking of the alternative control structures, other than recommending dynamic simulation.

Georgiou and Floudas $(1989 \mathrm{a} ; 1989 \mathrm{~b} ; 1990 ; 1992)$ present an easily automatable generic rank algorithm using a mixed integer linear program (MILP) 
approach that can be used to systematically identify excess control objectives and manipulated variables. The algorithm can readily generate all structurally feasible control structures of a given rank by modifying a single parameter. This may be used to generate the set of all structurally feasible square control structures by varying the rank parameter from 1 to $n$, where $n$ is the minimum of $\{$ the number of control objectives, the number of manipulated variables $\}$. This procedure will generate all feasible control structures of dimensions $1 \times 1$ up to $n \times n$. Application of the algorithm to determine structural functional controllability (Russell and Perkins, 1987) is discussed, where structural functional controllability is the structural analogue of functional controllability - see section 3.2 for more detail. However, no ranking technique is proposed to choose between the structurally feasible alternative control structures generated by this procedure.

\subsubsection{Robustness based structure selection}

Reeves et al. (1991) propose the use of robustness properties as a screening tool for control structure selection. In this procedure, the design engineer specifies the expected model uncertainty as a relative additive uncertainty on the process transfer function between the possible manipulated and measured variables, where model uncertainty is used to describe mismatch between the process model and the actual plant. A screening algorithm is then applied to generate all square substructures capable of meeting the robustness requirement from the transfer function between the possible manipulated and measured variables. A stabilising feedback controller may be designed for any substructure that meets the robustness requirement. This procedure may be applied in a similar manner to that proposed for structural controllability to reduce the problem search space. The second stage of the procedure consists of an algorithm to choose decentralised controllers that minimise the degradation with respect to perfect control.

\subsubsection{Summary of control system synthesis techniques}

Consideration of the control structure selection problem posed in chapter 1 with respect to the synthesis techniques reviewed here reveals a number of deficiencies in the techniques, summarised as: 
- Aside from the unified approach of Stephanopoulos et al., the procedures either (i) generate very large numbers of alternative structures, or (ii) eliminate potential structures without performance evaluation, through decomposition techniques

- The relationship between control objectives and measured variables is not dealt with clearly

- The approaches to ranking structures are largely based on heuristics, rather than assessment of expected controller performance

- Process economics are used only to define the control objectives, and not for the examination of regulatory control structure performance

Two aspects that come out of the review are the desirability of including a screening analysis in the control structure selection problem to reduce the search space, and the need to develop a control structure ranking technique that can be used simultaneously with the generation of alternative control structures. Ideally, the ranking technique would be able to guide the generation of structures so that only "good" structures are chosen, thus reducing the combinatorial problem. If the ranking technique included the generation of a continuous performance index, this would suggest the use of mixed integer programming techniques, using integer variables to choose the control structure along with continuous optimisation of the performance index.

The next two sections discuss the recent approaches to control structure performance assessment (ranking techniques), and the assessment of the effects of control structure on process economics.

\subsection{Controllability analysis}

Controllability analysis is concerned with the dynamic characteristics of processes in the neighbourhood of a steady state operating point. Perkins (1989) contains a review of controllability, which breaks controllability analysis into three separate stages: Assessment; Diagnosis; and Design. Assessment is the calculation of a figure of merit for controllability to rank alternatives and highlight shortcomings. Diagnosis is used to explain any of the shortcomings in the analysis phase, and 
design is used to modify the process to improve the figure of merit and/or remove shortcomings. A good deal of research on the assessment of controllability has been carried out and is reviewed briefly below, but little work has been carried out on the diagnosis and design problems.

Most controllability assessment has been concerned with the attainability of perfect plant control, limited by factors that prevent physically realisable inversions of the plant transfer function, such as time delays, right half plane zeros, model uncertainty and manipulated variable constraints. The two approaches along these lines are: dynamic resiliency as proposed by Morari et al. (Morari, 1983; Grossmann and Morari, 1984; Palazoglu and Arkun, 1985; Palazoglu and Arkun, 1986) and functional controllability as proposed by Rosenbrock (1970) (Perkins and Wong, 1985; Russell and Perkins, 1987; Perkins, 1989; Barton et al., 1991).

Dynamic resilience (Morari, 1983) uses the internal model control (IMC) structure to represent the best possible controller achievable. For a perfectly controlled process, the controller must be the inverse of the process transfer function. However the physical realisability of such a controller is limited, and dynamic resilience may be used to generate an index that measures the attainability of perfect control (or the limits on the physical realisation of the perfect controller).

To generate the dynamic resilience index, the model of the process transfer function $\tilde{G}$ is decomposed into an invertible matrix $\tilde{G}_{-}$and a noninvertible matrix $\tilde{G}_{+}$(i.e. $\tilde{G}=\tilde{G}_{+} \tilde{G}_{-}$), where $\tilde{G}_{+}$contains all nonminimum phase elements (time delays and right half plane zeros) that prevent realisable system inversion. The controller transfer function is then taken as the inverse of $\tilde{G}_{-}\left(G_{c}=\tilde{G}_{-}^{-1}\right)$. Using this approach, the size of the admissible difference between the actual plant and the mathematical model used in the controller may be characterised in terms of robustness indices, that may be computed from the plant model alone (Palazoglu and Arkun, 1986). Thus, the relative magnitudes of these indices for different plant options may be used to rank the options with respect to their inherent controllability.

Functional controllability (Rosenbrock, 1970) is defined by considering a linear state space system of the form:

$$
\begin{aligned}
\dot{x} & =A x+B u(t)+E p(t) \\
y(t) & =C x+D u(t)+F p(t)
\end{aligned}
$$


where $x$ is the vector of state variables, $u$ is the vector of manipulated variables, $p$ is the vector of disturbance variables and $y$ is the vector of output variables.

The above system is called functionally controllable if given any $\{y(t)\}$ that is zero for $t<0$ and that is sufficiently smooth for $t \geq 0$, there exists a $\{u(t): t>0\}$ such that with $x(0)=0,\{u(t)\}$ generates $\{y(t)\}$.

This definition may be applied to both the servo and regulatory control cases with a little manipulation (Perkins, 1989). It states that for a plant to be controllable, there must exist a $u(t)$ that can generate any $y(t)$ that is desired. It can be shown that the linear system must be invertible to be functionally controllable. However, as noted previously, several process attributes result in physically unrealisable inverses. Therefore, functional controllability analysis considers the effects that prevent this physical attainability.

Rosenbrock (1970) considered the effects of RHP zeros and time delays on functional controllability. Perkins and Wong (1985) have characterised the effect of time delays with a parameter $\Delta_{\min }$, the minimum time before a trajectory for any $y(t)$ can be specified. $\Delta_{\min }$ is calculated based on the magnitudes and locations of delays in the process transfer function.

The Singular Value Decomposition (SVD) analysis of the transfer function $G$ can be used to characterise the effects of manipulated variable constraints for multivariable systems (MacFarlane and Jones, 1979). The SVD decomposition of $G(j \omega)$ results in:

$$
y(j \omega)=U \sum V^{H} u
$$

where $U$ and $V$ are unitary orthonormal matrices made up of the right and left singular vectors of $G(j \omega), \sum$ is a diagonal matrix of the singular values of $G(j \omega)$ and $u$ is the vector of manipulated variables. It can be shown that:

$$
\sigma_{\min }\left\|u_{\max }\right\|_{2} \leq\|y(j \omega)\|_{2} \leq \sigma_{\max }\left\|u_{\max }\right\|_{2}
$$

where $\sigma_{\max }\left(\sigma_{\min }\right)$ is the maximum (minimum) singular value of $G(j \omega),\|.\|_{2}$ is the two norm of the vector and $u_{\max }$ is the vector of manipulated variable bounds. Thus the larger the singular values, the larger the output region for which trajectories can be specified. Perkins notes that the process condition number $\gamma$ is another useful measure of the effects of manipulated variable constraints:

$$
\gamma=\frac{\sigma_{\max }}{\sigma_{\min }}
$$


If $\gamma$ is large, the outputs will show a strong directionality based on the inputs, and therefore limit the specification of trajectories. Thus for good control when the plant has manipulated variable constraints, the following is required:

$$
\begin{gathered}
\sigma_{\min }(\omega) \text { LARGE } \\
\gamma(\omega) \text { SMALL }
\end{gathered}
$$

Perkins and Wong (1985) point out that the condition number can also be used to characterise the effects of model uncertainty. For a given mismatch between the process model and the actual process (as an uncertainty region around the model), the larger the process condition number, the larger the uncertainty in input trajectory required to achieve the desired output trajectory. Thus it is also desirable to have small condition numbers to reduce the effect of model uncertainty. Note that the SVD is highly scale dependent, and care must be taken when comparing different systems. The scaling used for condition number calculations depends on whether it is being used for manipulated constraint assessment or for uncertainty assessment. Johnston and Barton (1987) and Skogestad and Morari (1987) recommend scalings for manipulated constraint analysis. Perkins and Wong (1985) and Morari and Skogestad (1985) suggest scalings to minimise the condition number for robustness analysis.

Thus consideration of functional controllability leads to three separate indices:

$$
\Delta_{\min }, \sigma_{\min }(\omega) \text { and } \gamma(\omega)
$$

These three indices (four if two scalings of $\gamma$ are used) may be used to characterise plant behaviour that limits the attainability of perfect control. However, there is no obvious relationship between these parameters, and it is not obvious which parameter should be improved while penalising the others. This is particularly the case for the condition numbers and singular values, where the only quantification of values is small and large.

Both the functional controllability and dynamic resilience approaches may be used to rank different processes with similar economics (i.e. choose the process with the better robustness), but are not as useful for comparing processes with differing economics and robustness indices, as the value of an improved robustness index is not clear. This is because the controllability indicators generate indices 
of the quality of control attainable for the selected control structure, rather than indicating the performance of the control structure with respect to the process control objectives. An approach to avoid this problem is the use of multi-objective optimisation (pareto-optimal or non-inferior set) techniques to generate trade off curves between steady state economics and controllability indicators (Palazoglu and Arkun, 1986; Palazoglu and Arkun, 1987; Luyben and Floudas, 1992), where any improvement in one objective results in a worsening of the other objective. However this approach still does not address the issue of the value of the improvement in robustness indices.

From this it can be seen that it would be desirable to have an index of performance that allows estimation of the value of control. Ideally, from the design engineer's point of view, this index would be an economic performance indicator, as this would allow a direct trade off with the steady state economics (i.e. an evaluation of the dynamic economics). The following section addresses existing approaches to assess dynamic economics.

\subsection{Economic analysis of dynamics}

As has been discussed at some length, to achieve optimal operation it is often desirable to operate a process on some of its constraints. In practice, it will not actually be possible to operate on these constraints, as process disturbances will often cause the plant to violate the constraints. Thus it is necessary to move the steady state operating point sufficiently far into the feasible region to ensure that no constraint violation occurs during plant operation. It can be seen that process control considering economic performance tries to minimise the process variability close to the active constraints (the less the dynamic variability, the closer the plant can be operated to the constraint). This concept of active constraint back off is used in control retrofit studies (Tyrrell, 1983; Marlin et al., 1991), where the improvement in performance achieved by upgrading the control system is estimated by the reduction in required constraint back off due to reduced variability in the constraint slacks.

Using the concept of constraint back off, the economic effect of disturbances may be measured conveniently by examining their impact on the variables in the 
model that represent the violation of active constraints at the steady state optimum (the active constraint slack variables). If under normal operation, the value of the slack variable of such a constraint takes a nonzero value, then that constraint will be violated. To remove the constraint violation, the operating point must be moved a sufficient distance into the operating region to ensure the constraint is no longer violated. The process economics at the new operating point are the dynamic economics of the process.

Formalising these concepts, if it is possible to estimate the back off from the active process constraints, then the dynamic economics may be estimated from the steady state process optimum described by:

$$
\begin{array}{r}
\min _{d} J_{0}(d) \\
h(d) \leq 0
\end{array}
$$

with associated first order optimality conditions (Reklaitis et al., 1983):

$$
\begin{aligned}
\nabla J_{0}(d)+\sum_{i=1}^{I} \lambda_{i} \nabla h_{i}(d)= & \\
h(d) & \leq 0 \\
\lambda_{i} h_{i}(d) & =0 \\
\lambda & \geq 0
\end{aligned}
$$

Where $d$ are the process parameters at the steady state optimum, $J_{0}$ is the objective function and $h$ is the vector of process constraints. $\lambda$ is the vector of Lagrange multipliers associated with $h$, where the $\lambda$ are a first order estimate of the sensitivity of the objective function to movement in the associated slack variable.

If a measure of the dynamic back off from each active constraint is available, then using the steady state optimum economics and active constraint Lagrange multipliers, a first order estimate of the dynamic economics is given by:

$$
J=J_{0}+\sum_{i=1}^{n} \lambda_{i} \delta_{i}
$$

where $J$ is the objective function at the actual point of operation (dynamic economics), $J_{0}$ is the steady state nonlinear optimum objective function, $\lambda_{i}$ is the Lagrange multiplier of the $i$ th active constraint, $\delta_{i}$ is the back off required in the $i$ th constraint and $n$ is the number of active constraints. 
This approach for evaluating the dynamic economics requires an estimate of the required dynamic back off. Perkins (1989) proposed a calculation based on singular value analysis of the form:

$$
\hat{\delta}=\|\bar{\delta}(j \omega)\|_{2} \leq \sigma_{\max }\left(G_{p}(j \omega)\right)\|\bar{p}(\omega)\|_{2}
$$

where $\sigma_{\max }$ is the maximum singular value of the transfer function, $G_{p}$, between the process disturbances, $p$, and the active constraint slack variables, $\delta$, and $\hat{\delta}$ is the "average" back off required in each active constraint. This method suffers from significant scaling problems, as constraints with large slack variables will skew the normalised back off to high values, which can lead to very conservative estimates of dynamic economics if there are constraints which have small expected back offs and large Lagrange multipliers (e.g. composition constraints). That is, the normalisation can overemphasise the effects of some constraints. To avoid the problem associated with scaling of constraints, Gannavarapu et al. (Perkins et al., 1989; Gannavarapu, 1991) suggest an information rich approach to estimate the back off in each constraint separately. Starting with the open loop transfer functions between the active constraint slack variables and the process disturbances, $d$, and manipulated variables, $u$,

$$
\bar{\delta}=G(s) \bar{u}+G_{p} \bar{d}
$$

they note that an estimate of the open loop back off $(\bar{u}=0)$ for each constraint is given by:

$$
\delta_{i}(\omega)=\max _{j \in J}\left|G_{p}(\omega)_{i j}\right|\left|\bar{p}_{j}(\omega)\right| \forall i=1,2, \ldots, n
$$

That is, the expected back off in each constraint is given by the maximum over the possible disturbances of the gain and disturbance magnitude product. This assumes that the process is only subject to the action of a single disturbance at any point in time, and therefore only the largest disturbance effect need be considered for calculation of the back off. If the open loop dynamic economics are "close" to the steady state optimum process economics, then no control is recommended. Otherwise, the economically significant back offs are identified, and the closed loop back off for each economically significant constraint is assessed to generate the closed loop dynamic economics. Gannavarapu (1991) proposes heuristic estimation of the closed loop dynamic economics of the control structure by using the second largest constraint 
back off, and assessing the attainability of control using functional controllability indicators. It is noted that if perfect closed loop disturbance rejection can be achieved for this system, then the required back off in each constraint is zero, and the process may operate at its steady state optimum. Gannavarapu (1991) introduces a second heuristic to handle the case where a calculated back off will result in violation of another constraint (i.e. the back off requires the process to violate another constraint). In this case, the back off is truncated at the maximum feasible back off for calculation of the dynamic economics. This approach suffers from the following drawbacks:

- It is unlikely that the process would be subject to only one disturbance at any point in time, and therefore the open loop procedure is likely to underestimate the required back off

- The use of the maximum feasible back off heuristic indicates that the plant would be infeasible under some disturbance conditions, and therefore the cost is infinite as operation will be impossible under these disturbance conditions (assuming hard constraints)

- The closed loop back off heuristic does not have a theoretical basis, and therefore may be either extremely conservative or optimistic depending on the process and disturbances under consideration

An extension of this method would be to use a generalised controller tuning technique like BLT tuning (Luyben, 1990) or $H_{\infty}$ (Morari and Zafiriou, 1989), to generate an implementable control system. The process and controller could then be treated as an open loop system and an estimate of the closed dynamic economics could be calculated as above. However, this approach introduces the undesirable requirement of tuning a controller for the evaluation of each control structure's dynamic economics.

In spite of its drawbacks, this approach allows rapid estimation of dynamic economics and the ability to categorise constraints as having either minor or major economic impact, where constraints with major impact are controlled, and those with minor impact are operated open loop. That is, the approach allows assessment of the trade off between installed controller costs and the economic benefits of the 
controller. The work on control structure selection in this thesis will consider modifications to this approach to remove the disadvantages outlined above, and to develop the approach as a systematic method for the assessment of dynamic economics.

\subsection{Linear versus nonlinear dynamic analysis}

This section discusses of the relative benefits of linear and nonlinear dynamic analysis for the control structure selection problem. Given the general control structure selection problem formulated in chapter 2 , the obvious solution method is to solve the optimisation problem directly. However, this formulation is a mixed integer dynamic optimisation problem of high complexity with an infinite set of constraints and a semi-infinite set of inputs. As was noted in chapter 2, this form of problem would not be easy to solve. However by examining the literature, it can be seen that there is a great deal of information on dynamic analysis for linear systems. This suggests a second approach taking advantage of linear systems theory, using some form of combined linear/nonlinear analysis. The nonlinear analysis can be used for the steady state design (a well understood problem), and a linearised approximation of the nonlinear model can be used for the dynamic analysis. An outline of the advantages and disadvantages of each approach is given here.

Advantages of linear analysis techniques:

- The great majority of control system theory literature uses linear systems theory

- The mathematics associated with linear systems is simpler than that of nonlinear systems

- Use of linear systems theory allows some circumvention of the infinite problem constraints

Disadvantages of linear analysis techniques

- Analysis using linear approximations to the nonlinear equations does not guarantee that the results will be optimal

- As linearisation is only accurate within a "small" area around the point of optimisation, use of a linear analysis may not catch switching of active constraints due to consideration of plant dynamics 
- If frequency response theory is used to study the plant dynamics, any early peaks and troughs in the disturbance response are ignored (as frequency response theory considers the asymptotic behaviour of the process as time tends to infinity). In general for a stable system, these values will have larger magnitudes than those after the process has settled

Advantages of nonlinear analysis techniques:

- There is no loss of information, as the full problem representation is utilised

Disadvantages of nonlinear analysis techniques:

- The problem size is large and solution may prove intractable

- With a finite time for analysis it is impossible to satisfy either the infinite time horizon or the analysis of every possible disturbance combination. However as it has been stated that one of the problem objectives is to generate a closed loop stable system, it is not necessary for the analysis to examine an infinite time horizon, but rather a finite horizon when the process is regarded as settled. The main difficulty lies in determining the appropriate length of the time horizon

Both the MINLP and linear/nonlinear approaches will be investigated in this thesis, initially considering the linear/nonlinear case, as this may provide useful insights into the nonlinear case both in terms of formulation and solution methods. This approach is suggested by Papoulias and Grossmann (1983) and Duran and Grossmann (1986a), where methods for mixed linear programs for process synthesis were used to develop mixed integer nonlinear programming approaches for process synthesis.

\subsection{Summary}

Based on the review of material relevant to the control structure selection problem, the following conclusions were drawn:

- Both mixed integer nonlinear programming and linear/nonlinear dynamic approaches to the problem will be considered 
- Any approach developed should include systematic screening methods to reduce the search space

- Evaluation of control structure requires assessment of dynamic economics

- Any performance indicator developed for the assessment of control structures should be employable as a tool to guide the generation of alternative control structures (i.e. the performance analysis should also be able to act as a screening tool)

- Existing tools for the assessment of dynamic economics supply useful information about control objectives, but have significant disadvantages which should be overcome before they can be employed as systematic design tools 


\section{Chapter 4}

\section{Linear analysis of dynamic economics}

This chapter develops a linear analysis of the dynamic economics based on the ideas of Perkins et al. (1989). The proposed analysis assesses the impact of disturbances on plant economic performance as follows: Nonlinear steady-state optimisation is performed to determine the best operating point in the absence of disturbances. Frequency response analysis of the linearised plant dynamic model is used to estimate the effects of plant disturbances on this ideal performance under a variety of control strategies. The analysis is illustrated on a case study involving minerals flotation circuit design, and it is shown to be suitable for use during process design for the evaluation of alternative process and control structures.

\subsection{Linear dynamic economics}

In the previous chapter, we introduced the concept of dynamic economics based on a required back off from the active constraints:

$$
J=J_{0}+\sum_{i=1}^{n} \lambda_{i} \delta_{i}
$$

where $J_{0}$ is the steady state minimum objective function, $J$ is the dynamic economics of the process, $\delta_{i}$ is the required back off from the $i$ th constraint, $\lambda_{i}$ is the Lagrange multiplier of the $i$ th slack variable, and $n$ is the number of active constraints. The calculation suggested by Perkins et al. (1989) suffers from the disadvantage that it can significantly underestimate the back off, as it assumes that the process will only ever be subject to one disturbance at any given time (this allows calculation of the back off as the largest single disturbance effect on each active constraint). If the back off is underestimated, then it is likely that the process will violate the active constraints during operation, leading to infeasible operation. This suggests that a better approach to the problem would be to estimate the maximum expected back off in each constraint, as this would ensure the process could be operated without violating its constraints, and thus satisfy the hard constraint condition imposed on 
the control structure selection problem in chapter 1 . That is, the dynamic economics are calculated as:

$$
J=J_{0}+\sum_{i=1}^{n} \lambda_{i} \max \delta_{i}
$$

where $\max \delta_{i}$ is the maximum required constraint back off.

One approach to estimating $\max \delta_{i}$ is to apply frequency response theory to examine the behaviour of the active constraint slack variables, $\bar{\sigma}(s)$. These variables are given by:

$$
\bar{\sigma}(s)=G(s) \bar{p}(s)
$$

where $G(s)$ is a linear transfer function and $\bar{p}$ is the $m$-vector of process disturbances. Assuming the disturbances are sinusoids of the form $p_{l}(t)=\left|p_{l}\right| \sin \left(\omega t+\phi_{l}\right)$, where $\left|p_{l}\right|$ is the disturbance amplitude, $\omega$ is the disturbance frequency and $\phi$ is the disturbance phase lag and applying frequency response theory (Stephanopoulos, 1984), the ultimate response of slack variable $i$ to sinusoidal disturbance $l$ is given by:

$$
\lim _{t \rightarrow \infty}\left|G_{i l}(j \omega)\right|\left|p_{l}\right| \sin \left(\omega t+\phi_{l}+\theta_{i l}\right)
$$

where $\theta_{i l}$ is the argument of the $i$ th element of the transfer function. Applying this result to the response of slack variable $i$ to all of the disturbances results in:

$$
\sigma_{i}(t)=\sum_{l=1}^{m}\left|G_{i l}(j \omega)\right|\left|p_{l}\right| \sin \left(\omega t+\phi_{l}+\theta_{i l}\right)
$$

The maximum amplitude of $\sigma_{i}$ with respect to the disturbance phase lags is an estimate of $\max \delta_{i}$ :

$$
\tilde{\sigma}_{i}=\max _{\phi}\left|\sigma_{i}\right|=\max _{\phi}\left|\sum_{l=1}^{m}\right| G_{i l}(j \omega)|| p_{l}\left|\sin \left(\omega t+\phi_{l}+\theta_{i l}\right)\right|
$$

where $\tilde{\sigma}_{i}$ is the maximum slack variable amplitude of oscillation. Note that $\tilde{\sigma}_{i}$ is an approximation to $\max \delta_{i}$ as frequency response theory considers only the asymptotic plant behaviour, and ignores initial transients (these are due to the complementary solution of the process response (Luyben, 1990)). By inspection, the maximum occurs when $\phi_{l}=-\theta_{i l}$, as this allows direct summation of the amplitudes of the contributing disturbances, resulting in:

$$
\tilde{\sigma}_{i}=\max _{\phi}\left|\sigma_{i}\right|
$$




$$
\begin{aligned}
& =\left|\sum_{l=1}^{m}\right| G_{i l}(j \omega)|| p_{l}|\sin (\omega t)| \\
& =\sum_{l=1}^{m}\left|G_{i l}(j \omega)\right|\left|p_{l}\right|
\end{aligned}
$$

Given the transfer function $G$, this back off can be readily calculated.

The next step in the proposed dynamic economic analysis is the generation of $G(s)$. This may be developed by considering a variant of the linear DAE process model suggested by Perkins et al. (1989):

$$
\begin{aligned}
\dot{x} & =A x+B z+C u+D p \\
0 & =E x+F z+G u+H p \\
y & =Q\left[\begin{array}{l}
x \\
z
\end{array}\right]
\end{aligned}
$$

where $x, u, z, p$ and $y$ are the vectors of state, manipulated, algebraic, disturbance and measurement variables respectively. $Q$ is a permutation matrix that selects the variables which are to be measured for control purposes from the vectors $x$ and $z$. It is a sparse matrix, with one unity element in each row to select the measured $x$ and $z$. It is assumed that the dimensions of $y$ and $u$ are equal (i.e. a square control structure), and the slack variables of the active constraints are included in the vector of algebraic (output) variables. To determine the constraint back off requires the transfer function between $z$ and the disturbance $p$.

Following the ideas of Perkins et al., initially consider the open loop back off (i.e. no control). Taking Laplace transforms leads to:

$$
\begin{aligned}
(s I-A) \bar{x}-B \bar{z} & =C \bar{u}+D \bar{p} \\
-E \bar{x}-F \bar{z} & =G \bar{u}+H \bar{p}
\end{aligned}
$$

where $\bar{u}$ and $\bar{p}$ are the process inputs. If the process is operated open loop subject to a disturbance of amplitude $p(\omega)$, then $\bar{u}=0$ and $G(j w)$ is given by:

$$
\left[\begin{array}{c}
\bar{x} \\
\bar{z}
\end{array}\right]=\left[\begin{array}{cc}
j \omega I-A & -B \\
-E & -F
\end{array}\right]^{-1}\left[\begin{array}{l}
D \\
H
\end{array}\right] p(\omega)
$$

which allows calculation of $\tilde{\sigma}$ ( $\sigma$ is a subset of $z$ ), and hence an estimate of the dynamic economics using equation 4.1 where $\max \delta$ is set to $\tilde{\sigma}$. 
However, we desire an estimate of the closed loop economics to evaluate the expected performance of the control structure under examination. One possible approach would be to tune a process controller of the form $\bar{u}=K(s) \bar{y}$, and by substituting for $\bar{u}$, generate the closed loop transfer function between $\bar{p}$ and $\bar{\sigma}$, and then evaluate the closed loop dynamic economics as above. However, tuning a controller for each potential control structure would prove extremely time consuming. Therefore an alternative approach is developed from functional controllability concepts, where we consider perfect control of the process measurements. That is, $\bar{u}$ is varied to achieve

$$
\bar{y}=Q\left[\begin{array}{l}
\bar{x} \\
\bar{z}
\end{array}\right]=0
$$

This leads to the perfectly controlled Laplace transform:

$$
\begin{aligned}
(s I-A) \bar{x}-B \bar{z}-C \bar{u} & =D \bar{p} \\
-E \bar{x}+-F \bar{z}-G \bar{u} & =H \bar{p} \\
Q\left[\begin{array}{c}
\bar{x} \\
\bar{z}
\end{array}\right] & =0
\end{aligned}
$$

and a perfect control transfer function:

$$
\left[\begin{array}{c}
\bar{x} \\
\bar{z} \\
\bar{u}
\end{array}\right]=\left[\begin{array}{ccc}
j \omega I-A & -B & -C \\
-E & -F & -G \\
Q_{x} & Q_{z} & 0
\end{array}\right]^{-1}\left[\begin{array}{c}
D \\
H \\
0
\end{array}\right] \bar{p}(\omega)
$$

where $Q_{x}$ and $Q_{z}$ are appropriate partitions of $Q$. The perfect control back off and corresponding dynamic economics may then be calculated in the same fashion as the open loop case. The perfect control economics are an indicator of the best attainable economics for the given control structure (measurements and manipulated variables). Note that this analysis does not require that the constraint slack variables are measured, but only that they are included in the algebraic variable vector, $z$. This implies that the dynamic economics for any set of measured and manipulated variables can be rapidly assessed. As the perfect control assumption is being used, a functional controllability analysis is also necessary to check the attainability of perfect control (section 3.2).

Extending these ideas to nonlinear models, a linearised model of a nonlinear process can be used to calculate max $\delta$ provided the following assumptions hold: 
1. The process is not highly nonlinear

2. There are no inactive constraints in the immediate region of the optimum

3. The optimum lies on a set of convex constraints

Assumption 1 is required to ensure the validity of the linearised model. If assumption 2 is not met, the process may dynamically encounter a previously inactive constraint and thus invalidate the analysis. Assumption 3 ensures that the dynamic operating point will lie close to the steady state optimum.

Thus to apply the above method to a nonlinear system, the following algorithm is used:

1. Perform a steady state nonlinear optimisation of the process.

2. Linearise the process at the optimum, choosing the active constraint slack variables as outputs, along with any other controlled variables

3. Calculate $\tilde{\sigma}$.

4. The optimum dynamic operating point is then at a distance of $\tilde{\sigma}$ from the steady state active constraints, and the operating cost for a minimisation problem is given by:

$$
J=J_{0}+\sum_{i=1}^{n} \lambda_{i} \tilde{\sigma}_{i}
$$

where $\lambda_{i}$ is the steady state Lagrange multiplier of constraint $i$

The following section contains a case study demonstrating the application of the method.

\subsection{Case study - Froth flotation circuits}

The case study is based on that used by Barton et al. (1991). The aim of the original study was to select an optimal, controllable flowsheet for a mineral flotation circuit. The problem has been re-evaluated using the dynamic economic analysis outlined in the previous section.

A flotation circuit separates valuable minerals from gangue material via preferential flotation of the valuables. Separation is carried out in a flotation cell 
(shown in figure 4.1), where a slurry of gangue and valuable is pumped into an air sparged tank. The hydrophobic valuable material preferentially enters the froth phase and exits from the top of the tank, while the gangue rich stream exits from the bottom. A flotation bank is made up by connecting a number of cells in series, and a flotation circuit is made by connecting flotation banks (not necessarily in series).

The Barton et al. case study considered flotation circuits made up of three flotation banks of one cell each (for simplicity of calculation). When the permutations of the connections of three cells are considered, 49 possible flowsheets can be drawn. Barton et al. considered 12 flowsheets in detail, and for this study, only the four flowsheets with the highest valuables recovery were examined (figure 4.2 - note the convention of drawing the concentrate from the bottom of the flotation banks).

To describe the behaviour of the flotation cells, Barton et al. modelled the cell as a well mixed tank, with the flotation mechanism described by first order kinetics, and the effect of the aeration modelled by an empirical factor affecting the kinetics. The equations describing this cell model are based on the work of Lynch et al. (1981), using a three component (water, valuables and gangue) model. The process model is summarised in appendix $A$.

After deciding on the process model, Barton et al. simulated the process using the SPEEDUP process simulation package and carried out a design optimisation to maximise valuables recovery by varying the cell volumes. A constraint was placed on the design by specifying a maximum total volume for the cells. A minimum product grade (dry product purity) was also specified.

In this study we expand on the previous work to utilise the dynamic economic analysis proposed in section 4.1. Having obtained the optimum design volumes for the cells, a second, operating optimisation was carried out, with the cell volumes at the design optimum. This two stage optimisation approach was used so that the results could be directly compared to those of Barton et al.. Normally a design study would be carried out using manipulated and design variables in a single optimisation. For the second optimisation, the recovery was maximised with respect to the manipulated variables (adding constraints in the form of upper and lower bounds on the manipulated variables in addition to the minimum product grade). The analysis of dynamic economics was then carried out using a linearised 


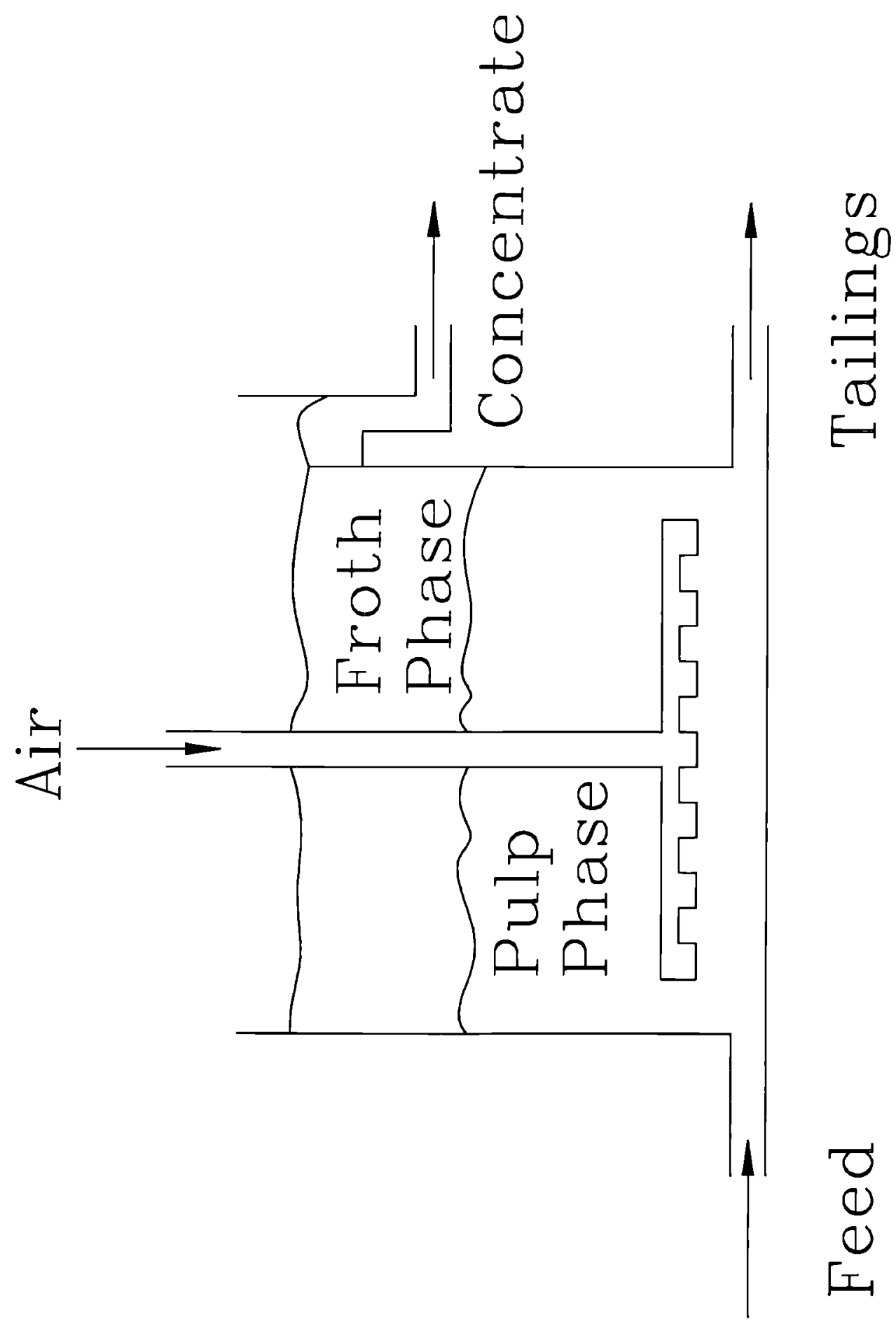

Figure 4.1: Froth flotation cell 

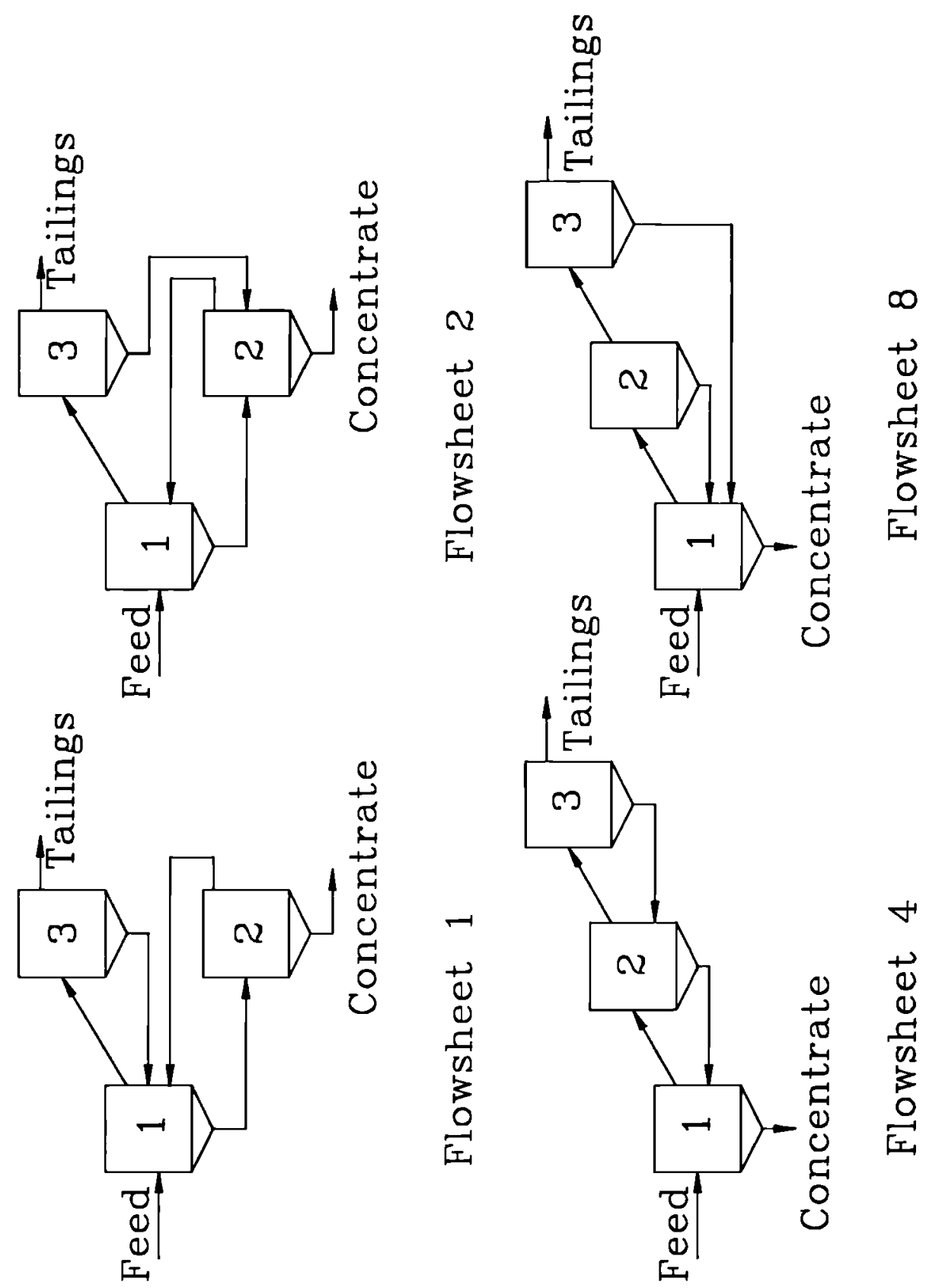

Figure 4.2: Froth flotation circuit flowsheets 
model of the process at the steady state operating optimum.

The process was optimised and linearised at the steady state optimum using SPEEDUP. The inputs for this problem were the feed water and control air and the outputs were the slack variables of the active constraints and the process recovery.

For this case study, the objective function is the maximisation of valuables recovery. As the active constraints for all flowsheets are minimum product grade and minimum percent water in the feed, an estimate of the average recovery in operation is:

$$
\begin{gathered}
\text { Operating recovery }=\mathrm{J} \\
=\text { Optimum steady-state operating recovery } \\
-\lambda_{\text {water }} \tilde{\sigma}_{\text {water }}-\lambda_{\text {grade }} \tilde{\sigma}_{\text {grade }}
\end{gathered}
$$

where $\lambda$ is the Lagrange multiplier and $\tilde{\sigma}$ is the maximum constraint back off. Note that maximisation results in subtracting the giveaway effects, rather than adding as is the case for minimisation.

We now consider the choice of control objectives. In the original case study, the control objectives were the product grade and the product recovery, implying a desire to maintain a tight control on the variation in recovery for downstream processing. However, as the operating constraints should not be violated, another obvious choice of control objectives are the slack variables associated with the active constraints (recovery and grade can both be measured using $\mathrm{X}$-ray fluorescence techniques (Fewings et al., 1979)). In this case it is assumed that the variation in recovery is irrelevant, as long as the average recovery is maintained at the highest possible level, by operating as close to the optimum constraints as possible. These two approaches will be referred to as recovery control and constraint control respectively.

Table 4.1 shows the results of the initial steady-state optimisation to determine the best distribution of volume among the three cells. The operating optimisation did not change the optimum recovery. This is because for all four cases, the optimum design conditions correspond to operation on the minimum feed water and minimum product grade constraints, which are also the active constraints for the operating optimisation. 


\begin{tabular}{|c|r|r|r|c|}
\hline Flowsheet & \multicolumn{3}{|c|}{ Cell Volumes $\left(\mathrm{m}^{3}\right)$} & Optimum Design \\
& \multicolumn{1}{|c|}{1} & \multicolumn{1}{c|}{2} & \multicolumn{1}{c|}{3} & Recovery (\%) \\
\hline 1 & 26.612 & 8.626 & 18.762 & 93.26 \\
2 & 25.334 & 8.662 & 20.004 & 93.65 \\
4 & 8.774 & 25.690 & 19.537 & 94.87 \\
8 & 8.785 & 25.183 & 20.033 & 94.99 \\
\hline
\end{tabular}

Table 4.1: Results of steady-state optimisation of flotation circuits

We now examine the effects of the disturbances on the plant performance. The disturbances were taken to be a $10 \%$ variation in both of the solid feeds (valuable and gangue) to the process. The frequency range for the controllability analysis was chosen to cover $\tau / 10$ to $10 \tau$, where $\tau$ is an estimate of the process time constant. This is based on the experience of Gannavarapu (1991), whose results indicate that most significant effects on the dynamic economics occur at a disturbance frequency near the dominant time constant of the process. For the flotation case study, $\tau$ was typically of the order 30 minutes, giving a frequency range of $1 / 300$ to $1 / 3$ cycles/minute. For convenience a log scale range of .001 to 1 cycle/minute was used. Figures 4.3-4.6 are graphs showing the relevant results of the dynamic economic analysis over the frequency range of interest. The $l_{\infty}$ minimised condition number (appendix B) was used to assess the attainability of perfect control as it supplies an upper bound on the two norm minimum condition number and its usefulness has been demonstrated in several case studies (Perkins and Wong, 1985; Barton et al., 1991; Gannavarapu, 1991). The results for $\omega_{\text {disturbance }}=1 / 30 \mathrm{cycles} / \mathrm{min}$ are summarised in table 4.2 , as these are representative of the plant behaviour over the frequency range.

From table 4.2, the following observations may be made: 1) Any given flowsheet under perfect constraint control yields a better recovery than the same flowsheet under perfect recovery control; 2) Flowsheet 8 yields the highest recovery under both schemes; 3) Recovery control should not be used for flowsheets 1 and 2 as the perfect control recoveries are worse than the uncontrolled recoveries. Result 3 is caused by unfavourable interactions between the dual requirement that the feed water be used to satisfy the minimum feed water constraint and simultaneously used as a manipulated variable (i.e. the dynamics of the feed water must satisfy the 


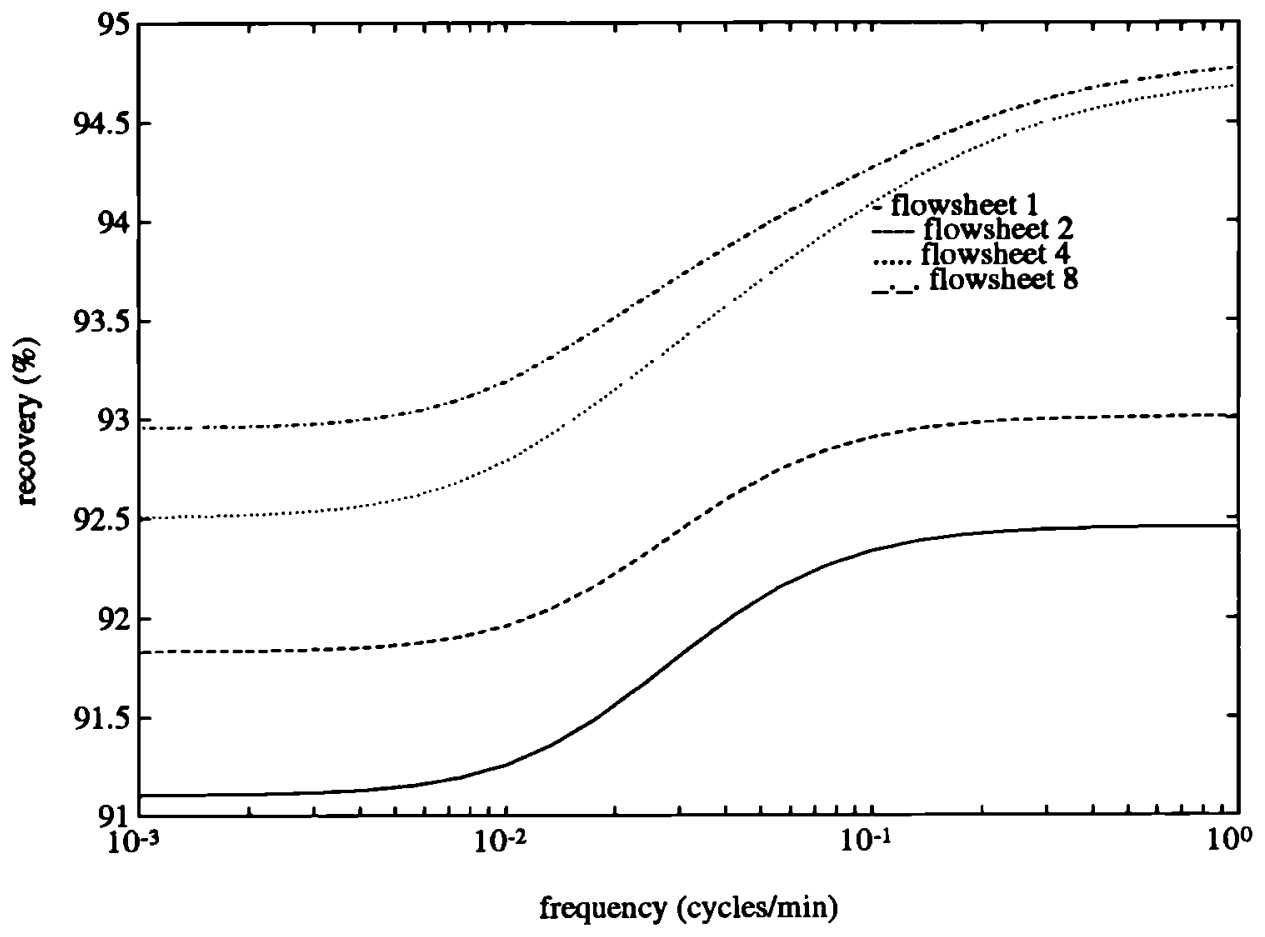

Figure 4.3: Process recoveries with no control

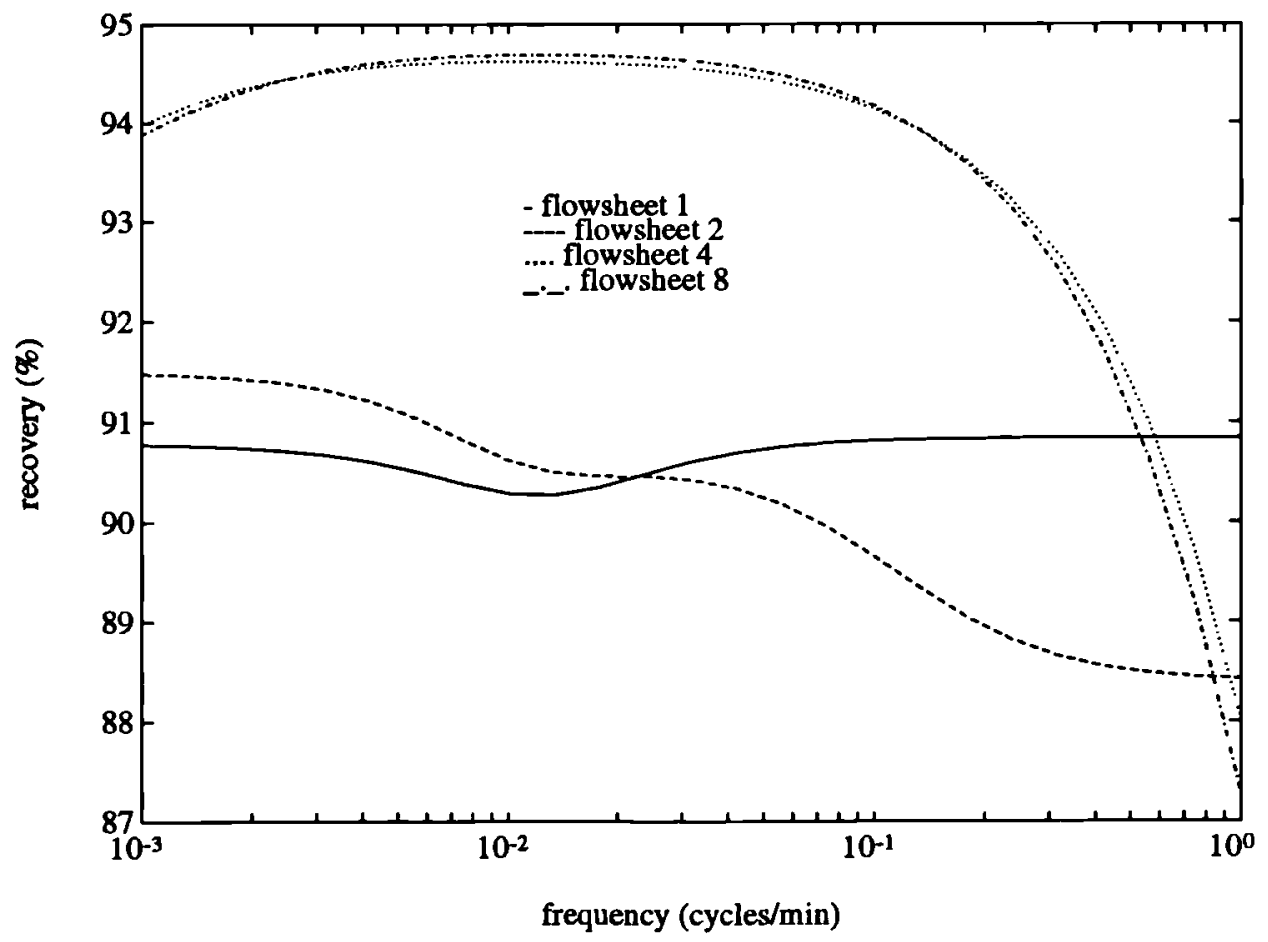

Figure 4.4: Perfect control recoveries (recovery control) 


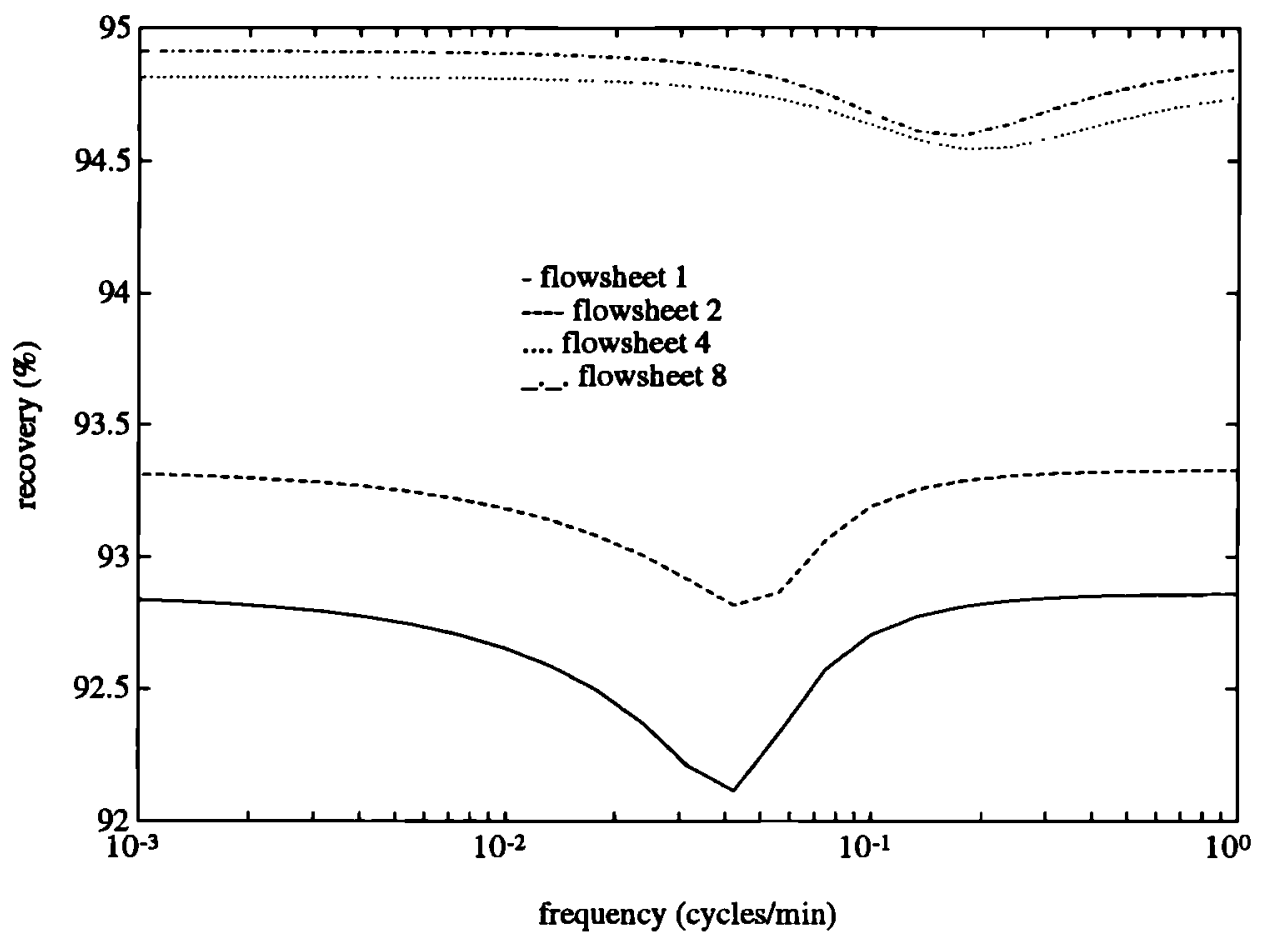

Figure 4.5: Process recoveries with BLT constraint control

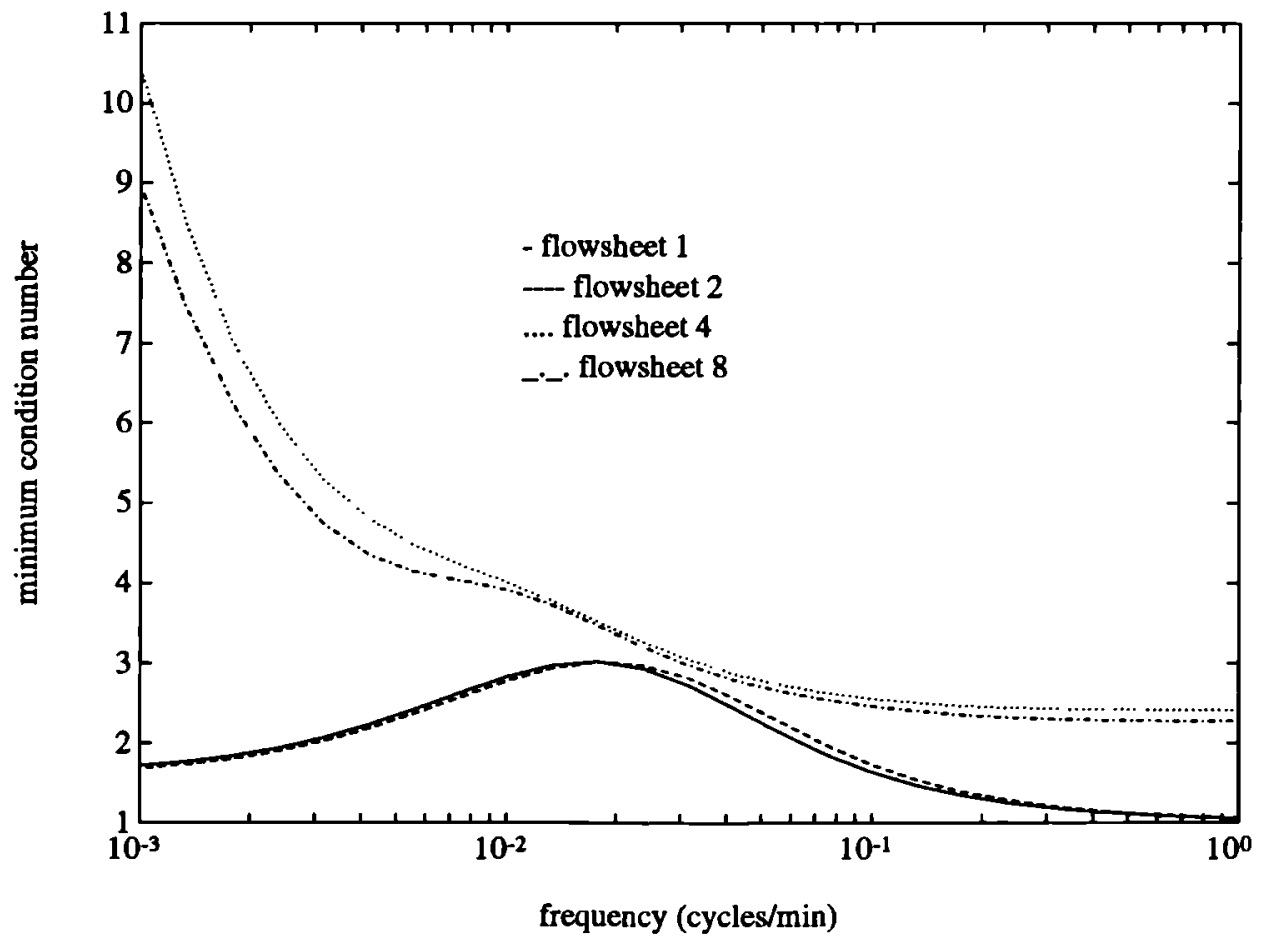

Figure 4.6: $l_{\infty}$ minimum condition number (recovery control) 


\begin{tabular}{|c|c|c|c|c|}
\hline \multicolumn{5}{|c|}{ Controllability analysis results: Recovery (\%) } \\
\hline \hline \multirow{2}{*}{ Flowsheet } & $\begin{array}{c}\text { Open } \\
\text { Loop }\end{array}$ & $\begin{array}{c}\text { Perfect } \\
\text { Constraint } \\
\text { Control }\end{array}$ & $\begin{array}{c}\text { Perfect } \\
\text { Recovery } \\
\text { Control }\end{array}$ & $\begin{array}{c}\text { BLT } \\
\text { Constraint } \\
\text { Control }\end{array}$ \\
\hline 1 & 91.88 & 93.26 & 90.62 & 92.18 \\
2 & 92.50 & 93.65 & 90.42 & 92.90 \\
4 & 93.46 & 94.87 & 94.54 & 94.78 \\
8 & 93.78 & 94.99 & 94.62 & 94.87 \\
\hline
\end{tabular}

Table 4.2: Average recovery for flotation circuits at disturbance frequency of $1 / 30$ cycles/min

\begin{tabular}{|c|c|c|}
\hline \multicolumn{3}{|c|}{$\begin{array}{c}\text { Controllability analysis results } \\
\text { Minimum Condition number }\end{array}$} \\
\hline \hline \multirow{3}{*}{ Flowsheet } & $\begin{array}{c}\text { Perfect } \\
\text { Constraint } \\
\text { Control }\end{array}$ & $\begin{array}{c}\text { Perfect } \\
\text { Recovery } \\
\text { Control }\end{array}$ \\
\hline 1 & 1 & 2.68 \\
2 & 1 & 2.78 \\
4 & 1 & 3.01 \\
8 & 1 & 2.93 \\
\hline
\end{tabular}

Table 4.3: $l_{\infty}$ minimum condition number at $w=1 / 30 \mathrm{cycles} / \mathrm{min}$

constraint at all times).

Having obtained the economics of perfect control, the $l_{\infty}$ norm minimum condition numbers are considered to assess the attainability of perfect control. Representative results are shown in table 4.3. The minimum condition numbers for the constraint control of all flowsheets equal one at all frequencies, which implies it will be relatively easy to achieve near perfect control. This is because the open loop transfer function for this configuration is lower triangular, and it can be shown that the minimum condition number for such a matrix will be unity (appendix B.1). The minimum condition numbers for recovery control are all higher, at roughly 2.8 . This means that it will be more difficult to control the processes using recovery control than using constraint control. From this it can be concluded that constraint control is the better method of control for all cases. Because all four flowsheets have the same minimum condition number under constraint control, no one flowsheet is eas- 
ier to control for these objectives than the others. The obvious choice is flowsheet 8 using constraint control, as it returns the highest recovery for the least control effort.

Given that flowsheet 8 under constraint control is chosen as the best configuration, the next step is to test whether it is economically viable to operate flowsheet 8 under control. To test this, a multiloop strategy was implemented based on controlling the grade using the air feed and the percentage water in the feed stream using the feed water. The BLT method was used to tune the PI controllers adopted (Luyben, 1990). The BLT recovery of flowsheet 8 is $94.87 \%$ and the uncontrolled recovery is $93.78 \%$, leading to an increase in recovery of $1.09 \%$. Given the typical throughputs and product values in flotation plants, a $1 \%$ increase in recovery will result in sufficient profit to justify the installation of an appropriate control scheme.

When it was attempted to tune BLT controllers for recovery control, the air-grade, water-recovery pairings were eliminated for flowsheets 1 and 2 as they were shown to be integrally unstable by RGA analysis. It proved impossible to generate stable BLT controllers for any of the remaining pairings (air-grade, waterrecovery for flowsheets 4 and 8 and air-recovery, water-grade for all flowsheets). This does not mean a stabilising controller does not exist, but simply that the BLT procedure could not stabilise the processes. This again suggests that constraint control is the better choice, as a relatively simple controller can be implemented to operate the plant.

It is interesting to compare our conclusions with those of Barton et al. for the same system. Barton et al. considered only recovery control, and on that basis selected flowsheets 1,2 as better than 4,8 using arguments based on open loop indicators. However from the results of the economic analysis, it can be seen that this decision is misleading, as flowsheets 1,2 are more economic to operate with no control than with perfect recovery control. Thus, although condition numbers indicate that it will be somewhat easier to achieve perfect control of flowsheets 1,2 than 4,8 , the economics indicate that the employment of this control is actually undesirable. Examination of the recovery control economics also shows that the uncontrolled performances of flowsheets 4 and 8 are better than both the uncontrolled and perfectly controlled flowsheets 1 and 2 . Thus by considering the process economics, the findings of Barton et al. are reversed, with flowsheets 4,8 preferable in spite of their 
higher condition numbers. Flowsheet 8 is the best choice of 4 and 8 as it has better economics and a similar condition number.

This result illustrates the problem with using open loop indicators as the sole measure of controllability, which is that if alternatives are ranked solely on that basis, flowsheets with good economic performance may be rejected unnecessarily.

\subsection{Summary}

This chapter developed an analysis based on conventional nonlinear steady state optimisation, linearised dynamics and functional controllability to study the dynamic economics of the process. The analysis was tested by examining a froth flotation circuit case study. The case study demonstrated that the combined dynamic/steady state economic analysis can be used to:

i) Select between alternative process flowsheets

ii) Select between alternative control configurations

It was also found that the combined analysis was more effective than a classical steady state economics/controllability analysis. The next chapter will consider extensions of this dynamic economic analysis as a potential approach to solving the control structure selection problem. 


\section{Chapter 5}

\section{Control structure selection based on linear analysis}

This chapter develops a technique for the solution of the control structure selection problem based on the linear dynamic economic analysis proposed in chapter 4 . The first section places the mathematics of the dynamic economic analysis on a more formal basis and develops a linear modelling framework suitable for control structure selection. The second section develops a hybrid mixed integer linear programming (MILP) description of the control structure selection problem, and discusses solution methods for the determination of the optimal structure. This chapter concentrates on the benefits obtained from use of linear analysis tools, and therefore any complex nonlinear problems which are developed during discussion are treated as dead ends and are not investigated further.

\subsection{General linear dynamic economic analysis}

This section considers extension of the dynamic economic analysis presented in the last chapter to handle inactive process constraints and the problem of infeasible constraint back off. Both problems were identified as flaws in the technique proposed by Gannavarapu et al. (1989), and neither were addressed in the previous chapter. Consider the following DAE model of a process plant with no controllers, based on the model developed in chapter 2:

$$
\begin{aligned}
& \min _{u_{\xi 0}, d_{\xi}} J_{\xi}\left(x_{\xi 0}, u_{\xi 0}, z_{\xi 0}, d_{\xi}, p_{\xi 0}\right) \\
\dot{x}_{\xi} & =f\left(x_{\xi}, u_{\xi}, z_{\xi}, d_{\xi}, p_{\xi}\right) \\
0 & =g\left(x_{\xi}, u_{\xi}, z_{\xi}, d, p_{\xi}\right) \\
0 & \geq h\left(x_{\xi}, u_{\xi}, z_{\xi}, d_{\xi}, p_{\xi}\right) \\
d_{\xi}^{\text {low }} & \leq d_{\xi} \leq d_{\xi}^{\text {high }} \\
u_{\xi}^{\text {low }} & \leq u_{\xi} \leq u_{\xi}^{\text {high }}
\end{aligned}
$$

where $x, u, z, d$ and $p$ are respectively the vectors of state variables, potential manipulated variables, potential measured variables (including all directly measurable 
states and slack variables), design variables and disturbance variables. The manipulated and design variables are subject to simple bounds. Subscript 0 indicates a steady state value and subscript $\xi$ a DAE variable. Note that $u$ and $d$ are time invariant parameters in the above formulation, and a distinction is only made between the two as the elements in $u$ may be varied in operation by a control system. The dynamic economics are to be assessed for the process subject to dynamic disturbances $p_{\xi}$. Assume that a nominal steady state optimum has been calculated for the disturbance $p_{\xi}(t=0)=p_{\xi 0}$, indicated by a superscript $*$ (i.e. optimisation with $\dot{x}_{\xi}=0$ ), and linearise the model at this steady state nonlinear optimum to yield the following linear deviation process model:

$$
\begin{aligned}
\min _{u_{0}, d} J & =\alpha^{T} x_{0}+\beta^{T} u_{0}+\gamma^{T} z_{0}+\delta^{T} d \\
\text { s.t. } \dot{x} & =A x+B u+C z+D d+E p \\
0 & =F x+G u+H z+K d+L p \\
a & \geq M x+N u+O z+P d+R p \\
d^{l} & \leq d \leq d^{h} \\
u^{l} & \leq u \leq u^{h}
\end{aligned}
$$

where $x, u, z, d$ and $p$ are the linear deviation variables given by $x=x_{\text {lin }}-x_{\xi 0}^{*}$ and so on, where $x_{\ln }$ is the linear approximation of $x_{\xi}$ obtained from Taylor series expansion of the DAE system. Similarly $J$ is the linear deviation from the nonlinear steady state objective function, given by $J=J_{\text {lin }}-J_{\xi}^{*}$. The vector of constants $a$ allows for linearisation of the nonlinear constraints $h$, and is given by:

$$
a=-h\left(x_{\xi 0}^{*}, u_{\xi 0}^{*}, z_{\xi 0}^{*}, d_{\xi 0}^{*}, p_{\xi 0}^{*}\right)
$$

from substitution of the Taylor series expansion of $h$ into the constraint $0 \geq h$.

The process disturbances $p$ either affect nominal inputs or steady state design variables. The variable affected is a design variable if it can be altered to improve the economics, such as the primary feed to a process, and it is a nominal variable if it is fixed by an upstream or external process, such as the feed to a separator from a reactor system. In either case, the value of steady state deviation is zero $\left(p_{0}=0\right)$, because:

- For the design variable case, all steady state effects are handled by the $d$ vector 
- For the nominal input case, the disturbance is a dynamic deviation from the steady state value. The steady state value of the nominal input is a fixed input imposed by the environment. Therefore, by definition $p_{0}=0$

This results in redundancy of information for the design variable case, as the coefficients of $d$ are also the coefficients of some elements of $p$, but this notation is used for convenience in the dynamic analysis.

Now consider the steady state linear program (LP) obtained by setting $\dot{x}=\mathbf{0}$.

$$
\begin{aligned}
\min _{u_{0}, d} J & =\alpha^{T} x_{0}+\beta^{T} u_{0}+\gamma^{T} z_{0}+\delta^{T} d \\
0 & =A x_{0}+B u_{0}+C z_{0}+D d \\
0 & =F x_{0}+G u_{0}+H z_{0}+K d \\
a & \geq M x_{0}+N u_{0}+O z_{0}+P d \\
d^{l} & \leq d \leq d^{h} \\
u^{l} & \leq u_{0} \leq u^{h}
\end{aligned}
$$

Viswanathan and Grossmann (1990) note that the optimum solution to this problem corresponds to the solution of the steady state nonlinear optimisation (i.e. the deviation objective function, $J$, is zero at the optimal solution of the LP), as the linearisation forms a convex hull at the point of linearisation. This has two effects when considering the dynamic economics:

1. As the dynamic effects can only further constrain the above problem (see chapter 2), an optimistic bound on the dynamic economics is given by the nonlinear steady state objective function. That is, it is not possible to predict dynamic economics better than the nonlinear steady state economics. This would not necessarily hold for a linearisation away from the optimum

2. If the dynamic effects are included directly in this model, the dynamic economics may be calculated as a giveaway from the nonlinear steady state objective function

The next step is to consider inclusion of the dynamic effects in the steady state model posed above. This was achieved in the last chapter by using a "safety margin" of the amplitude of the maximum slack variable oscillation of the active 
constraints, $\tilde{\sigma}$ (an approximation to the maximum constraint back off), to ensure that the plant would not violate constraints during normal operation. This may be included in the problem by adding $\tilde{\sigma}$ to the constraint equation.

$$
\begin{aligned}
\min _{u_{0}, d} J & =\alpha^{T} x_{0}+\beta^{T} u_{0}+\gamma^{T} z_{0}+\delta^{T} d \\
0 & =A x_{0}+B u_{0}+C z_{0}+D d \\
0 & =F x_{0}+G u_{0}+H z_{0}+K d \\
a & \geq M x_{0}+N u_{0}+O z_{0}+P d+I_{\sigma} \tilde{\sigma} \\
d^{l} & \leq d \leq d^{h} \\
u^{l} & \leq u_{0} \leq u^{h}
\end{aligned}
$$

Note that in this case, $\tilde{\sigma}$ is the maximum amplitude of all of the constraint slack variables in the problem, not just the active constraints. Assuming that $\tilde{\sigma}$ can be calculated, this formulation addresses the two failings identified in the Gannavarapu et al. (1989) approach:

- If the problem is infeasible, then it will be impossible to operate the plant without violating constraints dynamically, therefore either the design or the controller need to be modified

- If the problem is feasible, the solution takes all of the constraints into account directly, as all constraints and their variations are included in the problem

The above representation can account for simple bounds on the manipulated variables in normal operation by adding a set of constraints to the steady state LP to explicitly account for the dynamic variation of the manipulated variables:

$$
\begin{aligned}
& u^{l} \leq u_{0}-U \leq u^{h} \\
& u^{l} \leq u_{0}+U \leq u^{h}
\end{aligned}
$$

where $U$ is an estimate of the maximum dynamic variation in $u$, and is obtained in a similar manner to $\tilde{\sigma}$.

This leads to the steady state LP:

$$
\begin{aligned}
\min _{u_{0}, d} J & =\alpha^{T} x_{0}+\beta^{T} u_{0}+\gamma^{T} z_{0}+\delta^{T} d \\
0 & =A x_{0}+B u_{0}+C z_{0}+D d
\end{aligned}
$$




$$
\begin{aligned}
0 & =F x_{0}+G u_{0}+H z_{0}+K d \\
a & \geq M x_{0}+N u_{0}+O z_{0}+P d+I_{\sigma} \tilde{\sigma} \\
d^{l} & \leq d \leq d^{h} \\
u^{l}+U & \leq u_{0} \leq u^{h}-U
\end{aligned}
$$

This system may be solved for the optimum $u_{o}$ and $d$ to determine the optimum dynamic economics $J$.

In summary the linear dynamic economics of process with a fixed controller structure are obtained by:

1. Calculating $\tilde{\sigma}$ and $U$ in response to a disturbance $p$ using frequency response analysis

2. Calculating the dynamic giveaway from the nonlinear steady state optimum economics by solving the above LP for $J$

\subsubsection{Calculation of $\tilde{\sigma}$ and $U$}

To calculate $\tilde{\sigma}$ and $U$, return to the full linear dynamic model. Dropping the objective function and manipulated and design variable bounds, and introducing the slack deviation vector $\sigma$, the system becomes:

$$
\begin{aligned}
\dot{x} & =A x+B u+C z+D d+E p \\
0 & =F x+G u+H z+K d+L p \\
0 & =M x+N u+O z+P d+R p+I_{\sigma} \sigma
\end{aligned}
$$

The slack deviation vector is defined as $\sigma=\Sigma-a$, where $\Sigma$ is the absolute slack vector given by:

$$
a=M x+N u+O z+P d+R p+I_{\sigma} \Sigma
$$

The remainder of the section discusses the calculation of $\tilde{\sigma}$, but all arguments developed apply to $U$.

Prior to examining the system dynamics, the following should be noted:

1. Consider the behaviour of the above linear time invariant model at two steady state operating points (corresponding to different values of $u_{0}$ and $d$ ). By the principle of superposition of linear systems, if these operating points are 
subjected to identical deviation disturbances, the responses, as deviations from the operating points, will be identical. That is, the only difference between the system responses will be the steady state translations. Therefore dynamic analysis of the system is independent of the values of $u_{0}$ and $d$, provided it only considers the deviation behaviour of the system, and not the steady state translation

2. By similar arguments, the design vector $d$ will not appear in the dynamic system as it only makes a steady state contribution to the system

These results imply that the dynamic system can be solved independently of the steady state linear program (although the LP is dependent on the results of the dynamic calculation).

Now take the Laplace transform of the system to examine the dynamic behaviour.

$$
\begin{aligned}
s I_{x} \bar{x} & =A \bar{x}+B \bar{u}+C \bar{z}+E \bar{p} \\
0 & =F \bar{x}+G \bar{u}+H \bar{z}+L \bar{p} \\
0 & =M \bar{x}+N \bar{u}+O \bar{z}+R \bar{p}+I_{\sigma} \bar{\sigma}
\end{aligned}
$$

This system cannot be solved until all of the degrees of freedom have been specified. The number of degrees of freedom of the system equals the number of disturbance variables $n_{p}$ plus the number of manipulated variables $n_{u} . n_{p}$ degrees of freedom are used to specify the dynamic disturbances to the process. The remaining $n_{u}$ degrees of freedom are used as perfect control specifications, falling into two categories:

1. Unused manipulated variables $\left(\bar{u}_{j}=0\right)$

2. Perfect control objectives $\left(\bar{z}_{i}=0\right)$

Once the degrees of freedom of the system have been specified, the maximum amplitudes of the slack variable oscillations in response to a sinusoidal disturbance can be calculated using the frequency response concepts developed previously (section 4.1):

$$
\sigma_{\max }(\omega)=\max _{\Phi}|\sigma(\omega)|=|\Delta(j \omega)| p(\omega)
$$

where $\sigma_{\max }(\omega)$ is the maximum possible amplitude of slack variable oscillation in response to a disturbance of maximum amplitude $p(\omega)$ at frequency $\omega, \Delta$ is the 
matrix relationship between $\bar{\sigma}$ and $\bar{p}$, and $\Phi$ is a set of vectors of disturbance phase lags. Each vector in $\Phi$ corresponds to the phase lags which maximise one element of $\sigma_{\max }$. Note that for this calculation the notation $|\Lambda|$, for some matrix or vector $\Lambda$, is not a norm, but rather the matrix or vector of the magnitudes of the complex elements of $\Lambda$.

$\sigma_{\max }$ is evaluated at one frequency using the above analysis, but for design purposes it is desirable to evaluate $\sigma_{\max }$ over the entire disturbance frequency range $\omega_{d}$. This is possible if the maximum amplitudes of $p$ are known over the disturbance frequency range $\omega_{d}$, i.e. given the maximum amplitude of $p$ at each frequency in $\omega_{d}$,

$$
p_{\max }(\omega)=\max _{p(\omega) \in P(\omega)} p(\omega) \forall \omega \in \omega_{d}
$$

where $P(\omega)$ is the set of all possible disturbances at frequency $\omega$, then the maximum slack amplitudes $\tilde{\sigma}$ over the frequency range can be calculated from:

$$
\begin{aligned}
\tilde{\sigma}_{i} & =\max _{\omega \in \omega_{d}} \sigma_{\max , i}(\omega) \\
& =\max _{\omega \in \omega_{d}}\left(\left[|\Delta(j \omega)| p_{\max }(\omega)\right]_{i}\right)
\end{aligned}
$$

where $i$ refers to the $i^{t h}$ element in the vector, $i=1,2, \ldots, n_{\sigma}$. In brief the method is: evaluate $\sigma_{\max }$ over $\omega_{d}$; set $\tilde{\sigma}_{i}$ to be the maximum value that $\sigma_{\max , i}$ takes over $\omega_{d}$. For the rest of this analysis it will be assumed that $\omega_{d}$ is a single frequency, but as can be seen multiple frequencies or a continuous range of frequencies can be readily handled.

\section{Calculation of $\sigma_{\max }$}

The next step is to consider the calculation of $\sigma_{\max }$ for the case of perfect control or perfect disturbance rejection. This is an ideal case that can be used to estimate the best attainable dynamic economics. For this case we consider a control structure consisting of a subset of the potential measured variables to be maintained at their set point in the face of a given disturbance $p(w)$ (zero deviation) by appropriate manipulation of a subset of the potential manipulated variables. From the previous chapter, a direct relationship between $\bar{\sigma}$ and $\bar{p}$ may be obtained by introducing permutation matrices $Q_{u}$ and $Q_{z}$ which select the perfectly controlled measured variables from $z$ and the inactive manipulated variables from $u$. Using 
these matrices, the following system results without requiring specification of a controller:

$$
\left[\begin{array}{c}
\bar{x} \\
\bar{u} \\
\bar{z} \\
\bar{\sigma}
\end{array}\right]=\left[\begin{array}{cccc}
\left(s I_{x}-A\right) & -B & -C & 0 \\
-F & -G & -H & 0 \\
-M & -N & -O & -I_{\sigma} \\
0 & Q_{u} & 0 & 0 \\
0 & 0 & Q_{z} & 0
\end{array}\right]^{-1}\left[\begin{array}{c}
E \\
L \\
R \\
0 \\
0
\end{array}\right] \bar{p}
$$

This leads to the following expression for $\sigma_{\max }$ (and $u_{\max }$ ) for the perfect control case:

$$
\left[\begin{array}{c}
\max |\bar{x}| \\
u_{\max } \\
\max |\bar{z}| \\
\sigma_{\max }
\end{array}\right]=\left|\left[\begin{array}{cccc}
\left(j \omega I_{x}-A\right) & -B & -C & 0 \\
-F & -G & -H & 0 \\
-M & -N & -O & -I_{\sigma} \\
0 & Q_{u} & 0 & 0 \\
0 & 0 & Q_{z} & 0
\end{array}\right]^{-1}\left[\begin{array}{c}
E \\
L \\
R \\
0 \\
0
\end{array}\right]\right| p(\omega)
$$

A similar result can be derived for a specified controller of the form $u^{\prime}=G_{c}(s) z^{\prime}$, where $u^{\prime}$ and $z^{\prime}$ are the subsets of $u$ and $z$ used in the controller.

In summary, the linear dynamic economic analysis has been formalised as:

1. Linearise a nonlinear dynamic process model at its steady state optimum

2. For a specific control structure and set of disturbances, calculate $\tilde{\sigma}$ and $U$

3. Substitute these values into the steady state linear program derived from the nonlinear problem and solve for the dynamic economics as a deviation (giveaway) from the nonlinear optimum objective function

This procedure provides the basis for the systematic control structure selection method discussed in the next section.

\subsection{MILP control structure selection}

The perfect control ideas in the previous section introduced a method for selection of control structures by varying the permutation matrices $Q_{z}$ and $Q_{u}$. The permutation matrices can be used to select each of the alternative combinations of measured and manipulated variables. Using the $Q$ in this fashion to calculate $\tilde{\sigma}$, it 
is possible to assess the dynamic economics for all of the possible control structures, and choose the optimum control structure directly.

This method is acceptable for problems with a small number of manipulated and measured variables, but rapidly becomes unwieldy for problems with a significant number of potential control structures. Therefore it would be desirable to develop a systematic algorithm for choosing the optimum structure from a large set of manipulated and measured variables. The algorithm should also include screening of the search space to avoid evaluating all of the possible control structures. An algorithm using a hybrid mixed integer linear programming (MILP) method and the perfect control dynamic economics from the previous section is presented as a solution to this problem.

We start with the linear process model developed in the previous section:

$$
\begin{aligned}
\min _{u_{0}, d} J= & \alpha^{T} x_{0}+\beta^{T} u_{0}+\gamma^{T} z_{0}+\delta^{T} d \\
\text { s.t. } \quad \dot{x}= & A x+B u+C z+D d+E p \\
0= & F x+G u+H z+K d+L p \\
a \geq & M x+N u+O z+P d+R p \\
& u^{l} \leq u \leq u^{h} \\
& d^{l} \leq d \leq d^{h}
\end{aligned}
$$

Using the work from the previous section and initially considering a nominal disturbance of amplitude $p(\omega)$ at a nominal disturbance frequency $\omega$, the system equations can be written as:

$$
\begin{aligned}
\min _{u_{0}, d} J & =\alpha^{T} x_{0}+\beta^{T} u_{0}+\gamma^{T} z_{0}+\delta^{T} d \\
0 & =A x_{0}+B u_{0}+C z_{0}+D d \\
0 & =F x_{0}+G u_{0}+H z_{0}+K d \\
a & \geq M x_{0}+N u_{0}+O z_{0}+P d+I_{\sigma} \tilde{\rho} \\
u^{l} & \leq u-\tilde{u} \\
u^{h} & \geq u+\tilde{u} \\
d^{l} & \leq d \leq d^{h} \\
s I_{x} \bar{x} & =A \bar{x}+B \bar{u}+C \bar{z}+E \bar{p} \\
0 & =F \bar{x}+G \bar{u}+H \bar{z}+L \bar{p}
\end{aligned}
$$




$$
0=M \bar{x}+N \bar{u}+O \bar{z}+R \bar{p}+I_{\sigma} \bar{\sigma}
$$

where $\tilde{\rho}$ and $\tilde{u}$ are approximations of the maximum constraint back off.

Setting $s=j \omega$ and considering $\bar{x}, \bar{z}$ and $\bar{\sigma}$ in their complex forms $\bar{x}_{r}+j \bar{x}_{c}$, etc., the frequency response equations may be rewritten as:

$$
\left[\begin{array}{c}
-E \\
-L \\
-R
\end{array}\right]\left[\bar{p}_{r}+j \bar{p}_{c}\right]=\left[\begin{array}{cccc}
\left(A-j \omega I_{x}\right) & B & C & 0 \\
F & G & H & 0 \\
M & N & O & I_{\sigma}
\end{array}\right]\left[\begin{array}{c}
\bar{x}_{r}+j \bar{x}_{c} \\
\bar{u}_{r}+j \bar{u}_{c} \\
\bar{z}_{r}+j \bar{z}_{c} \\
\bar{\sigma}_{r}+j \bar{\sigma}_{c}
\end{array}\right]
$$

or decomposing into real and imaginary parts and dropping $j$ :

$$
\begin{aligned}
-E \bar{p}_{r} & =A \bar{x}_{r}+B \bar{u}_{r}+C \bar{z}_{r}+\omega I_{x} \bar{x}_{c} \\
-L \bar{p}_{r} & =F \bar{x}_{r}+G \bar{u}_{r}+H \bar{z}_{r} \\
-R \bar{p}_{r} & =M \bar{x}_{r}+N \bar{u}_{r}+O \bar{z}_{r}+I_{\sigma} \bar{\sigma}_{r} \\
-E \bar{p}_{c} & =-\omega I_{x} \bar{x}_{r}+A \bar{x}_{c}+B \bar{u}_{c}+C \bar{z}_{c} \\
-L \bar{p}_{c} & =F \bar{x}_{c}+G \bar{u}_{c}+H \bar{z}_{c} \\
-R \bar{p}_{c} & =M \bar{x}_{c}+N \bar{u}_{c}+O \bar{z}_{c}+I_{\sigma} \bar{\sigma}_{c}
\end{aligned}
$$

Before the problem formulation is complete the following relationships must be specified:

- The perfect control requirements

- The functional relationships $\tilde{\rho}\left(\bar{\sigma}_{r}, \bar{\sigma}_{c}\right)$ and $\tilde{u}\left(\bar{u}_{r}, \bar{u}_{c}\right)$

\subsubsection{Perfect control specifications}

There are two possible types of perfect control specifications:

1. A manipulated variable is inactive (unused for control).

2. A measured variable is controlled perfectly.

In both cases the appropriate element of $u$ or $z$ is constrained to zero. If a perfect control specification is not made on a variable, then that variable is unconstrained (corresponding to uncontrolled measured variables or active manipulated variables). Introduce the integer vectors $X$ and $\mathcal{X}$ to describe the perfect control specifications, where: 
$X_{j}=0$ if control variable $j$ is inactive

$\mathcal{X}_{i}=1$ if measurement $i$ is perfectly controlled

Using these definitions of $X$ and $\mathcal{X}$, and noting that the perfect control requirements may be written as: $\left|\bar{z}_{i}\right|=0$ or $\bar{z}_{r, i}=\bar{z}_{c, i}=0$ for measured variables and $\left|\bar{u}_{j}\right|=0$ or $\bar{u}_{r, j}=\bar{u}_{c, j}=0$, then the perfect control constraints may be formulated as:

$$
\left.\begin{array}{r}
-\left(1-\mathcal{X}_{i}\right) \bar{z}_{i}^{h} \leq \bar{z}_{r, i} \leq\left(1-\mathcal{X}_{i}\right) \bar{z}_{i}^{h} \\
-\left(1-\mathcal{X}_{i}\right) \bar{z}_{i}^{h} \leq \bar{z}_{c, i} \leq\left(1-\mathcal{X}_{i}\right) \bar{z}_{i}^{h}
\end{array}\right\} \quad \forall i=1,2, \ldots, n_{z}
$$

where $\bar{z}^{h}$ is a sensible upper bound on the expected magnitude of $|\bar{z}| . \quad \bar{u}^{h}$ is a similar bound on $|\bar{u}|$ and can also be used as an upper bound on the manipulated variable rangeability. To satisfy the degrees of freedom requirement, $n_{u}$ perfect control specifications are required, resulting in the constraint:

$$
\sum_{i=1}^{n_{z}} \mathcal{X}_{i}-\sum_{j=1}^{n_{u}} X_{j}=0
$$

Finally, the integer vectors can also be used to account for the costs of installing actuators and sensors for control purposes by modifying the objective function to:

$$
\min _{u_{0}, d, X, \mathcal{X}} J=\alpha^{T} x_{0}+\beta^{T} z_{0}+\gamma^{T} d+\epsilon^{T} X+\varepsilon^{T} \mathcal{X}
$$

where $\epsilon$ and $\varepsilon$ are respectively the actuator and sensor costs.

So far this problem is a mixed integer linear programming (MILP) formulation, as all of the constraints are linear. Problems of this type may be solved using the branch and bound algorithm (Beale, 1977). However, we require the functional relationships for $\tilde{\rho}(\omega)$ and $\tilde{u}(\omega)$ before the problem is fully specified. The following discussion will only consider $\tilde{\rho}(\omega)$, as the arguments presented also extend to $\tilde{u}(\omega)$.

\subsubsection{Linear lower bound on $\tilde{\rho}$}

Ideally $\tilde{\rho}(\omega)$ is given by the maximum expected amplitude of oscillation of the slack variables. From the previous section, this is given by:

$$
\max _{\Phi}|\bar{\sigma}(\omega)|=|\Delta(j \omega)| p(\omega)
$$


which requires the evaluation of complex magnitudes, a nonlinear calculation. Use of this constraint will destroy the linear properties of the problem formulation, and will result in a complex mixed integer nonlinear program (MINLP) with discontinuous constraints and first derivatives, a particularly difficult problem to solve. Therefore it is desirable to use some approximation which maintains the linear properties, but still allows location of the optimal solution.

Two properties may be used to circumvent this problem:

1. Consider the linear programs LP1 and LP2:

$$
\begin{aligned}
& \min _{q} J(L P 1)=a^{T} q \\
& b \geq \Lambda q+I_{\sigma} \tilde{\rho}(q)
\end{aligned}
$$

and

$$
\begin{aligned}
\min _{q} J(L P 2) & =a^{T} q \\
b & \geq \Lambda q+I_{\sigma} \eta
\end{aligned}
$$

Where $\tilde{\rho}$ is defined as above. If the vector of linear variables $\eta$ can be constrained such that:

$$
\begin{aligned}
& \eta \geq \Gamma q \\
& \eta<\infty
\end{aligned}
$$

and it can be shown that the lower bound on $\eta$ for any given vector $\hat{q}\left(\eta^{l}=\Gamma \hat{q}\right)$ is also a lower bound on $\tilde{\rho}\left(\tilde{\rho} \geq \eta^{l}\right)$, then the following inequality holds for the optimum objective functions of the linear programs:

$$
J^{*}(L P 1) \geq J^{*}(L P 2)
$$

as $\eta_{i}$ will always be less than $\tilde{\rho}_{i}$ for an active constraint $i$ at the optimum $q^{*}$.

2. Whenever an integer solution is generated by the MILP solution procedure, a control structure is fully specified. That is, the permutation matrices $Q_{z}$ and $Q_{u}$ can be generated from $X$ and $\mathcal{X}$, and therefore the perfectly controlled objective function corresponding to the integer solution (control structure) can be calculated using the methods from the previous section. 
Using these two properties, an algorithm for the rigorous solution of the control structure selection problem can be proposed. Assuming a linear lower bound on the maximum slack variation can be calculated, set $\tilde{\rho}$ to this lower bound in the MILP formulation. That is, use $\tilde{\rho}$ as an approximation of the maximum slack variation $\tilde{\sigma}$. This MILP formulation calculates optimistic objective functions (by the first property). When the MILP solution method produces an integer solution, use the perfect control analysis from the previous section to calculate the actual objective function for the given control structure (by the second property) and use the actual objective function as the entry cost for integer solutions in the MILP solution method, rather than the optimistic objective function generated previously. This is an incomplete description of the algorithm, as a few more steps are required to ensure the optimal control structure is chosen, but it is sufficient as an initial outline for the justification of the use of the MILP approximation.

To maintain the desirable features of an MILP formulation and still obtain a rigorous solution, a linear lower bound for $\tilde{\rho}$ must be developed. A possible lower bound is given by the following steps:

1. Consider a single element of the vector $\bar{\sigma}$, obtained by solution of equations $5.2-$ 5.7 for an arbitrary disturbance $\bar{p}=\bar{p}_{r}+j \bar{p}_{c}$ with amplitude $p(\omega)$ (assuming perfect control specifications have been made on $z$ and $u$ to use the remaining $n_{u}$ degrees of freedom). For this arbitrary disturbance it can be stated:

$$
\max _{\phi}|\bar{\sigma}(\omega)| \geq|\bar{\sigma}(\omega)|
$$

2. A lower bound on $|\bar{\sigma}(\omega)|$ is obtained from the complex form of $\bar{\sigma}(\omega)=a+j b$ :

$$
\begin{aligned}
& |\bar{\sigma}(\omega)| \geq|a| \\
& |\bar{\sigma}(\omega)| \geq|b|
\end{aligned}
$$

3. A linear lower bound on the absolute value of $a$ is given by:

$$
\begin{aligned}
& |a| \geq a \\
& |a| \geq-a
\end{aligned}
$$

and similar bounds may be written for $|b|$. 
For convenience of calculation, the arbitrary disturbance is taken as $\bar{p}_{r}=p(\omega)$ and $\bar{p}_{c}=0$.

Using the above sequence of lower bounds, we obtain the following linear lower bound on the maximum slack variation $\tilde{\rho}$ :

$$
\begin{aligned}
& \tilde{\rho} \geq \bar{\sigma}_{\boldsymbol{r}} \\
& \tilde{\boldsymbol{\rho}} \geq \bar{\sigma}_{c} \\
& \tilde{\boldsymbol{\rho}} \geq-\bar{\sigma}_{r} \\
& \tilde{\boldsymbol{\rho}} \geq-\bar{\sigma}_{c}
\end{aligned}
$$

where $\left\{\bar{\sigma}_{r}, \bar{\sigma}_{c}\right\}$ are obtained from the solution of equations 5.2-5.7. A similar result holds for $\tilde{u}$. Given this result, an MILP approximation of the control structure selection problem may be written. However, as was noted in chapter 3 , it would be desirable to include some screening technique to eliminate infeasible structures without examination. This may be achieved by the introduction of structural connectivity constraints.

\subsubsection{Connectivity constraints}

The current formulation allows selection of any $n_{u}$ perfectly controlled variables as a valid integer solution. This is a combinatorial problem of selecting $n_{u}$ variables out of $n_{u}+n_{z}$, where:

$$
\left(\begin{array}{c}
n_{u}+n_{z} \\
n_{u}
\end{array}\right)=\frac{\left(n_{u}+n_{z}\right) !}{\left(n_{u}+n_{z}-n_{u}\right) ! n_{u} !}
$$

For example for a problem of selecting 20 degrees of freedom from 40 manipulated and measured variables, the number of integer feasible solutions is $1.378 \times 10^{11}$, which is a large problem. It would be desirable to reduce the feasible integer search space using structural information about the relationships between the inputs and outputs (input/output connectivity). The use of structural information would be maximised by ensuring that the transfer function between the active manipulated variables and the perfectly controlled measurements is structurally non-singular. During the development phase of this method, it was not clear how to pose the requirement of structural non-singularity in the current problem representation. However, it 
proved possible to generate a set of weaker structural connectivity constraints for use in this problem representation.

In words, these constraints are: for every measured variable selected to be perfectly controlled, at least one manipulated variable which affects the measured variable must be active. Similarly for each active manipulated variable selected, at least one measured variable that it affects must be perfectly controlled. Given the structural transfer function, $G_{\text {structural }}$, generated from the transfer function at frequency $\omega$ :

$$
G_{\text {structural }}=\left[\begin{array}{cccc}
a_{11} & a_{12} & \ldots & a_{1 m} \\
a_{21} & a_{22} & & a_{2 m} \\
\vdots & \vdots & \ddots & \vdots \\
a_{n 1} & a_{n 2} & \ldots & a_{n m}
\end{array}\right]
$$

where $a_{i j}=1$ if there is a nonzero gain between manipulated variable $j$ and measured variable $i$, and $a_{i j}=0$ if the gain is zero, it is possible to generate the connectivity constraints.

First consider the constraint that for each perfectly controlled measured variable there must be at least one active manipulated variable that affects the measured variable. Manipulated variable $j$ is active when $X_{j}=1$. Therefore when a manipulated variable which affects a measured variable is active, the following inequality holds:

$$
\sum_{j=1}^{n_{u}} a_{i j} X_{j} \geq 1
$$

This inequality must hold for manipulated variable $i$ to be perfectly controlled. Therefore it can be stated:

If $\mathcal{X}_{i}=1$ (perfectly controlled) then

$$
\sum_{j=1}^{n_{u}} a_{i j} X_{j} \geq 1
$$

If $\mathcal{X}_{i}=0$ then

$$
\sum_{j=1}^{n_{u}} a_{i j} X_{j} \geq 0
$$

Combining into a single constraint:

$$
\sum_{j=1}^{n_{u}} a_{i j} X_{j}-\mathcal{X}_{i} \geq 0 \quad \forall i=1,2, \ldots, n_{z}
$$


Now consider the constraint that each active manipulated variable must affect at least one of the perfectly controlled measured variables. The two conditions for this constraint are:

If $X_{j}=1$ (manipulated variable active) then

$$
\sum_{i=1}^{n_{2}} a_{i j} \mathcal{X}_{i} \geq 1
$$

If $X_{j}=0$ then

$$
\sum_{i=1}^{n_{2}} a_{i j} \mathcal{X}_{i} \geq 0
$$

Combining the conditions:

$$
\sum_{i=1}^{n_{2}} a_{i j} \mathcal{X}_{i}-X_{j} \geq 0 \forall j=1,2, \ldots, n_{u}
$$

With these connectivity constraints, the complete MILP formulation of the problem can be written. Note that this is a weaker set of constraints than structural nonsingularity, and further investigation should be carried out to try to implement this structural analysis in the MILP.

A brief summary of both the MILP and perfect control subproblems is given before outlining the complete algorithm for solution of the control structure selection problem.

\section{Full MILP formulation}

$$
\begin{aligned}
\left.\begin{array}{rl}
\min _{u_{0}, d, X, \mathcal{X}} J_{M} & =\alpha^{T} x_{0}+\beta^{T} z_{0}+\gamma^{T} d+\epsilon^{T} X+\varepsilon^{T} \mathcal{X} \\
\sum_{i=1}^{n_{z}} \mathcal{X}_{i}-\sum_{j=1}^{n_{u}} X_{j} & =0 \\
\bar{z}_{i}^{h} \geq \mathcal{X}_{i} \bar{z}_{i}^{h}+\bar{z}_{r, i} \\
\bar{z}_{i}^{h} \geq \mathcal{X}_{i} \bar{z}_{i}^{h}+\bar{z}_{c, i} \\
\bar{z}_{i}^{h} \geq \mathcal{X}_{i} \bar{z}_{i}^{h}-\bar{z}_{r, i} \\
\bar{z}_{i}^{h} \geq \mathcal{X}_{i} \bar{z}_{i}^{h}-\bar{z}_{c, i}
\end{array}\right\} \quad \forall i=1,2, \ldots, n_{z}
\end{aligned}
$$




$$
\begin{aligned}
& \left.\begin{array}{c}
X_{j} \bar{u}_{j}^{h} \geq \bar{u}_{r, j} \\
X_{j} \bar{u}_{j}^{h} \geq \bar{u}_{c, j} \\
X_{j} \bar{u}_{j}^{h} \geq-\bar{u}_{r, j} \\
X_{j} \bar{u}_{j}^{h} \geq-\bar{u}_{c, j}
\end{array}\right\} \quad \forall j=1,2, \ldots, n_{u} \\
& \sum_{j=1}^{n_{u}} a_{i j} X_{j}-\mathcal{X}_{i} \geq 0 \quad \forall i=1,2, \ldots, n_{z} \\
& \sum_{i=1}^{n_{z}} a_{i j} \mathcal{X}_{i}-X_{j} \geq 0 \quad \forall j=1,2, \ldots, n_{u} \\
& 0=A x_{0}+B u_{0}+C z_{0}+D d \\
& 0=F x_{0}+G u_{0}+H z_{0}+K d \\
& a \geq M x_{0}+N u_{0}+O z_{0}+P d+I_{\sigma} \tilde{\rho} \\
& u^{l} \leq u_{0}-\tilde{u} \\
& u^{h} \geq u_{0}+\tilde{u} \\
& d^{l} \leq d \leq d^{h} \\
& -E p(\omega)=A \bar{x}_{r}+B \bar{u}_{r}+C \bar{z}_{r}+\omega I_{x} \bar{x}_{c} \\
& -L p(\omega)=F \bar{x}_{r}+G \bar{u}_{r}+H \bar{z}_{r} \\
& -R p(\omega)=M \bar{x}_{r}+N \bar{u}_{r}+O \bar{z}_{r}+I_{\sigma} \bar{\sigma}_{r} \\
& 0=-\omega I_{x} \bar{x}_{r}+A \bar{x}_{c}+B \bar{u}_{c}+C \bar{z}_{c} \\
& 0=F \bar{x}_{c}+G \bar{u}_{c}+H \bar{z}_{c} \\
& 0=M \bar{x}_{c}+N \bar{u}_{c}+O \bar{z}_{c}+I_{\sigma} \bar{\sigma}_{c} \\
& \tilde{\rho} \geq \bar{\sigma}_{\boldsymbol{r}} \\
& \tilde{\rho} \geq-\bar{\sigma}_{r} \\
& \tilde{\rho} \geq \bar{\sigma}_{c} \\
& \tilde{\rho} \geq-\bar{\sigma}_{c} \\
& \tilde{u} \geq \bar{u}_{r} \\
& \tilde{u} \geq-\bar{u}_{r} \\
& \tilde{u} \geq \bar{u}_{c} \\
& \tilde{u} \geq-\bar{u}_{c}
\end{aligned}
$$




\section{MILP solution techniques}

General techniques for the solution of MILP problems are discussed in Nemhauser and Wolsey (1988). The techniques of explicit enumeration, branch and bound (Beale, 1977) and Benders decomposition (Benders, 1962) will be reviewed briefly.

\section{Explicit enumeration}

Explicit enumeration consists of generating all possible combinations of the integer variables, and then solving the remaining linear program with respect to the continuous variables for each combination. The optimum solution corresponds to the integer combination with the best continuous objective function. This approach is not suitable for the problem posed here, as the intention of using an integer programming formulation was to avoid examination of all possible control structures.

\section{Benders decomposition}

Benders decomposition (Benders, 1962) solves the MILP in two phases: an integer master problem and a continuous subproblem. The integer master problem contains all of the pure integer constraints, and the subproblem contains the remaining constraints and the objective function. Assuming a minimisation problem, a simple outline of the algorithm is given in the following steps:

1. Choose an initial integer solution. Set the upper bound on the objective function $U B D$ to positive infinity (a large number).

2. Solve the continuous subproblem for the fixed set of integer variables. If the optimal objective function is lower than the upper bound, $U B D$, then set $U B D$ to the optimal objective function and record the integer vector as the best integer vector found so far

3. Augment the master problem using Lagrangian information from the continuous problem, and add an integer cut to ensure the most recent integer solution is not re-examined 
4. Solve the master integer program using branch and bound or another integer programming method to yield a new lower bound on the objective function, $L B D$, and a new integer solution

5. Terminate if the lower bound $L B D$ is larger than the upper bound $U B D$, otherwise return to step 2

The advantage of this algorithm is that it replaces a complex MILP with smaller, more easily solved integer programs and associated subproblems. An integer cut for the binary integer vector $Z$ is given by (Grossmann, 1990):

$$
\sum_{i \in B} Z_{i}-\sum_{j \in N} Z_{j} \leq|B|-1
$$

where the indices in the set $B$ correspond to the elements of $Z$ with unity values, and the indices in $N$ correspond to the elements in $Z$ with zero values. $|B|$ is the number of elements in $B$.

Benders decomposition was tested as a possible solution method for the control structure selection MILP, but it was found to have significantly poorer convergence properties than branch and bound, and therefore it was eliminated as a potential solution technique.

\section{Branch and bound}

Branch and bound techniques solve MILP problems by starting with a relaxed linear program. That is, the integrality requirements on the integer variables are released, and they are treated as continuous variables with simple upper and lower bounds. The integrality condition is then gradually enforced on the integer variables in a search procedure until integer solutions are generated (branching). Intermediate information is used to eliminate poor solutions without full evaluation (bounding). The algorithms for both depth first and breadth first branch and bound will be summarised briefly. Considering depth first search with a binary integer vector $Z$ :

1. Set the entry cost to positive infinity (again considering a minimisation problem)

2. Solve the fully relaxed LP (i.e. $0 \leq Z \leq 1$ ) 
3. If the elements of $Z$ are all integer, then the solution to the fully relaxed LP is the optimal integer solution, and the procedure is terminated. Similarly terminate if the problem is infeasible. Otherwise make the relaxed problem the current branch

4. If the objective function of the current problem is less than the entry cost then branch on a non-integer element of $Z$. That is, an element $i$ of $Z$ where $0<Z_{i}<1$. A branch forces the integrality of $Z_{i}$ by solving two augmented LP problems with $Z_{i}=0$ and $Z_{i}=1$ respectively. These constraints continue to be associated with the branches as the search continues. Rules for choosing the element, $i$, to branch on are discussed in general integer programming texts (Williams, 1990; Nemhauser and Wolsey, 1988).

- Eliminate branches that either are infeasible or have objective functions greater than the entry cost from further consideration (pruning the search tree)

- If either branch has an integer solution, then make the integer solution with the best (lowest) objective function the best solution found in the search to date, and replace the entry cost with the objective function of this integer solution. Remove the integer branches from further consideration (fathoming the branch)

- If both branches are still under consideration, then add both branches to the top of the "stack" of branches for future examination. Rules for the order of branch placement on the stack are typically based on objective functions and approach of the relaxed solution to integrality (Nemhauser and Wolsey, 1988)

- If one branch remains under consideration, then place it on top of the stack

5. If the stack is empty, then the best solution found so far is the optimum, and the procedure terminates. Otherwise the top element on the stack is made the current branch, and step 4 is repeated

For breadth first branch and bound, the push/pop stack is replaced by an ordered stack. That is, an index of the quality of each branch is generated, and the 
stack is sorted from the top in order of decreasing quality of relaxed solutions. The index may be the objective function, or a combination of the objective function and a measure of integer feasibility of the branch.

Depth first search will rapidly fathom a tree, and is best when the solution of the problem is only required to within a margin of optimality (e.g. any solution with an objective function within $95 \%$ of the true optimum is adequate), or for problems with degenerate optima - both conditions typical of batch scheduling (Shah, 1992). Breadth first is better when an exact optimum is required for a problem with a unique solution, as is typically the case for the control structure selection problem. For this type of problem, it guarantees the minimum number of LP solutions to locate the optimum, as the search does not have the potential to traverse parts of the search tree not containing the global optimum. Breadth first branch and bound was found to have adequate performance when applied to the control structure selection MILP, and has been employed.

\subsubsection{Perfect control problem}

The following problem is solved at an integer solution $X, \mathcal{X}$.

$$
\left[\begin{array}{c}
\max |x| \\
U \\
\max |z| \\
\tilde{\sigma}
\end{array}\right]=\left|\left[\begin{array}{cccc}
j \omega I-A & -B & -C & 0 \\
-F & -G & -H & 0 \\
-M & -N & -O & -I_{\sigma} \\
0 & Q_{u} & 0 & 0 \\
0 & 0 & Q_{z} & 0
\end{array}\right]^{-1}\left[\begin{array}{c}
E \\
L \\
R \\
0 \\
0
\end{array}\right]\right| p(\omega)
$$

The unity elements in $Q_{u}$ and $Q_{z}$ correspond to the unity elements in the $X$ and $\mathcal{X}$ vectors. The location of the unity element in each row corresponds to the appropriate elements of $u$ and $z$. This system is solved for $\tilde{\sigma}$ and $U$ which are substituted into the following LP to obtain the optimum economics of the linear solution:

$$
\begin{aligned}
\min _{u_{0}, d} J_{p c} & =\alpha^{T} x_{0}+\beta^{T} z_{0}+\gamma^{T} d+\delta^{T} p_{0}+\epsilon^{T} X+\varepsilon^{T} \mathcal{X} \\
0 & =A x_{0}+B u_{0}+C z_{0}+D d \\
0 & =F x_{0}+G u_{0}+H z_{0}+K d \\
a & \geq M x_{0}+N u_{0}+O z_{0}+P d+\tilde{\sigma} \\
u^{l}+U & \leq u \leq u^{h}-U
\end{aligned}
$$




$$
d^{l} \leq d \leq d^{h}
$$

To date the problem formulation has only considered a nominal disturbance amplitude $p(\omega)$ at nominal frequency $\omega$. However it would be desirable to obtain the best controller structure with respect to disturbance amplitudes $p_{\max }$ over the disturbance frequency set $\omega_{d}$ (equation 5.1 ). This can be achieved by calculating $\sigma_{\max }$ and $u_{\max }$ at each frequency point in $\omega_{d}$, and the maximum values over the frequency range are be obtained using: $\tilde{\sigma}=\max _{\omega \in \omega_{d}} \sigma_{\max }(\omega)$, etc.

The solution of this problem requires a matrix inversion and the solution of an LP.

\subsubsection{MILP control structure selection algorithm}

Two problems have been posed in this section: A perfect control problem (section 5.2.4) whose solution yields an estimate of the attainable dynamic economics for a given control structure, and a MILP problem (section 5.2.3) that generates optimistic (lower) bounds on the perfect control dynamic economics of partially and fully specified control structures. At this point it is desirable to amalgamate these to solve the problem of selecting the optimal perfect control structure. This problem may be solved by using a hybrid MILP technique as follows.

Solve the MILP problem (section 5.2.3) using breadth first branch and bound until an integer solution is found with $J_{M}$ less than the entry cost (including the fully relaxed solution). However, do not continue the branch and bound at this point. Instead solve the perfect control problem (section 5.2.4) to generate $J_{p c}$ as the objective function corresponding to the integer solution, and use this to compare with the entry cost. Whether or not the current solution replaces the best solution, add an integer cut for the current solution to ensure that the solution is not re-examined, and then repeat the branch which generated the integer solution. If no further integer solutions are generated with $J_{M}$ less than the entry cost, then proceed with the branch and bound. That is, use $J_{M}$ to determine if branches should be pruned as normal, and only examine the perfect control problem if there is an integer solution with $J_{M}$ better than the entry cost.

The hybrid approach is used as the MILP only generates an optimistic estimate of the perfectly controlled objective function. This leads to a requirement 
to re-examine branches once an integer solution has been located (true branch and bound never re-examines branches). This is because from some arbitrary branch level, the MILP may find an optimal integer solution $J_{M}^{1}$ where $J_{M}^{1}<J_{0}$ (the current entry cost), with a corresponding perfect control objective function $J_{p c}^{1}$. From the same branch there may also be another feasible integer solution $J_{M}^{2}$ where it also holds that $J_{M}^{2}<J_{0}\left(J_{M}^{2} \geq J_{M}^{1}\right.$ by definition of the optimum), with perfect control objective function $J_{p c}^{2}$. In this case it is possible that $J_{p c}^{2} \leq J_{p c}^{1}$ and either or both may be less than $J_{0}$. That is the optimal linear solution may not correspond to the optimal perfect control solution for any particular branch. This leads to the slight modification of the branch and bound algorithm to ensure that the optimal perfect control solution is found.

As a final point, it should be emphasised that the MILP analysis should only be used as a screening tool for prediction of economically sound control structures. The reasons for this are as follows:

- The analysis does not examine the controllability of the process.

- The analysis only calculates an estimate of the dynamic economics.

- The linearisation only has a limited accuracy for perturbations from the linearisation point.

Because of these limitations, it is proposed that the analysis should be used as follows: The design engineer chooses the number of control structures which are to be examined in detail ( $n_{\text {designs }}$ ) as an input to the analysis program; the program then generates the $n_{\text {designs }}$ control structures with the best dynamic economics. These structures are then all subjected to controllability analyses, or are used as control structures for nonlinear dynamic economic analysis. The results of these analyses should then be used to select the best control structure.

\subsubsection{Implementation}

The above algorithm has been implemented using MINOS (Murtagh and Saunders, 1983) as the LP solver and using modified branch and bound routines developed by Shah (1992) for the solution of the MILP problems. All equality relations in the MILP (with the exception of the degrees of freedom constraint) 
were eliminated to improve speed of solution, and have only been included in this chapter for convenience of representation and completeness. The branch stack was ordered by objective function. A number of rules for the selection of the branching variable were tested, and a routine based on the solution analysis of Shah (1992) was found to perform adequately.

In an attempt to improve MILP solution performance, a tighter MILP formulation was developed, including linear estimates of $\tilde{\sigma}$ with a maximum relative error of $2 \%$. The formulation reduced the number of LP solutions required to locate the optimal solution by a factor of 6 for a small test case, but increased the time required to locate the optimal structure by a factor of 4 compared to the previously posed approach applied to the same problem. As a result no further work on the formulation was carried out. This formulation is summarised in appendix C.

\subsection{Summary}

This chapter formalised the dynamic economic analysis developed in chapter 4 , and removed the deficiencies of infeasible constraint back off and the handling of inactive constraints. This analysis was then proposed as the basis for a hybrid MILP control structure selection problem, including a trade off between control costs and benefits. The performance of the MILP technique will be tested in the following chapter. 


\section{Chapter 6}

\section{MILP case studies}

This chapter presents the results of two case studies used to test the MILP control structure selection algorithm proposed in the previous chapter.

\subsection{Double effect evaporator case study}

This case study considers a concentration operation based on the double effect evaporator problem outlined in the SPEEDUP Casebook (Prosys Technology, 1989). The concentration of a solution of $A$ is to be raised from $3.5 \%$ to $10 \%$. The process objective is to optimise the process throughput versus the operating costs. A process flow diagram is given in figure 6.1. The liquor is fed from an upstream process, into the first evaporator through the feed pump and feed valve. Utility steam is supplied to the first effect, and steam to the second effect is supplied by the first effect vapour. The liquor product of the first effect is fed to the second effect through the intermediate pump and intermediate valve. The product liquor is then fed through a product pump and valve to a downstream storage facility. $L V 1, L V 2, L V 3, V V 1, V V 2, V V 3$ are manual valves, which may be replaced by control valves if required.

A dynamic model of the process was written using SPEEDUP. The process model and physical characteristics of the units are outlined in appendix $\mathrm{D}$. The next step was steady state process optimisation. It was assumed that the evaporator plant was available as a scrapped unit. Therefore, it was decided to optimise the annual operating costs, as there was no capital consideration. The product has an internal transfer price of $£ 250$ /tonne of $A$ concentrated from $3.5 \%$ to $10 \%$. Electricity was rated at $£ 0.02 / \mathrm{kWhr}$, and utility steam at $£ 2 /$ tonne. The pumps used in the process have an efficiency of $80 \%$. The operating inventory was assumed to have a negligible value. The process objective function is given by:

Maximise Value of $A$ produced - Steam costs - Pumping costs 


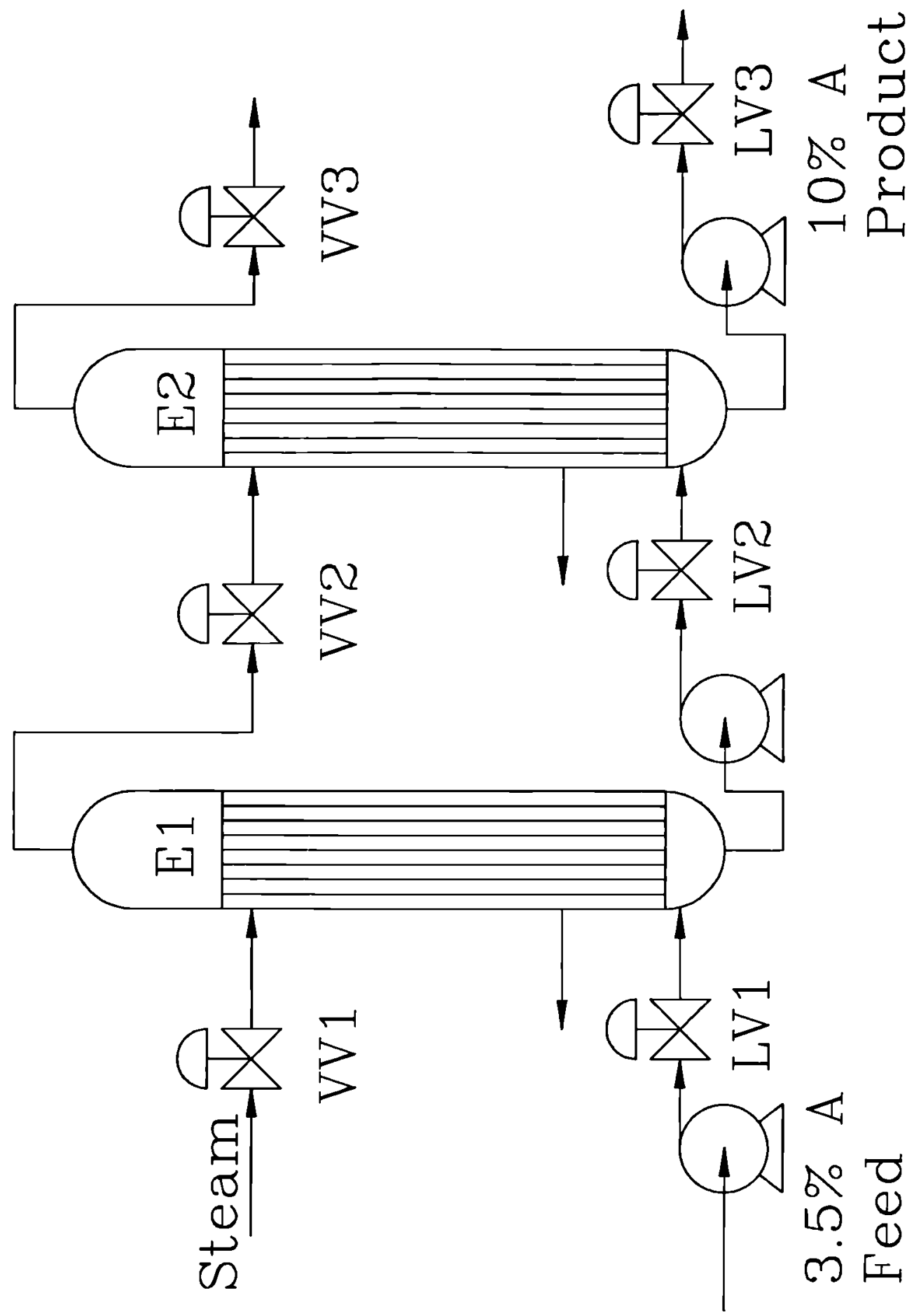

Figure 6.1: Double effect evaporator flowsheet 
For a yearly basis of 8000 operating hours, the objective function is given by:

$$
\max \left[120 \times F_{A}-0.96 \times F_{\text {steam }}-0.003333 \times\left(\frac{\Delta P_{f} F_{f}}{\rho_{f}}+\frac{\Delta P_{i} F_{i}}{\rho_{i}}+\frac{\Delta P_{p} F_{p}}{\rho_{p}}\right)\right]
$$

where

$F_{A}$ is the production of $A$ in $\mathrm{kg} / \mathrm{min}$

$F_{\text {steam }}$ is the feed steam rate to the plant in $\mathrm{kg} / \mathrm{min}$

$\Delta P_{k}$ is the head raised across pump $k$ in $\mathrm{kPa}$

$F_{k}$ is the flow through pump $k$ in $\mathrm{kg} / \mathrm{min}$

$\rho_{k}$ is the density of stream $F_{k}$ in $\mathrm{kg} / \mathrm{m}^{3}$

$f, i, p$ feed, intermediate and product pumps

The power requirement of the pumps is the volumetric flow times the head raised divided by the pump efficiency (Sinnot, 1983). The free variables for the optimisation are the valve coefficients of the liquid and vapour line valves (corresponding to the choice of valve position), as the parameters of the process items are fixed.

A number of constraints were imposed on the operation of the process:

1. The liquor volume in the evaporators must remain within the liquor freeboard (above the steam chest and below the vapour hemisphere of the evaporator $3.25 \leq$ Volume $\leq 4.7 \mathrm{~m}^{3}$ )

2. There must be a five degree Celsius temperature difference between the steam chest and the process liquor for acceptable heat transfer

3. The solute $A$ is subject to thermal decay, and as a result the maximum possible temperature in the evaporators is $95^{\circ} \mathrm{C}$

4. The process must produce at least $3.5 \mathrm{~kg} / \mathrm{min}$ of $A$ in a solution with a minimum concentration of $10 \%$ by weight of $A$

5. During initial optimisation runs, the pressure drop between effects on vapour lines $V V 1$ and $V V 2$ was pushed towards zero, as this maximised vaporisation. However this reduced the sensitivity of the process to changes in the vapour 
valve coefficients (i.e. no control could be exerted), and added the possibility of vapour flow reversal under disturbance conditions. To eliminate these effects, the pressure drop over all vapour line valves was required to be at least $5 \mathrm{kPa}$

6. The process pumps were required to operate within the design limits of maximum flow, maximum pressure and minimum pressure across the pump (appendix D)

7. The squares of the valve coefficients have simple lower and upper bounds of 5.0 and 1000.0

The nominal operating conditions for the process are given in table 6.1.

\begin{tabular}{|l|l|}
\hline Feed pressure & $100 \mathrm{kPa}$ \\
Feed temperature & $88^{\circ} \mathrm{C}$ \\
Feed composition $A$ & 3.5 weight $\%$ \\
Steam temperature & $105^{\circ} \mathrm{C}$ \\
Liquor product pressure & $250 \mathrm{kPa}$ \\
Final effect vapour product pressure & $45 \mathrm{kPa}$ \\
\hline
\end{tabular}

Table 6.1: Nominal evaporator operating conditions

The optimal profit from the process is $£ 457,000$ per annum, with the optimal operating conditions summarised in table 6.2 , and the active constraints and Lagrange multipliers summarised in table 6.3.

\subsubsection{MILP selection of optimal control structure}

Prior to linearisation of the nonlinear model, the potential measured and manipulated variables must be identified. For this case study 23 potential measured variables were identified, and are summarised in table 6.4. Note that it has been assumed that the feed liquor flow and composition cannot be measured directly, and that the liquor composition and/or component flows can only be measured at the intermediate and product valves. The six valve coefficients were identified as potential manipulated variables. This results in ${ }^{29} C_{6}$ or approximately 470,000 potential control structures. After identifying the potential measured and manipulated variables, the nonlinear model was linearised at the steady state optimum using the CDI linearisation package provided with SPEEDUP. The six valve coefficients were 


\begin{tabular}{|c|c|c|c|}
\hline Process feed & $\begin{array}{l}88^{\circ} \mathrm{C} \\
100 \mathrm{kPa} \\
113.5 \mathrm{~kg} / \mathrm{min} \text { water } \\
4.1 \mathrm{~kg} / \mathrm{min} \text { solute }\end{array}$ & E1 & $\begin{array}{l}2.56 \mathrm{~m}^{3} \text { vapour } \\
3.25 \mathrm{~m}^{3} \text { liquor } \\
92^{\circ} \mathrm{C} \\
74.5 \mathrm{kPa}\end{array}$ \\
\hline \multirow[t]{2}{*}{ Steam feed } & \multirow{2}{*}{$\begin{array}{l}105^{\circ} \mathrm{C} \\
120.8 \mathrm{kPa} \\
38.4 \mathrm{~kg} / \mathrm{min}\end{array}$} & $V V 1$ & $\begin{array}{l}P_{o}=115.8 \mathrm{kPa} \\
C_{v}^{2}=294.9\end{array}$ \\
\hline & & Feed pump & $P_{o}=194.6 \mathrm{kPa}$ \\
\hline \multirow[t]{2}{*}{$\begin{array}{l}\text { Intermediate } \\
\text { pump }\end{array}$} & \multirow{2}{*}{$\begin{array}{l}76.1 \mathrm{~kg} / \mathrm{min} \text { water } \\
4.1 \mathrm{~kg} / \mathrm{min} \text { solute } \\
P_{i}=171.2 \mathrm{kPa} \\
P_{\mathrm{o}}=275 \mathrm{kPa}\end{array}$} & $L V 1$ & $\begin{array}{l}P_{o}=163.3 \mathrm{kPa} \\
C_{v}^{2}=441.3\end{array}$ \\
\hline & & \multirow[t]{2}{*}{$V V 2$} & $\begin{array}{l}P_{o}=69.5 \mathrm{kPa} \\
C_{v}^{2}=280.6\end{array}$ \\
\hline \multirow[t]{2}{*}{$L V 2$} & \multirow{2}{*}{$\begin{array}{l}P_{o}=139.2 \mathrm{kPa} \\
C_{v}^{2}=47.4\end{array}$} & & $37.5 \mathrm{~kg} / \mathrm{min}$ \\
\hline & & \multirow[t]{2}{*}{$\overline{\mathrm{E} 2}$} & $2.56 \mathrm{~m}^{3}$ vapour \\
\hline \multirow[t]{2}{*}{ Product pump } & \multirow{2}{*}{$\begin{array}{l}P_{i}=147.1 \mathrm{kPa} \\
P_{o}=312.8 \mathrm{kPa} \\
37 \mathrm{~kg} / \mathrm{min} \text { water } \\
4.1 \mathrm{~kg} / \mathrm{min} \text { solute }\end{array}$} & & $\begin{array}{l}3.25 \mathrm{~m}^{3} \text { liquor } \\
82.1^{\circ} \mathrm{C} \\
50.0 \mathrm{kPa}\end{array}$ \\
\hline & & \multirow[t]{2}{*}{$V V 3$} & $P_{o}=45 \mathrm{kPa}$ \\
\hline$L V 3$ & $\begin{array}{l}P_{o}=250 \mathrm{kPa} \\
C_{v}^{2}=27.0\end{array}$ & & $\begin{array}{l}C_{v}^{2}=304.5 \\
39.0 \mathrm{~kg} / \mathrm{min}\end{array}$ \\
\hline & $P_{0}$ o & ent & \\
\hline
\end{tabular}

Table 6.2: Optimum steady state variable summary

chosen as inputs of the linear system, and the measured variables, constraints slacks and objective function were chosen as outputs, yielding a linear system appropriate for MILP analysis of control structures.

The next step is costing the manipulated and measured variables for use in the MILP. As the process has been optimised based on annual operating profits, it will be necessary to annualise the installation costs of the control equipment. It will be assumed that maintenance of control equipment will increase its cost by $50 \%$ of the annualised installed cost. The plant is expected to run for 15 years at an interest rate $i$ of $20 \%$ for time value of money calculations. The annualised $\operatorname{cost} C_{A}$ for equipment with an installed cost of $C_{I}$ is given by (assuming first payment at the end of year 1 and no scrap value):

$$
C_{I}=C_{A} \sum_{n=1}^{15} \frac{1}{(1+i)^{n}}=4.675 C_{A}
$$

The total annualised cost $C_{A}^{\prime}$ of sensors and valves is given by $C_{A}^{\prime}=1.5 \times C_{A}$ 


\begin{tabular}{|l|l|}
\hline Constraint & Lagrange multiplier \\
\hline E1 liquor volume lower bound & $0.0 £ / \mathrm{m}^{3}$ \\
E2 liquor volume lower bound & $0.0 £ / \mathrm{m}^{3}$ \\
Product concentration & $71,430 £ /($ weight fraction) \\
$V V 1$ pressure drop & $5,880 £ / \mathrm{kPa}$ \\
$V V 2$ pressure drop & $8,820 £ / \mathrm{kPa}$ \\
$V V 3$ pressure drop & $12,200 £ / \mathrm{kPa}$ \\
\hline
\end{tabular}

Table 6.3: Steady state active constraint summary

E1 steam feed rate

E1 steam chest temperature

E1 pressure

E1 liquor volume

E2 steam chest pressure

E2 temperature

E2 outlet vapour rate

E2 freeboard liquor head

LV2 liquor flowrate

$L V 2$ solute flowrate

$L V 3$ liquor flowrate

$L V 3$ solute flowrate
E1 steam chest pressure

E1 temperature

E1 outlet vapour rate

E1 freeboard liquor head

E2 steam chest temperature

E2 pressure

E2 liquor volume

$L V 2$ water flowrate

$L V 2$ liquor composition

$L V 3$ water flowrate

$L V 3$ liquor composition

Table 6.4: Potential measured variables on the double effect evaporator 


\begin{tabular}{|l|c|}
\hline Variable & Installed cost $£$ \\
\hline Valve & 2,000 \\
Liquor flow & 2,000 \\
Vapour flow & 3,000 \\
Temperature & 3,000 \\
Pressure & 4,000 \\
Volume/head & 4,000 \\
Composition & 5,000 \\
Component flow & 7,000 \\
\hline
\end{tabular}

Table 6.5: Measurement/valve costs for double effect evaporator

(maintenance costs).

$$
C_{A}^{\prime}=\frac{3}{2} \frac{C_{I}}{4.675}
$$

or

$$
C_{A}^{\prime} \approx 0.32 C_{I}
$$

The next step is to assign appropriate costs for installation of measurement equipment and control valves. The cost assignments are summarised in table 6.5. The composition measurement cost is based on the assumption that accurate compositions can be readily calculated from the refractive index of the liquor. Component flow measurement cost is assumed to be the sum of composition and flow measurement costs.

The last step before solving the MILP is assigning appropriate disturbance magnitudes and frequencies. Three disturbance cases were considered for this study: a base case disturbance; a case considering the benefits of reducing the steam variability before it reaches the plant; and a multiple disturbance frequency analysis considering process disturbances ranging from noise to diurnal temperature variation. For this plant the most common disturbance occurs at a period of approximately three hours $(\omega \approx 0.005$ cycles $/ \mathrm{min})$. This frequency is used as the nominal disturbance frequency for all cases. The disturbance magnitudes are discussed in the analysis of each case below.

Prior to examining each case in detail, it was noted that no feasible open loop solution to the problem was found for any of the disturbance cases. This does not necessarily imply that there is no open loop plant physically possible. It could 
be that the open loop plant needs to be operated sufficiently far from the optimum steady state operating point that the linearisation is invalid. A significant benefit of the control study is to suggest control structures which allow operation near the steady state optimum.

\subsubsection{Case 1 - Base case disturbance}

The disturbance magnitudes are given in table 6.6, based on upstream and downstream processing, and utility variation (the variation in steam temperature corresponds to $2 \%$ variation in the steam pressure).

\begin{tabular}{|l|l|}
\hline Disturbance & Magnitude \\
\hline Feed pressure & $10 \mathrm{kPa}$ \\
Feed temperature & $5{ }^{\circ} \mathrm{C}$ \\
Feed composition & $0.35 \%$ \\
Product liquor pressure & $5 \mathrm{kPa}$ \\
Steam temperature & $0.5^{\circ} \mathrm{C}$ \\
\hline
\end{tabular}

Table 6.6: Base case disturbance at $\omega=0.005$ cycles $/ \mathrm{min}$

Given this information, the MILP was solved for the five best control structures, summarised in table 6.7. $T$ and $P$ are the temperature and pressure of the liquor side in the second effect, $X$ and $F_{t}$ are the product liquor composition and total flow.

\begin{tabular}{|cc|c|}
\hline Inputs & Outputs & Cost $£$ \\
\hline$V V 3, L V 2$ & $T, P$ & 8,960 \\
$V V 3, L V 2$ & $T, X$ & 9,280 \\
$V V 3, L V 2$ & $P, X$ & 9,600 \\
$V V 3, L V 2, L V 3$ & $T, P, F_{t}$ & 10,240 \\
$V V 3, L V 2, L V 3$ & $T, F_{t}, X$ & 10,560 \\
\hline
\end{tabular}

Table 6.7: Optimal control structures for disturbance case 1

The first three control structures consist of the vapour product valve $V V 3$ and the intermediate liquor valve $L V 2$ being used to control either effect 2 temperature and pressure or effect 2 temperature and product composition or effect 2 pressure and product composition. All three of these control structures are seeking 
to operate on the product composition constraint and the $V V 3$ pressure drop constraint. This occurs because of the vapour-liquid equilibrium effects, where specifying any two of pressure, temperature and composition automatically fixes the third. Thus the three control structures will operate the plant at the same steady state conditions, with the only differences in economics being the costs of the sensors. By examining the Lagrange multipliers of the constraints (table 6.3), it can be seen that these control structures correspond to a "good" set of control objectives. The Lagrange multiplier of the constraints are $£ 71,400 /$ weight fraction for composition and $£ 12,200 / \mathrm{kPa}$ for $V V 3$ pressure drop, with the next largest multiplier $£ 8,800 / \mathrm{kPa}$ on $V V 2$ pressure drop. Clearly the MILP has selected the measurements which allow control at the constraints with the largest effect on process economics. From this it can be deduced that the return from controlling the pressure drop $V V 3$ and the product composition justified the cost of installation of control loops, but controlling the pressure drop over $V V 2$ does not return sufficient benefit to justify installing a control loop. Note that although the liquor holdups have increased to allow for dynamic process variation, they have no effect on the process economics, as working capital has been ignored for this problem.

As discussed in chapter 4 , the linear dynamic economic analysis requires some controllability analysis to test the validity of the perfect control assumption. Therefore $l_{\infty}$ minimum condition number plots were generated for each control structure (figure 6.2). The condition numbers are plotted over a frequency range of 1 cycle per second (process noise) to 1 cycle per day (diurnal variation). Additionally the transmission zeros for all of the control structures were calculated to check for right half plane zeros (RHPZ). Only the $V V 3, L V 2, L V 3, T, P, F_{t}$ structure contained a RHPZ at $1.1 \times 10^{-6}$, and as this structure was ranked fourth by economics, it was not examined any further. As the three best economic control structures have no RHP zeros, non minimum phase behaviour is not expected. However, examining the condition number plots it can be seen that for the best economic structure $V V 3, L V 2, T, P$, the minimum condition number varies from 2 at low frequency to 100 at high frequency, suggesting that it will be difficult to achieve tight control of the process subject to high frequency disturbances (process noise). The second best configuration $V V 3, L V 2, T, X$ would achieve significantly better control at high frequencies, with a maximum condition number of 1.5 over the frequency range. The 
difference in cost between the two control configurations is $£ 320$ per annum - the cost difference between a pressure sensor and a composition sensor. In this case the design engineer would be able to make a decision that for the benefits of improved controllability, the second control configuration is significantly better, as it will reject disturbances well across the entire frequency range for a minimal increase in cost, and as it has a minimum condition number close to 1 , it should possible to achieve very near to perfect control.

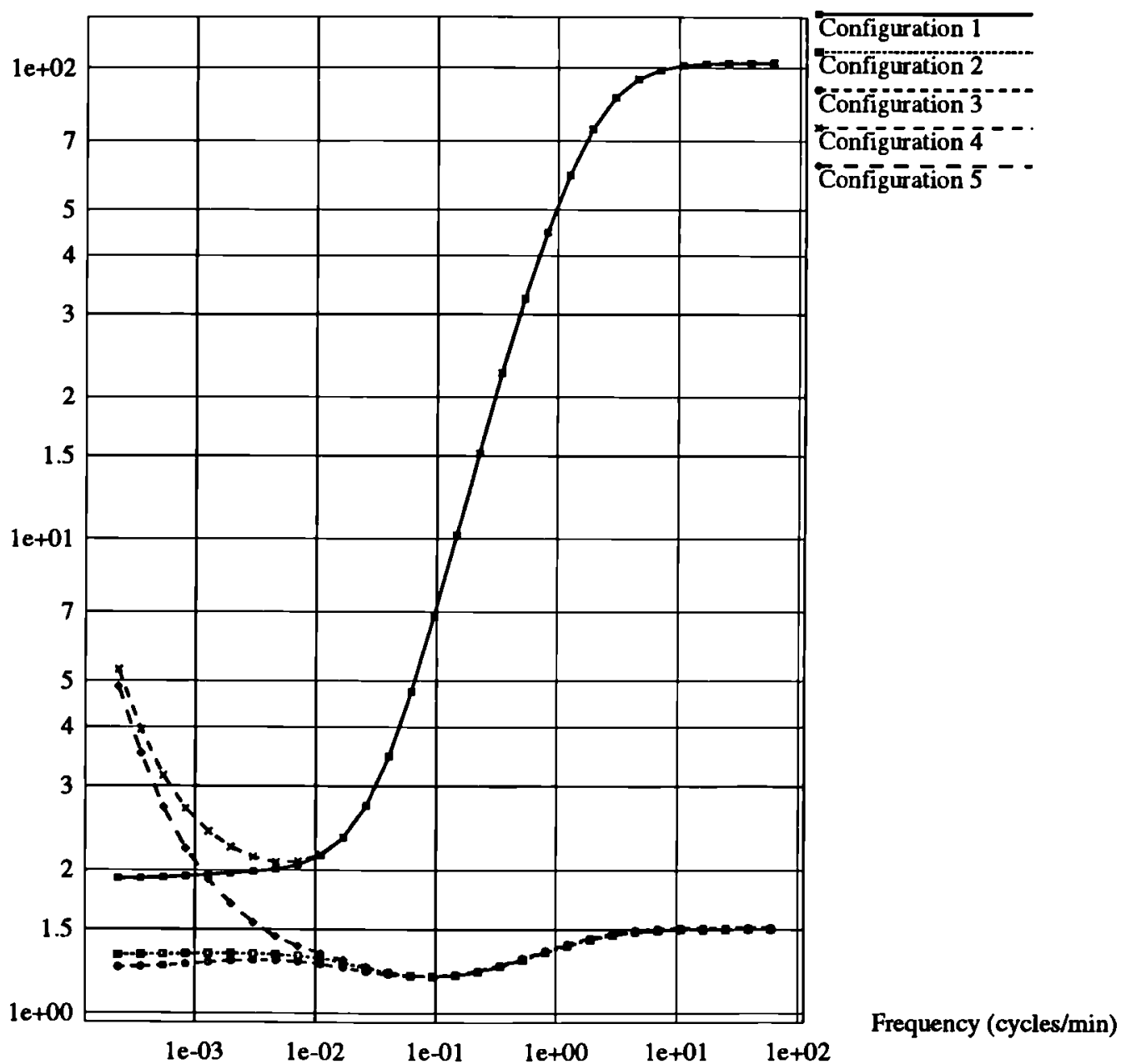

Figure 6.2: $l_{\infty}$ minimum condition number plot for disturbance case 1 


\subsubsection{Case 2 - Reduced steam variability}

This case is a benefits analysis considering a trade off between increased steam utility costs and reduced steam variability, using the MILP analysis to assess the expected improvement in plant economics. It is estimated that a reduction in the base case steam variability from $0.5^{\circ} \mathrm{C}$ to $0.25^{\circ} \mathrm{C}$ will cost $£ 500$ per annum, and we want to determine if the improvement in plant performance is sufficient to justify the investment. The disturbance magnitudes are given in table 6.8 and the five best structures are summarised in table 6.9. The condition number plot is similar to figure 6.2 , but as it has no bearing on the following discussion, it is included in appendix $\mathrm{D}$ for reference. The $V V 3, L V 2, L V 3, T, P, F_{t}$ structure contained the only RHPZ and again it was ignored as it was ranked fourth in the economics.

\begin{tabular}{|l|l|}
\hline Disturbance & Magnitude \\
\hline Feed pressure & $10 \mathrm{kPa}$ \\
Feed temperature & $5{ }^{\circ} \mathrm{C}$ \\
Feed composition & $0.35 \%$ \\
Product liquor pressure & $5 \mathrm{kPa}$ \\
Steam temperature & $0.25^{\circ} \mathrm{C}$ \\
\hline
\end{tabular}

Table 6.8: Case 2 disturbance at $\omega=0.005$ cycles $/ \mathrm{min}$

\begin{tabular}{|cc|c|}
\hline Inputs & Outputs & Cost $£$ \\
\hline$V V 3, L V 2$ & $T, P$ & 7,460 \\
$V V 3, L V 2$ & $T, X$ & 7,780 \\
$V V 3, L V 2$ & $P, X$ & 8,100 \\
$V V 3, L V 2, L V 3$ & $T, P, F_{t}$ & 8,740 \\
$L V 2$ & $X$ & 8,910 \\
\hline
\end{tabular}

Table 6.9: Optimal control structures for disturbance case 2

The first four structures for this case are identical to the base case, and the economics are similar. Therefore the same conclusion may be drawn: The most appropriate control structure is the $V V 3, L V 2, T, X$ structure. Considering the benefits analysis, comparing the economics of the base case and case 2 ( $V V 3, L V 2, T, X$ structure) yields an improvement of $£ 1,500$ per annum for the reduced variability. This implies an estimated improvement in performance of $£ 1,000$ per annum. Note 
that the improvement is only estimated as the economic analysis is based on perfect control. However as the best control structures are identical for both disturbance cases, the performance degradation of an implemented process controller compared to perfect control would be expected to be similar for both cases. Therefore the estimate of $£ 1,000$ per annum should be relatively accurate, suggesting it is worth investing in reduced steam variability. This case demonstrates that the control structure analysis can be used to assess benefits of reducing plant variability in a relatively simple manner, provided some cost information for disturbance reduction is available (e.g. costs of increased feed and intermediate storage for buffering).

\subsubsection{Case 3 - Multiple disturbance frequencies}

The final case considers multiple disturbance frequencies, with disturbances ranging from 3 second process noise to diurnal variations in process temperatures. The multiple frequency analysis is implemented as outlined in the previous chapter using the three hour disturbance as the base case, with the disturbances summarised in table 6.10. Note that the vapour pressure exiting the second effect is added as a high frequency disturbance. The first 10 structures are summarised in table 6.11, where $F_{w}$ and $F_{s}$ are the water and solute product component flows. Again the condition number plot of the first five structures is included in appendix $D$ for reference. None of the structures contain RHPZs.

\begin{tabular}{|l|c|c|c|c|c|l|}
\hline Variable & \multicolumn{5}{|c|}{ Disturbance Period } & \multirow{2}{*}{ Units } \\
\cline { 2 - 6 } & 3 second & 1 minute & hourly & 3 hourly & diurnal & \\
\hline Feed pressure & 0.5 & 1.5 & & 10 & & $\mathrm{kPa}$ \\
Feed temperature & & & 3 & 5 & 5 & ${ }^{\circ} \mathrm{C}$ \\
Feed composition & & & 0.1 & 0.35 & & $\%$ \\
Steam temperature & & 0.25 & 0.25 & 0.25 & 0.5 & ${ }^{\circ} \mathrm{C}$ \\
Product pressure & 0.5 & 1.5 & & 5 & & $\mathrm{kPa}$ \\
VV3 outlet pressure & 0.5 & 0.5 & & & & $\mathrm{kPa}$ \\
\hline
\end{tabular}

Table 6.10: Summary of evaporator disturbances

An interesting result occurs when considering the multiple disturbance frequency case: the optimal control structure changes from 2 by 2 to the SISO structure $L V 2, X$. This is an effect of the E2 vapour outlet pressure disturbance, which is not 


\begin{tabular}{|cc|c|}
\hline Inputs & Outputs & Cost $£$ \\
\hline$L V 2$ & $X$ & 10,510 \\
$L V 2, L V 3$ & $F_{t}, X$ & 11,790 \\
$L V 2, L V 3$ & $F_{s}, F_{t}$ & 12,430 \\
$L V 2, L V 3$ & $F_{w}, F_{t}$ & 12,430 \\
$L V 2, L V 3$ & $F_{s}, X$ & 13,390 \\
$L V 2, L V 3$ & $F_{w}, X$ & 13,390 \\
$L V 2, L V 3$ & $F_{s}, F_{w}$ & 14,030 \\
$V V 3, L V 2$ & $T, P$ & 14,560 \\
$V V 3, L V 2$ & $T, X$ & 14,880 \\
$L V 2, L V 3$ & $P, X$ & 15,200 \\
\hline
\end{tabular}

Table 6.11: Optimal control structures for multiple disturbance frequencies

present in the single disturbance frequency cases. This effect may be seen by examining the $V V 3, L V 2, T, X$ (dual control) and $L V 2, X$ (composition control) structures under the base case disturbance (3 hour disturbance in table 6.10) and an augmented disturbance consisting of the base case and an additional $0.5 \mathrm{kPa}$ variation in the outlet pressure of $V V 3$. The costs of each case are given in table 6.12. Considering the effects of the controllers for the base case, the dual controller holds the slack variables of the composition constraint and the $V V 3$ pressure drop constraint at zero (i.e. no deviation from the constraints), and thus has improved economics over the composition control case which only maintains the composition constraint slack variable at zero. In the augmented disturbance case, the dual control again controls the pressure inside the effect with no deviation. However the exit pressure of $V V 3$ is varying by $\pm 0.5 \mathrm{kPa}$. Therefore, in order to ensure that a $V V 3$ pressure drop of at least $5.0 \mathrm{kPa}$ occurs at all times, the second effect must be operated at least $5.5 \mathrm{kPa}$ above the nominal $V V 3$ exit pressure (as the effect is operating at constant pressure). This corresponds to a $0.5 \mathrm{kPa}$ step away from the pressure drop constraint. The effect of this step is calculated as the product of the step multiplied by the constraint Lagrange multiplier $(0.5 \times 12200=£ 6,100)$ or the difference between the base case and augmented case dual control. The performance of the composition control strategy improves relative to dual control for the augmented disturbance by allowing the effect pressure to float at a cost of slightly increased giveaways on all of the vapour line valve pressure drops. From this it can be concluded that the 
control structure is strongly dependent on the magnitude and the structure of the disturbances.

\begin{tabular}{|l|c|c|}
\hline Structure & \multicolumn{2}{|c|}{ Costs $£$} \\
& Base case & Augmented Case \\
\hline Dual & 7,780 & 13,880 \\
Composition & 8,910 & 11,160 \\
\hline
\end{tabular}

Table 6.12: Cost comparison of dual and composition control

This result raises a cautionary point about the use of the MILP analysis with respect to the interaction between disturbance frequencies and operating constraints. In practice, violation of operating constraints by high frequency, low magnitude disturbances (noise) will often be acceptable for normal process operation. In this case, no practical benefit results from examining process noise as one would expect to add a filter and ignore it. In the above case, dual control would remain preferable to the composition control as it has the more profitable base case economics if the $V V 3$ outlet pressure noise is regarded as negligible. A similar argument may be posed for low frequency disturbances, where it would be expected to modify the plant operating point by steady state operating optimisation, rather than continuing suboptimal operation.

From this it can be concluded that the economic disturbance analysis should only consider those disturbance frequencies which are expected to have a significant impact on the dynamic plant operation, while noise and low frequency effects should be assumed to be handled either by appropriate filtering or steady state optimisation. The issue raised here is that both the disturbance and constraint sets must be characterised carefully to represent the expected modes of plant operation, and the economic analysis should be carried out over the frequency range using the constraint/disturbance characterisation. For instance, cases may occur where only a subset of the constraints must be satisfied at all frequencies, in which case the economic analysis should be carried out all frequencies, using the constraint subset and any other constraints as appropriate (for example the remaining base case constraints for the nominal disturbance frequency). 


\subsubsection{Computational results}

For the two base cases, it would require about 70,000 Sparc II cpu seconds for explicit enumeration of all possible solutions (i.e. solution of the perfect control subproblem for each potential control structure). The optimal solutions were found in 4,600 to 5,000 cpu seconds using the MILP method, and the five best solutions were found within 8,500 to $9,900 \mathrm{cpu}$ seconds, resulting in selection of the optimal (first) control structure approximately 15 times faster than by explicit enumeration. This is a significant speed up, but further improvements should be possible by tightening the problem formulation. Performance issues will be discussed in greater depth in the next case study.

For the multiple disturbance frequency case, the optimal solution was found within $16,500 \mathrm{cpu}$ seconds, and the five best solutions within $28,000 \mathrm{cpu}$ seconds. The reason for the relatively slow solution time for the multiple disturbance frequency case is due to the solution method, where a control structure is generated using a base case disturbance, and then the control structure is evaluated using the full disturbance set. The base case economics underestimate the actual multiple frequency economics dramatically, and therefore many more control structures must be examined to ensure optimality. This suggests that a more appropriate method for solving the multiple frequency case would be to pose the MILP master problem as a multiperiod problem, with an LP representing the plant at each frequency, and the integer variables as common variables between the different periods (frequencies).

\subsubsection{Section summary}

This case study demonstrated that the MILP analysis can be used to generate economically optimal control structures rapidly, but that there is still scope to improve the MILP performance by tightening the LP representation. The need to use controllability indicators to examine the generated control structures was emphasised by the fact that the economically optimal control structure had poor controllability characteristics at high frequency, whereas an alternative control structure had significantly better controllability properties over the entire frequency range for a minimal increase in cost. The MILP analysis demonstrated a sensible selection of economic control objectives, choosing to minimise the variation of the most 
economically significant constraints using the cheapest sensors available.

It was also demonstrated that the control structure analysis can be used to assess the benefits of reducing or eliminating process disturbances before they affect the plant. This suggests potential employment of the method for sizing plant buffer capacities.

Finally, from examination of the multiple frequency disturbance case, it was concluded that interactions between disturbance frequencies/magnitudes and operating constraints must be characterised carefully to ensure that noise and steady state disturbances are not overemphasised in the structure selection problem. The results of the multiple frequency analysis suggested the speed of solution could be improved by treating the problem as a multiperiod MILP.

\subsection{Mount Isa Mines froth flotation circuit}

This case study is based on the Mount Isa Mines froth flotation circuit studied by Fewings, Slaughter, Manlapig and Lynch (1979), who considered the design of a process control system for the circuit. This case study considers the problem of designing the circuit for optimal steady state and dynamic performance. The simplifications used in developing the case study as a design problem result in a process which does not reflect all of the properties of the system studied by Fewings et al., but does highlight aspects of the MILP control structure selection algorithm.

The froth flotation circuit is used to concentrate $5,000,000$ tonnes per annum of a $3.2 \%$ copper chalcopyrite ore. Figure 6.3 summarises the process flowsheet. The process is fed from a wet grinding circuit, with collector added to the circuit feed to improve the flotation characteristics of the valuable material. There are five flotation banks: rougher; scavenger; cleaner; recleaner and retreater. The banks typically consist of four to twelve flotation cells. Each flotation bank has an adjustable aeration rate, that sets the rate for all cells in the bank. Makeup water may be added at the entrance to each bank to ensure acceptable slurry flow. All cell tailings flows are set by valves on the tailings lines. The regrind mill serves to reduce the fraction of heavier particulate material, and thus improve the mineral flotation characteristics. The process objective is to minimise copper loss, while producing a concentrate at a minimum acceptable grade for downstream processing (similar to 
the flotation circuits discussed in chapter 4).

The process flowsheet is a simplification of the actual circuit, which includes further reagents to improve the process performance, such as depressants and frothers, and additional process units such as hydrocyclones employed in the process. Fewings et al. (1979) developed a process model of the plant based only on the units and variables which have a significant impact on the steady state and dynamic process behaviour. For modelling purposes, the flotation banks were lumped as either three cell (rougher, scavenger and cleaner) or single cell (retreater, recleaner) banks. In the three cell banks, the cells are connected in series by the tailings streams, the bank tailings stream is the tailings of the final cell, and the bank concentrate is the combination of the three cell concentrates. A nonlinear dynamic model of the process appropriate for use in this project has been developed by Barton and Perkins (1988) from the initial work of Fewings et al. The flotation model treats the pulp phase to froth phase flotation mechanism as independent first order reactions for each of the five components: fast floating valuables; slow floating valuables; fast floating gangue; slow floating gangue; and water (similar to the Chan et al. (1991) model). The collector addition is modelled as changing the proportion of fast and slow floating valuables, and the regrind mill is modelled as converting the slow floating valuables to fast floating valuables. For this case study, the control loops used by Barton and Perkins were ignored. For further discussion of the process behaviour, refer to Fewings et al. (1979). Two minor modifications were required in order to use the Barton and Perkins model for this case study, both of which are summarised here and are detailed in appendix E. Firstly the model was modified to include the copper assay (weight percentage copper in the valuables (Fewings et $a l$., 1979)) for use in the objective function, and secondly the model was modified to handle the effect of collector more realistically. Initially the collector addition defined the proportion of fast and slow floating valuables in the feed. With the changes to the model, the collector addition may only modify the proportions of the valuable components as fraction of the valuable components in the feed. Fewings et al. also showed that the collector could only achieve a limited improvement in the process performance. Based on this, the collector effectiveness was limited to increasing the quantity of fast floating valuables by up to $20 \%$ of the total valuables (with a corresponding decrease in slow floating valuables). The 


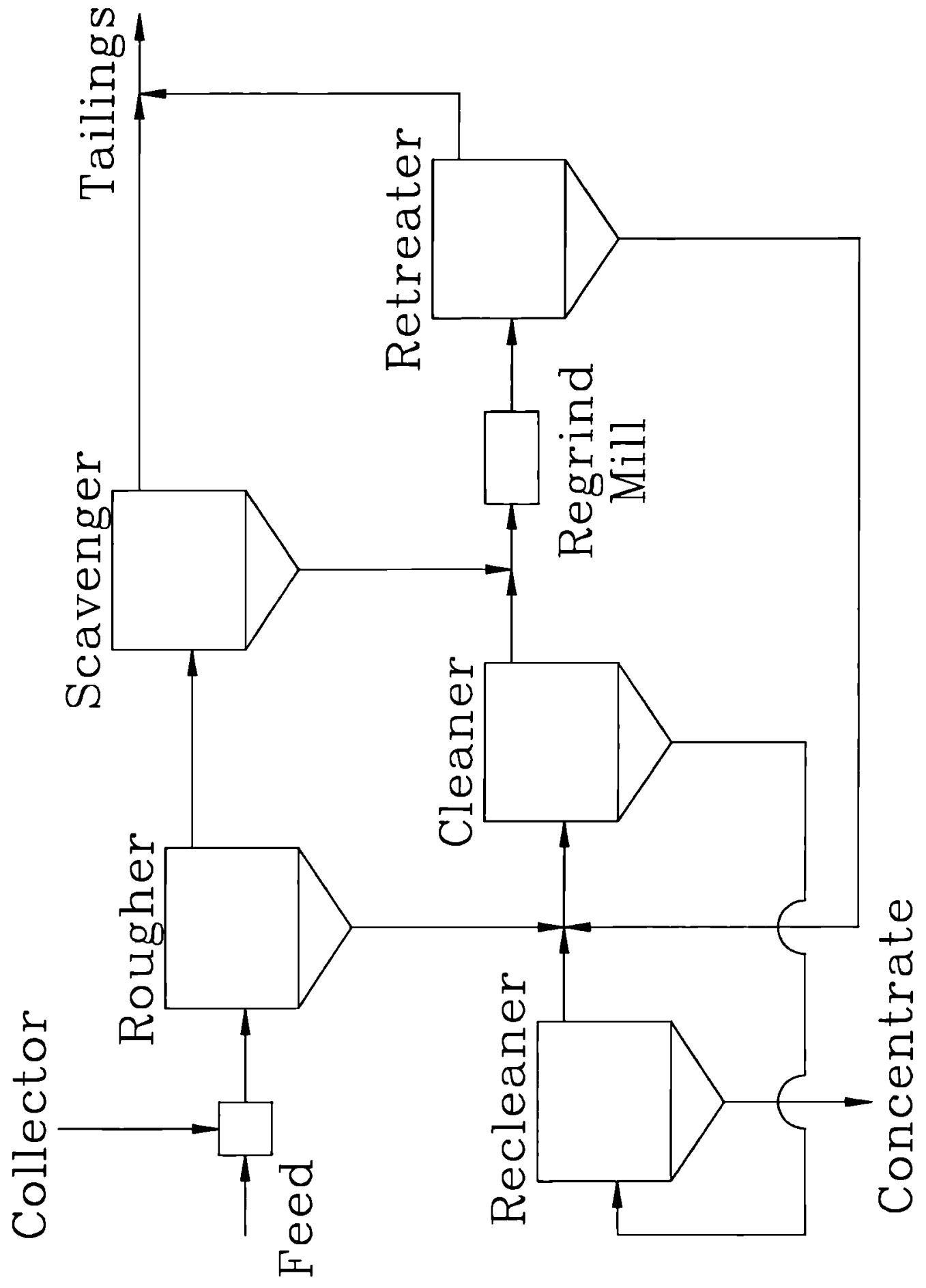

Figure 6.3: Mount Isa Mines froth flotation circuit 
models and the modifications are further discussed in appendix $\mathbf{E}$.

The next step for the design problem was to pose the steady state optimisation. As this problem includes capital costs, it was decided to use a cash flow discounted to year 0 . The plant is to be run for 15 years at 8000 hours per year, with an assumed interest rate of $20 \%$. The exchange rate for converting US $\$$ to $£$ is taken as 1.7. The Marshall and Swift indices are taken from the appropriate issues of Chemical Engineering. The objective function used was of the form:

Minimise $\{$ Value of copper lost in tailing + Production costs + Capital costs

The use of the copper loss as a measure of productivity warrants brief discussion. The copper loss in the tailings is not processed in the smelter, and thus represents loss of revenue. Therefore it is appropriate to balance the potential value of copper in the waste stream against the processing costs. Note that it is only a potential value, as no smelter process is able to recover $100 \%$ of the metal in an ore, but it still provides a useful measure of process losses for use in the objective function. Each term in the objective function will now be expanded and defined.

The copper value term is simply given by the total copper loss in the process tailings stream multiplied by the value of the copper. Using a value of $£ 1734$ per tonne (Sarjeantson, 1990) for the copper results in the annual copper loss term: $8000 \times 1734 T_{v} A_{T} . T_{v}$ is the tailings valuables in tonnes per hour, and $A_{T}$ is the tailing copper assay (mass fraction copper).

The capital costs of the process consist of the cost of the froth flotation cells and the regrind mill. For this problem it was assumed that the regrind mill had a fixed capital cost, regardless of throughput, and therefore does not affect the optimisation. It was assumed that cells were available off the shelf in the size range 20 to $80 \mathrm{~m}^{3}$ (noting that the cells used in the model are actually a lumped series of cells), and that the cell costs could be approximated to that of a stirred tank reactor, with an additional multiplier of 5 to allow for ancillary equipment (pumps, settlers, cyclones) and mineral processing overheads. The base cost for a cell was developed using the Guthrie correlation for installed cost of a stirred tank reactor (Douglas, 1988). The 1991 Marshall and Swift index of 925.9 was used, along with a construction factor of 1.0. Assuming that the cell height to diameter ratio is one, 
and after appropriate manipulation, the cost per cell is given by:

$$
£ 23645 \times V^{0.6227}
$$

where $V$ is the cell volume in $\mathrm{m}^{3}$. It was assumed that the salvage value of the plant was zero and the plant working capital was negligible.

For the production costs, reagents such as depressants, $\mathrm{pH}$ conditioners, frothers and flocculants are assumed to be added at a constant rate based on the nominal plant feeds, and are therefore fixed costs which do not affect the optimisation. Similar arguments may be posed for the cost of labour and buildings. It is also assumed that the variation in power consumption with production is negligible. The remaining operating costs are: grinding steel; water usage (makeup only); and collector costs. The water costs are relatively simple, as the plant is charged for all excess water used in the plant (assuming no recycle) at a cost of US\$ 0.03/1000 gallons (Douglas, 1988). The grinding costs are based on a figure of 1.5 pounds of steel per tonne of ore ground (Fuerstenau, 1962), at a cost of US $\$ 0.33 / \mathrm{kg}$ for wire rod (Sarjeantson, 1990), giving a yearly cost of $1056 \times$ solids grinding rate pounds per annum. The collector required a certain amount of extrapolation from the available literature. For this case it was assumed that a $10 \%$ change in valuables flows (see above discussion of collector model) corresponds to a collector addition rate of 1 pound per tonne of solids (an average figure for collector addition (Kirk-Othmer, 1985)). It was also assumed that the collector effect varied linearly with collector addition (Fewings et al., 1979). That is, a $20 \%$ change corresponds to 2 pounds collector per tonne of solids. A collector cost of 1960 US\$0.0397/pound (Kirk-Othmer, 1985) was used, along with the 1960 Marshall and Swift index of 240 to give a collector cost of $72 \times$ collector improvement percent $\times$ feed solids flow. Unless otherwise stated, all flows have been assumed to be tonnes/hour.

The above information, along with a discount factor of 4.67547 over 15 years can be be used to write a simple objective function. The optimisation problem also requires constraints and the identification of the free optimisation variables. The constraints imposed on the process were:

1. The circuit concentrate must have a grade (mass percentage of copper in the solids) of at least $20 \%$ 
2. All streams in the process must contain at least $50 \%$ by mass water for acceptable slurry flow

3. The pulp volumes of all cells must lie within the available range of 20 to $80 \mathrm{~m}^{3}$

4. The valve coefficients for tailings flows must lie within the range 0 to 1500 for the first and second cells in multicell banks, and 0 to 150 for the last cell in multicell banks and single cell banks

5. The aeration rate for each cell must lie in the range 0.5 to 1.5

The 11 tailings valve coefficients, the 5 aeration rates, the 5 makeup water flows, and the collector addition rate were identified as free optimisation variables. The nominal feed conditions for the plant are given in table 6.13 (Fewings et al., 1979).

\begin{tabular}{|l|c|}
\hline Fast floating valuables & 20 tonnes/hour \\
Slow floating valuables & 20 tonnes/hour \\
Fast floating gangue & 4 tonnes/hour \\
Slow floating gangue & 316 tonnes/hour \\
Water & 720 tonnes/hour \\
Copper assay & $32 \%$ \\
\hline
\end{tabular}

Table 6.13: Nominal operating conditions for MIM circuit

The optimum process design resulted in a minimum processing cost of $£ 19,834,000$ discounted to year 0 . A brief summary of the optimum steady state operating conditions is given in table 6.14, with a full breakdown in appendix $\mathrm{E}$. A constraint summary is given in table 6.15. As is expected in a process of this nature, the optimisation is equipment constrained rather than process limited, as froth flotation is a cheap concentration operation for a high value added product. That is, the optimum is limited by the available equipment sizes (flotation cell volumes), rather than the physical concentration mechanisms.

Six potential disturbances to the process were identified: the five component feeds and the feed copper assay. It was decided not to use the copper assay, as the proportion of metal in the valuable component varies slowly, and is more appropriately treated as an optimising control disturbance. As mineral processing 


\begin{tabular}{|l|c|l|c|}
\hline Rougher cell 1 $K_{v}$ & 678 & Rougher cell 2 $K_{v}$ & 660 \\
Rougher cell 3 $K_{v}$ & 53.8 & Scavenger cell 1 $K_{v}$ & 580 \\
Scavenger cell 2 $K_{v}$ & 561 & Scavenger cell 3 $K_{v}$ & 60.3 \\
Cleaner cell 1 $K_{v}$ & 1500 & Cleaner cell 2 $K_{v}$ & 1025 \\
Cleaner cell 3 $K_{v}$ & 73.0 & Retreater $K_{v}$ & 55.9 \\
Recleaner $K_{v}$ & 88.2 & & \\
Rougher aeration rate & 1.5 & Scavenger aeration rate & 1.5 \\
Cleaner aeration rate & 1.5 & Retreater aeration rate & 1.5 \\
Recleaner aeration rate & 1.5 & & \\
Rougher makeup water & 0 & Scavenger makeup water & $50.5 \mathrm{t} / \mathrm{h}$ \\
Cleaner makeup water & 0 & Retreater makeup water & 0 \\
Recleaner makeup water & 0 & & \\
\hline
\end{tabular}

Table 6.14: Optimal steady state parameters of MIM circuit

plants are subject to frequent upsets as different pockets are mined and due to the variability of upstream processing, a disturbance frequency of 1 cycle per hour was used for the MILP analysis (corresponding closely to the dominant time constant of the process). The disturbance magnitudes are summarised in table 6.16. An open loop linear disturbance analysis was carried out, and the economic giveaway was estimated at $£ 1,200,000$ or roughly $6 \%$ of the cost of the plant, indicating a useful potential improvement in economics through addition of a control system.

The next step was identification and costing of potential measured and manipulated variables. In the initial examination of the plant, the 22 free optimisation variables were identified as potential manipulated variables, along with 66 potential measured variables. However the resulting problem (with several billion potential control structures), proved too slow to solve with the current MILP implementation. Therefore the problem was pared down to more closely resemble the set of measurements and manipulated variables available in the Fewings et al case study. This resulted in a problem with 11 potential manipulated variables and 19 potential measured variables which are summarised in table 6.17. This problem has ${ }^{30} C_{11} \approx 54,000,000$ potential control structures. The problem also initially included linearisations of all 45 constraints used in the steady state model. However, this also slowed the problem solution significantly, and as a result a reduced constraint set was employed based on those constraints which are either active or near active at the nominal steady state optimum, which are summarised in table 6.15. 


\begin{tabular}{|l|c|c|}
\hline Constraint & Slack & $\begin{array}{c}\text { Lagrange multiplier } \\
(£ 1,000 \mathrm{~s})\end{array}$ \\
\hline Product grade & 0.0 & $1177 / \%$ copper \\
Rougher cell 1 volume upper bound & 0.0 & $2.55 / \mathrm{m}^{3}$ \\
Rougher cell 2 volume upper bound & $3.646 \mathrm{~m}^{3}$ & 0.0 \\
Cleaner cell 1 volume upper bound & 0.0 & $71.58 / \mathrm{m}^{3}$ \\
Cleaner cell 1 volume upper bound & $0.421 \mathrm{~m}^{3}$ & 0.0 \\
Cleaner cell 1 volume upper bound & $1.524 \mathrm{~m}^{3}$ & 0.0 \\
Retreater volume upper bound & 0.0 & $34.19 / \mathrm{m}^{3}$ \\
Scavenger tailings minimum water & 0.0 & $21.3 /$ tonne water \\
Recleaner tailings minimum water & 39.4 tonne & 0.0 \\
Retreater tailings minimum water & 165.9 tonne & 0.0 \\
Rougher aeration upper bound & 0.0 & $578 /$ air factor \\
Scavenger aeration upper bound & 0.0 & $331 /$ air factor \\
Cleaner aeration upper bound & 0.0 & $4242 /$ air factor \\
Recleaner aeration upper bound & 0.0 & $105 /$ air factor \\
Retreater aeration upper bound & 0.0 & $1974 /$ air factor \\
Collector upper bound & 0.0 & $238 / \%$ change \\
Cleaner cell 1 tailings valve & 0.0 & 0.032 tonne $e^{1 / 2} /$ hour \\
\hline
\end{tabular}

Table 6.15: MIM steady state constraint summary

The final results were then tested to ensure that they satisfied the full constraint set. As a final note, it is assumed that there is sufficient freeboard in the cells to absorb uncontrolled variation in the pulp phase volume, unless the cells volumes are on their upper or lower design limits. That is, the cells are designed to allow for pulp volume variation. This implies that the cell volumes will only be controlled if there is an economic incentive. Note also that as the cells are controlled by square root law valves, they will tend to be self regulating in practice.

\begin{tabular}{|l|c|}
\hline Variable & $\begin{array}{c}\text { Magnitude } \\
\text { (tonnes/hour) }\end{array}$ \\
\hline Water & 72 \\
Fast floating valuables & 2 \\
Slow floating valuables & 2 \\
Fast floating gangue & 1 \\
Slow floating gangue & 31 \\
\hline
\end{tabular}

Table 6.16: Disturbance magnitudes for MIM case study 


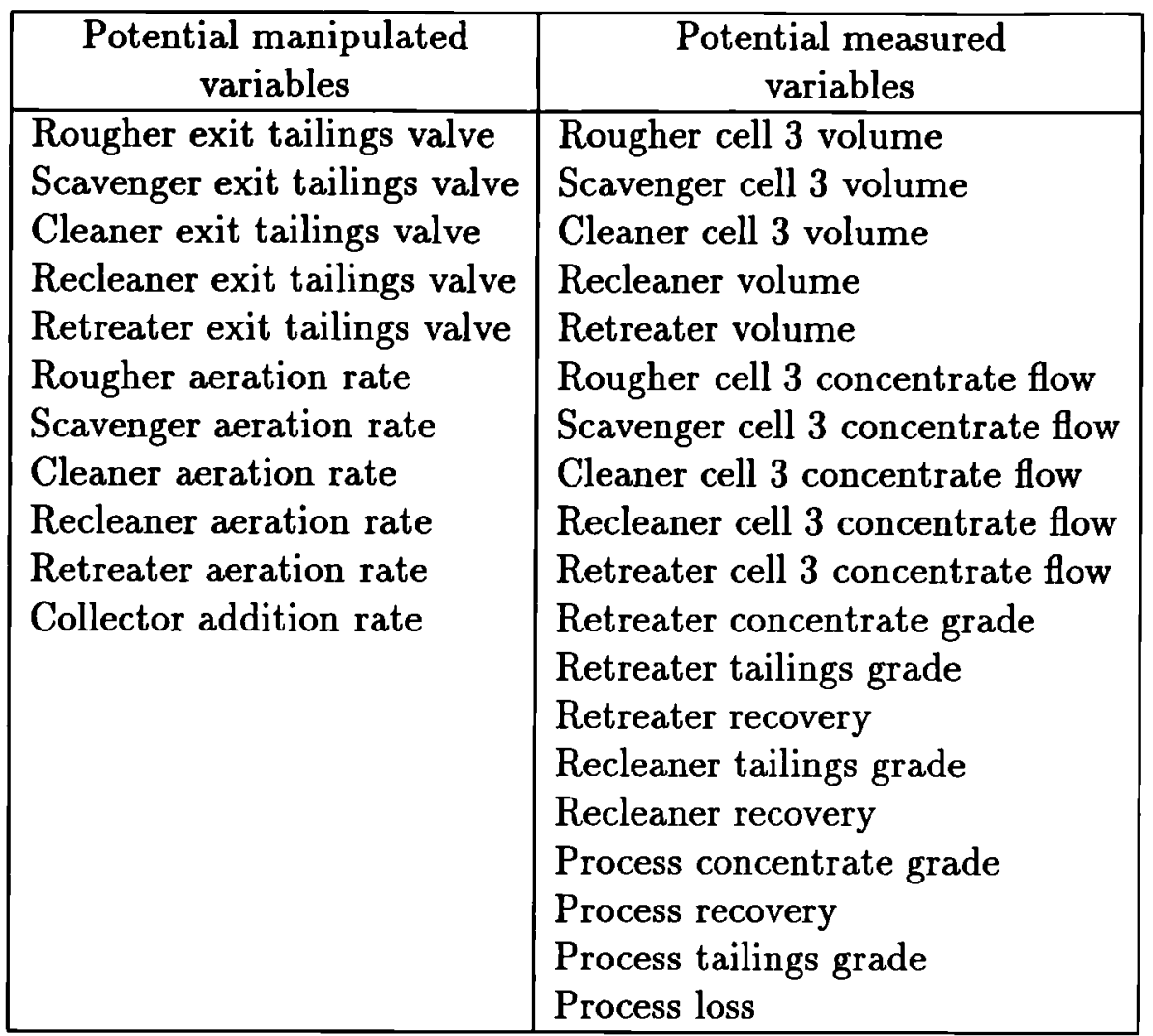

Table 6.17: Potential measured and manipulated variables on the MIM circuit

For the control equipment, two cases were considered to examine the sensitivity of the solution to instrumentation cost. The costing was carried out on the basis of an installed cost with a factor allowing for maintenance of the form: $C_{I}^{\prime}=C_{I}(1+M F)$ where $C_{I}^{\prime}$ is the total instrumentation cost, $C_{I}$ is the installed cost and $M F$ is the maintenance factor. The two cases and their results are now discussed in detail.

\subsubsection{Case 1 - Optimistic sensor/actuator costs}

The first case considered optimistic instrumentation cost estimates, summarised in table 6.18. A maintenance factor, $M F$, of 1 was used for all case 1 instrumentation costs.

The five best control structures and their economic giveaways (relative to the steady state optimum) are summarised in table 6.19 , with minimum condition number plots in figure 6.4. The condition numbers are plotted over a frequency 


\begin{tabular}{|l|c|}
\hline \multicolumn{1}{|c|}{ Variable } & $\begin{array}{c}\text { Installed Cost } \\
(£)\end{array}$ \\
\hline Slurry valve & 2,000 \\
Aeration rate controller & 10,000 \\
Collector addition & 1,000 \\
& \\
Recovery & 15,000 \\
Grade & 15,000 \\
Concentrate flow & 1,000 \\
Volume & 4,000 \\
\hline
\end{tabular}

Table 6.18: Optimistic instrumentation costs for MIM circuit

range corresponding to process noise at 0.5 minutes per cycle to 4 days per cycle corresponding to variation in mining locations. None of the structures contained right half plane zeros.

\begin{tabular}{|ll|ll|}
\hline Structure 1 & $£ 216,450$ & Structure 2 & $£ 225,840$ \\
\hline Cleaner $K_{v}$ & Cleaner volume & Cleaner $K_{v}$ & Cleaner volume \\
Recleaner $K_{v}$ & Product grade & Recleaner $K_{v}$ & Product grade \\
Retreater $K_{v}$ & Retreater volume & Retreater $K_{v}$ & Retreater concentrate flow \\
Rougher $K_{v}$ & Rougher volume & Rougher $K_{v}$ & Rougher volume \\
\hline Structure 3 & $£ 229,260$ & Structure 4 & $£ 238,260$ \\
\hline Cleaner $K_{v}$ & Cleaner volume & Cleaner $K_{v}$ & Cleaner volume \\
Recleaner $K_{v}$ & Product grade & Recleaner $K_{v}$ & Product grade \\
Retreater $K_{v}$ & Retreater volume & Retreater $K_{v}$ & Retreater concentrate flow \\
\hline Structure 5 & $£ 244,020$ & & \\
\hline Cleaner $K_{v}$ & Cleaner volume & & \\
Recleaner $K_{v}$ & Product grade & & \\
Retreater $K_{v}$ & Retreater volume & & \\
Rougher $K_{v}$ & Scavenger volume & & \\
\hline
\end{tabular}

Table 6.19: Optimal control structure for optimistic costs

The first step in the analysis of results is to note the difference in economics between structures 3 and 5, which differ by a single volume measurement and slurry valve. The cost of the additional equipment is $(4000+2000) \times(1+M F)=£ 12,000$. The difference in economics between the structures is: $244020-229260=£ 14,760$, which implies that addition of this control loop has actually degraded the control 


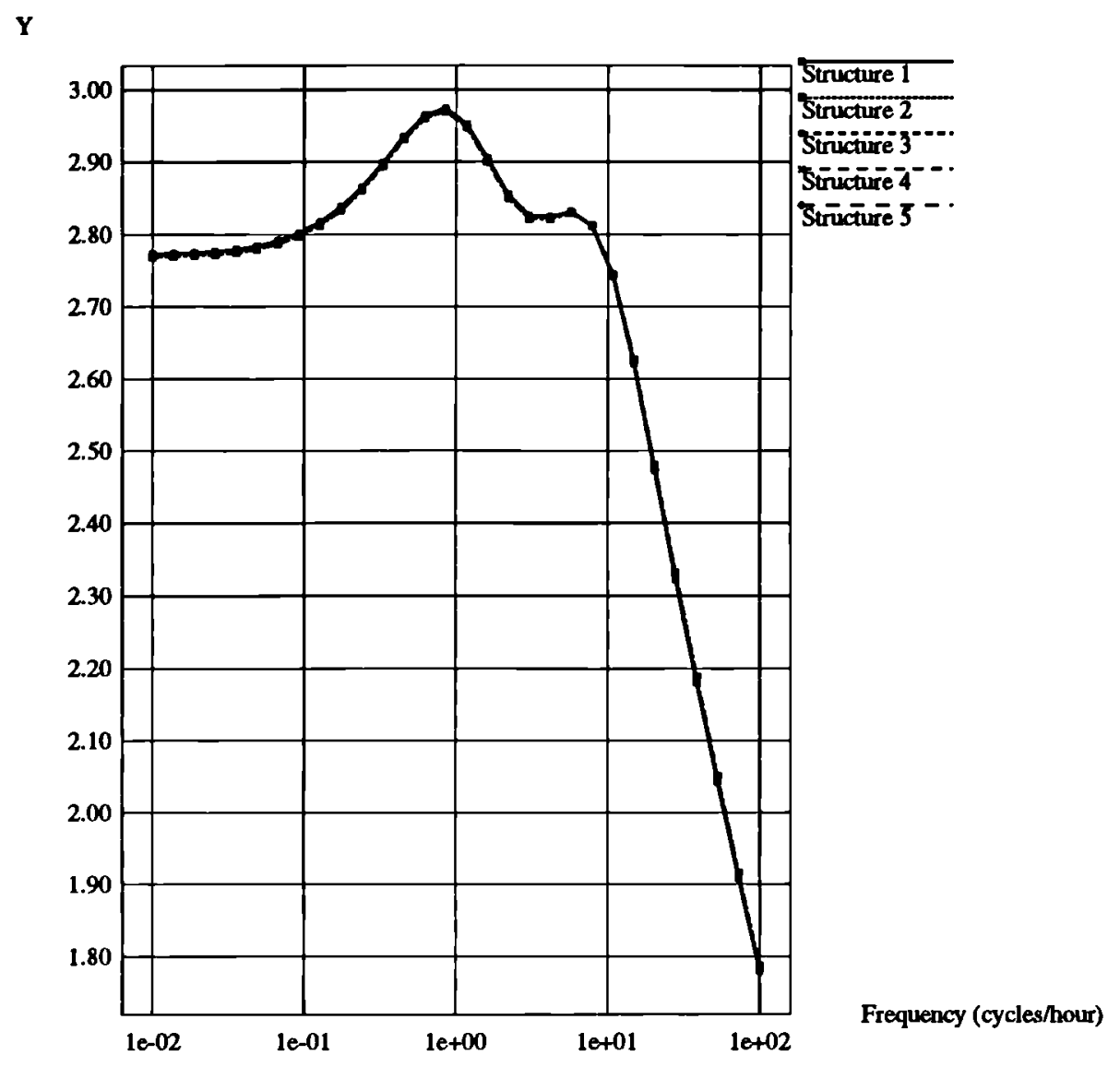

Figure 6.4: $l_{\infty}$ minimum condition number plot for MIM case 1

performance by $£ 2,800$. The reasons for this objective function degradation are explained in the discussion on marginal costs in the following section. From this, it can be concluded that control structure five is an undesirable structure, and will not be examined further. From the remaining four cases, it is clear that there is significant economic benefit to be obtained from controlling the process - potentially, the open loop economic giveaway can be reduced by $70 \%$ to $80 \%$. The next step is to study the controllability indicators to determine the attainability and ease of achieving this control.

Examining the condition number plot (figure 6.4), it can be seen that there is practically no difference in condition numbers between the control structures, indicating that the structures have similar controllability properties. This result may be explained in two stages: First of all consider the first four structures ignoring the rougher valve and the rougher volume in structures one and two, when the only 
difference between the structures is whether the retreater volume or the retreater concentrate flow is controlled. Then it may be noted from the process model that the concentrate flow is related to cell volume by:

$$
C=V a \frac{\sum K_{i} X_{i}}{\sum \frac{X_{1}}{\rho_{i}}}
$$

where $C$ is the concentrate flow, $V$ is the volume, $a$ is the air factor, and $K_{i}, X_{i}, \rho_{i}$ are the rate constants, mass fractions and densities of the components. Linearising at the steady state operating point results in:

$$
\delta C=a \frac{\sum K_{i} X_{i}}{\sum \frac{X_{i}}{\rho_{i}}} \delta V+V a \sum_{j}\left[\frac{K_{j}}{\sum \frac{X_{i}}{\rho_{i}}}-\frac{\sum K_{i} X_{i}}{\rho_{j}\left(\sum \frac{X_{i}}{\rho_{i}}\right)^{2}}\right] \delta X_{j}
$$

where $\delta x$ is the deviation of generic variable $x$, and the remaining variables are at their steady state values. In this case, provided that the $\delta X$ terms either cancel out, or are small relative to the $\delta V$ term, then $\delta C \approx k \delta V$. That is, $C$ varies approximately linearly with $V$, or $C$ is linearly scaled with respect to $V$. As the minimum condition number of a matrix is scale independent, it would be expected that control structures differing only by these two variables would have similar condition numbers. The second effect which causes the similarity of condition numbers is due to the structure of transfer functions containing the rougher valve and volume:

$$
\left[\begin{array}{c}
V_{\text {rougher }} \\
V_{\text {cleaner }} \\
V_{\text {retreater }} \\
\text { Product grade }
\end{array}\right]=\left[\begin{array}{cccc}
g_{11} & 0 & 0 & 0 \\
g_{21} & g_{22} & g_{23} & g_{24} \\
g_{31} & g_{32} & g_{33} & g_{34} \\
g_{41} & g_{42} & g_{43} & g_{44}
\end{array}\right]\left[\begin{array}{c}
K_{v, \text { rougher }} \\
K_{v, \text { cleaner }} \\
K_{v, \text { retreater }} \\
K_{v, \text { recleaner }}
\end{array}\right]
$$

The zero elements occur in the first row as there is no recycle from the process to the rougher. This transfer function has a square block triangular structure, for which the following theorem holds (see appendix $\mathrm{F}$ for proof):

Theorem For a square block triangular matrix $R$ of the form:

$$
R=\left[\begin{array}{ll}
A & 0 \\
Q & B
\end{array}\right]
$$

the $l_{\infty}$ norm minimum condition number $\gamma_{\infty}^{*}$ of $R$ is given by the maximum of the $l_{\infty}$ norm minimum condition numbers of the square submatrices $A$ and $B$, or:

$$
\gamma_{\infty}^{*}(R)=\max \left\{\gamma_{\infty}^{*}(A), \gamma_{\infty}^{*}(B)\right\}
$$


Applying this theorem to the transfer function 6.1, and noting that the minimum condition number of $g_{11}$ is 1 (a SISO system), the minimum condition number of 6.1 is given by the minimum condition number of the remaining three by three subsystem $g_{22}$ to $g_{44}$. Using this result to reconsider the first four control structures, and remembering that condition numbers are expected to be similar whether controlling retreater volume or retreater concentrate flow, it may be stated that the addition of the rougher valve and volume to the control structures will not change the condition numbers. The physical interpretation of this result is that the rougher valve is used to control the rougher volume, and the rougher product acts as a disturbance to the remaining system (as there is no feedback from the process to the rougher).

Given that there is no significant difference in controllability between the first four structures, as they are trying to control essentially the same variables, it is possible to state for this case that control structure one is the best control structure for further development, and as its minimum condition numbers are close to one, very good control should be possible. This is confirmed by considering the location of the manipulated and measured variables on the plant. Each valve and measurement lies on a separate bank, where the banks act as buffers for process disturbances and control interaction. This implies that the control should be largely decoupled, and tight control should be possible with a relatively simple control system.

However, it must also be remembered that a reduced set of measured and manipulated variables were considered for the MILP, due to constraints on solution time. An extension of this analysis is to use the results of the MILP to determine the desired control objectives and check if any of the unused manipulated and measured variables could be used to improve controller performance. Some of the process control objectives may be determined directly from selected measured variables, in this case controlling the product grade on its constraint and controlling the retreater volume at its maximum, while the remaining objectives must be inferred from the effects of the control structures on the constraint slack variations. Considering the first four structures, the control objectives were determined to be:

- Rougher feed cell maximum volume

- Cleaner feed cell maximum volume 
- Retreater maximum volume

- Product minimum grade

From this it was concluded that it was worth considering the rougher feed cell volume and tailings valve and the cleaner feed cell volume and tailings valve in the economic analysis. Various combinations of these and the existing set of measurements and manipulated variables demonstrated that the feed cell valves could not be used for control, as they would saturate, but that measuring the feed cell volumes could improve process performance marginally. In fact, one further control structure with economics better than structure one was located through this method: a giveaway of $£ 215,570$ using the retreater, cleaner final cell and recleaner valves to control the product grade, cleaner feed cell volume and retreater volume. This indicates careful use of the MILP with a subset of the available control structures may yield enough information to improve the control structure. However, this approach should be used cautiously, and only be used if solution time is of critical importance, as the optimal control structures may still be missed.

It should be noted in conventional control system design, the engineer will choose a set of control objectives and then attempt to determine an appropriate control structure for the objectives (Nishida et al., 1981). However, it is recognised that the problem of control objective selection is non-trivial, and using this approach there is no guarantee that the selected control objectives will necessarily be the optimal control objectives. On the other hand, the MILP approach automatically determines the control objectives while selecting the optimal control structure. That is, the MILP approach reverses the control objective, control structure problem by implicitly determining the control objectives which maximise profit. Therefore the MILP approach has some value in simply determining desirable control objectives for design purposes.

\subsubsection{Case 2 - Pessimistic sensor/actuator costs}

The second case, summarised in table 6.20, considers more realistic plant costs, allowing for the high replacement rates of equipment in contact with slurrys and the high maintenance overheads associated with equipment such as X-ray fluorescence composition measurement and magnetic flow meters and densimeters. The 
slurry valves are given a high maintenance factor as they are manipulating abrasive flows. The low maintenance factor on the grade and recovery measurement does not imply that the maintenance factor is low, but that a smaller maintenance factor is needed for an item with a high installed capital cost. The reverse argument holds for the magnetic flow and level meters, which have a low installed cost but require high maintenance for accurate calibration and performance, and hence have a high maintenance factor. The aeration control and collector control have low maintenance factors as they are relatively reliable and do not contact the abrasive streams. The aim of this case is to determine if the choice of instrumentation costs can have a significant impact on the choice of the control structure.

\begin{tabular}{|l|c|c|}
\hline \multicolumn{1}{|c|}{ Variable } & $\begin{array}{c}\text { Installed Cost } \\
(£)\end{array}$ & $\begin{array}{c}\text { Maintenance } \\
\text { factor }\end{array}$ \\
\hline Slurry valve & 10,000 & 3 \\
Aeration rate controller & 10,000 & 1 \\
Collector addition & 2,000 & 1 \\
& & \\
Recovery & 30,000 & 1.5 \\
Grade & 30,000 & 1.5 \\
Concentrate flow & 4,000 & 4 \\
Volume & 8,000 & 4 \\
\hline
\end{tabular}

Table 6.20: Pessimistic instrumentation costs for MIM circuit

The five best control structures are given in table 6.21. The first four control structures correspond to the first four structures for the optimistic case, but in reverse order, and as a result condition numbers are not plotted for this case. The fifth structure again results in a degradation of control performance over structure 1 , with an increase of $£ 1,250$ in the dynamic giveaway after taking the control costs into account. Therefore the fifth structure was again determined to be a poor control structure and ignored in further analysis.

The result of this case indicates that the cost of the instrumentation is significant in choice of control structure, as the economic order of the structures has been reversed between cases 1 and 2 . This is due to changes in the marginal costs of the instrumentation, where the marginal cost is defined as the instrumentation cost minus the improvement in economic performance from instrumentation. The 


\begin{tabular}{|ll|ll|}
\hline Structure 1 & $£ 441,260$ & Structure 2 & $£ 446,260$ \\
\hline Cleaner $K_{v}$ & Cleaner volume & Cleaner $K_{v}$ & Cleaner volume \\
Recleaner $K_{v}$ & Product grade & Recleaner $K_{v}$ & Product grade \\
Retreater $K_{v}$ & Retreater concentrate flow & Retreater $K_{v}$ & Retreater volume \\
\hline Structure 3 & $£ 496,840$ & Structure 4 & $£ 501,450$ \\
\hline Cleaner $K_{v}$ & Cleaner volume & Cleaner $K_{v}$ & Cleaner volume \\
Recleaner $K_{v}$ & Product grade & Recleaner $K_{v}$ & Product grade \\
Retreater $K_{v}$ & Retreater concentrate flow & Retreater $K_{v}$ & Retreater volume \\
Rougher $K_{v}$ & Rougher volume & Rougher $K_{v}$ & Rougher volume \\
\hline Structure 5 & $£ 502,510$ & & \\
\hline Cleaner $K_{v}$ & Cleaner volume & & \\
Recleaner $K_{v}$ & Product grade & & \\
Retreater $K_{v}$ & Retreater volume & & \\
Retreater air & Retreater concentrate flow & & \\
\hline
\end{tabular}

Table 6.21: Optimal control structure for pessimistic costs

MILP minimises the sum of the marginal costs of the instrumentation. Clearly, the marginal cost is dependent on the instrumentation cost assigned by the engineer, and as the cost of a valve/measurement pair rises for a given control structure, it will reach a point where either its marginal cost is positive, or its marginal cost is greater than that of an alternate valve/measurement pair. In the first case the pair should be removed from the control structure, as it will result in degradation of control performance (as was the case for structure 5). In the second case, the alternate pair should replace the current pair, corresponding to the order reversal seen for the first four structures between the pessimistic and optimistic cost cases. From this it can be concluded that if accurate instrumentation costs are not available, then a sensitivity analysis of the results should be carried out to determine if the marginal costs of the controllers will be significant, and if so, a number of potential control structures should continue to be examined until more accurate data is available. In this case it would be recommended that at least structures 1 and 4 be examined further, and preferably also structures 2 and 3.

As the first four structures are identical to the optimistic cost case, the control objectives will also be the same. Re-examining the variable set for alternative control structures revealed two new structures which outperform structure 1: cleaner final cell valve, recleaner valve, and retreater valve controlling cleaner feed 
cell volume, retreater volume and product grade, yielding a giveaway of $£ 432,170$, and the same valves controlling cleaner feed cell volume, retreater concentrate flow and product grade, yielding a giveaway of $£ 427,570$. The second case corresponds to an improvement of approximately $£ 13,000$. This result again emphasises the possibilities of using the MILP as a method for inferring control objectives.

Finally, in both instrumentation cost cases, the economic analysis indicated that control of the process should decrease the open loop economic giveaway by between $60 \%$ and $80 \%$, or by approximately $3.5 \%$ to $4.5 \%$ of the plant steady state costs. In spite of the fact that this is an optimistic estimate (based on perfect control), this result indicates that there is significant incentive for development of a control system for the plant even if the most pessimistic control costs are used. It is also likely that performance similar to that estimated should be possible due to the low condition numbers of the control structures. A more cautious attitude to control would have been required if the results of the sensitivity analysis had indicated that the pessimistic and optimistic economics were closer to the open loop economics.

\subsubsection{Computational results}

The number of major (MILP) linear programming iterations required to reach the solutions, and the number of major iterations to prove optimality are summarised in tables 6.22 and 6.23. The MILP solved approximately 2200 master linear programs per day on a dedicated Sparc II, requiring approximately five and a half days to prove the optimal solutions for both cases, ten days to prove the five best structures for case 1 , and 14 days to prove the five best structures for case 2 . A structure is proved once the MILP predicts a giveaway worse than the perfect control giveaway of the structure. This may seem an extremely long time for solution, but it must be compared with the time required for explicit enumeration of the problem. Using the currently implemented perfect control routine to examine each potential control structure (i.e. 54,000,000 perfect control evaluations), it is estimated that it would require 3 to 6 years to complete, depending on the number of infeasible structures. However the current perfect control routine is fairly inefficient, and it is estimated by appropriate variable elimination, the time for explicit enumeration can be reduced to between 70 to 100 days of Sparc II time. From this it can be concluded that the current MILP implementation is an order of magnitude faster 
than explicit enumeration at locating the optimum solution, and about five times faster at locating a useful number of control structures for further design analysis.

\begin{tabular}{|c|c|c|}
\hline Structure & $\begin{array}{c}\text { Iterations } \\
\text { to solution }\end{array}$ & $\begin{array}{c}\text { Iterations to } \\
\text { prove solution }\end{array}$ \\
\hline 1 & 315 & 12051 \\
2 & 189 & 14756 \\
3 & 448 & 15784 \\
4 & 229 & 19251 \\
5 & 605 & 21614 \\
\hline
\end{tabular}

Table 6.22: Major iterations to solution and prove optimality: case 1

\begin{tabular}{|c|c|c|}
\hline Structure & $\begin{array}{c}\text { Iterations } \\
\text { to solution }\end{array}$ & $\begin{array}{c}\text { Iterations to } \\
\text { prove solution }\end{array}$ \\
\hline 1 & 295 & 12400 \\
2 & 1103 & 13372 \\
3 & 1698 & 28442 \\
4 & 3324 & 30153 \\
5 & 1832 & 30557 \\
\hline
\end{tabular}

Table 6.23: Major iterations to solution and prove optimality: case 2

The current MILP formulation is relatively inefficient, solving only 2200 linear programs per day. By improving the LP description, it should be possible to achieve significant improvements in speed. The second area for improvement is the number of master linear programs required to prove solutions: in this problem, to prove a solution required from 10 to 90 times the number of master LP evaluations required to locate the solution. This appears inefficient, suggesting that it should be possible to improve the termination criteria. It should also be possible to improve the MILP formulation to reduce the integrality gap of the problem. Some of these aspects will be discussed briefly as pointers to future work.

1. The current master problem is extremely large, as it carries redundant constraints and coefficients for a large number of integer cuts. Performance may be improved by only adding integer cut constraints as integer solutions are found (avoiding the need for large numbers of unused cut constraints in the 
problem), and by application of presolution procedures to eliminate redundant constraints and variables. Both of these will act to improve the speed of evaluation of the master linear programs by reducing the number of constraints in the problem.

2. The current termination criterion states that a solution is found once the MILP master predicts an objective function worse than the perfect control objective function of the solution. There are two possibilities to improve the termination criterion. One is to take the approach taken in general MILP problems, where it is only required to achieve the "optimum" solution within a specified margin. In this case the termination criterion is modified such that a solution is declared optimal if the MILP predicts a value worse than the perfect control objective function multiplied by the margin. The second possibility is that a rigorous examination of the MILP formulation may lead to an improved termination criterion. To achieve this improvement would require proof that the current termination criterion is conservative and development of a new bound for MILP termination. This approach would address the problem of excessive numbers of master problems to prove a solution.

3. Re-examination of the MILP formulation may reveal further constraints or improvements to the existing constraints to reduce the integrality gap, and therefore improve the branch and bound performance.

4. In the current implementation, the branch and bound procedure can branch repeatedly on a constant objective function, due to degeneracy in the LP. Performance would be improved by developing a branching criterion which guarantees a worsening in the objective function at every iteration (although there is no guarantee that such a criterion exists), resulting in progress towards the solution at every iteration, rather than remaining static.

5. Finally, the case study results have demonstrated that the MILP performance is highly dependent on the bounds selected for the design and manipulated variables (both steady state and dynamic). In two small test cases, it was demonstrated that relaxing the dynamic manipulated variable bounds by a factor of two resulted in a problem which required more than twice as many 
master linear programs to solve, but did not change the optimum. From this it can be concluded that these bounds should be chosen to reflect the range of the linearisation, and the desirable rangeability of the manipulated variables.

\subsection{Summary}

These case studies have demonstrated the MILP formulation to be an effective method for the selection of process control structures. They also demonstrated that the instrumentation costs for the process are significant in the choice of control structure, as it is the marginal cost (cost of implementing control - improvement in economics from control) that determines the control system economics. Finally it was concluded that, while the MILP method is more effective than explicit enumeration, further improvements should be possible from a more efficient implementation and by refining the problem formulation.

Considering both case studies, it can be seen that a systematic method for assessing control structures has been developed. It may be summarised as:

1. Develop a nonlinear dynamic model of the process

2. Pose and solve a steady state optimum design problem

3. Identify potential manipulated and measured variables, and linearise the process at the steady state optimum using the manipulated variables as inputs and the measured variables and constraint slacks as outputs

4. Identify the most significant disturbance frequency and magnitudes, and cost the sensors/actuators

5. Solve the MILP for a number of control structures

6. Carry out a controllability analysis of these structures, and use in conjunction with the economics to choose the best control structure

7. Carry out a sensitivity analysis as appropriate

8. If an incomplete measurement and manipulated variable set was employed in the MILP, infer the control objectives, and attempt to determine improved control structures. 
The last point has not been discussed in great depth, but in both case studies, it was possible to infer the control objectives from the chosen control structures and examination of the process constraints. This procedure is the reverse of that discussed by Nishida et al (1981), where control system synthesis is defined as starting with selection of the control objectives, and then determining appropriate manipulated and measured variables. This emphasises the point that it is preferable to determine the control objectives implicitly (as is done in the MILP), rather than explicitly, as an explicit set of control objectives are not guaranteed to be the optimal control objectives.

In summary, the MILP method has been demonstrated to be an effective method for selecting control structure, although there is still scope for improvement in performance, and it can be used in a systematic manner to gain information on the process dynamics. 


\section{Chapter 7}

\section{Nonlinear control structure selection}

The two previous chapters considered application of linear analysis methods to the control structure problem. This chapter considers an exploratory extension of these ideas to nonlinear dynamic systems. It is not intended to supply a comprehensive examination of nonlinear dynamic economics, but rather to determine if existing techniques can be utilised for process control structure selection in a simple manner. The problem was approached by taking the combined design problem description discussed in chapter 2 , and removing elements until a soluble problem was developed (while retaining the vital elements of the nonlinear description). The mathematical description of the combined design problem is repeated here as the starting point for the nonlinear control structure selection problem:

$$
\begin{aligned}
& \min _{d, k, X} J\left(x_{0}, z_{0}, u_{0}, d, p_{0}, k, X\right) \\
& f(\dot{x}, x, z, u, d, p)=0 \\
& g(x, z, u, d, p)=0 \\
& h(\dot{x}, x, z, u, d, p) \leq 0 \\
& p=p_{0}+\tilde{p}(t) \\
& u=c(\dot{x}, x, z, u, d, p, k, X) \\
& u^{\text {low }} \leq u \leq u^{\text {high }} \\
& \dot{x}(t=0)=\dot{x}_{0}=0 \\
& D\left(x_{0}, z_{0}, u_{0}, d, p_{0}\right) \leq 0 \\
& d^{\text {low }} \leq d \leq d^{\text {high }} \\
& k^{\text {low }} \leq k \leq k^{\text {high }} \\
& \forall \tilde{p}(t) \in P(t), \forall t \geq 0
\end{aligned}
$$

where $x$ is the vector of state variables (including any controller states), $z$ is the vector of algebraic variables, $p$ is the process disturbance vector made up from the vector of deviations $\tilde{p}$ and the nominal disturbance value $p_{0}, d$ is the vector of design parameters, $u$ is the vector of potential manipulated variables. A subscript 0 refers to the nominal (steady state) values of the variables at time $t=0$. The steady state objective function $J$ is to be optimised subject to steady state constraints $D$ and 
operating constraints $h$ (dynamic path constraints), where the process behaviour is described by the system of state equations, $f$, and algebraic equations, $g$. The dynamic constraints $h$ must be satisfied at all times in the face of disturbance $p$. The steady state design constraints, $D$, must be satisfied at the nominal operating point, and typically consist of design requirements and bounds on equipment sizes and throughputs (these constraints are often linear). There will generally be few design and operating constraints relative to the DAE system size. The controller superstructure is described by the vector of integer variables, $X$, the vector function, $c$, and the vector of controller parameters, $k$. Equations (7.1) must be satisfied for all possible disturbance deviations in the set $P(t)$ at all times $t \geq 0$. The design engineer may vary the design parameters and controller parameters, $d$ and $k$, both of which are subject to simple bounds, along with the integer variables to optimise the process objective function.

As has been discussed previously (chapter 2), this type of problem is extremely difficult to solve. Therefore it would be desirable to consider a simplified problem for initial study. To this end, a number of simplifying assumptions were made:

1. Use of a single dynamic disturbance $\tilde{p}(t)$, from the set of $P(t)$. This assumption is made to avoid the need for including worst case analysis, which results in a mixed integer worst case design problem (the optimisation of equation set 7.1 corresponds to locating a design that satisfies these constraints for all $\tilde{p} \in P(t))$. As this thesis addresses selection of control structures rather than solving large optimisation problems, this is regarded as an acceptable assumption for initial examination of nonlinear dynamic analysis. The issue of worst case disturbances will be returned to if the analysis is successful for the single disturbance case.

2. Integration over a finite time horizon. That is, equations 7.1 must be satisfied from time $t=0$ to some final time $t=t_{f}$. The chosen finite time horizon should be of sufficient length for the process to reach a new steady state or to achieve stable cyclic operation. This assumption is made to allow solution of the problem in a finite time.

3. For linear problems, the perfect control case was considered, as there were 
several applicable linear analysis tools. For the nonlinear problem, the closest approximation to perfect control will be obtained from some form of optimal control problem, where appropriate optimal control profiles must be calculated. Optimal control profiles are either continuous (rare), or more often a discretised set of control actions of varying magnitudes and durations (Biegler, 1990). In the discrete case, both the magnitudes and durations of the control intervals are optimised for each manipulated variable. Practically, it is difficult to decide the appropriate number of control intervals required, as a step disturbance may require only two intervals, whereas a more complex disturbance may require 20 or more intervals. Therefore it was decided to consider the selection and optimisation of a set of simple parameterised continuous profiles - the optimisation of proportional-integral (PI) multiloop controllers. From the process engineering point of view, it is useful to consider PI controllers, as they are the most commonly used industrial controllers (Gavigan, 1991). The use of PI controllers for control structure selection has two significant drawbacks, discussed below:

- Any given control structure is represented several times - for example, any three measured and manipulated variables result in 9 possible 3 by 3 multiloop structures, and the process economics will usually vary with the multiloop structure chosen.

- The selection of a specific optimum multiloop PI controller does not generate a limiting control performance in the manner of the linear perfect control analysis. That is, for a given optimal multiloop PI structure, it may be possible to achieve significantly better control using a more complex controller on the same structure.

The assumption of PI control implies that the problem addressed will be the selection of the optimal multiloop structure instead of selection of the general optimal control structure. The decision to consider initially only PI controllers instead of a more complex approach is justified as follows: (i) it is not known whether there will be any significant benefits to be obtained from nonlinear analysis, and therefore it would be desirable to begin with a simple problem representation to assess the benefits of such an analysis (PI controllers are 
simply parameterised and implemented) and (ii) it is not obvious how the general nonlinear problem should be posed and therefore solving a simpler formulation should give guidance on how to tackle the general problem. In summary, the multiloop problem is considered as it is simple to implement, and should yield valuable information for the formulation and solution of the general nonlinear problem.

Given these assumptions, the problem becomes the selection of the optimal multiloop PI control structure for a process affected by a single disturbance vector over a finite time horizon. The next step is to discuss the representation of the PI controllers.

\subsection{Formulation of PI controllers}

Consider a standard representation of a single PI controller (Stephanopoulos, 1984), with a binary variable $X$ describing whether the controller is active $(X=1)$, or inactive $(X=0)$ :

$$
\begin{aligned}
e & =s-m \\
\Delta u & =K\left(e+\frac{1}{\tau} \int e d t\right) \\
u & =\mu+\Delta u \\
K^{\text {low }} X \leq K & \leq K^{\text {high }} X \\
\tau^{\text {low }} \leq \tau & \leq \tau^{\text {high }} \\
s^{\text {low }} \leq s & \leq s^{\text {high }}
\end{aligned}
$$

or, writing equation 7.2 in DAE form:

$$
\begin{aligned}
\dot{I} & =\frac{e}{\tau} \\
\Delta u & =K(e+I)
\end{aligned}
$$

where $m$ is the measured variable, $e$ is the error, $s$ is the controller set point, $\Delta u$ is the control action, $K$ is the controller gain, $\tau$ is the integral time and $\mu$ is the actuator offset. $s, K, \tau$ and $\mu$ are all optimisation parameters. Upper and lower bounds on the controller parameters are superscripted high and low. The magnitudes (and sign) of the controller gain bounds are based on a span about the inverse of the steady state process gain between the measurement and the manipulated variable 
at the nominal optimum operating point. Similarly, the bounds on the integral time are based on the process time constants. If the controller is inactive $(X=0)$, then $K$ is set to zero, resulting in no control action (the desired result for an inactive controller). The initial condition for the controller is defined to be steady state (by the problem description). This leads to alternate initial conditions for the controller, depending on whether it is active or inactive. If the controller is active $(X=1)$, then the initial condition is:

$$
e(t=0)=0
$$

and otherwise

$$
I(t=0)=0
$$

The reason two initial conditions are required is as follows:

- If the controller is active, then choosing $\Delta u=0$ as the initial condition leads to $u_{0}=\mu$. Solving the plant equation system yields $m_{0}$, that in general will not equal the set point $s$, implying a nonzero error, $e_{0}$, and thus a nonzero value of $\dot{I}_{0}$, violating the steady state initial condition requirement. To satisfy the steady state initial condition, the error at time $t=0$ must be zero, as $\dot{I}_{0}=0=\frac{e_{0}}{\tau}$. The entire system is then solved, determining $I_{0}$ such that $u_{0}$ results in $m_{0}=s$.

- If the controller is inactive, then the error cannot be set to zero, as this will result in a badly specified system as the value of $I_{0}$ is undetermined and it may not be possible to obtain $m_{0}=s$ as the value of $u_{0}$ is fixed to $\mu$ ( $u_{0}$ cannot be varied to obtain the desired $m_{0}$ ). In this case specifying an arbitrary $I_{0}$ gives a valid initial condition.

An alternative representation of the PI controller is:

$$
\begin{aligned}
& e=s-m \\
& \dot{I}=\frac{e}{\tau} \\
& \Delta u=K(e+I) \\
& u=\mu+\Delta u \\
& \dot{s}=0 \\
& K^{\text {low }} X \leq K \leq K^{\text {high }} X \\
& \tau^{\text {low }} \leq \tau \leq \tau^{\text {high }}
\end{aligned}
$$


where $s$ is no longer an optimisation parameter, but is determined by the system initialisation (it remains constant during integration as its gradient is zero). In this case the following initial conditions hold regardless of the value of $X$.

$$
\begin{aligned}
& e(t=0)=0 \\
& I(t=0)=0
\end{aligned}
$$

There are significant advantages to be obtained by using the second representation:

- The set of variables initialised does not change with changes in the integer variables.

- One less optimisation parameter per controller, leading to a decreased NLP solution time.

- Most importantly, it guarantees that the plant will always have its nominal operating point in the steady state operating region. This is because $u(t=0)=\mu$ as $\Delta u(t=0)=0$ by the initial conditions, and $\mu$ is typically subject to simple bounds which define the steady state operating region. Therefore, if there are no infeasible points in the steady state operating region, this formulation guarantees that successful initialisation is possible for each function evaluation. In the first formulation, the set point, $s$, is determined by the optimiser, and it may choose set points which lie outside the steady state operating region, or infeasible set points (no initialisation possible), leading to poor optimiser performance.

As a final note on representation of PI controllers, we have found that the performance of gradient based optimisation methods is improved if the PI controller is written in the form:

$$
\begin{aligned}
e & =s-m \\
\dot{I} & =T e \\
\Delta u & =K e+I \\
u & =\mu+\Delta u \\
\dot{s} & =0
\end{aligned}
$$




$$
\begin{aligned}
K^{\text {low }} X \leq K & \leq K^{\text {high }} X \\
T^{\text {low }} X \leq T & \leq T^{\text {high }} X
\end{aligned}
$$

where $T$ is an optimisation parameter, and the integral action of the PI controller is given by $\tau=K / T$. This results in gradients of the integral action which are not as dependent on the value of the controller gain as the previous representation. Given this representation, we may develop controller superstructures.

\subsubsection{Full superstructure formulation}

A simple superstructure may be developed in the following manner: Assume that the plant contains $n_{m}$ potential measured variables and $n_{u}$ potential manipulated variables. Introduce a set of binary integer variables $X_{i j}$ to indicate whether a PI controller is used to control output $j$ with manipulated variable $i$. Nonlinear dynamic analysis is expected to be computationally expensive, and therefore it would be desirable to eliminate as many poor structures as possible based on structural analysis and engineering judgement. This could be carried out using procedures such as those developed by Morari and Stephanopoulos (1980a) (the previous MILP analysis would not be recommended for highly nonlinear problems). This preanalysis could include eliminating pairings with no structural connection (zero transfer elements), and pairings with poor control performance (e.g. low gains, large time delays and time constants). This should result in the number of controllers included in the structure being less than $n_{u} \times n_{m}$, although probably still of the same order. The superstructure of potential controllers may then be represented as a single controller for each of the $X_{i j}$. An example for a three input, three output plant is shown in figure 7.1. A mathematical representation of this superstructure is:

$$
\begin{aligned}
e_{i j}=s_{i j}-m_{j} & \forall(i, j) \mid \exists X_{i j} \\
\dot{I}_{i j}=T_{i j} e_{i j} & \forall(i, j) \mid \exists X_{i j} \\
\Delta u_{i j}=K_{i j} e_{i j}+I_{i j} & \forall(i, j) \mid \exists X_{i j} \\
u_{i}=\mu_{i}+\sum_{j=j \mid \exists X_{i j}} \Delta u_{i j} & \forall i=1,2, \ldots, n_{u} \\
K_{i j}^{\text {low }} X_{i j} \leq K_{i j} \leq K_{i j}^{\text {high }} X_{i j} & \forall(i, j) \mid \exists X_{i j}
\end{aligned}
$$




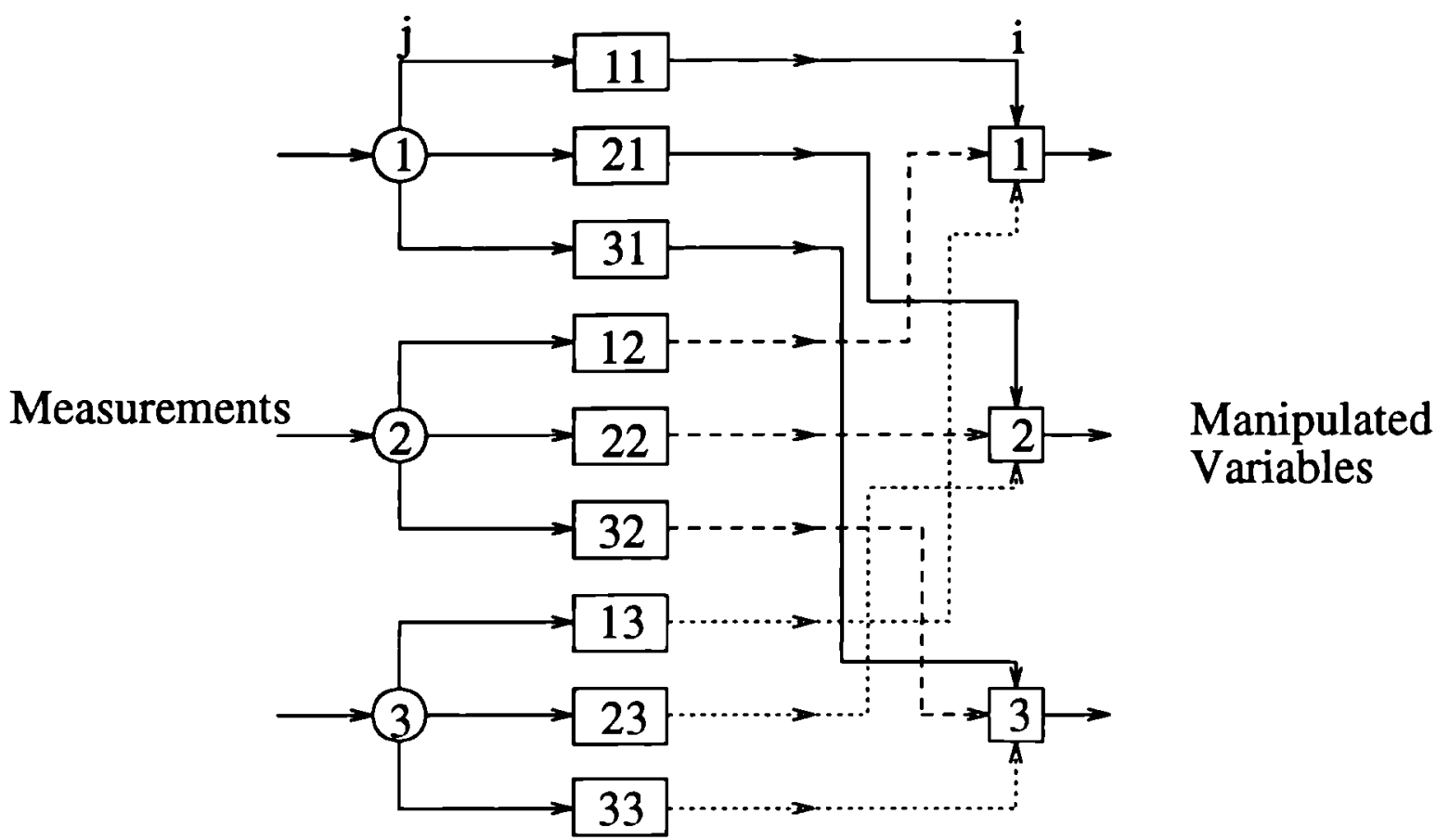

Controllers

Figure 7.1: Full PI controller superstructure

$$
\begin{aligned}
T_{i j}^{\text {low }} X_{i j} \leq T_{i j} \leq T_{i j}^{\text {high }} X_{i j} & \forall(i, j) \mid \exists X_{i j} \\
\sum_{i=i \mid \exists X_{i j}} X_{i j} \leq 1 & \forall j=1,2, \ldots, n_{m} \\
\sum_{j=j \mid \exists X_{i j}} X_{i j} \leq 1 & \forall i=1,2, \ldots, n_{u}
\end{aligned}
$$

where $e, \Delta u, I, T, K, \mu$ and $m$ are row vectors. The notation $\exists X_{i j}$ means for those variables $X_{i j}$ which exist. The integer constraints $7.3,7.4$ require that a manipulated variable be used by at most one controller, and that a measurement be supplied to at most one controller. The integer variables only appear linearly and do not appear in the dynamic equations, which is a prerequisite for some mixed integer nonlinear programming algorithms.

\subsubsection{Shifted superstructure formulation}

An alternative approach to the problem formulation is to employ only as many controllers as either manipulated or measured variables, and then to write an integer superstructure of the possible linkages between controllers and outputs or 
inputs as appropriate. This corresponds to moving the superstructure from around the controllers to between the process and its outputs (or inputs). See figure 7.2 for a diagrammatic representation. To represent this superstructure mathematically, the integer variables must be used in a nonlinear fashion. However, as has been noted, it is desirable to have the integer variables appear linearly. Therefore for notational convenience, a vector of pseudo-integer variables $\mathcal{X}$ is introduced (Kocis and Grossmann, 1988). These variables are then set to the same values as the integer variables (i.e. $\mathcal{X}=X$ ), which remain linear in the problem description.

Considering the case of the superstructure between the controllers and the process, the following description results:

$$
\begin{aligned}
e_{j}=s_{j}-m_{j} & \forall j=1,2, \ldots, n_{m} \\
\dot{I}_{j}=T_{j} e_{j} & \forall j=1,2, \ldots, n_{m} \\
\Delta u_{j}=K_{j} e_{j}+I_{j} & \forall j=1,2, \ldots, n_{m} \\
u_{i}=\mu_{i}+\sum_{j=j \mid \exists X_{i j}} \Delta u_{j} \mathcal{X}_{i j} & \forall i=1,2, \ldots, n_{u} \\
\sum_{i=i \mid \exists X_{i j}} K_{i j}^{\text {low }} X_{i j} \leq K_{j} \leq \sum_{i=i \mid \exists X_{i j}} K_{i j}^{\text {high }} X_{i j} & \forall j \mid \exists X_{i j} \\
\sum_{i=i \mid \exists X_{i j}} T_{i j}^{\text {low }} X_{i j} \leq T_{j} \leq \sum_{i=i \mid \exists X_{i j}} T_{i j}^{\text {high }} X_{i j} & \forall j \mid \exists X_{i j} \\
\sum_{i=i \mid \exists X_{i j}} X_{i j} \leq 1 & \forall j=1,2, \ldots, n_{m} \\
\sum_{j=j \mid \exists X_{i j}} X_{i j} \leq 1 & \forall i=1,2, \ldots, n_{u}
\end{aligned}
$$

A similar description can be written for the case of the superstructure lying between the process and the controllers.

For the application of certain mixed integer programming algorithms, it is undesirable to include nonlinear integer variables in the problem description (Kocis and Grossmann, 1988), as they may introduce nonconvexity into the problem. However, for this problem, there are significant benefits in the reduction of number of variables and optimisation parameters. For any system, the number of continuous variables and parameters is reduced significantly, from 7 variables and parameters $(e, I, s, K, T, \Delta u, \mu)$ per potential controller to 7 variables and parameters per output (or input), plus one pseudo-integer parameter $(\mathcal{X})$ per possible controller. For 


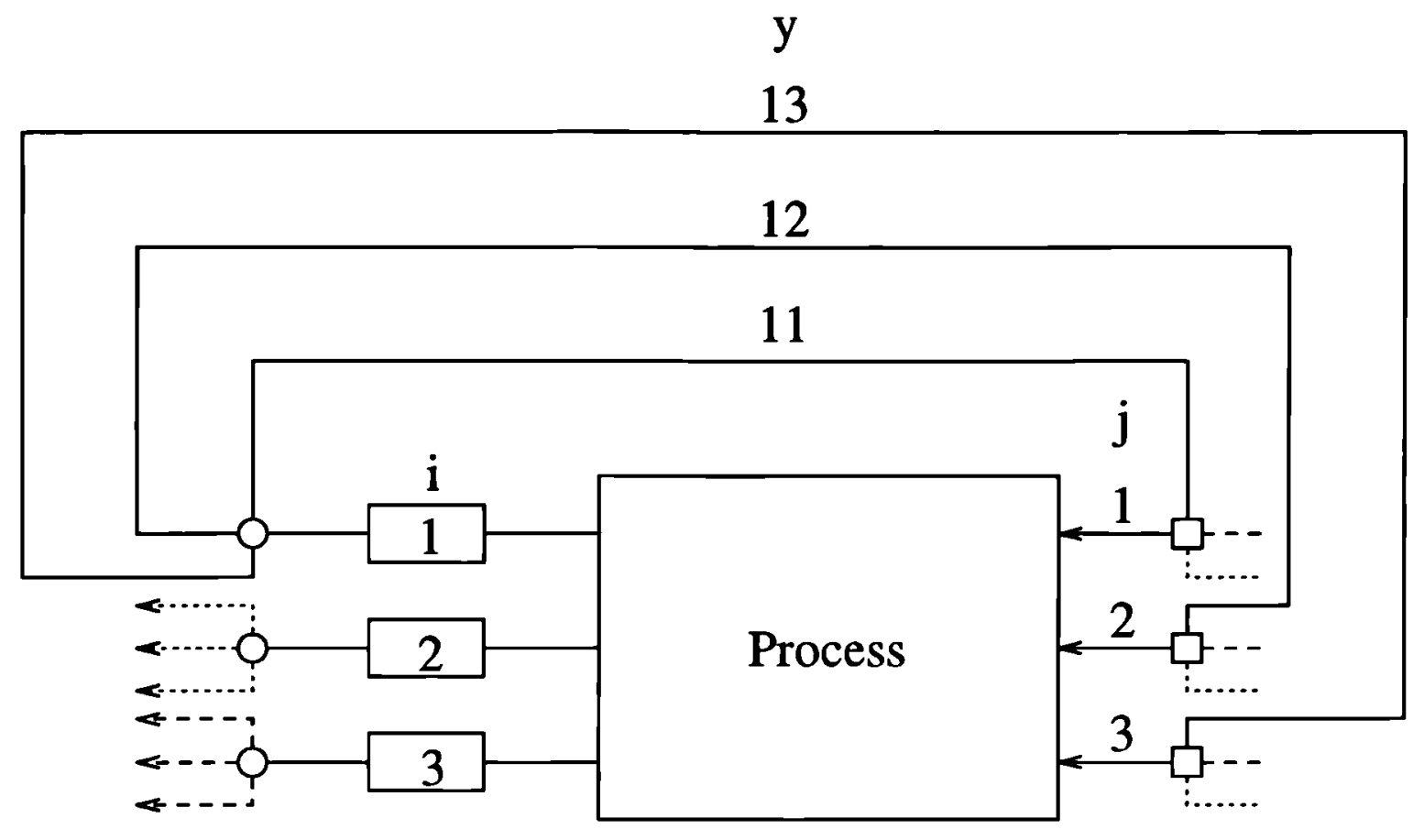

Controllers

Figure 7.2: Shifted controller superstructure

a full matrix $3 \times 3$ system, the full formulation requires 63 variables, compared to 24 for the alternative formulation. The reduction in problem size will increase the speed of function evaluations and reduce the number of optimisation degrees of freedom. Because of the advantages in size and speed to be gained from the shifted superstructure, it will be employed initially and the full superstructure will only be considered if performance is inadequate.

\subsection{Multiloop structure selection problem summary}

Gathering together assumptions and developments of the previous sections, the problem may be written as:

$$
\min _{d, K, T, \mu, X} J\left(x_{0}, z_{0}, u_{0}, d, p_{0}\right)+\epsilon^{T} X
$$




$$
\begin{aligned}
& f(\dot{x}, x, z, u, d, p)=0 \\
& g(x, z, u, d, p)=0 \\
& h(\dot{x}, x, z, u, d, p) \leq 0 \\
& p=p_{0}+\tilde{p}(t) \\
& e_{j}=s_{j}-m_{j} \quad \forall j=1,2, \ldots, n_{m} \\
& \dot{I}_{j}=T_{j} e_{j} \quad \forall j=1,2, \ldots, n_{m} \\
& \Delta u_{j}=K_{j} e_{j}+I_{j} \quad \forall j=1,2, \ldots, n_{m}
\end{aligned}
$$

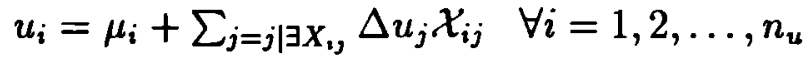

$$
\begin{aligned}
& u^{\text {low }} \leq u \leq u^{\text {high }} \\
& \dot{s}=0 \\
& \dot{x}(t=0)=\dot{x}_{0}=0 \\
& \dot{I}(t=0)=0 \\
& e(t=0)=0 \\
& D\left(x_{0}, z_{0}, u_{0}, d, p_{0}\right) \leq 0 \\
& d^{\text {low }} \leq d \leq d^{\text {high }} \\
& \sum_{i=i \mid \exists X_{1},} K_{i j}^{\text {low }} X_{i j} \leq K_{j} \leq \sum_{i=i \mid \exists X_{1 j}} K_{i j}^{\text {high }} X_{i j} \quad \forall j \mid \exists X_{i j} \\
& \sum_{i=i \mid \exists X_{i},} T_{i j}^{\text {low }} X_{i j} \leq T_{j} \leq \sum_{i=i \mid \exists X_{i}} T_{i j}^{\text {high }} X_{i j} \quad \forall j \mid \exists X_{i j} \\
& \sum_{i=i \mid \exists X_{i}} X_{i j} \leq 1 \quad \forall j=1,2, \ldots, n_{m} \\
& \sum_{j=j \mid \exists X_{i j}} X_{i j} \leq 1 \quad \forall i=1,2, \ldots, n_{u} \\
& \mathcal{X}=X \\
& m \subseteq\{x \cup z\}
\end{aligned}
$$

As the controller states are now represented explicitly, $x$ consists of only the plant states. $\epsilon$ is the vector of control loop costs. The vector of potential measured variables is a subset of the plant state and algebraic variables. This problem is a mixed integer nonlinear programming problem, where the nonlinear program includes optimisation of a nonlinear dynamic system. Problems of the above form will be addressed as mixed integer differential algebraic optimisation (MIDAOPT). Solution of the above problem will require the use of both dynamic optimisation and mixed integer nonlinear programming (MINLP) techniques to solve. Approaches to both of these problems will be briefly discussed, and an appropriate method for 
solving the problem developed.

\subsection{Dynamic optimisation}

Consider the problem:

$$
\begin{aligned}
\min _{\theta} J\left(x\left(t_{f}\right), z\left(t_{f}\right), \theta\right) & \\
f(\dot{x}, x, z, \theta) & =0 \\
g(x, z, \theta) & =0 \\
h(x, z, \theta) & \leq 0 \\
L(x(\phi), z(\phi), \theta) & =0 \forall \phi \in \Phi \\
M(x(\phi), z(\phi), \theta) & \leq 0 \forall \phi \in \Phi \\
0 \leq t \leq t_{f} & \\
\Phi \subset t &
\end{aligned}
$$

This is a dynamic optimisation problem (Biegler, 1990), where $\theta$ is a discrete or continuous control profile or a parameterised controller to be optimised for some disturbance (built into the process description). $L$ and $M$ are a finite set of constraints that must be satisfied at all times in the discrete set $\Phi$. This notation is adopted for convenience in discussing optimal control and can be expanded to consider the case of different constraints that must be satisfied at different times. The objective function may depend on the variable values at other times than $t_{f}$.

Note that this problem description is similar to the problem posed in the previous section, although the latter considers only parameter optimisation.

There are two broad approaches to solving this type of problem: feasible and infeasible path. The infeasible path approach consists of discretising the dynamic equations to a set of algebraic equations and then posing the problem as a large scale NLP. To ensure that $L$ and $M$ are satisfied, all points in the set $\Phi$ must be used as discretisation points with the $L$ and $M$ constraints added directly to the NLP at these points. This NLP may then be solved by a conventional NLP optimiser. The approach is called infeasible path as intermediate solutions will generally 
be infeasible with respect to the original DAE system. Therefore intermediate results may be meaningless and cannot be used as partial solutions if the method fails to find an optimum. Recent work has been carried out in this area by Biegler and colleagues (Biegler, 1990; Cuthrell and Biegler, 1987; Vasantharajan and Biegler, 1990). Generally for single controllers this method leads to large problems that require specialised NLP solvers. The addition of integer variables will increase the problem size dramatically, and will probably require further development of optimisation routines. Based on this and the fact that these methods are still under development, infeasible path techniques have not been examined for use in this project.

The feasible path approach (Sargent and Sullivan, 1977; Morison, 1984; Jang et al., 1987; Mutjaba and Machietto, 1988) consists of converting the path constraint $h$ into a terminal inequality constraint. The optimal control problem may then be posed as a conventional NLP optimisation problem, where function evaluation implicitly integrates the system $f, g$ to evaluate $L, M$ and the terminal path constraints $N$. The reformulation is typically of the form:

$$
\min _{\theta} J\left(x\left(t_{f}\right), z\left(t_{f}\right), \theta\right)
$$

subject to:

$$
\begin{aligned}
& L(x(\phi), z(\phi), \theta)=0 \forall \phi \in \Phi \\
& M(x(\phi), z(\phi), \theta) \leq 0 \forall \phi \in \Phi \\
& N\left(t_{f}\right) \leq 0 \\
& \text { where } \\
& f(\dot{x}, x, z, \theta)=0 \\
& g(x, z, \theta)=0 \\
& \max (h(x, z, \theta), 0)^{2}=\dot{N} \\
& N(t=0)=0 \\
& 0 \leq t \leq t_{f}
\end{aligned}
$$

Appendix $G$ discusses the formulation of path constraints for improved optimisation performance. The feasible path method "hides" the integration by carrying it out during the evaluation of the $J, L, M, N$ functions and their gradients. The values 
and gradients of $L$ and $M$ are obtained by ensuring the integration stops at each point in $\Phi$ and extracting the information directly. The method is called feasible path as the intermediate solutions are generally feasible with respect to the original DAE system. If the method fails to converge, the user can obtain some information and (suboptimal) solutions from the intermediate steps. As this approach can be employed directly using existing numerical integration and small scale optimisation packages, the feasible path method will be used for solution of the dynamic optimisation problem.

Given this description, the MIDAOPT problem may be converted to a mixed integer nonlinear programming (MINLP) problem, where the integration is "hidden" by treating the path constraints $h$ as terminal inequality constraints. That is, the dynamic optimisation becomes a normal nonlinear program, with a set of constraints which require complex evaluation (integration of the system equations). It is important to note that the convexity properties of the terminal constraints have not been quantified, and that the convexity of nonlinear constraints can have a significant effect on the performance of MINLP methods. However, it was decided that rather than attempting an analytical analysis of the curvature of the path constraints, it would be simpler to take an experimental approach, and try to solve the problem using an MINLP method with the capability of handling nonconvex problems.

It should also be noted that any continuous optimisation of the converted MIDAOPT system will require a great deal of time, as each constraint evaluation will require the integration of the system equations. For example, in a small case study consisting of 60 equations, 6 states, 5 path constraints and 5 continuous parameters, optimisation of the system typically required 3 to 5 hours of Sparc I time. Therefore it would also be desirable to select an MINLP method which minimises the number of optimisations. This will be addressed in the next section.

\subsection{MINLP Solution Methods}

The idea of integer programming was introduced in chapter 5 , along with the solution methods of explicit enumeration, Benders decomposition and branch and bound. The problem of mixed integer nonlinear programming has received some 
attention in the literature recently. The most applicable methods for the MIDAOPT problem will be reviewed here, and an appropriate method selected for solution of the problem. There are three requirements attached to the method selected:

1. The method should require as few evaluations of the nonlinear program as possible to minimise computation time.

2. The method must be able to handle nonconvex problems.

3. Many integer programming methods work by solving relaxed integer problems (that is, treat the integer variables as continuous variables for optimisation purposes), and gradually forcing the integrality condition in the search for the optimum. For general MIDAOPT problems, the solution of relaxed problems should be avoided, as partial integer solutions may cause initialisation problems or have no physical meaning. For example, if controller set points were being optimised (as in the case of the first PI representation), then the set of variables initialised changes with the value of the integer variable, and a fractional solution is meaningless. The case study problems (chapter 8) also demonstrated that there can be significant convergence problems, as it proved impossible to solve a relaxed problem for the double effect evaporator, even employing the best integer solution located as a starting point.

Given these requirements, the potential methods will be discussed briefly.

\subsubsection{Explicit enumeration}

The most obvious method of MINLP solution is exhaustive enumeration, that is obtaining the solution by testing every possible combination of binary variables. This approach to the problem requires the evaluation of $2^{n}$ NLP subproblems (i.e. carry out a continuous optimisation for each possible fixed integer vector) where $n$ is the number of binary variables. Exhaustive enumeration guarantees location of the global optimum for nonconvex problems (provided that the solution of each NLP subproblem is the global optimum of that subproblem). However, it can be seen that even for small problems, it will be extremely time consuming to use exhaustive enumeration and therefore it is an inappropriate method for the MIDAOPT problem. 


\subsubsection{Branch and bound}

The branch and bound method (Beale, 1977) is exactly the same as that described for the MILP case (chapter 5), consisting of solving a series of relaxed MINLP problems, and gradually forcing integrality conditions on the integer variables. Intermediate results are used to eliminate poor branches of the search tree. Branch and bound guarantees location of the global optimum (again subject to location of the global optima of the relaxed NLP subproblems), and is generally more efficient than exhaustive enumeration, but has the disadvantage that many relaxed NLP evaluations are required. The number of NLP subproblem evaluations in steady state process synthesis is extremely large (Grossmann, 1990), and has led to the examination of alternative methods for solving MINLPs. As the NLP evaluations for MIDAOPT problems are more computationally expensive than those of steady state synthesis and relaxed problem techniques are undesirable, it is clearly desirable to consider alternative methods that involve fewer NLP subproblem iterations to obtain an acceptable solution speed.

\subsubsection{Feasibility method}

This method is designed for solution of large scale, sparse mixed integer nonlinear programs, and takes advantages of specific features of the optimisation package MINOS (Mawengkang and Murtagh, 1986). It begins by solving a relaxed problem. The integer variables are then partitioned into an inactive set consisting of integer variables with integer values at the relaxed solution, and an active set consisting of the integer variables with fractional values at the solution. The relaxed solution is then rounded heuristically to an integer solution, and a search is performed by altering the variables in the active set in discrete steps. Once a new discrete optimum is determined, the inactive set is re-examined to determine if any of its elements should be added to the active set. This search procedure is then repeated, and the method terminates if no variables are added to the active set.

The method suffers from a number of disadvantages for the MIDAOPT problem: it is intended for sparse problems (the MIDAOPT problems tend to be dense); it solves a relaxed subproblem; optimality is not guaranteed even for convex problems (Viswanathan and Grossmann, 1990); and it is specific to the optimisation 
package MINOS. It is therefore regarded as unsuitable for the MIDAOPT problem.

\subsubsection{Outer Approximation methods}

These methods use a problem decomposition approach, where a master problem approximates the entire feasible region of MINLP, but in a form that is easier to solve for an integer search point. Each integer search point is then solved as an NLP subproblem. Typically the problem is decomposed into an MILP master (relatively easily solved) and a series of NLP subproblems. The strategy consists of successively solving the master and the NLP subproblems, and using the results of the NLP subproblems to augment the master description of the problem. The solution of the augmented master will normally supply a new integer search point and a lower bound on the objective function (although this bound will not generally be valid in the event of a nonconvex problem).

There are two general outer approximation methods: The Generalised Benders Decomposition (GBD) (Geoffrion, 1972), a general MINLP solver; and the Outer Approximation/Equality Relaxation (OA/ER) methods of Grossmann et al (Duran and Grossmann, 1986b; Kocis and Grossmann, 1987; Viswanathan and Grossmann, 1990). The GBD strategy requires many major iterations solving the MILP master and the NLP subproblems, as the master is not a tight approximation of the MINLP, whereas the OA/ER strategy requires fewer major iterations, but at the expense of an increased master problem size (Viswanathan and Grossmann, 1990). For the design problem we are considering, the expense of solving the MILP master problem for either method is slight compared to the cost of solving the NLP subproblem (a relatively small problem with 5 integer variables, integrating about 60 equations and 11 continuous parameters requires 1 to 2 hours to locate an NLP solution on a Sparcstation II, and the MILP master solves in seconds). Therefore it seems most appropriate to select the OA/ER method, as it should require fewer NLP subproblem evaluations. The basis of OA/ER method, and its main variant, the OA/ER Augmented Penalty (OA/ER/AP) method will be further discussed in the following sections. 


\section{Outer Approximation/Equality Relaxation (OA/ER) methods}

The OA/ER algorithm and its main variant, the OA/ ER Augmented Penalty (OA/ER/AP) algorithm have been developed over the past 6 years by Grossmann and colleagues: OA/ER (Duran and Grossmann, 1986a; Duran and Grossmann, 1986b; Kocis and Grossmann, 1987); nonconvex OA/ER (Kocis and Grossmann, 1988); OA/ER/AP (Viswanathan and Grossmann, 1990). Outer approximation refers to the approximation of nonlinear inequality constraints by linear inequality constraints that are consistently more conservative than the original nonlinear constraints, subject to certain convexity conditions on the nonlinear constraints. That is, OA overestimates the feasible region and underestimates the objective function. Equality relaxation is a method for relaxing equality constraints into inequality constraints at NLP subproblem optima. Outer approximation may then be applied to the relaxed constraints. Finally, augmented penalty approaches allow penalised violations of the OA/ER master problem for solution of nonconvex problems (i.e. nonconvex integer solutions that are cut off by the OA/ER algorithm may be examined using an augmented penalty method).

The next question to address is: which of the OA/ER and OA/ER/AP algorithms is most appropriate to solve the design problem posed previously? The two main issues here are nonconvexity handling and size of the MILP master. Addressing the size of the master problem first, it is clear from the problem formulation that the OA/ER/AP strategy will result in a significantly larger master problem than the OA/ER strategy. This is because OA/ER/AP introduces an additional slack variable for every potentially nonconvex constraint at each iteration (to allow the penalised violation of the master region), whereas the number of variables for $\mathrm{OA} / \mathrm{ER}$ remains constant with each iteration. However, as mentioned previously, the time to evaluate the master is small compared to the effort for NLP subproblem evaluation.

The issue of nonconvexities is much more significant, as it is highly likely that most of the terminal path constraints $(N)$ will exhibit significant nonconvexity. As the convexity of these constraints will not be known a priori, it is important that the MINLP method is capable of automatically handling nonconvex problems. For the OA/ER method there are two methods of handling nonconvexity. The first is the two phase strategy of normal OA/ER followed by further relaxation of noncon- 
vex constraints. The method does not guarantee location of the global optimum, but was successful in locating the global optimum in $80 \%$ of the cases examined (Kocis and Grossmann, 1988). This method requires a significant amount of effort to implement. The second method is the use of integer variables to switch nonconvex constraints on and off. This method is simpler to implement, but is less likely to locate a nonconvex global optimum, as some parts of the MINLP feasible region consistently remain infeasible in the master problem. The OA/ER/AP method does not require explicit identification of nonconvexities, as violation of the $\mathrm{OA} / \mathrm{ER}$ master (MILP) region is allowed by the addition of slack variables to the linearisations of the potentially nonconvex constraints. Appropriate penalty weights are applied to these slack variables to encourage the solution to remain within the $\mathrm{OA} / \mathrm{ER}$ master feasible region. This method is an automatic implementation of the local nonconvexity identification in the two phase OA/ER procedure. This algorithm has been found to be computationally efficient and robust for finding the global optimum (Viswanathan and Grossmann, 1990). Based on the arguments of ease of implementation and effectiveness, the OA/ER/AP algorithm will be used as the framework for solving the MIDAOPT problem.

\section{OA/ER/AP algorithm}

This algorithm will be outlined for the following MINLP problem:

$$
\begin{gathered}
J=\min _{d, X} \alpha^{T} X+\mathcal{J}(d) \\
\text { s.t. } g(d)=0 \\
h(d) \leq 0 \\
A d=a \\
B X+C d \leq b \\
d \in\left\{d \mid d \in R^{n}, d^{\text {low }} \leq d \leq d^{\text {high }}\right\} \\
X \in\left\{X \mid X \in\{0,1\}^{m}\right\}
\end{gathered}
$$

Note that the MIDAOPT problem conforms to this description, assuming that the dynamics are "hidden" by reformulation of the path constraints as terminal inequality constraints. 
The OA/ER/AP (DICOPT ++) strategy is given by (Viswanathan and Grossmann, 1990):

1. Set the iteration counter $\Pi$ to zero. Solve the first NLP with the integrality constraints on $X$ relaxed (i.e. $0 \leq X \leq 1$ ) to yield $X^{0}, d^{0}$.

2. Obtain the $\Pi^{\text {th }}$ linear approximations to $\mathcal{J}(d), h(d), g(d)$ at $d^{\Pi}$, and set up the following MILP master.

$$
J_{A}^{\Pi}=\min \alpha^{T} X+\nu+\sum_{\pi} w_{0}^{\pi} s_{0}^{\pi}+\sum_{\pi} \sum_{i} w_{p_{i}}^{\pi} p_{i}^{\pi}+\sum_{\pi} \sum_{i} w_{q_{i}}^{\pi} q_{i}^{\pi}
$$

subject to

$$
\left.\begin{array}{r}
\mathcal{J}\left(d^{\pi}\right)+\nabla \mathcal{J}\left(d^{\pi}\right)^{T}\left(d-d^{\pi}\right)-\nu \leq s_{0}^{\pi} \\
\left.\Gamma^{\pi}\left[g\left(d^{\pi}\right)+\nabla g\left(d^{\pi}\right)^{T}\left(d-d^{\pi}\right)\right] \leq p^{\pi}\right\} \\
h\left(d^{\pi}\right)+\nabla h\left(d^{\pi}\right)^{T}\left(d-d^{\pi}\right) \leq q^{\pi}
\end{array}\right\} \quad \pi=1,2, \ldots, \Pi
$$

$\Gamma$ is the diagonal equality relaxation direction matrix that determines the direction of the relaxation of nonlinear equality constraints into inequality constraints, and is given by:

$$
\Gamma_{i i}^{\pi}=\left\{\begin{array}{c}
-1 \text { if } \lambda_{i}^{\pi}<0 \\
+1 \text { if } \lambda_{i}^{\pi}>0 \\
0 \text { if } \lambda_{i}^{\pi}=0
\end{array} \quad i=1,2, \ldots, r\right.
$$

where the $\lambda$ 's are the Lagrange multipliers of $g(d)=0$. The method locates nonconvex solutions using the slack variables, $s_{0}^{\pi}, p_{i}^{\pi}, q_{i}^{\pi}$, which allow violation of the linearised constraints. Penalty weights $w$ are associated with the slack variables to encourage solutions within the master feasible region. A value of 1000 times the absolute magnitude of the appropriate Lagrange multipliers is suggested as a suitable weight for these problems (Viswanathan and Grossmann, 1990). The $g\left(d^{\pi}\right)$ term will be zero for a feasible subproblem. 
3. Solve the MILP master to yield $X^{\Pi}$ and $J_{A}^{\Pi}$. Solve the NLP subproblem at $X^{\Pi}$.

4. Test for termination using the following criterion: If $J_{1}$ and $J_{2}$ are the objective functions for two sequential feasible NLP subproblems and $J_{2}$ is the most recent, then terminate if $J_{2}>J_{1}$. This criterion does not guarantee location of the global optimum for nonconvex problems, but has shown good performance (Viswanathan and Grossmann, 1990). If the termination criterion is not satisfied, set $\Pi=\Pi+1$ and go to step 2 .

Note in step one a completely relaxed problem is solved. As has been discussed, this is undesirable for the MIDAOPT problem, and therefore an integer solution will be used as a starting point. In this case, it is suggested that several integer starting points are considered to ensure location of the global optimum.

\section{Infeasible NLP subproblems}

In the event of an infeasible NLP subproblem there are two options:

1. Perform an integer cut and continue.

2. Augment the problem to allow a feasible solution. Then relinearise the augmented problem to obtain another set of linearisations for improving the master representation of the MINLP. The augmentation is carried out by reformulating the MINLP as:

$$
\begin{array}{r}
J=\min _{d, X, r} \alpha^{T} X+\mathcal{J}(d)+\rho r \\
\text { s.t. } g(d)=0 \\
h(d) \leq r \\
A d=a \\
B X+C d-b \leq r
\end{array}
$$

Where $\rho$ is a large scalar. Given the effort for NLP evaluations, the first option is used for the MIDAOPT problem. 


\subsection{Implementation}

The OA/ER/AP MINLP algorithm was implemented in Pascal using variants on the branch and bound routines developed by Shah (1992) for solution of the MILP master problems, and employing MINOS (Murtagh and Saunders, 1983) for solution of the LP subproblems. The dynamic optimisation code developed by Walsh (1992) was used for solution of the NLP subproblems. The dynamic optimisation code uses the successive quadratic programming routine SRQPD (Chen, 1988) for solution of the NLP, and the numerical integration package DASOLV (Jarvis and Pantelides, 1992) for the solution of the DAE system. The dynamic optimisation code has been extensively validated on several industrial case studies (Walsh, 1992).

The OA/ER/AP code was validated against the nonconvex test problems EX-3 and MIPB1 from Kocis and Grossmann (1988). The code was tested against the results supplied and against hand calculations, and demonstrated performance consistent with that reported by Kocis and Grossmann. 


\section{Chapter 8}

\section{MIDAOPT case studies}

The Chan et al flotation circuit and the double effect evaporator case studies considered earlier in the thesis were reused as MIDAOPT case studies, allowing rapid development and testing of the approach and comparison of the MIDAOPT results with previous solutions.

\subsection{Chan et al flotation circuits}

This case study is a small problem with few potential control loops, providing a simple test for the MIDAOPT approach. For this study, flowsheets 1 and 8 were examined as these showed the main aspects of the four flowsheets examined previously. The full details of the case study are outlined in chapter 4, and only relevant details are repeated here. The discussion refers to both flowsheets, unless a specific differentiation is made.

The following process variables were identified: the three cell volumes as design variables, the air factor and the feed water as manipulated variables, and the product grade, product recovery and percentage water in the feed as measured variables. There is no structural connection between the air factor and the percentage water in the feed, giving five feasible control loops, listed in table 8.1. A control structure for this plant will consist of one, two or no PI loops, resulting in ten potential multiloop structures.

\begin{tabular}{|ll|}
\hline Manipulated variable & Measured Variable \\
\hline Air factor & Product grade \\
Air factor & Product recovery \\
Feed water & Product grade \\
Feed water & Product recovery \\
Feed water & Percentage water in feed \\
\hline
\end{tabular}

Table 8.1: Possible flotation circuit control loops

The process constraints and acceptable path constraint violations (see ap- 
pendix G) are summarised as:

1. The operating air factor must lie between 0.5 and 1.0 ( 0.001 over 0.01 minutes).

2. The feed water flowrate must lie between 800 and $1022 \mathrm{~kg} / \mathrm{min}(0.1 \mathrm{~kg} / \mathrm{min}$ over 0.01 minutes).

3. The percentage water in the feed must be greater than $46 \%(0.1 \%$ over 0.01 minutes).

4. The product grade must be greater than $60 \%$ ( $0.01 \%$ over 0.01 minutes).

5. The sum of the cell volumes must be less than $54 \mathrm{~m}^{3}$.

The first four are path constraints and the final constraint is a design constraint. For consistency with the previous study of this problem, the controller costs were assumed to be negligible. The process disturbances consisted of in phase sinusoidal variations in the feed valuables and gangue at a frequency of 0.05 cycles per min, with amplitudes of $14.4 \mathrm{~kg} / \mathrm{min}$ and $105.6 \mathrm{~kg} / \mathrm{min}$ respectively. A time horizon of 100 minutes was used, allowing significant initial transients to die away.

The global optimum control structure for both flowsheets was evaluated by explicit enumeration of all of the possible multiloop configurations, assuming that the NLP solver located the global optimum for each structure examined. Flowsheet 1 had two equal optima:- air-recovery, water-water feed percentage and air-grade, water-water feed percentage with a recovery of $93.243 \%$ (steady state $93.261 \%$ ), and flowsheet 8 had a single optimal solution:- air-recovery, water-water feed percentage with a recovery of $94.863 \%$ (steady state $94.987 \%$ ). It can be seen from this result that very effective control of the process can be achieved using simple multiloop controllers, with a loss in recovery of $0.01 \%$ for flowsheet 1 , and $0.1 \%$ for flowsheet 8 , similar magnitudes to those predicted previously by the linear perfect control analysis. However, for both flowsheets, a previously unconsidered control configuration was found to be an optimal solution:- the air-recovery, water-water feed percentage. This result re-emphasises the need for a systematic methodology for the generation and evaluation of process control structures to ensure solutions such as this are not missed. Note that it proved impossible to converge the airgrade, water-recovery pairing, a result consistent with the inability to tune a BLT controller for the same pairing in chapter 4 . 
This study also re-emphasises the need to examine the instrumentation costs and by implication the controller marginal costs (section 6.2.2) to ensure location of the optimal structure. This follows from examining the optimal solutions and noting that the air-recovery and air-grade loops are almost completely detuned. The detuning is shown clearly by comparing the performance of the system with only the water-water loop activated to the optimal solutions:- for flowsheet 1 the difference in recovery is $0.0017 \%$, and for flowsheet 8 the difference is $0.0001 \%$. In both cases the process uncertainty will be of greater significance than the improvement obtained using the air loops. That is, almost all of the economically significant control is carried out by the water-water loop. Therefore, a practical plant controller would employ only the water-water loop. If the instrumentation costs for the control loops had been included then it would be expected that neither the air-recovery nor the air-grade loops would appear in the optimal solutions, as their marginal costs would result in degradation of the objective function. In short, if the problem description does not include all of the economic information, it is likely that a non-optimal structure will be selected.

To test the effectiveness of the MIDAOPT approach at locating the global optimum, both problems were solved starting from all 10 possible multiloop configurations. The shifted superstructure representation of the problem was used, with results summarised in tables 8.2 and 8.3 :

\begin{tabular}{|c|c|c|}
\hline $\begin{array}{l}\text { Initial } \\
\text { Configuration }\end{array}$ & $\begin{array}{l}\text { Solution } \\
\text { Configuration }\end{array}$ & $\begin{array}{c}\text { Major } \\
\text { Iterations }\end{array}$ \\
\hline open loop & air-recovery, water-water & 2 \\
\hline water-water & air-grade, water-water & 2 \\
\hline water-recovery & air-recovery, water-water & 2 \\
\hline water-grade & air-recovery, water-water & 2 \\
\hline air-recovery & air-recovery, water-water & 3 \\
\hline air-recovery, water-water & air-recovery, water-water & 1 \\
\hline air-recovery, water-grade & air-recovery, water-water & 3 \\
\hline air-grade & air-recovery, water-water & 2 \\
\hline air-grade, water-water & air-grade, water-water & 1 \\
\hline air-grade, water-recovery & Failed to converge & \\
\hline
\end{tabular}

Table 8.2: MIDAOPT results for flotation circuit 1

These results demonstrate the effectiveness of the MIDAOPT approach - 


\begin{tabular}{|c|c|c|}
\hline $\begin{array}{l}\text { Initial } \\
\text { Configuration }\end{array}$ & $\begin{array}{l}\text { Solution } \\
\text { Configuration }\end{array}$ & $\begin{array}{c}\text { Major } \\
\text { Iterations }\end{array}$ \\
\hline open loop & air-recovery, water-water & $\overline{2}$ \\
\hline water-water & air-recovery, water-water & 2 \\
\hline water-recovery & air-recovery, water-water & 3 \\
\hline water-grade & air-recovery, water-water & 2 \\
\hline air-recovery & air-recovery, water-water & 3 \\
\hline air-recovery, water-water & air-recovery, water-water & 1 \\
\hline air-recovery, water-grade & air-recovery, water-water & 3 \\
\hline air-grade & air-recovery, water-water & 2 \\
\hline air-grade, wate & air-recovery, water-water & 2 \\
\hline air-grade, & Failed to converge & \\
\hline
\end{tabular}

Table 8.3: MIDAOPT results for flotation circuit 8

in all converged runs, the optimum was located within three major iterations. Note that a minimum of two major iterations are required if the initial configuration is not the optimal solution. These results show that the MIDAOPT approach has potential for use in the control structure selection problem. However, it must be remembered that this problem contains only five integer variables. Before accepting the method as a useful tool, validation should be carried out on a larger problem. To this end, the double effect evaporator will be re-examined in the following section.

MIDAOPT solution time typically required 4 to 8 hours dedicated Sparc I processing, solving between 2 to 4 NLP subproblems, where each subproblem was solved in 40 to 80 function evaluations (integrations). Given the length of time required for evaluation of such a small problem, further thought should be given to improved formulations of the MIDAOPT problem and improved methods for solving the dynamic optimisation subproblem.

\subsection{Double effect evaporator}

This section uses the double effect evaporator from chapter 5 as a larger test problem for the MIDAOPT approach. It was decided to use only a subset of the possible measured and manipulated variables on the plant to maintain problem tractability. This subset of variables was chosen from the variables which appeared in the optimal control structures determined by the MILP method. The feed 
liquor valve, $L V 1$, and the intermediate vapour valve, $V V 2$, were treated as design parameters (only optimising their nominal values). The controller superstructure consists of the four remaining valves $V V 1, V V 3, L V 2$ and $L V 3$ (see figure 6.1) and the following measurements: effect 2 temperature $T$; effect 2 pressure $P$; product composition $X$; product flow $F_{t}$; product solute flow $F_{s}$; product water flow $F_{w}$; effect 1 steam chest pressure $P_{c}$; and effect 1 steam chest temperature $T_{c}$. The superstructure was further limited by assuming that the utility steam valve, $V V 3$, and the steam chest temperature and pressure form a separate subsystem for the purposes of structure selection (i.e. these variables may not form control loops with the remaining variables). This results in two independent subsystems of loops for enumeration of potential multiloop controllers. The steam valve, $V V 1$, may be used to control either $T_{c}$, or $P_{c}$, or may be used open loop, giving 3 potential loops in the first subsystem. Noting that the transfer function between the remaining three valves and six measurements is full (i.e. no zero elements), the number of potential multiloop structures may be calculated as follows. There are $6 \times 5 \times 4=120$ possible $3 \times 3$ structures. If only two valves are active, there are $6 \times 5=30$ possible $2 \times 2$ structures, and 3 combinations of two valves yielding a total of 90 possible $2 \times 2$ structures. Similarly for one active valve, there are 6 possible loops, and there are three combinations of one valve, giving 18 possible single loop structures. Finally, there is the open loop structure. This sums to 229 possible multiloop structures for the second subsystem, and the total number of possible structures for the flowsheet is given by the product of the number of structures in the subsystems, giving $3 \times 229=687$ potential multiloop controllers.

The objective function and instrumentation costs used were the same as for the MILP case. Note that the problem considers optimisation of the operation of prespecified plant items, and therefore includes only operating constraints (i.e. all of the nonlinear constraints are path constraints). These constraints were examined, and all inactive and redundant constraints were removed to speed the integration of the problem, as each operating constraint adds a state when converted from a path constraint to a terminal constraint. The resulting set of constraints and their maximum acceptable violations (appendix $\mathrm{G}$ ) are summarised below.

1. Evaporator holdups of between $3.25 \mathrm{~m}^{3}$ and $4.7 \mathrm{~m}^{3}\left(0.01 \mathrm{~m}^{3}\right.$ over 5 minutes $)$ 
2. A minimum temperature difference of $5^{\circ} \mathrm{C}$ for heat exchange in both effects $\left(0.01^{\circ} \mathrm{C}\right.$ over 5 minutes)

3. A maximum liquor temperature of $95^{\circ} \mathrm{C}$ in the first effect $\left(0.01^{\circ} \mathrm{C}\right.$ over 5 minutes)

4. A minimum solute weight fraction of 0.1 in the product ( 0.001 over 1 minute)

5. A minimum solute flow of $3.5 \mathrm{~kg} / \mathrm{s}(0.01 \mathrm{~kg} / \mathrm{s}$ over 5 minutes $)$

6. A minimum pressure drop across each vapour valve of $5 \mathrm{kPa}(0.01 \mathrm{kPa}$ over 5 minutes)

The disturbance amplitudes and frequency were chosen to be the same as the disturbance for the base case MILP study of the evaporator. The disturbance was taken as in phase sinusoidal variations in: feed pressure (disturbance amplitude of $10 \mathrm{kPa})$; feed temperature $\left(5^{\circ} \mathrm{C}\right)$; feed composition ( 0.0035 weight fraction); utility steam temperature $\left(0.5^{\circ} \mathrm{C}\right)$; and product liquid pressure $(5 \mathrm{kPa})$, all at a frequency of 0.005 cycles per minute. A time horizon of 24 hours was used for the integration, which is sufficient for initial transients to decay.

The final modification consisted of scaling the controller action and parameter bounds descriptions, as this was found to improve NLP convergence. The modifications from the formulation in chapter 8 are:

$$
\begin{aligned}
\dot{I}_{j}=\mathcal{T}_{j} e_{j} & \forall j=1,2, \ldots, n_{m} \\
\Delta u_{j}=\mathcal{K}_{j} e_{j}+I_{j} & \forall j=1,2, \ldots, n_{m} \\
u_{i}=\mu_{i}+\sum_{j=j \mid \exists X_{i j}} \Delta u_{j} \mathcal{X}_{i j} \kappa_{i j} & \forall i=1,2, \ldots, n_{u} \\
\mathcal{K}^{\text {low }} \sum_{i=i \mid \exists X_{i j}} X_{i j} \leq \mathcal{K}_{j} \leq \mathcal{K}^{\text {high }} \sum_{i=i \mid \exists X_{\imath},} X_{i j} & \forall j \mid \exists X_{i j} \\
\mathcal{T}^{\text {low }} \sum_{i=i \mid \exists X_{i j}} X_{i j} \leq \mathcal{T}_{j} \leq \mathcal{T}^{\text {high }} \sum_{i=\imath \mid \exists X_{\imath j}} X_{i j} & \forall j \mid \exists X_{i j}
\end{aligned}
$$

where $\mathcal{K}^{\text {low }}, \mathcal{K}^{\text {high }}, \mathcal{T}^{\text {high }}$ and $\mathcal{T}^{\text {high }}$ are scalar bounds on the scaled vectors of gains and integral actions $\mathcal{K}$ and $\mathcal{T}$ and the $\kappa_{i j}$ are scale factors relating the actual gains and integral actions $K$ and $T$ to their scaled counterparts.

As has been noted, the problem contains over 600 potential control structures. It would require several months to examine all of these structures, and as a 
result explicit enumeration was regarded as impractical for this study. Therefore, it was decided to examine the MIDAOPT performance from 20 sensible starting points, summarised in table 8.4. The problem was solved using the shifted superstructure representation.

\begin{tabular}{|c|l|}
\hline Case & Initial structure \\
\hline 1 & open loop \\
2 & $V V 3-T, L V 2-P$ \\
3 & $V V 3-P, L V 2-T$ \\
4 & $V V 3-T, L V 2-X$ \\
5 & $V V 3-X, L V 2-T$ \\
6 & $V V 3-P, L V 2-X$ \\
7 & $V V 3-X, L V 2-P$ \\
8 & $V V 3-T, L V 2-P, L V 3-F_{t}$ \\
9 & $V V 3-P, L V 2-T, L V 3-F_{t}$ \\
10 & $V V 3-T, L V 2-X, L V 3-F_{t}$ \\
11 & $L V 2-X$ \\
12 & $V V 1-T_{c}, V V 3-T, L V 2-P$ \\
13 & $V V 1-T_{c}, V V 3-P, L V 2-T$ \\
14 & $V V 1-T_{c}, L V 2-X$ \\
15 & $L V 2-X, L V 3-F_{t}$ \\
16 & $L V 2-F_{t}, L V 3-X$ \\
17 & $L V 2-F_{s}, L V 3-F_{t}$ \\
18 & $L V 2-F_{w}, L V 3-F_{t}$ \\
19 & $L V 2-X, L V 3-F_{s}$ \\
20 & $L V 2-F_{s}, L V 3-X$ \\
\hline
\end{tabular}

Table 8.4: Initial structures for evaporator MIDAOPT case study

\subsubsection{Results}

When the set of case studies was run initially, the MINLP algorithm located 14 different structures from 20 starting points. The large number of solutions located was initially attributed to the termination criterion of the MINLP algorithm. Therefore the study was rerun with an extended termination criterion, where the MINLP terminated if the algorithm generated two successive feasible NLP subproblems with objective functions worse than the best subproblem objective, rather than terminating after a single worsening in objective function. These results are presented in table 8.5. The table lists the final structure from application of the 
MIDAOPT method to the starting point, and the corresponding objective function. The next columns list the number of major iterations carried out during solution, and the number of feasible subproblems during solution. From brief examination of the results, the best solution located from the twenty starting points is the multiloop structure of $V V 3-P, L V 2-T$ with an objective function of $£ 448,900$. Note that this corresponds to the optimal structure located in the MILP evaporator case study, which suggests that this is a good solution. It cannot be stated that this is the optimal solution of the problem without examining all 689 potential structures. However, for the purposes of this discussion, this structure will be treated as the "optimal" structure for this problem. An X in the Optimal column indicates a sub-optimal solution, and a tick indicates an "optimal" solution. Finally, the last column lists the number of the major iteration when the MILP master predicted an objective function worse than the "optimal" objective function. This is an indicator of a nonconvex problem, as the master objective function is an upper bound on the subproblem objective functions for a convex problem (Kocis and Grossmann, 1988). An N/A indicates that the master problem did not reveal nonconvexity.

Although the MIDAOPT failed to locate the optimal structure consistently, these results should not be dismissed out of hand. The large number of solutions is due to the limitations of the OA/ER/AP algorithm (discussed later) where variation in economics between the optimal and suboptimal solutions is small. In fact for this study, there is a variation of only $1.2 \%$ in the objective functions between the optimal structure and the worst structure located. This indicates that although the optimal structure is not always located, the method does consistently find "good" solutions. This suggests that for problems with very small variation in economics between optimal and suboptimal structures, the MIDAOPT technique cannot differentiate easily between these structures, and tends to terminate as soon as a "good" structure is located.

It is also instructive to compare the MIDAOPT results to those of chapter 6 where the system formed the basis of an MILP case study. In general the results of the MIDAOPT analysis showed better performance than those for the linear analysis. The reasons for this will be discussed by considering two specific cases: In the linear analysis, the open loop structure was infeasible while the nonlinear analysis indicated that a feasible open loop solution existed, and the linear $V V 3, L V 2, T, P$ 


\begin{tabular}{|c|c|c|c|c|c|c|}
\hline & $\begin{array}{c}\text { Final } \\
\text { structure }\end{array}$ & $\begin{array}{c}\text { Objective } \\
\text { function } \\
(1,000 \ell)\end{array}$ & $\begin{array}{c}\text { Major } \\
\text { Iterations }\end{array}$ & $\begin{array}{c}\text { Feasible } \\
\text { NLPs }\end{array}$ & Optimal & $\begin{array}{c}\text { Nonconvex } \\
\text { Iterations }\end{array}$ \\
\hline 1 & open loop & 444.9 & 3 & 3 & $\mathrm{X}$ & N/A \\
2 & $L V 3-P$ & 445.0 & 3 & 4 & $\mathrm{X}$ & 3 \\
3 & $V V 3-P, L V 2-T$ & 448.9 & 2 & 3 & $\sqrt{ }$ & N/A \\
4 & $L V 2-F_{t}$ & 448.0 & 6 & 7 & $\mathrm{X}$ & 3 \\
5 & $L V 2-F_{t}$ & 448.0 & 7 & 8 & $\mathrm{X}$ & 3 \\
6 & $L V 2-F_{t}$ & 448.0 & 7 & 7 & $\mathrm{X}$ & 4 \\
7 & $L V 2-F_{t}$ & 448.0 & 5 & 6 & $\mathrm{X}$ & 2 \\
8 & $L V 2-F_{t}$ & 448.0 & 5 & 6 & $\mathrm{X}$ & 3 \\
9 & $V V 3-P, L V 2-T$, & 447.2 & 2 & 3 & $\mathrm{X}$ & $\mathrm{N} / \mathrm{A}$ \\
& $L V 3-F_{t}$ & & & & & \\
10 & $V V 3-F_{s}$ & 443.3 & 3 & 4 & $\mathrm{X}$ & 2 \\
11 & $L V 3-P$ & 445.0 & 3 & 4 & $\mathrm{X}$ & 2 \\
12 & $L V 3-P$ & 445.0 & 3 & 4 & $\mathrm{X}$ & 3 \\
13 & $V V 3-T$ & 445.4 & 4 & 5 & $\mathrm{X}$ & 4 \\
14 & $L V 3-P$ & 445.0 & 3 & 4 & $\mathrm{X}$ & 2 \\
15 & $L V 2-F_{t}$ & 448.0 & 5 & 6 & $\mathrm{X}$ & 1 \\
16 & $L V 2-F_{t}, L V 3-X$ & 446.9 & 2 & 3 & $\mathrm{X}$ & 2 \\
17 & $L V 2-F_{s}, L V 3-F_{t}$ & 445.9 & 2 & 3 & $\mathrm{X}$ & $\mathrm{N} / \mathrm{A}$ \\
18 & $L V 2-F_{t}$ & 448.0 & 5 & 6 & $\mathrm{X}$ & 1 \\
19 & $L V 2-F_{t}$ & 448.0 & 5 & 6 & $\mathrm{X}$ & 1 \\
20 & $L V 2-F_{s}, L V 3-X$ & 445.0 & 2 & 3 & $\mathrm{X}$ & 2 \\
\hline
\end{tabular}

Table 8.5: Shifted superstructure evaporator results

structure had a giveaway of $£ 9,000$ compared to $£ 8,300$ for the $V V 3-P, L V 2-T$ multiloop nonlinear case. On the surface these results indicate that the linear analysis is overly conservative, as it considers the best attainable performance through the perfect control assumption, whereas the nonlinear analysis considers an implemented controller on the nonlinear process. However, it must be remembered that the linear analysis considers worst case operation with respect to the disturbances, whereas the nonlinear analysis considers only a point disturbance (i.e. a single disturbance from the set of possible disturbances). Therefore it is expected that the nonlinear analysis will predict optimistic results. A simple test of this hypothesis is to vary the disturbance phase angles in closed loop simulations and test if any operating constraint violations occur. If constraint violations occur, then this implies that a greater constraint back off is required and that the current economics 
are optimistic. This test was carried out for both of the above cases at their optimal operating points using SPEEDUP, and it was found that both the minimum product concentration and minimum $V V 3$ pressure constraint were violated during operation when the disturbances were out of phase. As was indicated in chapter 6 , these are the most economically significant constraints for the evaporator, implying that the nonlinear economics of these processes are significantly underestimated by not carrying out a worst case analysis. This means that the linear analysis retains its validity, and is more likely to locate the best structure, as it incorporates worst case analysis. Thus, it is quite possible the optimal MIDAOPT structure would change if worst case analysis was included in the problem description.

As the "optimal" solution is located only once from 20 starting points, and twelve alternate suboptimal solutions are located, it can be concluded that the problem shows significant nonconvexity. This conclusion is based on the fact that the OA/ER/AP algorithm is guaranteed to locate the optimal solution from all starting points for a convex problem, and is confirmed by the master problem cutting off the optimum solution before termination in all bar four cases (an indicator of nonconvexity).

The large number of solutions generated for this problem contradicts the results obtained for the shifted superstructure in the froth flotation circuit, when the global optimum was located from all feasible starting points. By re-examining the flotation problem, it may be seen that these results were because of the specific nature of the problem, rather than a reflection of the shifted superstructure. In the flotation study, the strongest economic improvement was associated with employing the feed water to control the percentage water in the feed. Therefore, the MILP master always made this controller active. A much weaker economic benefit was associated with the use of the air factor to control either product grade or product recovery. However, as there was no cost associated with the controllers, the master could always improve the objective function by choosing to make one of the air factor controllers active. If the suboptimal air factor controller was chosen first, the next iteration would be expected to choose the optimal controller. Therefore it would be expected that the MIDAOPT would succeed in locating the optimum configuration in all cases for the particular case of the flotation circuit problem. Note that this does not negate the benefits of using the MIDAOPT to solve the flotation circuit 
problem, as it still solved the problem significantly faster than explicit enumeration.

In spite of locating consistently good solutions, obtaining 12 answers from 20 starting points is not ideal performance. The question which is immediately raised is: why did the OA/ER/AP algorithm perform poorly for this nonconvex problem? A qualitative answer to this question may be developed from examining figure 8.1, which shows the plane of continuous variables $x 1$ and $x 2$, with a single nonconvex constraint and a set of subproblem solutions 1 to 7 (corresponding to optimal continuous solutions for a fixed integer vector), where point 2 is the optimal integer solution. Consider starting the algorithm from point 1 . Linearising the constraint results in the dashed line. An integer solution which lies below the linearised constraint will be referred to as an interior point, and a solution above the line as an exterior point. This distinction is made to distinguish between points which will activate the penalty function (exterior), and those which will not (interior) when located by the master. Remembering that the penalty weight for violating a linearised constraint is set to 1000 times the Lagrange multiplier of the nonlinear constraint (Viswanathan and Grossmann, 1990), and noting that the Lagrange multiplier of the linearised constraint is expected to be of a similar magnitude to the nonlinear Lagrange multiplier, the following argument may be posed. For the master to locate optimum solution (2) will require a violation of the linearised constraint, at a cost roughly proportional to 1000 times the Lagrange multiplier times the magnitude of the constraint violation. The expected improvement in the objective function relative to the relaxed MILP solution is roughly proportional to the Lagrange multiplier times the magnitude of the constraint violation (i.e. a large penalty is exacted for violating the constraint). Clearly if there is an interior point which is "close" to the optimal solution, such as point 4 , it is more likely to be chosen as the next search point, as the degradation in the objective function relative to the relaxed solution is roughly proportional to the Lagrange multiplier times the distance of the solution from the constraint. Based on this argument, nonconvex points $(2,3)$ are only likely to be chosen if they are close to the interior region and there are no interior points which are also close to the active constraints. The evaporator case study clearly does not meet these conditions, as there are many points which have similar objective functions, and the benefits of changing integer solutions are not dramatic compared to the cost of violating the constraints. Wording the argument in a slightly dif- 
ferent form, the OA/ER/AP algorithm is inadequate for solving problems where modifying the integer vector results in changes in objective function similar to the improvements expected from violating the constraints.

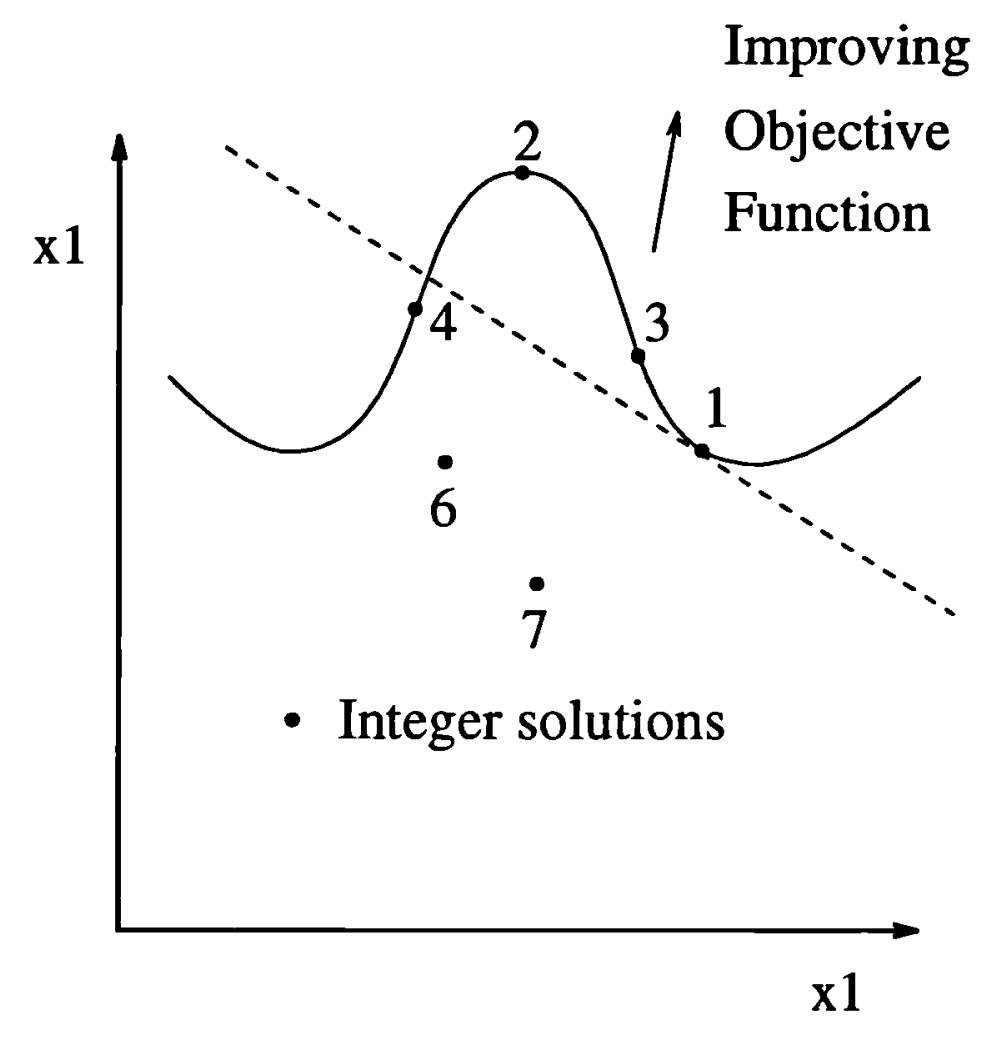

Figure 8.1: Nonconvex constraints in MINLP problems

Based on these results, it can be concluded that the shifted superstructure MIDAOPT approach can be used for the control structure selection to obtain consistently "good" structures. However, the results obtained should be used with caution as they are not guaranteed to be optimal because of the problem nonconvexity and the lack of worst case disturbance analysis.

\subsubsection{Full superstructure results}

From the above discussion, it is clear that the nonconvexity of the problem is the largest stumbling block for the method. The nonconvexity may arise from two sources: the use of nonlinear integer vectors, and from the path constraints (as no attempt has been made to identify the convexity properties of these constraints). It is therefore appropriate to test whether removing one of these sources of nonconvexity could improve the MIDAOPT performance significantly. The path 
constraints cannot be eliminated, but the nonlinearity of the integer variables may be removed at the cost of larger subproblems by using the full controller superstructure. Therefore the case studies were run again using this representation to test if the MIDAOPT performance improved.

The results for the full superstructure case are summarised in table 8.6. In this case the "optimal" solution is the $V V 3-T, L V 2-F_{t}$ multiloop structure with an objective of $£ 449,200$. Note that there is some slight variation in the objective functions compared to the shifted superstructure case. This is because the original controller representation was used in the full superstructure rather than the scaled version used in the shifted superstructure. The differences between the cases are due to discrepancies in controller parameter bounds from the different descriptions. However, the differences are small: the largest variation occurs for the $V V 3-P, L V 2-$ $T$ structure (case 3 ), with only a $0.15 \%$ change in the objective function between the shifted and full superstructure solutions. These variations have a negligible effect on the conclusions for the problem. To maintain consistency with the shifted superstructure case, the extended termination criterion was used for all runs.

Again, it can be seen that the problem is nonconvex, as the optimal solution is only found in two cases, and 10 solutions are found from 19 starting points. This shows that the path constraints cause significant nonconvexity that cannot be eliminated from the problem description. However, it appears that the convexity of the problem is somewhat improved, as the master predicts nonconvexity in only three cases, rather than the sixteen cases for the shifted superstructure. The full superstructure also resulted in the location of a slightly improved optimal structure: the $V V 3-T, L V 2-F_{t}$ multiloop configuration. However, the performance of the method is still not ideal, generating 10 solutions from 19 starting points. From this it is concluded that the quality of MIDAOPT solutions could be significantly improved through the use of more robust optimisation technique that can handle the nonconvexities due to the path constraints.

Besides the nonconvexity problem, another argument against the frequent use of MIDAOPT methods is the time required for solution of even small problems. The time required for a single case was typically 24 to 36 hours for the shifted superstructure, and 2 to 4 days for the full superstructure, of dedicated Sparc II time, where most of the time $(99 \%+)$ was spent on evaluation of the NLP subproblems. 


\begin{tabular}{|c|c|c|c|c|c|c|}
\hline & $\begin{array}{c}\text { Final } \\
\text { structure }\end{array}$ & $\begin{array}{c}\text { Objective } \\
\text { function } \\
(1,000 £)\end{array}$ & $\begin{array}{c}\text { Major } \\
\text { Iterations }\end{array}$ & $\begin{array}{c}\text { Feasible } \\
\text { NLPs }\end{array}$ & Optimal & $\begin{array}{c}\text { Nonconvex } \\
\text { Iterations }\end{array}$ \\
\hline 1 & $V V 3-P, L V 2-T$ & 448.2 & 3 & 4 & $\mathrm{X}$ & N/A \\
\hline 2 & $V V 3-T, L V 2-P$ & 444.2 & 2 & 3 & $\mathrm{X}$ & N/A \\
\hline 3 & $V V 3-P, L V 2-T$ & 448.2 & 2 & 3 & $\mathrm{X}$ & N/A \\
\hline 4 & $V V 3-T, L V 2-X$ & 444.4 & 2 & 3 & $\mathrm{X}$ & N/A \\
\hline 5 & $V V 3-T, L V 2-F_{t}$ & 449.2 & 4 & 5 & $\sqrt{ }$ & 4 \\
\hline 6 & $L V 2-F_{t}$ & 448.0 & 5 & 6 & $X$ & 4 \\
\hline 7 & $V V 3-P, L V 2-T$ & 448.2 & 3 & 4 & $\mathrm{X}$ & N/A \\
\hline 8 & $V V 3-P, L V 2-T$ & 448.2 & 3 & 4 & $\mathrm{X}$ & N/A \\
\hline 9 & $\begin{array}{c}V V 3-P, L V 2-T \\
L V 3-F_{t}\end{array}$ & 446.8 & 2 & 3 & $\mathrm{X}$ & N/A \\
\hline 10 & $\begin{array}{c}V V 3-T, L V 2-X \\
L V 3-F_{t}\end{array}$ & 446.4 & 2 & 3 & $\mathrm{X}$ & N/A \\
\hline 11 & $V V 3-P, L V 2-T$ & 448.2 & 3 & 4 & $\mathrm{X}$ & $\mathrm{N} / \mathrm{A}$ \\
\hline 12 & \multicolumn{6}{|c|}{ Failed during solution } \\
\hline 13 & $\begin{array}{c}V V 1-T_{c}, V V 3-P \\
L V 2-T\end{array}$ & 447.1 & 2 & 3 & $\bar{X}$ & $\mathrm{~N} / \mathrm{A}$ \\
\hline 14 & $V V 3-P, L V 2-T$ & 448.2 & 3 & 4 & $\mathrm{X}$ & N/A \\
\hline 15 & $V V 3-P, L V 2-T$ & 448.2 & 6 & 4 & $\mathrm{X}$ & N/A \\
\hline 16 & $V V 3-P, L V 2-T$ & 448.2 & 3 & 4 & $\mathrm{X}$ & $\mathrm{N} / \mathrm{A}$ \\
\hline 17 & $L V 2-F_{s}, L V 3-F_{t}$ & 446.0 & 2 & 3 & $\mathrm{X}$ & N/A \\
\hline 18 & $L V 2-F_{w}, L V 3-F_{t}$ & 446.0 & 2 & 3 & $\mathrm{X}$ & $\mathrm{N} / \mathrm{A}$ \\
\hline 19 & $V V 3-T, L V 2-F_{t}$ & 449.2 & 3 & 4 & $\sqrt{ }$ & $\mathrm{N} / \mathrm{A}$ \\
\hline 20 & $V V 3-T, L V 2-F_{w}$ & 448.4 & 5 & 6 & $\mathrm{X}$ & 5 \\
\hline
\end{tabular}

Table 8.6: Full superstructure evaporator results

This is an extremely long time to determine control structures for a 4 by 8 system, and makes the method inappropriate for routine use. As both the dynamic optimisation techniques and the SQP methods are relatively well developed technologies, the only practical solutions to this problem are to wait for faster computers or to develop efficient parallel algorithms for the problem solution. In addition, the approach to this problem has considered only a point disturbance (that is a single disturbance from the set of possible process disturbances). As has already been noted, for a practical control analysis, a worst case design must be considered to ensure that the best controller is selected, rather than the controller which handles an isolated disturbance best. Addition of such analyses would be expected to increase the sub- 
problem solution time by at least an order of magnitude (Walsh, 1992), further suggesting that the use of nonlinear dynamic analysis for control structure selection using existing techniques is only practical for small processes with relatively well behaved dynamics.

\subsubsection{Full superstructure case - reduced weights}

A final full superstructure run was carried out to test the hypothesis proposed in section 8.2.1 for the poor MINLP performance. This hypothesis argued that the OA/ER/AP algorithm will preferentially examine interior integer solutions close to the relaxed optimum rather than exterior integer solutions due to the penalisation of these solutions. To test this theory, an obvious modification to the algorithm is to reduce the penalty weights, and rerun the problem to determine if this has a significant effect on MINLP performance. To satisfy the linear approximation to an exact penalty function used by the augmented penalty algorithm (Zhang et al., 1985; Viswanathan and Grossmann, 1990), the penalty weights must be strictly greater than the Lagrange multipliers of the optimal solution of the NLP subproblem. Therefore, to test the hypothesis, the problem was rerun for the full superstructure with penalty weights reduced from 1000 times the Lagrange multipliers to 1.1 times the Lagrange multipliers. If the hypothesis is correct, then one of two outcomes is expected:

1. Little or no change in the results, implying that the MINLP search has remained in the $\mathrm{OA} / \mathrm{ER}$ feasible region in preference to activating the nonconvex penalty function for both weight cases. This implies that a large number of interior points are close to the relaxed optimum, allowing the master to choose interior integer solutions with less objective function degradation than the penalised degradation for exterior solutions.

2. Improvement in the solutions, implying that MINLP search has activated the penalty functions to locate nonconvex solutions, and the reduced penalty weight has allowed deeper forays into the exterior region.

The results of this set of runs are summarised in table 8.7.

Comparing these results with those for the full superstructure with normal weights (table 8.6), it can be seen that the only differences between the two cases 


\begin{tabular}{|c|c|c|c|c|c|c|}
\hline & $\begin{array}{c}\text { Final } \\
\text { structure }\end{array}$ & $\begin{array}{l}\text { Objective } \\
\text { function } \\
(1,000 £)\end{array}$ & $\begin{array}{c}\text { Major } \\
\text { Iterations }\end{array}$ & $\begin{array}{c}\text { Feasible } \\
\text { NLPs }\end{array}$ & Optimal & $\begin{array}{c}\text { Nonconvex } \\
\text { Iterations }\end{array}$ \\
\hline 1 & $V V 3-P, L V 2-T$ & 448.2 & 3 & 4 & $\bar{X}$ & N/A \\
\hline 2 & $V V 3-T, L V 2-P$ & 444.2 & 2 & 3 & $X$ & $\mathrm{~N} / \mathrm{A}$ \\
\hline 3 & $V V 3-P, L V 2-T$ & 448.2 & 2 & 3 & $\mathrm{X}$ & $\mathrm{N} / \mathrm{A}$ \\
\hline 4 & $V V 3-T, L V 2-X$ & 444.4 & 2 & 3 & $\mathrm{X}$ & $\mathrm{N} / \mathrm{A}$ \\
\hline 5 & $V V 3-T, L V 2-F_{t}$ & 449.2 & 4 & 5 & $\sqrt{ }$ & 4 \\
\hline 6 & $L V 2-F_{t}$ & 448.0 & 5 & 6 & $\mathrm{X}$ & 4 \\
\hline 7 & $V V 3-P, L V 2-T$ & 448.2 & 3 & 4 & $\mathrm{X}$ & N/A \\
\hline 8 & $V V 3-P, L V 2-T$ & 448.2 & 3 & 4 & $\mathrm{X}$ & N/A \\
\hline 9 & $\begin{array}{c}V V 3-P, L V 2-T \\
L V 3-F_{t}\end{array}$ & 446.8 & 2 & 3 & $\mathrm{X}$ & $\mathrm{N} / \mathrm{A}$ \\
\hline 10 & $\begin{array}{c}V V 3-T, L V 2-X \\
L V 3-F_{t}\end{array}$ & 446.4 & 2 & 3 & $\mathrm{X}$ & $\mathrm{N} / \mathrm{A}$ \\
\hline 11 & $V V 3-P, L V 2-T$ & 448.2 & 6 & 4 & $\mathrm{X}$ & N/A \\
\hline 12 & \multicolumn{6}{|c|}{ Failed during solution } \\
\hline 13 & $\begin{array}{c}V V 1-T_{c}, V V 3-P \\
L V 2-T\end{array}$ & 447.1 & 2 & 3 & $\mathrm{X}$ & N/A \\
\hline 14 & $V V 3-P, L V 2-T$ & 448.2 & 3 & 4 & $\mathrm{X}$ & $\mathrm{N} / \mathrm{A}$ \\
\hline 15 & $V V 3-P, L V 2-T$ & 448.2 & 6 & 4 & $\mathrm{X}$ & $\mathrm{N} / \mathrm{A}$ \\
\hline 16 & $V V 3-P, L V 2-T$ & 448.2 & 3 & 4 & $\mathrm{X}$ & $\mathrm{N} / \mathrm{A}$ \\
\hline 17 & $L V 2-F_{s}, L V 3-F_{t}$ & 446.0 & 2 & 3 & $\mathrm{X}$ & $\mathrm{N} / \mathrm{A}$ \\
\hline 18 & $L V 2-F_{w}, L V 3-F_{t}$ & 446.0 & 2 & 3 & $\mathrm{X}$ & N/A \\
\hline 19 & $V V 3-T, L V 2-F_{t}$ & 449.2 & 3 & 4 & $\sqrt{ }$ & N/A \\
\hline 20 & $\begin{array}{c}V V 3-T, L V 2-F_{t} \\
L V 3-X\end{array}$ & 448.0 & 4 & 5 & $\mathrm{X}$ & 3 \\
\hline
\end{tabular}

Table 8.7: Full superstructure results — reduced weights

are:

- For run 11 , the reduced weight case examines three more infeasible integer solutions

- For run 20 , the algorithm locates a new structure, the $V V 3-T, L V 2-F_{t}, L V 3-$ $X$ structure in one less major iteration, with an objective function $£ 400$ per year worse than the normal weight case

Aside from the anomalous results in run 20 , there is little change between the cases, implying that the first possible outcome has occurred - that is interior points have been chosen for both cases without activating the penalty functions. Considering 
both cases of run 20 iteration by iteration, it can be seen that this result is consistent with the expected behaviour of the algorithm. Both cases choose the same structure in the first major iteration, with an objective function of $£ 441,500$ per annum. However in the second major iteration, the normal weight case locates a solution with an objective function of $£ 446,000$, and the reduced weight case locates a solution with an objective function of $£ 448,000$. On the next iteration, the normal weight case locates a slightly better solution of $£ 448,400$. This solution is not located by the reduced weight case, indicating that it now lies in the penalised region. That is, the reduced weight case locates a good solution earlier in the search, but its linearisation acts to cut off the better solution located by the full weight case later in the search. As the reduced weight case fails to locate this solution, this serves to confirm the hypothesis that the penalty function prevents searches in the exterior region if there are "close" interior solutions. With the anomaly confirming the hypothesis, these results strongly suggest that the hypothesis for the poor performance of the OA/ER/AP is correct, and that these arguments can be extended to general nonconvex problems with dense interior point distributions.

\subsection{Summary and conclusions}

In summary, the MIDAOPT approach for multiloop controller selection was applied to two case studies. The first case study showed promising results, however these were shown to be a function of the problem rather than the solution method. The second case study demonstrated that the OA/ER/AP method performs poorly for MIDAOPT because of the nonconvexity of the problem. It was shown that this performance is due to the difficulty of the master being able to pick points in the penalised region when the system has large numbers of feasible interior points with small objective function degradations relative to the penalised degradations. In spite of the limitations highlighted in this chapter, it was concluded that the MIDAOPT technique can be used for control structure selection as it is expected to locate consistently "good" (but not optimal) structures. However further case studies should be carried out to further validate this technique, and any solutions generated should be employed with care because of the above limitations. 


\section{Chapter 9}

\section{Summary and conclusions}

Recognising recent concern with the interactions between steady state process design and process dynamics, this thesis developed systematic approaches to the problem of economically optimal control structure selection for design purposes. Preliminary analysis of the general combined steady state and dynamic design problem indicated that it is impractical to solve this problem using existing techniques. Therefore, based on a steady state bounding of the dynamic process economics demonstrated in chapter 2 , the problem was decomposed into the more tractable subproblems of steady state process design to generate alternative flowsheets, and analysis of the optimal dynamic economics of a specified flowsheet. As steady state process design is a well understood problem, the remainder of the thesis concentrated on the systematic analysis of dynamic economics.

Examination of dynamic economics showed that they are dependent on the process controller employed, and that the performance of the controller is limited by the control structure employed. Two important, related aspects of control structure performance were identified:

- The limits of control that can be exerted by the manipulated variables on the measured (controlled) variables (control quality)

- The level of attainment of the control objectives by the control structure (control aims)

It was also noted that the process control objectives are closely related to the steady state process economics and the active operating constraints. A review of previous approaches to the problem of control structure selection indicated that a significant contribution could be made by developing systematic methods for selecting the economically optimal process control structure. Therefore, this was made the main aim of this thesis. As the thesis considers the problem for design purposes, the problem is to choose the economically optimal control structure from the set of potential 
control structures. It was shown that dynamic economics of the control structures are dependent on both aspects of the control structure performance identified above.

The review of previous approaches revealed several deficiencies, noted below:

1. Large numbers of alternative structures are generated.

2. Process economics are used only to select control objectives, while ranking of alternative control structures is carried out on the basis of heuristics or controllability analyses, neither of which are related directly to economic performance.

3. Neither the relationship between the active process constraints and control objectives nor the relationship between the control objectives and the measured variables were dealt with clearly.

Based on these deficiencies, it was determined that control structure selection techniques should have the potential to locate all possible process control structures and, to avoid the combinatorial problem of examination of all of these structures, they should employ assessment of dynamic economics to guide the search, ensuring that only "good" structures are generated. Both nonlinear and combined linear/nonlinear dynamic analysis approaches to control structure selection were examined.

\subsection{Linear dynamic economics}

The first stage in the combined linear/nonlinear analysis was to develop a linear dynamic economic analysis for a specific control structure. The procedure is as follows:

1. Develop a nonlinear dynamic design model of the process and carry out a steady state optimisation.

2. Linearise the process model, choosing disturbance and manipulated variables as inputs, and the constraint slack and measured variables as outputs.

3. Given disturbance magnitude and frequency information, use frequency response calculations and perfect control assumptions to obtain a linear estimate of the maximum constraint back off. 
4. Generate a steady state linear program approximation of the nonlinear dynamic model, and augment it with the maximum constraint back offs. Solve to determine a linear estimate of the dynamic economics.

5. Carry out a linear controllability analysis to determine the attainability of perfect control.

A case study demonstrated that the technique can select economically optimum process designs and control structures where conventional steady state economics and linear controllability analysis did not give clear results.

There are three areas where further research could significantly extend the application of this technique. Firstly, the technique uses a perfect control assumption that supplies a limiting value of the attainable dynamic economics. It would be preferable to calculate a practical value of the dynamic economics, rather than the optimistic values predicted by perfect control analysis. One possible approach to this problem could be to consider generic controllers (for example the class of multiloop PI controllers or the class of LQG controllers, etc.) and determine the limiting performance that can be attained with these generic controllers. This raises the problem of describing generic controller performance without generating a complex optimisation problem and negating the simplicity of linear analysis.

The two remaining areas for further research are the related areas of controllability and process uncertainty. Currently these aspects are addressed by functional controllability analysis after the perfect control dynamic economics have been assessed. However, to make the analysis a more effective technique for control structure selection, it would be desirable to examine these aspects simultaneously with the economic analysis. One approach would be to include pass/fail analysis of control structure robustness based on the internal model control approaches of Morari et al. (see chapter 3), or the singular value based robustness approach of Reeves et al. (1991). In this case a robustness specification is included in the problem input data, and control structures are rejected if they do not meet the robustness criterion, in the same fashion as structures with an infeasible constraint back off.

A further extension to the technique is the assessment of dynamic economics for structures with excess manipulated variables, or structures where several measured variables are used as estimators of a variable that cannot be measured 
directly.

\subsection{MILP control structure selection}

The linear dynamic economic analysis is used as the basis for a hybrid mixed integer linear programming (MILP) formulation of the control structure problem. A linear model of the process is generated in the same fashion as the dynamic economic analysis, except that all of the potential manipulated and measured variables are included in the linearisation. An MILP approximation to the perfect control problem is developed from the linear model, using integer variables to describe the possible perfect control relationships. Solution of the MILP generates alternative control structures based on optimistic estimates of the perfect control dynamic economics. A full perfect control dynamic economic analysis is carried out for each structure generated by the MILP, and these economics are used to update the entry costs in the branch and bound MILP algorithm. This defines the pruning of the search tree in the algorithm in terms of the exact dynamic economics, rather than the optimistic economics generated by the MILP solution.

The method was demonstrated on two case studies, and it was shown to be an effective technique for control structure selection, although it should be possible to improve the speed of solution by improving the LP representation and modifications to the MILP formulation and search termination criterion. It was noted that the most important aspect of control structure economics is the marginal cost of the instrumentation (sensors and actuators), where the marginal cost is given by the instrumentation cost minus the improvement in economics obtained from employing the instrumentation. Finally it was noted that the technique reversed conventional control structure selection procedures, as the control objectives were determined implicitly during the solution procedure, rather than defining control objectives and then generating control structures to attain these objectives.

Several extensions of this approach are suggested, starting with multiperiod MILP representations to generate the optimal control structure for a number of disturbance conditions, where each period in the MILP corresponds to a different disturbance condition, and the integer variables and constraint back offs are the common variables between the periods. A second obvious multiperiod extension is to 
generate control structures for multiregime processes, where each period represents one of the plant operating regimes. This approach can be used to generate either a single overall control structure to be applied to all regimes, or control structures for each regime, using appropriate integer constraints to ensure that each actuator and sensor is paid for only once (i.e. a sensor or actuator used in one period can be employed in the other periods at no additional cost). It may also be possible to use the multiperiod analysis to examine process uncertainty, although it is not clear how this should be implemented.

Using the ideas of sensed variable trees (Govind and Powers, 1982), it may be possible to use the MILP approach to select measurements that infer unmeasurable controlled variables. This would require identification of all of the possible measured variables and constraints (potential control objectives) on the process. The structural Jacobian of the DAE model could then be used to generate the integer logic describing relationships between unmeasurable slacks and measurable variables. This logic describes an unmeasurable variable as inferable if there is a functional relationship between it and a set of variables that can be measured directly or are themselves inferable (i.e. use the functional relationship to calculate the unmeasurable variable).

\subsection{MIDAOPT control structure selection}

Chapters 7 and 8 detailed a nonlinear dynamic approach to the control structure selection problem. As this was a preliminary foray into nonlinear control structure selection, it was decided to consider the selection of multiloop PI controllers. The problem was posed as a DAE model of the process plant, with an associated superstructure of PI controllers in a form amenable to solution by a combination of dynamic optimisation and mixed integer nonlinear programming (MINLP) techniques. Application of the technique to case studies demonstrated that existing MINLP methods are not capable of handling the nonconvexities introduced by the nonlinear constraints in the dynamic optimisation subproblems and that worst case disturbance analysis should be included in the problem description to ensure that the control structure economics are not underestimated. In spite of these limitations, promising results were obtained for the case studies and it was 
concluded that the MIDAOPT technique can be used for control structure selection as it is expected to locate consistently "good" (but not optimal) structures. However further case studies should be carried out to further validate this technique, and any solutions generated should be employed with care because of the above limitations.

Given the limitations of the MIDAOPT technique, the following ideas are suggested as alternative avenues for examination of the nonlinear control structure problem. Firstly, some promising initial research has been carried out in the area of nonlinear controllability indicators (Biss, 1991) that are nonlinear analogues of linear controllability indicators. It may be possible to exploit these ideas to obtain a nonlinear analogue to linear perfect control and use this as the basis of a control structure selection algorithm similar to the MILP approach described previously. The other alternative is to solve the problem as a large dynamic optimisation using an embedded description of the controller superstructure where the structural parameters are continuous parameters (Nishida and Ichikawa, 1975; Sargent and Gaminibandara, 1976), as dynamic optimisation techniques were demonstrated to be more reliable than the MINLP techniques in the case studies considered.

\subsection{Summary}

This thesis has demonstrated that it is possible to select economically optimal process control structures in an efficient manner. However, it also demonstrated that there is a sparsity of appropriate techniques for these problems and that there are significant contributions still to be made investigating both techniques for and approaches to the control structure selection problem. 


\section{References}

R.K. Ahuja, T.L. Magnanti, and J.B. Orlin. Network flows. In G.L. Nemhauser, A.H.G. Rinnooy Kan, and M.J. Todd, editors, Handbooks in Operations Research and Management Science. Volume 1: Optimization. Elsevier Science Publishers B.V., North Holland, 1989.

Y. Arkun and G. Stephanopoulos. Studies in the synthesis of control structures for chemical processes: Part IV. Design of steady-state optimizing control structures for chemical process units. AIChE J, 26(6):p975, 1980.

Y. Arkun and G. Stephanopoulos. Studies in the synthesis of control structures for chemical processes. Part V: Design of steady-state optimizing control structures for integrated chemical plants. AIChE J, 27(5):p779, 1981.

G.W. Barton and J.D. Perkins. Experiences with SPEEDUP in the process industries. Computers and Chemical Engineering, 66(8):p408, 1988.

G.W. Barton, W.K. Chan, and J.D. Perkins. Interactions between process design and process control: The role of open loop indicators. Journal of Process Control, 1:p161, 1991.

F.L. Bauer. Optimally scaled matrices. Numer. Math., 5:p73, 1963.

E.M.L. Beale. Integer programming. In The State of the Art in Numerical Analysis, page 409. Academic Press, 1977.

J.F. Benders. Partitioning procedures for solving mixed-variables programming problems. Numerische Mathematik, 4:p238, 1962.

L.T. Biegler. Strategies for simultaneous solution and optimization of differential algebraic systems. In Proceedings of the Third International Conference on Foundations of Computer-Aided Process Design. CaChE-Elsevier, 1990.

D. Biss. Controllability Analysis for Non-linear Systems. Technical report, The Centre for Process Systems Engineering, Imperial College, University of London, 1991. 
P.S. Buckley. Techniques of process control. Wiley, New York, 1964.

M.B. Carver. The choice of algorithms in automated method of lines solution of partial differential equations. In Numerical Methods for Differential Systems - Recent Developments in Algorithms, Software and Applications, L. Lapidus and W.E. Schiesser (Eds.). Academic Press, London, 1976.

C.L. Chen. A class of successive quadratic programming methods for flowsheet optimisation. PhD thesis, Dept. Chem. Eng., Imperial College, University of London, 1988.

J.E. Cuthrell and L.T. Biegler. On the optimization of differential-algebraic process systems. AIChE J., 33(8):p282, 1987.

E.J. Davison. Connectability and structural controllability of composite systems. Automatica, 13:p109, 1977.

J.M. Douglas. Conceptual design of chemical processes. McGraw-Hill, Singapore, 1988.

R. Duran and I.E. Grossmann. A mixed integer nonlinear programming approach for process systems synthesis. AIChE Journal, 32(4):p592, 1986a.

R. Duran and I.E. Grossmann. An outer-approximation algorithm for a class of mixed integer nonlinear programs. Math. Programming, 36:p307, 1986 b.

T.F. Edgar and D.M. Himmelblau. Optimization of chemical processes. McGrawHill, 1988.

J.H. Fewings, P.J. Slaughter, E. Manlapig, and A.J. Lynch. Experiences with process control of the chalcopyrite flotation circuit at Mount Isa Mines Limited. In 13th Int. Min. Proc. Congress, Warsaw, page 1540, 1979.

W.R. Fisher, M.F. Doherty, and J.M. Douglas. The interface between design and control: 1. Process controllability. IEC Res., 27(4):p597, 1988a.

W.R. Fisher, M.F. Doherty, and J.M. Douglas. The interface between design and control: 2. Process operability. IEC Res., 27(4):p606, 1988b. 
W.R. Fisher, M.F. Doherty, and J.M. Douglas. The interface between design and control: 3. Selecting a set of controlled variables. IEC Res., 27(4):p611, 1988c.

D.W. Fuerstenau, editor. Froth flotation : 50th anniversary volume. American Institute of Mining, Metallurgical and Petroleum Engineers, New York, 1962.

C. Gannavarapu. Economic assessment in the synthesis of optimising control schemes. PhD thesis, Dept. Chem. Eng., University of Sydney, 1991.

G. Gavigan. Control: A winning strategy. IEE Review, page 191, 1991.

C.W. Gear. Simultaneous numerical solution of differential-algebraic equations. IEEE Transactions on Circuit Theory, CT-18:89, 1971.

A.M. Geoffrion. Generalised Benders decomposition. J. Optimization Theory Applic., 10:p237, 1972.

A. Georgiou and C.A. Floudas. Optimization model for generic rank determination of structural matrices. Int. J. Control, 49(5):p1633, 1989a.

A. Georgiou and C.A. Floudas. Structural analysis and synthesis of feasible control systems - theory and applications. Chem. Eng. Res. Des., 67:p600, 1989b.

A. Georgiou and C.A. Floudas. Structural properties of large scale systems. Int. J. Control, 51(1):p169, 1990.

A. Georgiou and C.A. Floudas. Rẹponse to comments on 'Structural analysis and synthesis of feasible control systems - theory and applications'. Chem. Eng. Res. Des., 70:430, 1992.

P.E. Gill, W. Murray, and M.H. Wright. Practical optimization. Academic Press, San Diego, 1981.

R. Govind and G.J. Powers. Control system synthesis strategies. AIChE Journal, 28:p60, 1982.

D. Gritsis. The dynamic simulation and optimal control of systems described by index two differential-algebraic equations. PhD thesis, Dept. Chem. Eng., Imperial College, University of London, 1990. 
I.E. Grossmann and M. Morari. Operability, resiliency and flexibility: process design objectives for a changing world. In FOCAPD II, page 931. CACHE, 1984.

I.E. Grossmann, K.P. Halemane, and R.E. Swaney. Optimization strategies for flexible chemical processes. Computers and Chemical Engineering, 7(4):p439, 1983.

I.E. Grossmann. MINLP optimization strategies and algorithms for process synthesis. In Proceedings of the third International Conference on Foundations of Computer Aided Process Design, page 105. Cache-Elsevier Amsterdam, 1990.

S.S. Jang, B. Joseph, and H. Mukai. On-line optimization of constrained multivariable chemical processes. AIChE J., 33(1):p26, 1987.

R.B. Jarvis and C.C. Pantelides. DASOLV - A differential-algebraic equation solver. User Manual, Centre for Process Systems Engineering, Imperial College, University of London, 1992.

R.D. Johnston and G.W. Barton. Design and performance assessment of control systems using singular-value analysis. IEC Res., 26:p830, 1987.

R.D. Johnston, G.W. Barton, and M.L. Brisk. Single-input single-output control system synthesis. Part 1: Structural analysis and the development of feedback control schemes. Computers and Chemical Engineering, 9(6):p547, 1985a.

R.D. Johnston, G.W. Barton, and M.L. Brisk. Single-input single-output control system synthesis. Part 2: Application of the synthesis algorithm to an integrated plant. Computers and Chemical Engineering, 9(6):p557, 1985b.

R.E. Kalman. Contributions to the theory of optimal control. Bol. Soc. Mat. Mexicana, 5:p102, 1960.

Kirk-Othmer, Concise Encyclopedia of Chemical Technology, 1985.

G.R. Kocis and I.E. Grossmann. Relaxation strategy for the structural optimization of process flowsheets. IEC Res., 26:p1869, 1987.

G.R. Kocis and I.E. Grossmann. Global optimization of nonconvex MINLP problems in process synthesis. IEC Res., 27:p1421, 1988. 
W. Lee and V.W. Weekman. Advanced control practice in the chemical process industry: A view from industry. AIChE J., 22(1):p27, 1976.

A.M. Lenhoff and M. Morari. Design of resilient processing plants I: Process design under consideration of dynamic aspects. Chem. Eng. Sci., 37(2):p245, 1982.

B. Lie and J.G. Balchen. The pairing problem in process control: A critical survey. Presented at The AIChE Annual Meeting, 1991.

M.L. Luyben and C.A. Floudas. A multiobjective optimization approach for analyzing the interaction of design and control. The 1992 IFAC Workshop on Interactions Between Process Design and Process Control, Pergammon, London, 1992.

W.L. Luyben. Process modeling, simulation and control for chemical engineers, 2nd Ed. McGraw Hill, Singapore, 1990.

A.J. Lynch, N.W. Johnson, E.V. Manlapig, and C.G. Thorne. Mineral and Coal Flotation Circuits. Elsevier N.Y., 1981.

K.J. MacCallum. Conceptual design environments - research directions and issues. In Proceedings Strategic research issues in AI in Engineering, IEE, London, 1990.

A.G.J. MacFarlane and D.F.A Jones. Vector gain. Int. Journal Control, 29:p65, 1979.

T.E. Marlin, J.D. Perkins, G.W. Barton, and M.L. Brisk. Benefits from process control: results of a joint industry-university study. J. Process Control, 1:p68, 1991.

H. Mawengkang and B.A. Murtagh. Solving nonlinear integer programs with large scale optimization software. Ann. Oper. Res., 5:p425, 1986.

M. Morari and S. Skogestad. Effect of model uncertainty on dynamic resilience. In IChemE Symp. Ser. No. 92, page p493, 1985. 
M. Morari and G. Stephanopoulos. Studies in the synthesis of control structures for chemical processes. Part II: Structural aspects and the synthesis of alternative feasible control schemes. AIChE Journal, 26(2):p232, 1980a.

M. Morari and G. Stephanopoulos. Studies in the synthesis of control structures for chemical processes. Part III: Optimal selection of secondary measurements within the framework of state estimation in the presence of persistent unknown disturbances. AIChE Journal, 26(2):p247, 1980 b.

M. Morari and E. Zafiriou. Robust process control. Prentice-Hall, New Jersey, 1989.

M. Morari, Y. Arkun, and G. Stephanopoulos. Studies in the synthesis of control structures for chemical processes. Part I: Formulation of the problem. Process decomposition and the classification of the control tasks. Analysis of the optimizing control structures. AIChE Journal, 26(2):p221, 1980.

M. Morari. Integrated plant control: A solution at hand or a research topic for the next decade. In Chemical Process Control II (T.F. Edgar and D.E. Seborg). AIChE Publications, 1981.

M. Morari. Design of resilient processing plants III: A general framework for the assessment of dynamic resilience. Chem. Eng. Sci., 38:p1881, 1983.

M. Morari. Three critiques of process control revisited a decade later. In The Shell Process Control Workshop. (M. Morari and D.M. Prett Eds). Butterworths, 1987.

K.R. Morison. Optimal control of process systems described by systems of differential-algebraic equations. PhD thesis, Dept. Chem. Eng., Imperial College, University of London, 1984.

B.A. Murtagh and M.A. Saunders. MINOS 5.0 users guide, Technical Report SOL 83-20, Stanford University, 1983.

I.M. Mutjaba and S. Machietto. Optimal control of batch distillation. In Proceedings of the 12th IMACS World Congress, 1988.

G.L. Nemhauser and L.A. Wolsey. Integer and combinatorial optimization. John Wiley and sons, New York, 1988. 
N. Nishida and A. Ichikawa. Synthesis of optimal dynamic process systems by a dynamic gradient method. Ind. Eng. Chem., Proc. Des. Dev., 14(3):p236, 1975.

N. Nishida, G. Stephanopoulos, and A.W. Westerberg. A review of process synthesis. AIChE J., 27(4):p423, 1981.

M.A. Padley. Incorporating operability measures into process synthesis. $\mathrm{PhD}$ thesis, Dept. Chem. Eng., University of Sydney, 1991.

A. Palazoglu and Y. Arkun. Studies on the design of robust chemical plants. In Process systems engineering '85, page p457. EFCE Publication series no. 40, 1985.

A. Palazoglu and Y. Arkun. A multiobjective approach to design chemical plants with robust dynamic operability characteristics. Computers and Chemical Engineering, 10(6):p567, 1986.

A. Palazoglu and Y. Arkun. Design of chemical plants with multiregime capabilities and robust dynamic operability characteristics. Computers and Chemical Engineering, 11(3):p205, 1987.

C.C. Pantelides. SPEEDUP - recent advances in process simulation. Computers and Chemical Engineering, 12:p745, 1988.

S. Papoulias and I.E. Grossmann. A structural optimization approach in process synthesis, parts I, II and III. Computers and Chemical Engineering, 7(6):p695, 1983.

J.D. Perkins and M.P.F. Wong. Assessing controllability of chemical plants. ChERD, 63:p358, 1985.

J.D. Perkins, C. Gannavarapu, and G.W. Barton. Choosing control structures based on economics. In Proceedings Control for Profit, Newcastle, 1989.

J.D. Perkins. Interactions between process design and process control. In $D Y$ CORD+ 1989 proceedings, Maastricht, The Netherlands, 1989. 
D.M. Prett and C.E. Garcia. Fundamental process control. Butterworths, Boston, 1988.

Prosys Technology. SPEEDUP case book. Prosys Technology, Cambridge, 1989.

D.E. Reeves, C.N. Nett, and Y. Arkun. Control configuration design for complex systems: A practical theory. Unpublished, Submitted to the IEEE Transactions on Automatic Control, 1991.

G.V. Reklaitis, A. Ravindran, and K.M. Ragsdell. Engineering optimization: methods and applications. Wiley-Interscience, 1983.

H.H. Rosenbrock. State space and multivariable theory. Nelson, London, 1970.

D.F. Rudd, G.J. Powers, and J.J. Siirola. Process synthesis. Prentice-Hall, Englewood Cliffs, 1973.

L.W. Russell and J.D. Perkins. Towards a method for diagnosis of controllability and operability problems in chemical plants. ChERD, 65:p453, 1987.

L.W. Russell. Control system synthesis for plants with time delays. PhD thesis, Dept. Chem. Eng., Imperial College, University of London, 1987.

R.W.H. Sargent and K. Gaminibandara. Optimal design of plate distillation columns. In Optimization in Action. Academic Press London, 1976.

R.W.H. Sargent and G.R. Sullivan. Development of an efficient optimal control package. In Proceedings of the 8th IFIP Conference on Cptimization Techniques, 1977.

R. Sarjeantson, editor. Metal bulletin's prices and data 1990. Metal Bulletin Books, Surrey, 1990.

N. Shah. Efficient scheduling, planning and design of multipurpose batch plants. PhD thesis, Dept. Chem. Eng., Imperial College, University of London, 1992.

R.K. Sinnot. Chemical Engineering, Volume 6, An Introduction to Chemical Engineering Design, Eds. J.M. Coulson and J.F. Richardson. Pergamon Press, Oxford, UK, 1983. 
S. Skogestad and M. Morari. Effect of disturbance directions on closed-loop performance. IEC Res., 26:p2029, 1987.

G. Stephanopoulos. Artificial intelligence and symbolic computing in process engineering design. In Proceedings of the third International Conference on Foundations of Computer Aided Process Design, page 21. Cache-Elsesvier, Amsterdam, 1990.

G. Stephanopoulos. Synthesis of control systems for chemical plants - a challenge for creativity. Computers and Chemical Engineering, 7(4):p331, 1983.

G. Stephanopoulos. Chemical process control. Prentice Hall, New Jersey, 1984.

J.M. Tyrrell. Process computer control - some myths explored. In I. Chem. E. Symposium Series No. 79, page p243. I. Chem. E. Publications, 1983.

T. Umeda, T. Kuriyama, and A. Ichikawa. A logical structure for process control system synthesis. In Proc. IFAC Congress Vol I, Helsinki, 1978.

S. Vasantharajan and L.T. Biegler. Simultaneous parameter optimization of differential-algebraic systems with enforcement of error criteria. Computers and Chemical Engineering, 14:1083, 1990.

J. Viswanathan and I.E. Grossmann. A combined penalty function and outer approximation method for MINLP optimization. Computers and Chemical Engineering, 14(7):769, 1990.

S.P. Walsh. Integrated design of chemical effluent treatment systems. PhD thesis, To be published, Dept. Chem. Eng., Imperial College, University of London, 1992.

A.W. Westerberg. A review of process synthesis. In ACS Symposium Series No. 124, page 53, 1980.

H. P. Williams. Model Building in Mathematical Programming. John Wiley and Sons, third edition, 1990.

M.P.F. Wong. Assessment of controllability of chemical processes. $\mathrm{PhD}$ thesis, Dept. Chem. Eng., Imperial College, University of London, 1984. 
A.M. Zakrzewski. Cost effective computer control - some examples. In I.Chem.E. Symposium Series No. 79, page p226. I. Chem. E. Publications, 1983.

J. Zhang, N.H. Kim, and L. Lasdon. An improved succesive linear programming algorithm. Management Science, 31(10):p1312, 1985. 


\section{Appendix A}

\section{Froth flotation circuit model}

This appendix summarises the process model and operating conditions for the froth flotation circuit case study in chapter 4 .

\section{A.1 Cell model}

To model the behaviour of the flotation cells, Barton et al. (1991) approximated the cell as a well mixed CSTR, with the flotation mechanism described by first order kinetics and an empirical factor affecting the kinetics to model air addition. The equations describing this cell model are given by Barton and Perkins (1988). For this model, three components were assumed:- water, valuables and gangue (Barton and Perkins further separated the solids into fast and slow floating). The cell model equations are summarised in the remainder of the section.

Component mass balances

$$
\begin{aligned}
& \frac{d M_{v}}{d t}=F_{v}-C_{v}-T_{v} \\
& \frac{d M_{g}}{d t}=F_{g}-C_{g}-T_{g}
\end{aligned}
$$

where $M_{x}, F_{x}, C_{x}$ and $T_{x}$ are respectively the mass of component $x$ in the cell, and the feed, concentrate and tailings flows of component $x$, where $x$ is the gangue $(g)$ or valuable $(v)$ component. A water component $(w)$ mass balance is not required, due to the assumption of constant cell volume (equation A.2). The model assumes that the tank is perfectly mixed, allowing calculation of the tailing's solids.

$$
\begin{aligned}
& T_{v}=T \frac{M_{v}}{M} \\
& T_{g}=T \frac{M_{g}}{M}
\end{aligned}
$$

$M$ is the total mass of the species in the cell. The total mass balance on tailings (A.1) ensures $T_{w}$ will obey the perfect mixing assumption.

Outlet stream total flows and cell mass total

$$
T=\sum_{i=v, g, w} T_{i}
$$




$$
\begin{aligned}
C & =\sum_{i=v, g, w} C_{i} \\
M & =\sum_{i=v, g, w} M_{i}
\end{aligned}
$$

$C$ and $T$ are the total concentrate and tailings flows. Flotation kinetics specify $C_{v}$ and $C_{g} . C_{w}$ is specified to be $46 \%$ of the total concentrate. The $k_{i}$ are first order rate constants and $a$ is an empirical air factor representing the aeration rate.

$$
\begin{aligned}
C_{v} & =k_{v} a M_{v} \\
C_{g} & =k_{g} a M_{g} \\
C_{w} & =0.46 \times C
\end{aligned}
$$

Perfect level control is assumed, leading to constant volume operation. The final equations specify the volume of the tank and ensure that the constant volume constraint on the tank is met, where $\rho_{i}$ is the density of component $i$ and $V$ is the volume of the cell.

$$
\begin{aligned}
V & =\frac{M_{v}}{\rho_{v}}+\frac{M_{g}}{\rho_{g}}+\frac{M_{w}}{\rho_{w}} \\
0 & =\frac{\left(F_{w}-C_{w}-T_{w}\right)}{\rho_{w}}+\frac{\left(F_{g}-C_{g}-T_{g}\right)}{\rho_{g}}+\frac{\left(F_{v}-C_{v}-T_{v}\right)}{\rho_{v}}
\end{aligned}
$$

\section{A.2 Operating conditions and constraints}

Tables A.1 and A.2 summarise the steady state operating conditions for the froth flotation circuit case study.

\begin{tabular}{|lc|c|}
\hline Feed water & $F_{w}^{0}$ & $1022 \mathrm{~kg} / \mathrm{min}$ \\
Feed gangue & $F_{g}^{0}$ & $1056 \mathrm{~kg} / \mathrm{min}$ \\
Feed valuable & $F_{v}^{0}$ & $144 \mathrm{~kg} / \mathrm{min}$ \\
\hline \multicolumn{3}{|c|}{ Steady state air factor $=1.0$} \\
\hline
\end{tabular}

Table A.1: Steady state operating conditions

\begin{tabular}{|l|r|r|r|}
\hline & Water & Gangue & Valuable \\
\hline Density $\left(\mathrm{kg} / \mathrm{m}^{3}\right)$ & 1000 & 3100 & 4000 \\
Rate constant $k_{\mathrm{i}}(\mathrm{hr}-1)$ & - & 0.8 & 12.0 \\
\hline
\end{tabular}

Table A.2: Physical properties 
For the design optimisation, the aeration rate and feed water flowrates were held at their nominal values, and the cell volumes were used as free variables. The optimisation maximised valuables recovery in the concentrate subject to the following design constraints:

Product grade $\geq 60 \%$

Total volume of cells $\leq 54 \mathrm{~m}^{3}$

For the operating optimisation, the cell volumes were fixed at their steady state optimum values, and the feed water and aeration rate were used as free variables. The operating valuables recovery was maximised subject to the following constraints:

Product grade $\geq 60 \%$

Feed water $\geq 46 \%$ of total feed slurry

Feed water $\leq 2044 \mathrm{~kg} / \mathrm{min}$ (maximum water flow is twice steady state flow)

Air factor $\geq 0.5$ (prevent air line flooding)

Air factor $\leq 2$ (prevent excessive slurry entrainment by violent agitation) 


\section{Appendix B}

\section{Scaling of condition numbers}

The lower the condition number of a control structure, the more likely it is to attain perfect control. Thus the process condition number may be used as measure of the attainability of perfectly controlled economics, and can be used in conjunction with the economics to choose a best control structure. The condition number of interest is that of the transfer function matrix describing the output variables in terms of the manipulated variables, i.e. $y(s)=G(s) u(s)$. Condition numbers of $G(j \omega)$ are then obtained for $\omega$, the frequency range of interest. This section summarises without proof some results on condition number scaling from Bauer (Bauer, 1963).

The condition number of a matrix $G$ subordinate to the p-norm is defined as:

$$
\operatorname{cond}_{p}(G)=\|G\|_{p} .\left\|G^{-1}\right\|_{p}
$$

where $p$ is 1,2 or $\infty$. The most commonly used condition number is the 2 -norm as it can be related to several measures of interest for process and control engineering (e.g. singular values). However the values of transfer function elements vary according to choice of units and variable ranges such as SI or imperial, percent or fraction, and the condition number changes accordingly. For comparison of different processes or processes at different conditions, it is desirable to have condition numbers which are independent of these choices. This may be achieved by pre- and post-multiplying the matrix by diagonal scaling matrices (a two sided scaling) and selecting the scaling matrices to minimise the condition number of the scaled matrix. Thus the minimum condition number of a matrix $G$ is defined as:

$$
\gamma_{p}^{*}=\min _{D_{1}, D_{2}} \operatorname{cond}_{p}\left(D_{1} G D_{2}\right)
$$

where $\gamma^{*}$ is the minimised condition number and the $D_{1}, D_{2}$ are the diagonal scaling matrices.

This problem has not been solved for the 2-norm, however Bauer (1963) gives a solution for the infinity norm and shows it to be an upper bound on the two 
norm condition number. In brief for an $n \times n$ matrix $A$ :

i) Obtain $A^{-1}$.

ii) Calculate the absolute values of the elements of $A$ and $A^{-1}\left(|A|\right.$ and $\left.\left|A^{-1}\right|\right)$.

iii) The $l_{\infty}$ minimum condition number is given by:

$$
\gamma_{\infty}^{*}=\max \text { eigenvalue }\left(|A| .\left|A^{-1}\right|\right)
$$

and

$$
\gamma_{\infty}^{*} \geq \gamma_{2}^{*}
$$

where $\gamma_{\infty}^{*}$ is an upper bound on the two norm minimum condition number $\gamma_{2}^{*}$. This value may then be used as a measure of the functional controllability as both a minimum condition number and a bound on the $l_{2}$ condition number.

\section{B.1 Minimum condition number of a triangular matrix}

For a triangular transfer function, the value of the upper bound of the $l_{2}$ minimised condition number is one (that is the $l_{\infty}$ minimum condition number). This can be proved by using the definition of the upper bound of the minimised condition number and the properties of triangular matrices. The inverse of a triangular matrix is also triangular, and the diagonal elements of the inverse matrix are the multiplicative inverses of the diagonal elements of the original matrix, then for a triangular matrix $A,|A| \cdot\left|A^{-1}\right|$ is also a triangular matrix with unity diagonals. The eigenvalues of a triangular matrix are the values of the diagonal elements, and therefore the maximum eigenvalue of $|A| \cdot\left|A^{-1}\right|$ is one. This is also the upper bound on the $l_{2}$ minimised condition number of the triangular matrix $A$ by the definition in the previous section. 


\section{Appendix C}

\section{Tight MILP formulation}

A tight control structure selection MILP formulation is summarised here for reference only. Although this representation is more efficient in terms of number of linear programs solved compared to the formulation in chapter 5 , the time required for problem solution is much higher for problems of realistic size. This is because the number of constraints in the LP increases proportionally to the number of disturbances in the problem, with a corresponding increase in solution time for each LP (the time for solution of an LP is proportional to between the square and cube of the number of constraints in the problem (Williams, 1990)).

The formulation consists of two stages: Development of a tight linear lower bound on the size of complex magnitude, and then use of this result to generate a tight linear approximation of the perfect control $\tilde{\sigma}$ (similar arguments apply to $\tilde{u}$ ).

\section{C.1 Improved linear lower bound on complex magnitudes}

Linear lower bounds on complex magnitudes are used in the calculation of $\tilde{s}$, the linear approximation to $\tilde{\sigma}$. The performance of the linear lower bound may be measured by the following relative error:

$$
e=100 \frac{r-r_{\min }}{r}
$$

Where $r$ is the length of the vector $a+i b\left(r^{2}=a^{2}+b^{2}\right)$, and $r_{\min }$ is the least linear estimate of $r$. For the bound developed in chapter 5:

$$
\begin{aligned}
& |r| \geq a \\
& |r| \geq-a \\
& |r| \geq b \\
& |r| \geq-b
\end{aligned}
$$

$r_{\min }$ is given by:

$$
r_{\min }=\min _{a, b} \max \{|a|,|b|\}
$$


Using geometrical arguments, this occurs at the intersection of the linear constraints $a=b$, when $r=\sqrt{2}|a|$ and $r_{\min }=|a|$, with a relative error of:

$$
\frac{\sqrt{2} a-a}{\sqrt{2} a} \approx 29.28 \%
$$

Consider the improved linear lower bound given by:

$$
\begin{aligned}
& |a| \geq a \\
& |a| \geq-a \\
& |b| \geq b \\
& |b| \geq-b \\
& r_{l} \geq|a| \\
& r_{l} \geq \frac{\sqrt{2+\sqrt{2}}}{2}|a|+\frac{\sqrt{2-\sqrt{2}}}{2}|b| \\
& r_{l} \geq \frac{|a|}{\sqrt{2}+\frac{|b|}{\sqrt{2}}} \\
& r_{l} \geq \frac{\sqrt{2-\sqrt{2}}}{2}|a|+\frac{\sqrt{2+\sqrt{2}}}{2}|b| \\
& r_{l} \geq|b|
\end{aligned}
$$

where $r_{l}$ is a linear lower bound on $|r|$, with a maximum relative error of $e \approx 2 \%$.

\section{C.1.1 Proof of bounding properties}

The bounding properties of the above formulation have already been demonstrated with the exception of equations C.2 to C.4. The bounding property may be proved by showing that when these bounds are treated as equalities (i.e. active bounds), their maximum values underestimate $|r|$. The maximum value of the lower bound may be obtained by making the appropriate equation an equality, substituting $b=\sqrt{r^{2}-a^{2}}$, solving $\frac{d r_{l}}{d a}=0$ for $a^{*}$ and hence $r_{l}^{\max }$. This will be demonstrated for constraint C.3. Considering the vector $a+i b$ in the first quadrant, setting C.3 to an equality and substituting to obtain:

$$
r_{l}=\frac{a+\sqrt{r^{2}-a^{2}}}{\sqrt{2}}
$$

Solving $\frac{d r_{l}}{d a}=0$ for the maximum value of $r_{l}$ yields:

$$
r_{l}^{\max }=r \text { at } a=b=\frac{r}{\sqrt{2}}
$$


Similarly for C.2:

$$
\begin{aligned}
r_{l}^{\max }=r \quad \text { at } \quad a & =\frac{\sqrt{2+\sqrt{2}}}{2} r \\
b & =\frac{\sqrt{2-\sqrt{2}}}{2} r
\end{aligned}
$$

and for C.3 (as expected by symmetry):

$$
\begin{aligned}
r_{l}^{\max }=r \quad \text { at } \quad a & =\frac{\sqrt{2-\sqrt{2}}}{2} r \\
b & =\frac{\sqrt{2+\sqrt{2}}}{2} r
\end{aligned}
$$

In all cases, $r_{l}^{\max }$ equals $|r|$ (i.e. the maximum active value of the linear lower bound on $|r|$ is in fact $|r|)$. Therefore equations C.2-C.4 are valid lower bounds on $r$.

\section{C.1.2 Maximum relative error}

The maximum relative error will occur at the intersection of a pair of active bounds (the bounds are defined so that no more than two bounds can be active at any one time). The method of calculation is to define $r_{l 1}$ and $r_{l 2}$ to be equalities, set $b=\sqrt{r^{2}-a^{2}}$ and set $r_{l 1}=r_{l 2}$, solve for $a, b$ and hence $r_{\min }$ and then calculate $e$ for each intersection, selecting the maximum relative error as the worst of these values. The table C.1 lists the values of $e$ at the intersections of the active constraints, showing a maximum relative error of $e \approx 2 \%$ :

\begin{tabular}{|c|c|c|c|c|c|}
\hline Equation $r_{l 1}$ & Equation $r_{l 2}$ & $a$ & $b$ & $r_{\min }$ & $e \approx(\%)$ \\
\hline C. 1 & C.2 & $0.98078 r$ & $0.19509 r$ & $0.98078 r$ & $2 \%$ \\
C.2 & C.3 & $0.83147 r$ & $0.55557 r$ & $0.98078 r$ & $2 \%$ \\
C.3 & C.4 & $0.55557 r$ & $0.83147 r$ & $0.98078 r$ & $2 \%$ \\
C.4 & C.5 & $0.19509 r$ & $0.98078 r$ & $0.98078 r$ & $2 \%$ \\
\hline
\end{tabular}

Table C.1: Maximum relative error for improved complex magnitude bound

\section{C.2 Improved lower bound for $\tilde{s}$}

In the MILP representation developed in chapter 5 , it is noted that for a given control structure, the relationship between the estimated maximum constraint 
back off vector $\tilde{\sigma}$ and the disturbance vector $p(\omega)$ is nonlinear:

$$
\tilde{\sigma}=|G(j \omega)| p(\omega)
$$

where $G(j \omega)$ is the transfer function between $\bar{\sigma}$ and $\bar{p}$. To maintain the linear properties of the problem, it was noted that a lower bound on $\tilde{\sigma}$ is given by:

$$
\tilde{\sigma} \geq \tilde{s}=|G(j \omega) p(\omega)|
$$

where $G(j \omega) p(\omega)$ is obtained in the course of the solution of the MILP, and linear lower bounds on the complex magnitude have been developed (chapter 5 and the previous section of this appendix). To obtain a more accurate estimate of $\tilde{\sigma}$, it would be desirable to develop a linear approximation to $|G(j \omega)|$. The remainder of this section addresses this problem.

Consider the form of $\tilde{\sigma}$ :

$$
\tilde{\sigma}_{i}=\max _{\Phi}|\sigma|=\sum_{i=1}^{n_{d}}\left|g_{i j}\right| p(\omega)_{j}
$$

where $g_{i j}$ is the transfer function element between disturbance $j$ and slack variable $i$ under the usual perfect control assumptions. Using the previously developed ideas, it is possible to obtain an accurate linear approximation of the magnitude of a complex number. Therefore if the transfer function elements $g_{i j}$ can be calculated during the LP, a good approximation to $\tilde{\sigma}$ can be obtained from:

$$
\tilde{\sigma}_{i} \approx \sum_{i=1}^{n_{d}} \hat{g}_{i j} p(\omega)_{j}
$$

where $\hat{g}_{i j}$ is the linear approximation to $\left|g_{i j}\right|$.

Applying these results to the MILP problem in section 5.2.3 leads to the following modifications: 
1. Replace the equations involving $x_{r}, x_{c}$, etc. with the following system:

$$
\left.\begin{array}{rl}
-E_{j} & =A \bar{x}_{r, j}+B \bar{u}_{r, j}+C \bar{z}_{r, j}+\omega I_{x} \bar{x}_{c, j} \\
-L_{j} & =F \bar{x}_{r, j}+G \bar{u}_{r, j}+H \bar{z}_{r, j} \\
-R_{j} & =M \bar{x}_{r, j}+N \bar{u}_{r, j}+O \bar{z}_{r, j}+I_{\sigma} g_{r, j} \\
0 & =-\omega I_{x} \bar{x}_{r, j}+A \bar{x}_{c, j}+B \bar{u}_{c, j}+C \bar{z}_{c, j} \\
0 & =F \bar{x}_{c, j}+G \bar{u}_{c, j}+H \bar{z}_{c, j} \\
0 & =M \bar{x}_{c, j}+N \bar{u}_{c, j}+O \bar{z}_{c, j}+I_{\sigma} g_{c, j} \\
\bar{z}^{h} & \geq \mathcal{X}^{T} I_{z} \bar{z}^{h}+\bar{z}_{r, j} \\
\bar{z}^{h} & \geq \mathcal{X}^{T} I_{z} \bar{z}^{h}+\bar{z}_{c, j} \\
\bar{z}^{h} & \geq \mathcal{X}^{T} I_{z} \bar{z}^{h}-\bar{z}_{r, j} \\
\bar{z}^{h} & \geq \mathcal{X}^{T} I_{z} \bar{z}^{h}-\bar{z}_{c, j} \\
X^{T} I_{u} \bar{u}^{h} & \geq \bar{u}_{r, j} \\
X^{T} I_{u} \bar{u}^{h} & \geq \bar{u}_{c, j} \\
X^{T} I_{u} \bar{u}^{h} & \geq-\bar{u}_{r, j} \\
X^{T} I_{u} \bar{u}^{h} & \geq-\bar{u}_{c, j}
\end{array}\right\} \quad \forall j=1,2, \ldots, n_{p}
$$

This corresponds to solving the frequency response problem for each disturbance $\bar{p}_{j}(\omega)=1+j 0$, where $E_{j}, L_{j}$ and $R_{j}$ are the columns of the matrices corresponding to the $j$ th disturbance. $x_{r, j}, x_{c, j}$, etc. are the vectors of responses of the process to the unit disturbance. In particular, $g_{r, j}$ and $g_{c, j}$ are the real and complex vectors corresponding to $j$ th column of the transfer function $G(j \omega)$ between $\bar{\sigma}$ and $\bar{p}$.

2. Apply the linear bounds procedure in the previous section to obtain a tight lower bound, $\hat{g}_{i j}$, on the magnitude of the elements of the transfer function $G(j \omega)$.

3. Calculate a tight linear approximation of $\tilde{\sigma}$ as:

$$
\tilde{\rho}_{i}=\sum_{j=1}^{n_{p}} \hat{g}_{i j} p(\omega)_{j} \quad \forall i=1,2, \ldots, n_{\sigma}
$$

A similar approach is applied to obtain a tight lower bound on $U$, the maximum dynamic variation in the manipulated variables. 


\section{Appendix D}

\section{Double effect evaporator models and process items}

The nonlinear dynamic model of the double effect evaporator is based on the model given in the SPEEDUP Casebook (Prosys Technology, 1989), but the evaporator model has been rewritten to take vapour holdup into account, and a more detailed description of the physical parameters of the evaporator units has also been developed.

\section{D.1 Pumps}

All pumps use the characteristic equation used in the SPEEDUP Casebook (Prosys Technology, 1989) double effect evaporator example.

If $\left(P_{o}-P_{i}\right)>\Delta P_{\max }$ then

$$
F=F_{\max }\left(\frac{\Delta P_{\min }}{\Delta P_{\max }}\right)^{0.25}
$$

Else if $\left(P_{o}-P_{i}\right)<\Delta P_{\min }$ then

$$
F=F_{\max }
$$

Else

$$
F=F_{\max }\left(\frac{\Delta P_{\max }-\left(P_{o}-P_{i}\right)+\Delta P_{\min }}{\Delta P_{\max }}\right)^{0.25}
$$

$F$ is the mass flow through the pump.

$P_{i}$ is the inlet pressure.

$P_{o}$ is the outlet pressure.

$\Delta P_{\max }$ is the maximum operating pressure difference.

$\Delta P_{\min }$ is the minimum operating pressure difference.

$F_{\max }$ is the maximum operating flow.

The operating parameters for the pumps are given in table D.1. 


\begin{tabular}{|l|r|r|r|}
\hline Pump & $\Delta P_{\max } \mathrm{kPa}$ & $\Delta P_{\min } \mathrm{kPa}$ & $F_{\max } \mathrm{kg} / \mathrm{min}$ \\
\hline Feed & 200.0 & 10.0 & 135.0 \\
Intermediate & 160.0 & 10.0 & 100.0 \\
Product & 200.0 & 10.0 & 60.0 \\
\hline
\end{tabular}

Table D.1: Evaporator pump characteristics

\section{D.2 Valves}

All valves were modelled as square root pressure drop valves of the form:

$$
F^{2}=K_{v} \Delta P
$$

$F$ is the mass flow.

$K_{v}$ is the square of the valve coefficient.

$\Delta P$ is the pressure drop across the valve.

\section{D.3 Evaporator}

\section{D.3.1 Evaporator design}

Each evaporator effect consists of two hemispherical ends and a cylindrical section (figure D.1) with diameter $1.62 \mathrm{~m}$ and height $10.5 \mathrm{~m}$. The steam chest is $9 \mathrm{~m}$ high, starting at the base of the cylindrical section, leaving a $0.7 \mathrm{~m}$ freeboard to the top of the cylinder for liquor in the effect. The steam chest consists of a 22 by 22 bundle of 1 inch tubes on a square pitch, with separation of 2 inches centre to centre. This yields a surface area for heat exchange of $340 \mathrm{~m}^{2}$. The total volume of the liquor side is $5.81 \mathrm{~m}^{3}$, and the steam chest is covered at $3.25 \mathrm{~m}^{3}$. The maximum volume the liquor may occupy (to the top of the freeboard) is $4.7 \mathrm{~m}^{3}$. When the steam chest is covered, the static head at the liquor inlet is $9 \mathrm{~m}$ and the static head at the outlet is $9.8 \mathrm{~m}$, plus the head of liquor in the freeboard.

\section{D.3.2 Evaporator model}

The original model in the SPEEDUP Casebook did not take the static head of liquor or the vapour holdup in the evaporator into account. The model has 


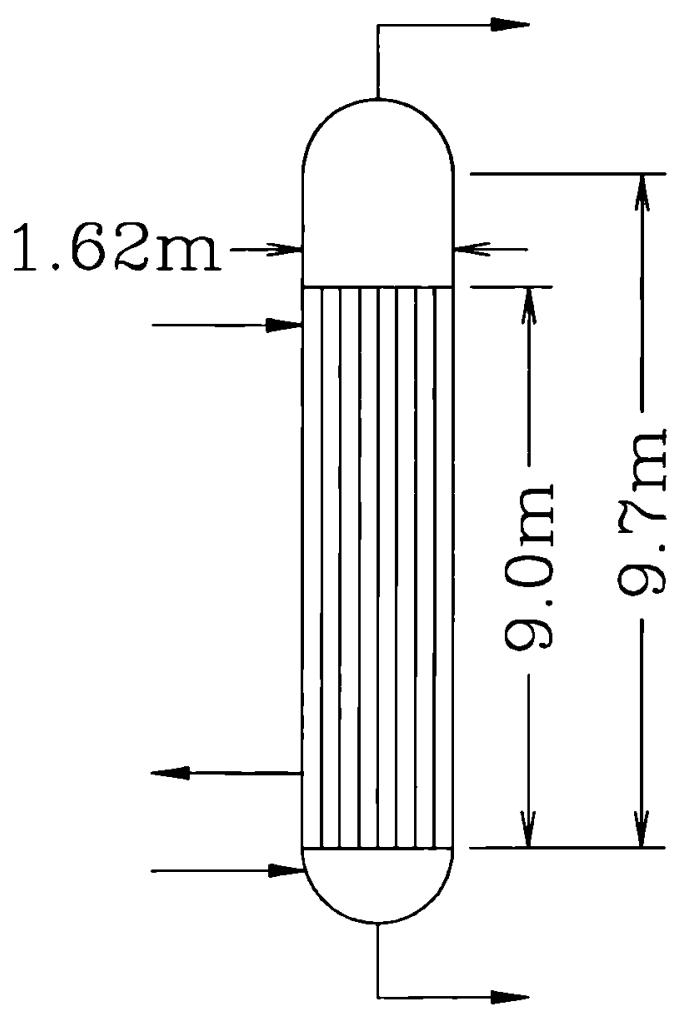

Figure D.1: Evaporator geometry

been rewritten to handle these effects and the above modifications to the physical description of the process.

Assumptions for the evaporator model:

1. Thermal equilibrium between gas and liquid phases.

2. The water vapour behaves as a perfect gas.

3. The solution obeys Raoult's Law.

4. The liquor is incompressible.

5. The solute has a negligible vapour pressure.

6. Constant heat of vaporisation and specific heats.

7. The liquor is perfectly mixed.

The symbols for the evaporator model are defined in table D.2. 
Mass balances

$$
\begin{gathered}
\frac{d H_{w}}{d t}=F_{l i}-F_{l o}-F_{v o} \\
\frac{d H_{s}}{d t}=F_{s i}-F_{s o}
\end{gathered}
$$

Volume relations

$$
\begin{gathered}
H_{w}=H_{l}+H_{v} \\
V=V_{l}+V_{v} \\
V_{v} \rho_{v}=H_{v} \\
V_{l}=\frac{H_{l}}{\rho_{l}}+\frac{H_{s}}{\rho_{s}} \\
V_{l} \rho_{a}=H_{l}+H_{s}
\end{gathered}
$$

Energy relations

$$
\begin{gathered}
\frac{d I}{d t}=E_{l i}-E_{l o}-E_{v o}+Q \\
E_{l o}=\left(F_{l o} C_{p w}+F_{s o} C_{p s}\right)\left(T-T_{r}\right) \\
E_{v o}=F_{v o}\left(\lambda+C_{p w}\right)\left(T-T_{r}\right) \\
I=\left(H_{l} C_{p w}+H_{s} C_{p s}\right)\left(T-T_{r}\right) \\
Q=S_{i} \lambda \\
Q=U A\left(T_{c}-T\right)
\end{gathered}
$$

Perfect mixing

$$
F_{s o}\left(H_{l}+H_{s}\right)=H_{s}\left(F_{l o}+F s o\right)
$$

Vapour pressure from Antoine equation and Raoult's Law, vapour density from perfect gas equation.

$$
\begin{gathered}
P=\frac{\frac{H_{L}}{M_{w}} P^{0}}{\frac{H_{1}}{M_{w}}+\frac{H_{s}}{M_{s}}} \\
P^{0}=A \times 10^{\left(B-\frac{c}{T+D}\right)} \\
\rho_{v}=\frac{P M_{w}}{R(T+273)}
\end{gathered}
$$

Total head at liquor entry and exit points

$$
P_{i}=P+(9.0+h) g \rho_{a}
$$




$$
\begin{gathered}
P_{o}=P+(9.8+h) g \rho_{a} \\
h=\frac{4\left(V_{l}-3.25\right)}{\pi 1.62^{2}}
\end{gathered}
$$

Steam chest pressure from Antoine equation

$$
P^{c}=A \times 10^{\left(B-\frac{C}{T_{c}+D}\right)}
$$

The values of the process parameters are given in table D.3.

The feed pressure of the utility steam is given by the Antoine equation used for the steam chest pressure.

\section{D.4 Condition number plots}

The condition number plots for disturbance cases 2 and 3 are included here for reference use with the evaporator MILP case study. 
$F_{l i} \quad$ mass flow of water in the feed liquor

$F_{l 0} \quad$ mass flow of water in the product liquor

$F_{v o} \quad$ mass flow of vapour from the effect

$F_{s i} \quad$ mass flow of solute in the feed liquor

$F_{\text {so }} \quad$ mass flow of solute in the product liquor

$H_{w} \quad$ holdup of water

$H_{s} \quad$ holdup of solute

$H_{l} \quad$ holdup of water as liquor

$H_{v} \quad$ holdup of water as vapour

$V \quad$ volume of process side

$V_{l} \quad$ volume of liquor

$V_{v} \quad$ volume of vapour

$\rho_{v} \quad$ vapour density

$\rho_{l} \quad$ density of liquid water

$\rho_{s} \quad$ density of solute

$\rho_{a} \quad$ liquor density

$I \quad$ internal energy

$E_{l i} \quad$ enthalpy of feed liquor

$E_{l o} \quad$ enthalpy of product liquor

$E_{v o} \quad$ enthalpy of product vapour

$Q \quad$ heat transfer

$C_{p w} \quad$ specific heat of liquid water

$C_{p s} \quad$ specific heat of solute

$T \quad$ temperature

$T_{r} \quad$ reference temperature

$\lambda$ steam heat of vaporisation

$S_{i} \quad$ steam feed flowrate

$U \quad$ heat transfer coefficient

$A_{h} \quad$ heat transfer area

$M_{w} \quad$ relative molar mass of water

$M_{s} \quad$ relative molar mass of solute

$P \quad$ pressure

$A, B, C, D \quad$ Antoine coefficients

$T \quad$ temperature

$R \quad$ gas coefficient

$h \quad$ liquor head in the freeboard

$P_{\text {o }} \quad$ liquor outlet pressure

$P_{i} \quad$ liquor inlet pressure

Table D.2: Symbol definitions for evaporator model 


\begin{tabular}{|l|l|}
\hline$V$ & $5.81 \mathrm{~m}^{3}$ \\
$\rho_{w}$ & $1000 \mathrm{~kg} / \mathrm{m}^{3}$ \\
$\rho_{s}$ & $1114 \mathrm{~kg} / \mathrm{m}^{3}$ \\
$C_{p w}$ & $4.183 \mathrm{~kJ} / \mathrm{kg} / \mathrm{K}$ \\
$C_{p s}$ & $2.4 \mathrm{~kJ} / \mathrm{kg} / \mathrm{K}$ \\
$M_{w}$ & $18.02 \mathrm{kgmol} / \mathrm{kg}$ \\
$M_{s}$ & $62.0 \mathrm{kgmol} / \mathrm{kg}$ \\
$T_{r}$ & $60 \mathrm{C}$ \\
$A_{h}$ & $340 \mathrm{~m}^{2}$ \\
$U$ effect 1 & $20 \mathrm{~kJ} / \mathrm{m}^{2}$ \\
$U$ effect 2 & $30 \mathrm{~kJ} / \mathrm{m}^{2}$ \\
$A$ & 0.1333 \\
$B$ & 7.96681 \\
$C$ & 1668.21 \\
$D$ & 228.0 \\
\hline
\end{tabular}

Table D.3: Evaporator parameters 


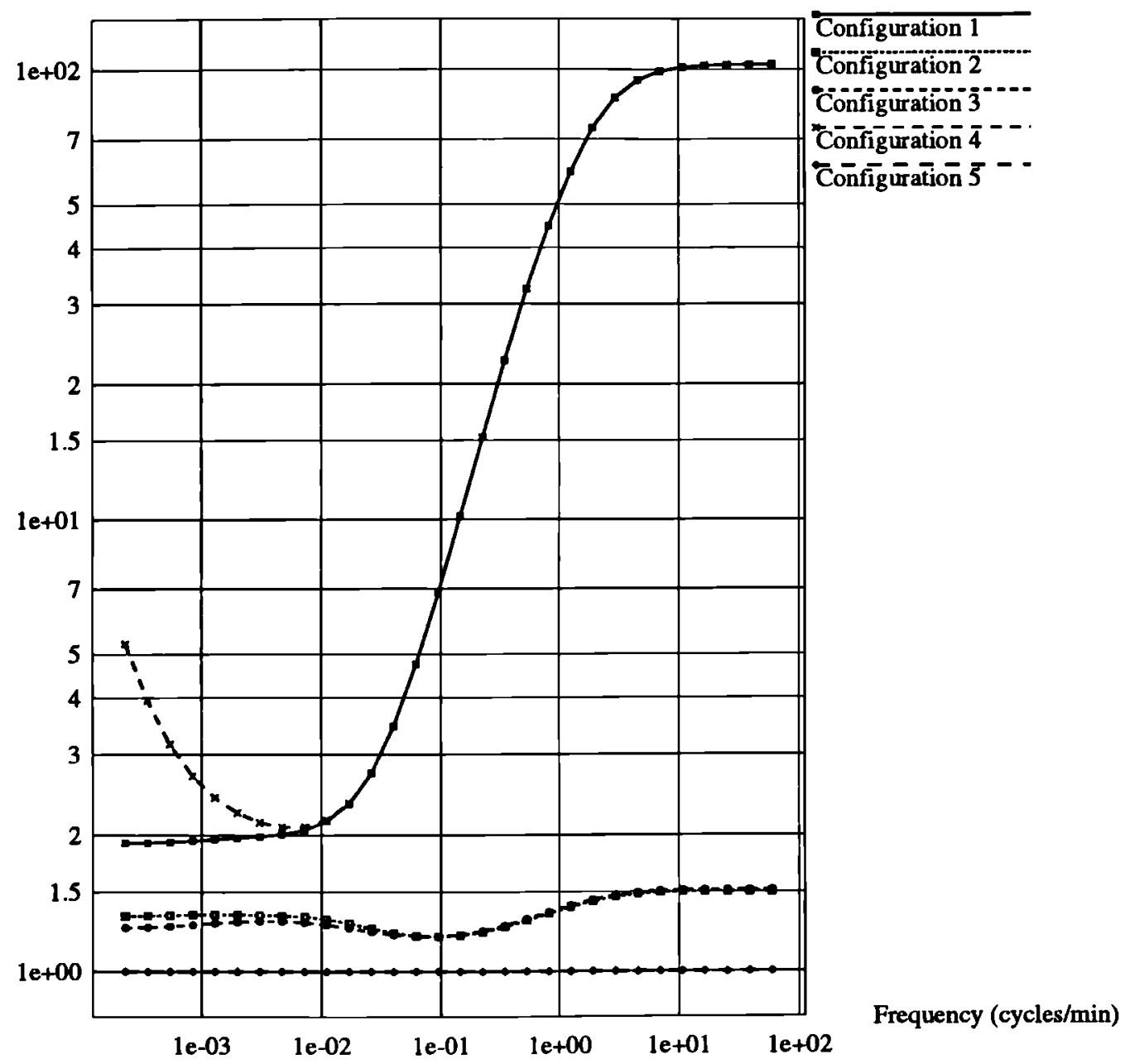

Figure D.2: $l_{\infty}$ minimum condition number plot for case 2 


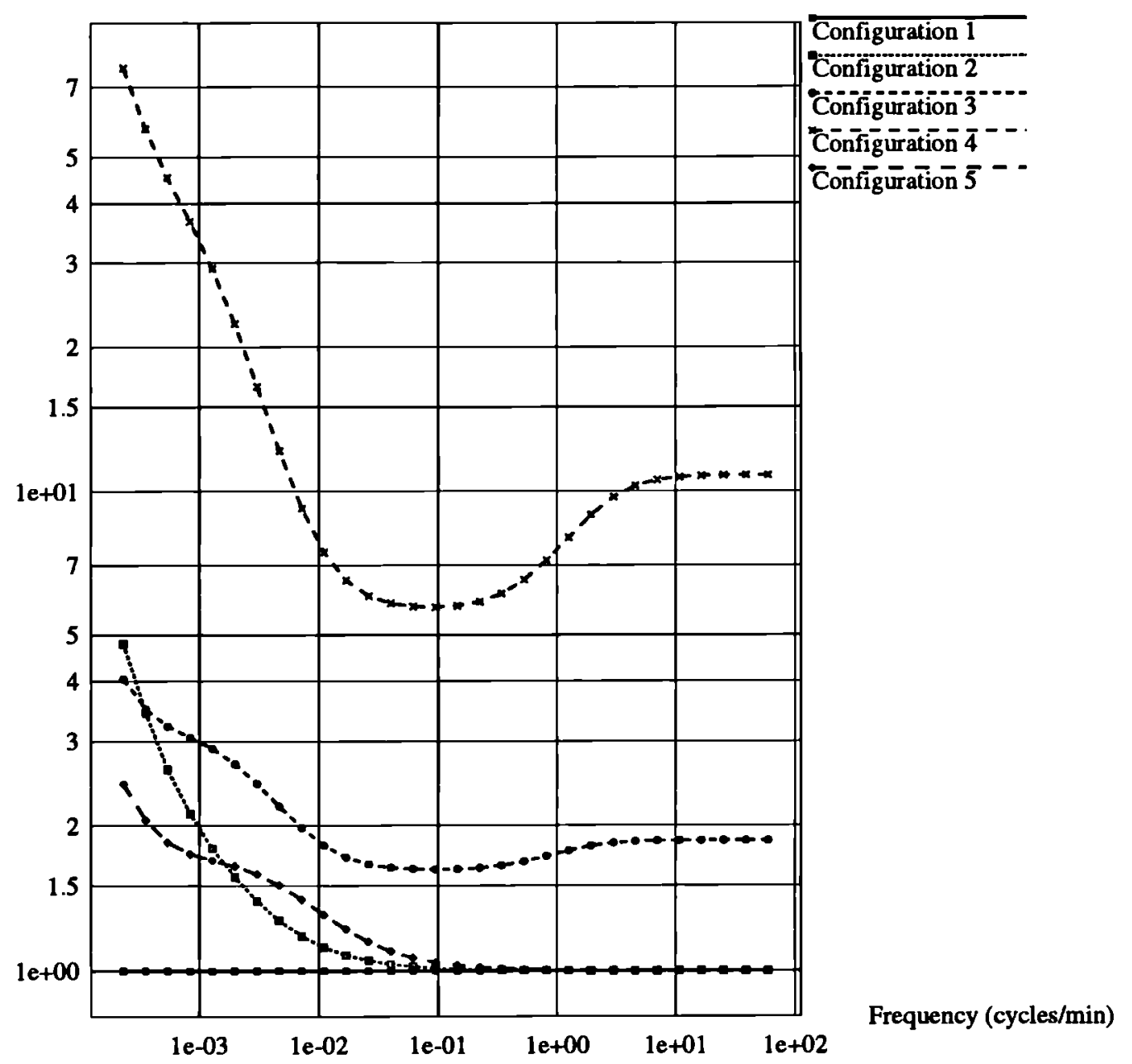

Figure D.3: $l_{\infty}$ minimum condition number plot for multiple frequency case 


\section{Appendix E}

\section{Mount Isa Mines model changes}

The models and process constants used are identical to those used by Perkins and Barton (1988), except for minor modifications to the flotation cell model and the collector addition model, which are detailed here. The only major difference is that the controllers are omitted for the MILP analysis. A process grade is the mass percentage of copper in the solids, and a process recovery is the mass percentage of copper recovered from the feed copper.

\section{E.1 Flotation cell model}

The cell model required the addition of a copper balance for use in the objective function. The copper is only present in the two valuable components (Fewings et al., 1979), and is assumed to be in the same proportion for both valuable components. It is assumed that the mineral holdup in the froth phase is negligible, and therefore the copper assay in the concentrate and the tailings is equal. This leads to a copper balance of the form:

$$
\begin{gathered}
\frac{d M_{C u}}{d t}=\left(F_{v s}+F_{v f}\right) A_{i}-\left(T_{v s}+T_{v f}+C_{v s}+C_{v f}\right) A_{o} \\
M_{C u}=M\left(X_{v s}+X_{v f}\right) A_{o}
\end{gathered}
$$

$M_{C u}$ mass of copper in the cell

$v s, v f$ slow floating and fast floating valuable components

$F, T, C$ feed, tailings and concentrate mass flows

$A_{i}, A_{o}$ copper assay (mass fraction) in feed and product valuables

$M$ mass of material in the cell

$X$ mass fraction of component in the cell

For all remaining units, the copper balance is a simple steady state equation. 


\section{E.2 Collector addition model}

The collector model was altered so that the collector addition modified, rather than defined, the proportion of fast floating valuables. This is a more appropriate approach as it limits the effectiveness of the collector (as would be seen in practice). It was assumed that the collector could only improve the fast floating valuables by a maximum of $20 \%$ of the combined feed flow of the valuables. The modified equations are of the form:

$$
\begin{gathered}
O_{v f}=I_{v f}+\left(I_{v f}+I_{v s}\right) u_{c} \\
O_{v f}+O_{v s}=I_{v f}+I_{v s}
\end{gathered}
$$

$I, O$ inlet and outlet mass flows of the collector addition unit

$u_{c}$ collector effect (with an upper bound of $20 \%$ )

\section{E.3 Summary of steady state operating conditions}

The optimum steady state variable values are summarised here for reference.

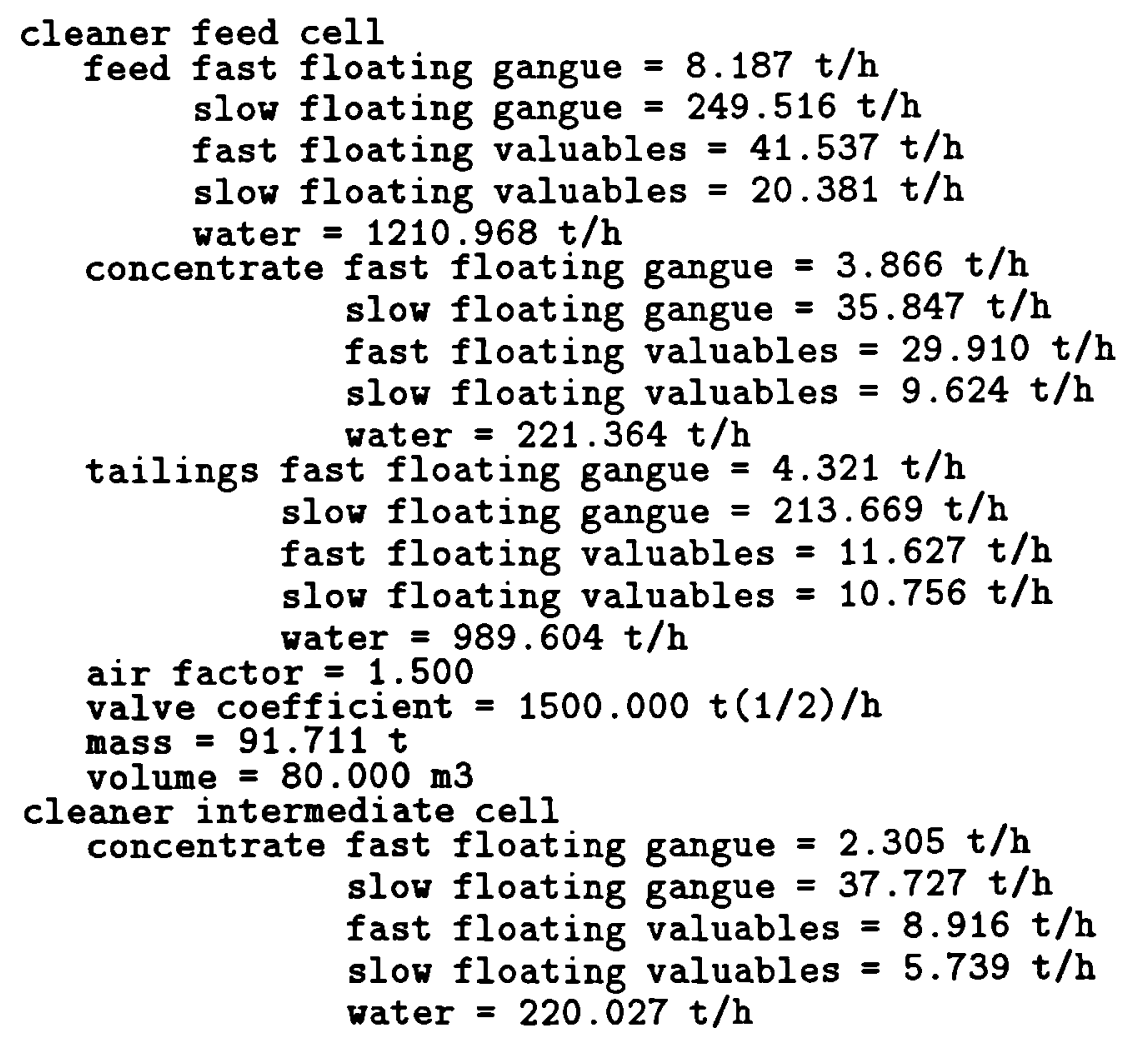




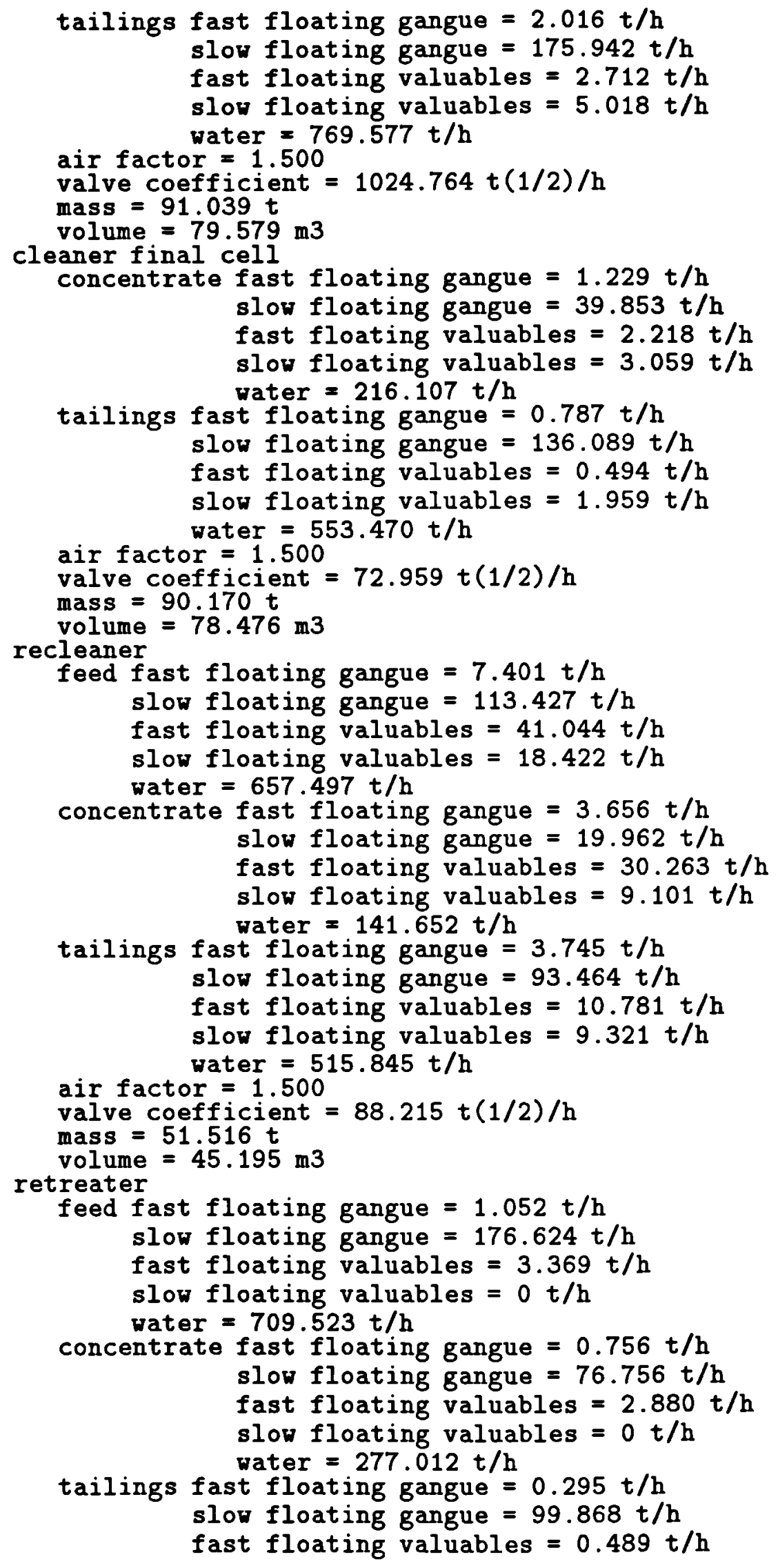




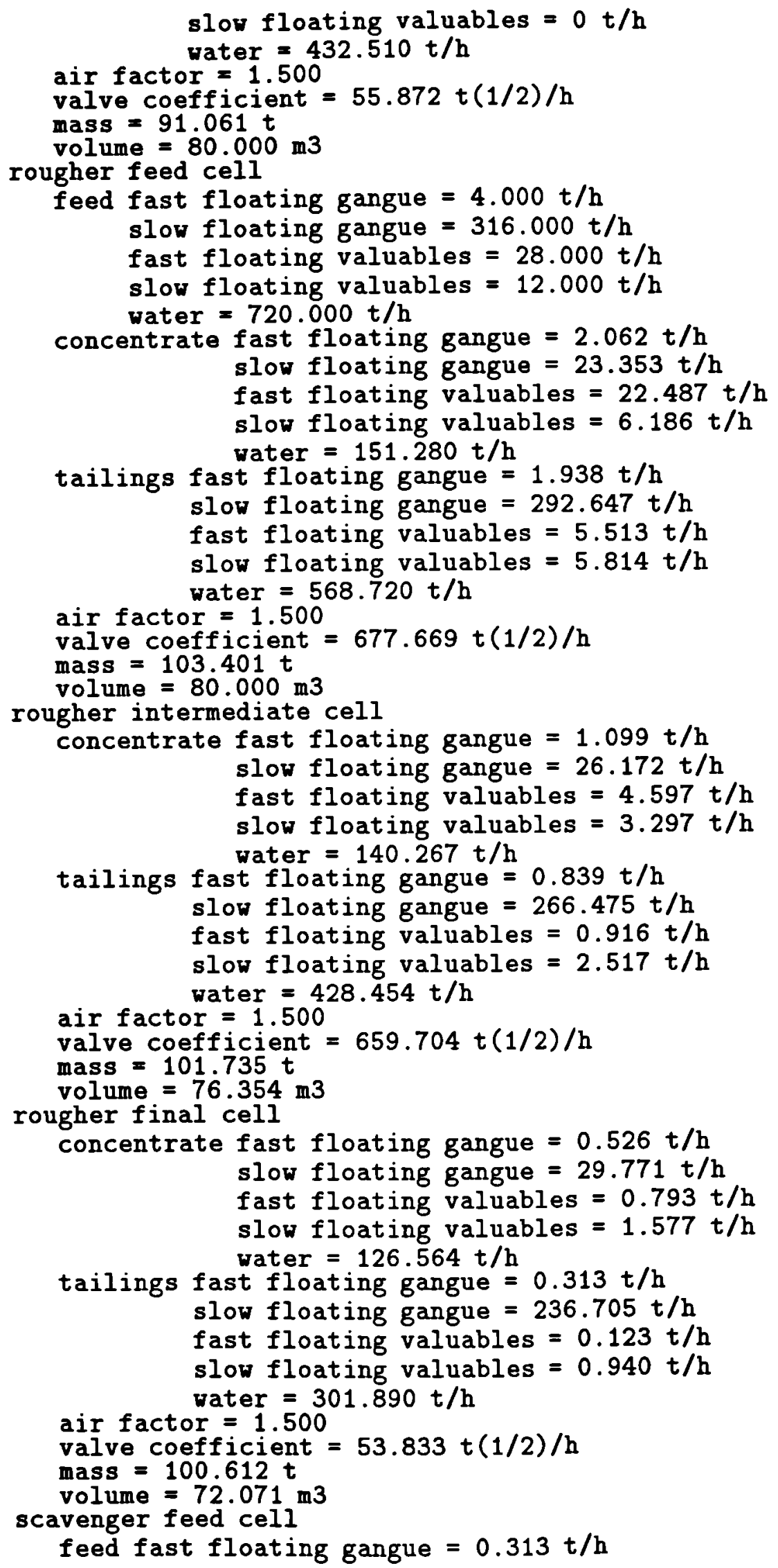




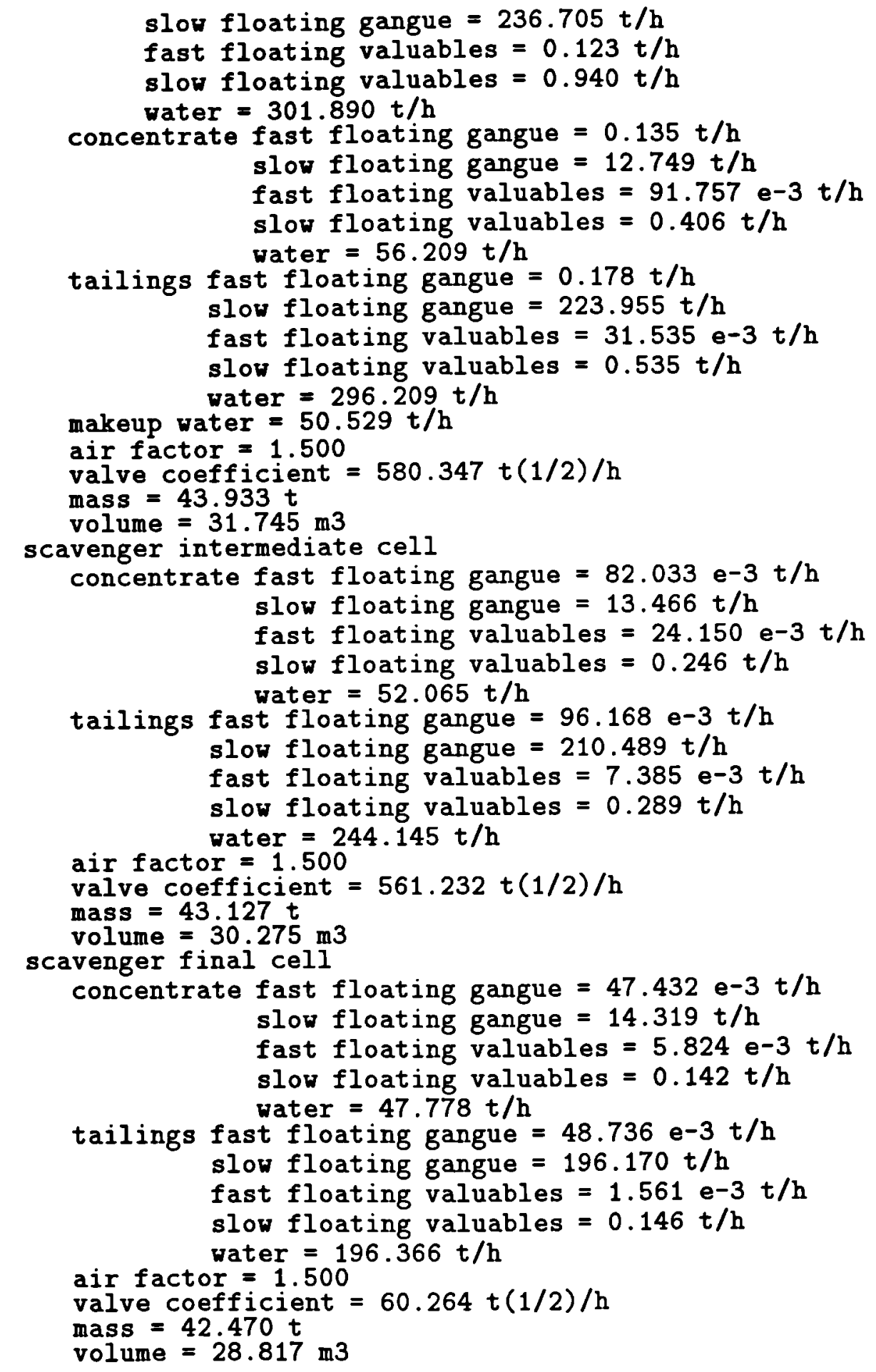




\section{Appendix F}

\section{Properties of $\gamma_{\infty}^{*}$ for square block triangular transfer functions}

This appendix contains a proof of the theorem that the $l_{\infty}$ minimum condition number of a square block triangular transfer function is given by the maximum $l_{\infty}$ minimum condition number of the square subsystems.

Theorem Given a square block triangular transfer function matrix $O$ of the form:

$$
O=\left[\begin{array}{ccccc}
A & 0 & \cdots & \cdots & 0 \\
X & B & & & \vdots \\
X & X & C & & \vdots \\
\vdots & & & \ddots & \vdots \\
X & \cdots & \cdots & \cdots & N
\end{array}\right]
$$

where $A, B, C, \ldots, N$ are square matrices, then the $l_{\infty}$ norm minimum condition number, $\gamma_{\infty}^{*}$, of $O$ is given by:

$$
\gamma_{\infty}^{*}(O)=\max \left\{\gamma_{\infty}^{*}(A), \gamma_{\infty}^{*}(B), \gamma_{\infty}^{*}(C), \ldots, \gamma_{\infty}^{*}(N)\right\}
$$

\section{Proof}

If the above theorem holds for the matrix:

$$
P=\left[\begin{array}{ll}
A & 0 \\
Q & B
\end{array}\right]
$$

then it will hold for an arbitrarily large square block triangular system, as the system may be decomposed into a series of subsystems of the form $P$.

The $l_{\infty}$ norm minimum condition number of $P$ is given by:

$$
\gamma_{\infty}^{*}(P)=\max \lambda\left(|P| \cdot\left|P^{-1}\right|\right)
$$

where $\lambda$ is an eigenvalue. The inverse of the matrix $P$ is:

$$
P^{-1}=\left[\begin{array}{cc}
A^{-1} & 0 \\
-B^{-1} Q A^{-1} & B^{-1}
\end{array}\right]
$$


yielding

$$
\gamma_{\infty}^{*}(P)=\max \lambda\left(\left[\begin{array}{cc}
|A| .\left|A^{-1}\right| & 0 \\
|Q| .\left|A^{-1}\right|+|B| .\left|B^{-1} Q A^{-1}\right| & |B| .\left|B^{-1}\right|
\end{array}\right]\right)
$$

Using the standard definition of eigenvalues for a matrix $R$ :

$$
\operatorname{det}(\lambda I-R)=0
$$

and expanding cofactors yields:

$$
\operatorname{det}\left(\lambda I_{A}-|A| .\left|A^{-1}\right|\right) \operatorname{det}\left(\lambda I_{B}-|B| .\left|B^{-1}\right|\right)=0
$$

and noting that:

$$
\begin{aligned}
& \gamma_{\infty}^{*}(A)=\max \lambda\left(|A| \cdot\left|A^{-1}\right|\right) \\
& \gamma_{\infty}^{*}(B)=\max \lambda\left(|B| \cdot\left|B^{-1}\right|\right)
\end{aligned}
$$

It may then be concluded:

$$
\gamma_{\infty}^{*}(P)=\max \left\{\gamma_{\infty}^{*}(A), \gamma_{\infty}^{*}(B)\right\}
$$

The theorem has some general application, as is can be applied to large systems to determine which subsystem is limiting the controllability, and then effort can be concentrated on improving the subsystem controllability, rather than attempting to improve the controllability on a plant wide basis. 


\section{Appendix G}

\section{Path constraints in dynamic optimisation}

This appendix contains two brief notes describing scaling and perturbation of path constraints in the form of terminal equality constraints for improved optimiser performance.

\section{G.1 Scaling of path constraints}

Consider the constraint $c^{\prime} \geq 0$. The SQP code employed in this thesis will terminate and treat the constraint as active if:

$$
-t_{e} \leq c^{\prime} \leq 0
$$

where $t_{e}$ is the optimisation tolerance. To improve the performance of the numerical integration, it is desirable to scale the path constraints within the model to have a value of -1 at the maximum acceptable constraint violation, and reformulate the path constraints as (Walsh, 1992):

$$
c t_{e} \geq 0
$$

The full description of a path constraint $c$ is given by:

$$
\begin{aligned}
c & =y\left(t=t_{f}\right) \\
\dot{y} & =-\max (g(x), 0)^{2} \\
y(t=0) & =0
\end{aligned}
$$

where it is required that

$$
g(x) \leq 0 \quad \forall t \in\left[0, t_{f}\right]
$$

The constraint violation of $c$ corresponds to the integral of the squares of the violations of $g$ over time $t=0$ to $t=t_{f}$ :

$$
\text { violation } c=\int_{0}^{t_{f}}(\text { violation } g(x))^{2} d t
$$


If an upper bound on the acceptable violation of $g$ can be posed, then this can be used to scale the path constraint such that at the maximum acceptable constraint violation $c=-1$. The next step is to develop a method for calculating the maximum acceptable constraint violation. This may be done by considering the maximum possible violation for each constraint as a rectangular pulse (Walsh, 1992). Therefore for each constraint a peak violation of the constraint, $\Delta v$, and the maximum time the peak violation may be sustained, $\Delta t$, must be specified. Examining this rectangular pulse with equation $\mathrm{G} .1$ yields:

$$
\begin{aligned}
\max \text { violation } & =\int_{0}^{t_{f}}(\max (g(x), 0))^{2} d t \\
& =\int_{0}^{t_{f}}(\Delta v)^{2} d t \\
& =(\Delta v)^{2} \Delta t
\end{aligned}
$$

For example, given a path constraint on a process flow which is of the order of $10000 \mathrm{~m}^{3} / \mathrm{s}$, the peak violation of the constraint may be chosen to be a violation of $1 \mathrm{~m}^{3} / \mathrm{s}$, and if the integration is being carried out over a $100 \mathrm{~s}$, then $\Delta t$ would typically be specified to be 0.1 to $1 \mathrm{~s}$, yielding a maximum acceptable violation of 0.01 to $1 \mathrm{~m}^{6} / \mathrm{s}$. By choosing the acceptable violations, we are in effect specifying the required tolerance on each constraint. Once the maximum integral square violation has been specified, the scaled path constraint may be written as:

$$
\begin{aligned}
c t_{e} & \geq 0 \\
c & =y\left(t=t_{f}\right) \\
(\Delta v)^{2} \Delta t \dot{y} & =-\max (g(x), 0)^{2} \\
y(t=0) & =0
\end{aligned}
$$

Which scales $c$ to a value of -1 at the maximum acceptable constraint violation.

\section{G.2 Perturbed problem solution}

Using the above formulation, the path constraints are in the form:

$$
c t_{e} \geq 0
$$


where an active constraint lies in the range:

$$
-1 \leq c \leq 0
$$

However the convergence properties of this constraint are poor as $c \rightarrow 0$. The convergence properties can be greatly improved without a significant loss in solution accuracy by using the following perturbed path constraint:

$$
(2 c+1) t_{e} \geq 0
$$

where an active constraint now lies in the range:

$$
-1 \leq c \leq-\frac{1}{2}
$$

Using this perturbed constraint can reduce the number of iterations required to locate the optimum by up to a factor of two (Walsh, 1992), and therefore all path constraints have been used in this form. 